\title{
Photochemical Chemoselective Alkylation of Tryptophan-containing Peptides
}

\author{
Benjamin Laroche, ${ }^{\ddagger a}$ Xinjun Tang, ${ }^{\ddagger a}$ Gaétan Archer, ${ }^{\text {a }}$ Riccardo Di Sanza,${ }^{a}$ and Paolo \\ Melchiorre*,a,b \\ ${ }^{a} I C I Q$ - Institute of Chemical Research of Catalonia, the Barcelona Institute of Science and Technology, \\ Avinguda Països Catalans 16, 43007 Tarragona, Spain \\ ' ICREA - Passeig Lluís Companys 23, 08010 Barcelona, Spain \\ ${ }^{\dagger}$ B. Laroche and X. Tang contributed equally \\ *Correspondence to: pmelchiorre@iciq.es
}




\section{Table of Contents}

A. General Information page S3

B. Optimization studies with 2,4-dinitrobenzylbromide 2a page S4

B.1 Screening of solvents $\quad$ page S4

B.2 Screening of base and equivalents of acceptor page S5

B.3 Screening of light sources $\quad$ page S6

B.4 Reaction procedure and characterization of product 3a page S6

C. Optimization studies with the Katritzky salt 4a page S7

C.1 Identification and characterization of the of EDA complex $\quad$ page S7

$\begin{array}{ll}\text { C.2 Screening of the light sources } & \text { page S8 }\end{array}$

C.3 Screening of bases page S9

C.4 Screening of water-soluble solvents $\quad$ page S10

C.5 Investigations of other radical precursors $\quad$ page S11

D. Syntheses of dipeptide, tripeptide and tetrapeptide starting materials (1a-1o) page S11

D.1 General procedure for amide coupling page S11

D.2 Characterization data of compounds 1a-1o page S11

E. Syntheses of pyridinium salts $4 \quad$ page S16

E.1 General procedure page S16

E.2 Characterization data of compounds $\mathbf{4 b - 4 g} \quad$ page S17

F. Photochemical alkylation of Trp-containing peptides $\quad$ page S20

F.1 General procedure page S20

F.2 Picture of the reaction setup $\quad$ page $S 20$

F.3 Characterization data of compounds 5a-5p and 6a-6j $\quad$ page S20

G. 1 mmol scale reaction $\quad$ page S32

H. Unsuccessful substrates $\quad$ page S33

I. Possible chain mechanism $\quad$ page S34

J. References $\quad$ page S35

$\begin{array}{lr}\text { K. NMR spectra } & \text { page S36 }\end{array}$ 


\section{A. General Information}

The NMR spectra were recorded at $300 \mathrm{MHz}, 400 \mathrm{MHz}$, and $500 \mathrm{MHz}$ for ${ }^{1} \mathrm{H}$ or at $100 \mathrm{MHz}$ and $125 \mathrm{MHz}$ for ${ }^{13} \mathrm{C}$, respectively. The chemical shifts $(\delta)$ for ${ }^{1} \mathrm{H}$ and ${ }^{13} \mathrm{C}$ are given in ppm relative to residual signals of the solvents $\left(\mathrm{CHCl}_{3} @ 7.26 \mathrm{ppm}{ }^{1} \mathrm{H} \mathrm{NMR}, 77.36 \mathrm{ppm}{ }^{13} \mathrm{C} \mathrm{NMR}\right)$. Coupling constants are given in $\mathrm{Hz}$. The following abbreviations are used to indicate the multiplicity: $\mathrm{s}$, singlet; d, doublet; $\mathrm{t}$, triplet; q, quartet; $\mathrm{m}$, multiplet; br s, broad signal.

High-resolution mass spectra (HRMS) were obtained from the ICIQ High Resolution Mass Spectrometry Unit on MicroTOF Focus and Maxis Impact (Bruker Daltonics) with electrospray ionization.

UV-vis measurements were carried out on a Shimadzu UV-2401PC spectrophotometer equipped with photomultiplier detector, double beam optics and D2 and W light sources.

General Procedures. All reactions were set up under an argon atmosphere in oven-dried glassware or glass vial using standard Schlenk techniques, unless otherwise stated. Synthesis grade solvents were used as purchased. Anhydrous solvents were taken from a commercial SPS solvent dispenser. Chromatographic purification of products was accomplished using force-flow chromatography (FC) on silica gel (35-70 mesh). For thin layer chromatography (TLC) analysis throughout this work, Merck precoated TLC plates (silica gel 60 GF254, $0.25 \mathrm{~mm}$ ) were used, using UV light as the visualizing agent and either phosphomolybdic acid in EtOH, dinitrophenylhydrazine in $\mathrm{EtOH} / \mathrm{H}_{2} \mathrm{O}$, Vanilin acidic solution in $\mathrm{EtOH}$ or basic aqueous potassium permangante $\left(\mathrm{KMnO}_{4}\right)$, and heat as developing agents. Organic solutions were concentrated under reduced pressure on a Büchi rotary evaporator (in vacuo at $40^{\circ} \mathrm{C}, \sim 5 \mathrm{mbar}$ ).

Materials: Commercial grade reagents and solvents were purchased at the highest commercial quality from Sigma Aldrich, Fluka, Acros Organics, Fluorochem or Alfa Aesar and used as received, unless otherwise stated. Peptides (1) and Katritzky salts (4) were prepared according to known literature procedures; details are provided in other sections of this manuscript.

Photochemistry: All photochemical reactions were carried out using a Kessil lamp PR160L (https://www.kessil.com/science/PR160L.php) with wavelength of peak intensity of $390 \mathrm{~nm}$, lamp intensity set to $100 \%$ and positioned at a distance of $4 \mathrm{~cm}$ from the reaction vessel, unless otherwise stated. 
B. Optimization studies with 2,4-dinitrobenzylbromide 2a

B.1 Screening of solvents

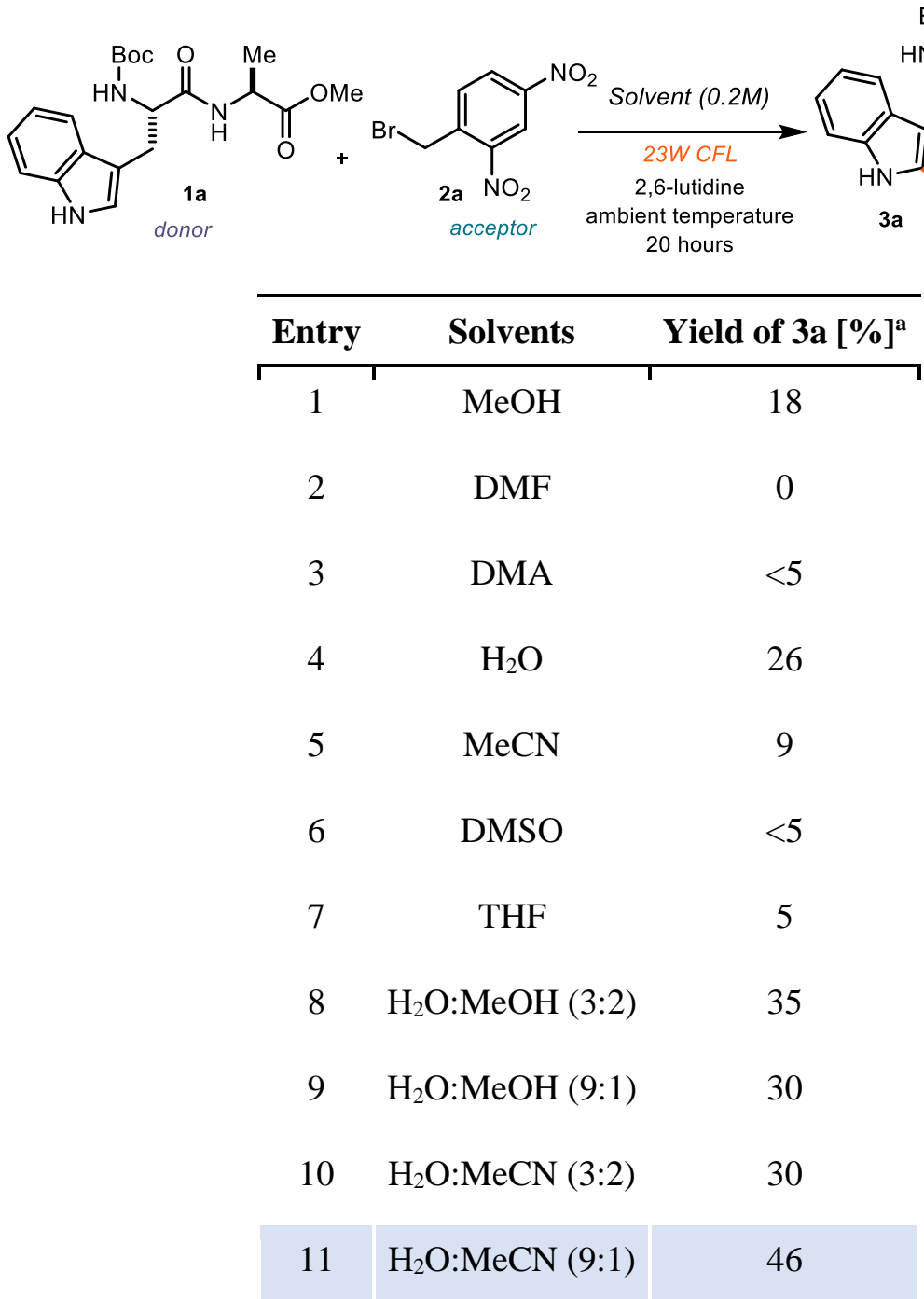

a Yields of 3a determined by ${ }^{1} \mathrm{H}$ NMR analysis of the crude mixture using 1,2,3-trimethoxybenzene as the internal standard. 


\section{B.2 Screening of base and equivalents of acceptor}

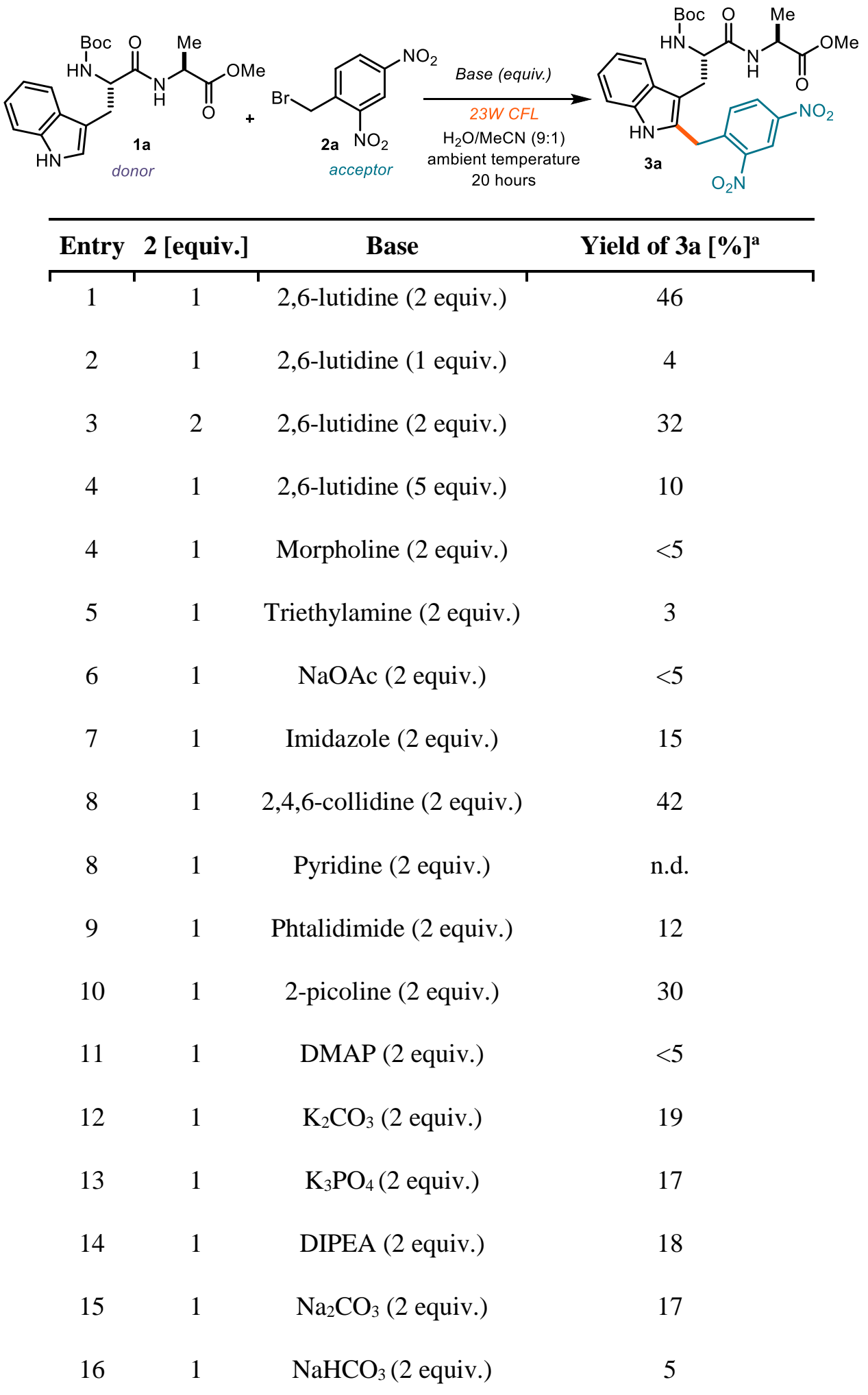

a Yields of 3a determined by ${ }^{1} \mathrm{H}$ NMR analysis of the crude mixture using 1,2,3-trimethoxybenzene as the internal standard. 


\section{B.3 Screening of light sources}

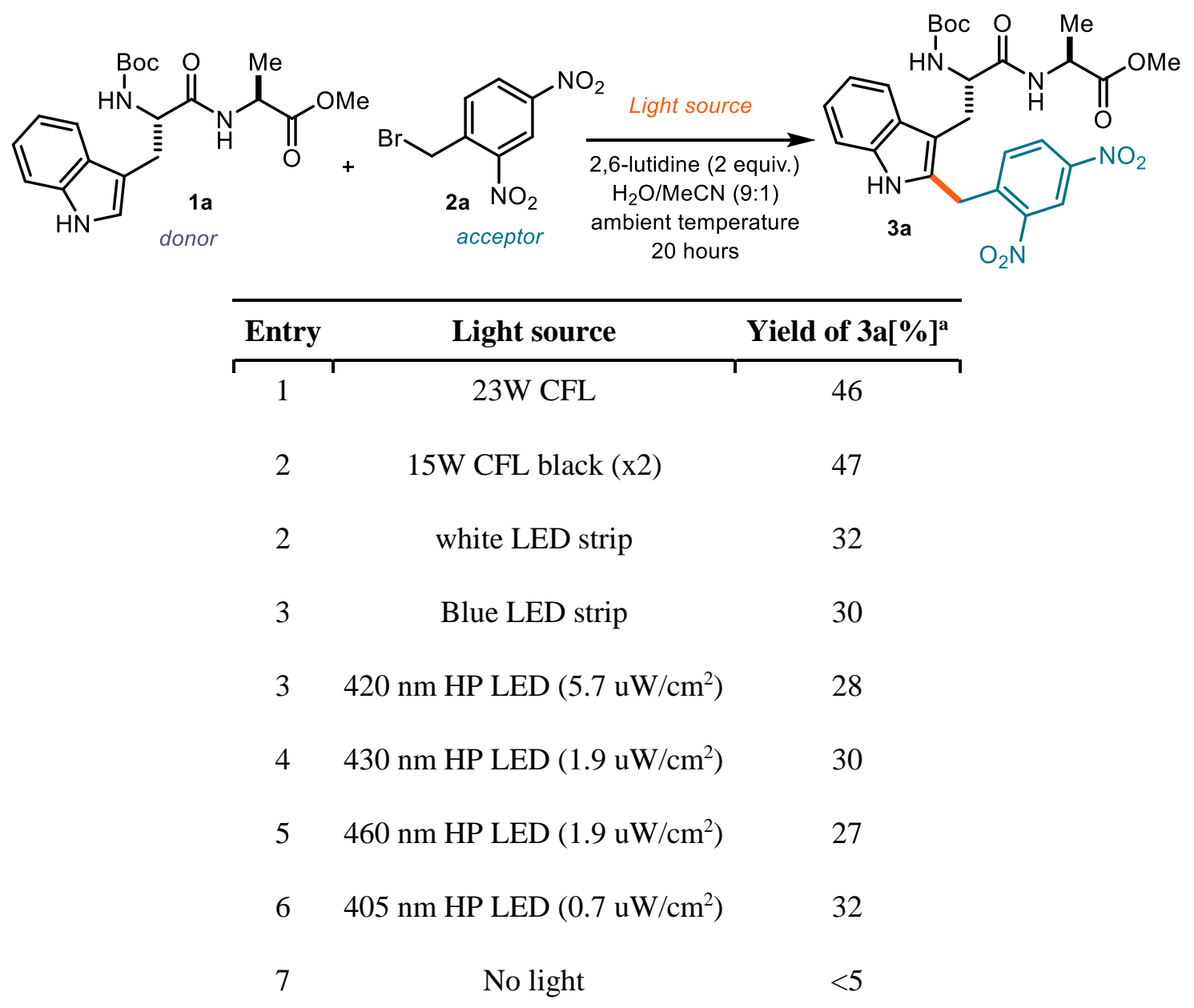

a Yields of 3a determined by ${ }^{1} \mathrm{H}$ NMR analysis of the crude mixture using 1,2,3-trimethoxybenzene as the internal standard.

\section{B.4 Reaction procedure and characterization of product $3 a$}

Dipeptide 1a (39 mg, $0.1 \mathrm{mmol})$ and dinitrobenzylbromide $\mathbf{2 a}(26 \mathrm{mg}, 0.1 \mathrm{mmol})$ were charged into a Schlenk tube. Then the solvent $\left(\mathrm{H}_{2} \mathrm{O}: \mathrm{MeCN} 9: 1,0.2 \mathrm{M}\right)$ and 2,6-ludidine $(23 \mu \mathrm{L}, 0.2 \mathrm{mmol})$ were sequentially added. The mixture was treated with a freeze-pump-thaw cycle (3 times) to remove oxygen. The resulting degassed solution was irradiated with $23 \mathrm{~W}$ CFL white light bulb positioned at approximatively $1-\mathrm{cm}$ distance from the reaction tube for 20 hours at ambient temperature. The resulting mixture was evaporated under reduced pressure and purified by chromatography on silica gel (hexane/EtOAc, gradient from 3:1 to 3:2) to afford compound 3a as an orange solid ( $26.4 \mathrm{mg}, 46 \%$ yield).<smiles>CC(=O)C(NC(=O)[C@@H](Cc1c[nH]c2ccccc12)NC(=O)c1ccccc1)C(=O)O</smiles>

${ }^{1} \mathrm{H} \mathrm{NMR}\left(500 \mathrm{MHz}, \mathrm{CDCl}_{3}\right): \delta 8.80(\mathrm{~d}, J=2.4 \mathrm{~Hz}, 1 \mathrm{H}), 8.31(\mathrm{dd}, J=$ $8.6,2.4 \mathrm{~Hz}, 1 \mathrm{H}), 8.21(\mathrm{~s}, 1 \mathrm{H}), 7.59(\mathrm{~d}, J=7.9 \mathrm{~Hz}, 1 \mathrm{H}), 7.55(\mathrm{~d}, J=$ $8.6 \mathrm{~Hz}, 1 \mathrm{H}), 7.27(\mathrm{~s}, 1 \mathrm{H}), 7.19-7.14(\mathrm{~m}, 1 \mathrm{H}), 7.13-7.07(\mathrm{~m}, 1 \mathrm{H})$, $6.17(\mathrm{~d}, J=7.1 \mathrm{~Hz}, 1 \mathrm{H}), 5.28(\mathrm{~s}, 1 \mathrm{H}), 4.64-4.44(\mathrm{~m}, 2 \mathrm{H}), 4.43-4.25$ $(\mathrm{m}, 2 \mathrm{H}), 3.60(\mathrm{~s}, 3 \mathrm{H}), 3.25(\mathrm{dd}, J=64.5,11.8 \mathrm{~Hz}, 2 \mathrm{H}), 1.42(\mathrm{~s}, 9 \mathrm{H})$, $1.27(\mathrm{~d}, J=7.1 \mathrm{~Hz}, 3 \mathrm{H})$. 
${ }^{13} \mathrm{C}$ NMR $\left(126 \mathrm{MHz}, \mathrm{CDCl}_{3}\right) \delta 172.7,171.0,155.4,149.0,146.9,141.0,136.0,133.7,131.6$, 128.3, 127.6, 122.7, 120.5, 120.3, 118.8, 111.1, 109.1, 80.4, 52.6, 48.4, 29.4, 28.4, 28.0, 18.6. HRMS (ESI) Exact mass calculated for $\mathrm{C}_{27} \mathrm{H}_{31} \mathrm{~N}_{5} \mathrm{NaO}_{9}[\mathrm{M}+\mathrm{H}]^{+}:$592.2014; found: 592.1993.

\section{Optimization reactions for Katritzky salt as acceptor}

\section{C.1 Identification of the EDA complex}

UV-Vis absorption experiments were performed using the model substrates 1a and 4a. As depicted in Figure S1, the dipeptide 1a absorbs between 300 to $380 \mathrm{~nm}$, whereas the Katritzky pyridinium salt 4a slightly absorbs in the visible region (till about $420 \mathrm{~nm}$, orange line in the Figure S1). Upon mixing the substrates $1 \mathbf{a}$ and $\mathbf{4 a}$, we observed a visibly yellow color solution (left side, Figure S1). The mixture of $\mathbf{1 a}$ and $\mathbf{4 a}$ displayed a significant bathochromic shift in absorbance (to about $440 \mathrm{~nm}$, grey line).

Interestingly, the combination of $\mathbf{1 a}, \mathbf{4 a}$, and $\mathrm{NaOAc}$ led to a further red-shift in the absorption (yellow line), which may suggest that the acetate anion might facilitate the formation of an EDA complex. It is however noteworthy that the model reaction proceeds also in the absence of NaOAc, indicating that the EDA complex formed upon aggregation of substrates 1a and $\mathbf{4 a}$ is responsible for the photochemical radical process to occur (see entry 4 in Table 1 of the main text).
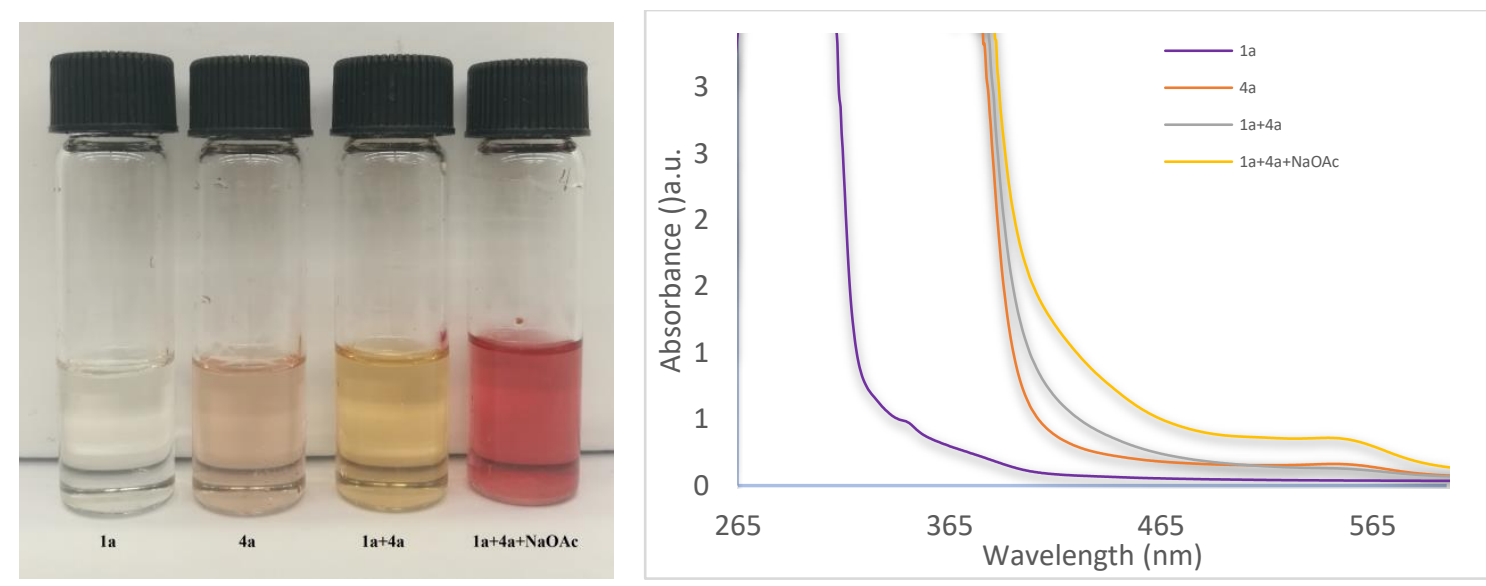

Figure S1. Absorption spectra of 1a, 4a, 1a+4a, 1a+4a+NaOAc $(c=0.1 \mathrm{mM}$ in DMA $)$ 


\section{C.2 Screening of the light sources}

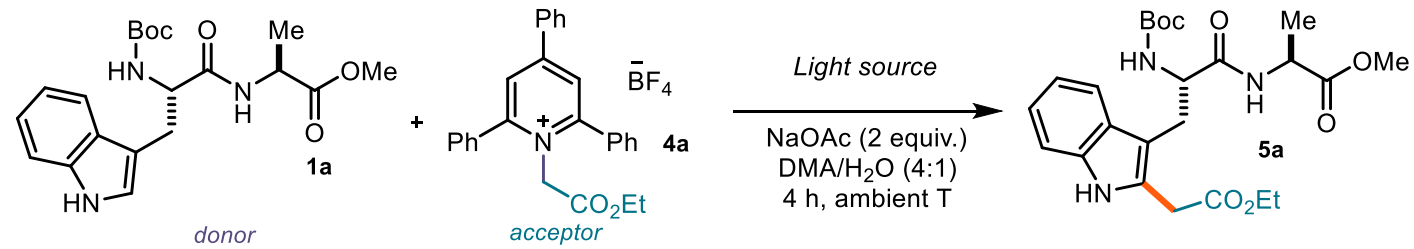

\begin{tabular}{|c|c|c|}
\hline Entry & Light source & Yield of 5a [\% ] \\
\hline 1 & Kessil (390 nm, 100\%) & $75(68)$ \\
\hline 2 & Kessil (427 nm, 100\%) & 11 \\
\hline 3 & Kessil (456 nm, 100\%) & 17 \\
\hline 4 & Kessil (390 nm, 75\%) & 48 \\
\hline 5 & - & 0 \\
\hline
\end{tabular}

a Yields of 5a determined by ${ }^{1} \mathrm{H}$ NMR analysis of the crude mixture using 1,2,3-trimethoxybenzene as the internal standard; yields of isolated $\mathbf{5 a}$ are reported in brackets. 


\section{C.3 Screening of bases}

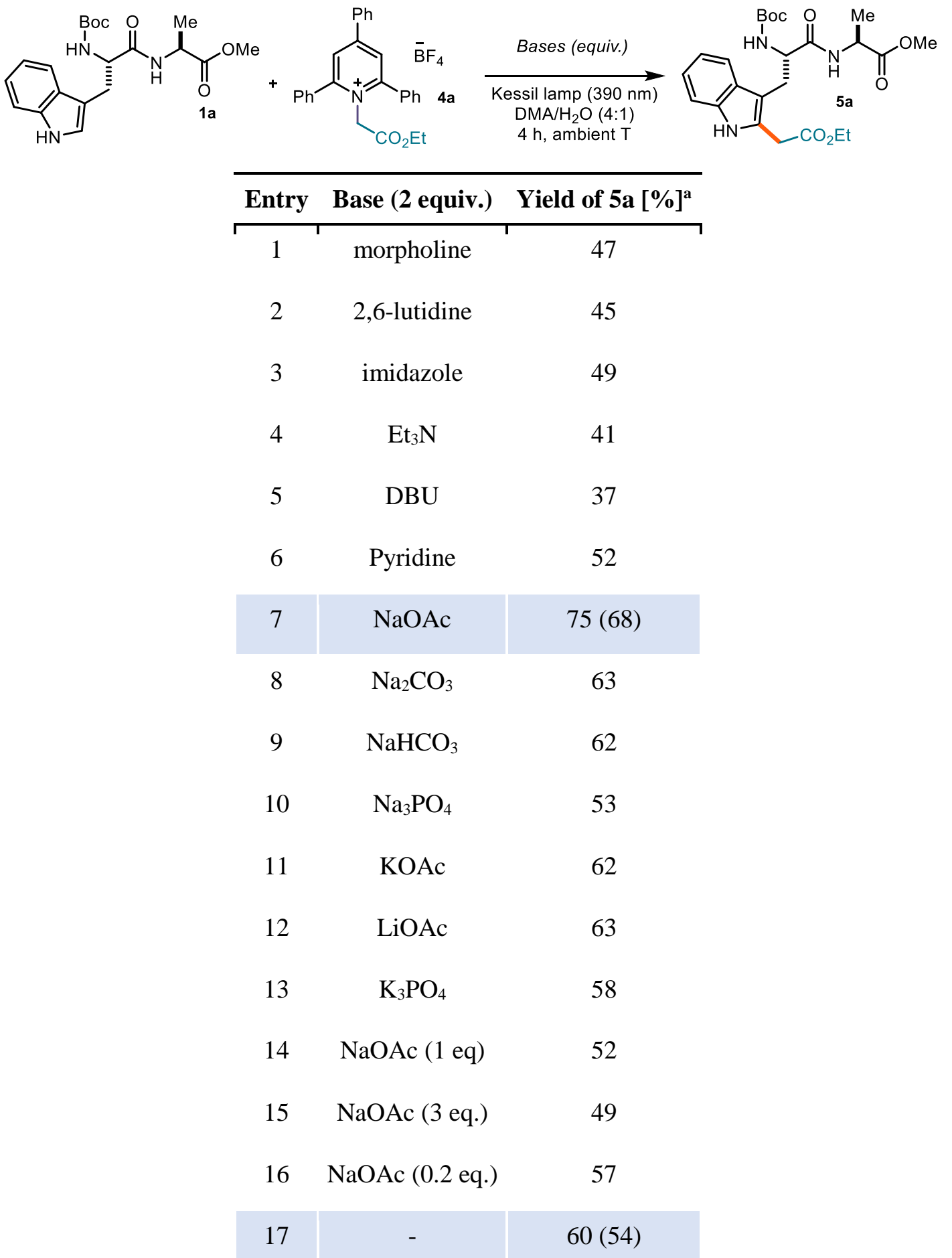

${ }^{\mathbf{a}}$ Yields of $\mathbf{5 a}$ determined by ${ }^{1} \mathrm{H}$ NMR analysis of the crude mixture using 1,2,3-trimethoxybenzene as the internal standard; yields of isolated $\mathbf{5} \mathbf{a}$ are reported in brackets 


\section{C.4 Screening of water-soluble solvents}

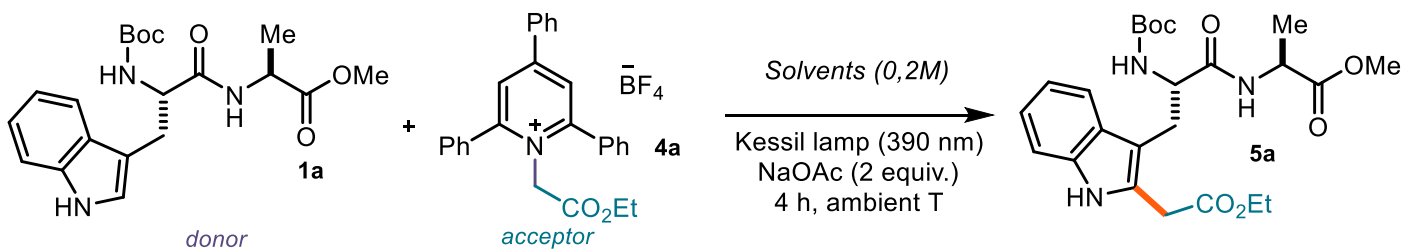

\begin{tabular}{|c|c|c|}
\hline Entry & Solvent $(0,2 M)$ & Yield of $5 \mathrm{a}[\%]^{\mathrm{a}}$ \\
\hline 1 & DMA: $\mathrm{H}_{2} \mathrm{O}(4: 1)$ & $75(68)$ \\
\hline 2 & $\mathrm{H}_{2} \mathrm{O}$ & $6^{\mathrm{b}}$ \\
\hline 3 & $\mathrm{DMF}: \mathrm{H}_{2} \mathrm{O}(4: 1)$ & 70 \\
\hline 4 & DMSO: $\mathrm{H}_{2} \mathrm{O}(4: 1)$ & 59 \\
\hline 5 & $\mathrm{EtOH}: \mathrm{H}_{2} \mathrm{O}(4: 1)$ & 20 \\
\hline 6 & $\mathrm{MeOH}: \mathrm{H}_{2} \mathrm{O}(4: 1)$ & 37 \\
\hline 6 & $\mathrm{CH}_{3} \mathrm{CN}: \mathrm{H}_{2} \mathrm{O}(4: 1)$ & 2 \\
\hline 7 & THF: $\mathrm{H}_{2} \mathrm{O}(4: 1)$ & 39 \\
\hline 8 & 1,4-dioxane: $\mathrm{H}_{2} \mathrm{O}(4: 1)$ & 44 \\
\hline 9 & Acetone: $\mathrm{H}_{2} \mathrm{O}(4: 1)$ & 26 \\
\hline 10 & DMA & 35 \\
\hline 12 & DMA: $\mathrm{H}_{2} \mathrm{O}(9: 1)$ & 45 \\
\hline 13 & DMA: $\mathrm{H}_{2} \mathrm{O}(3: 2)$ & $47^{\mathrm{b}}$ \\
\hline 14 & DMA: $\mathrm{H}_{2} \mathrm{O}(7: 3)$ & $47^{\mathrm{b}}$ \\
\hline 15 & DMA: $\mathrm{H}_{2} \mathrm{O}(4: 1,0.1 \mathrm{M})$ & 51 \\
\hline 16 & DMA: $\mathrm{H}_{2} \mathrm{O}(4: 1,0.05 \mathrm{M})$ & 36 \\
\hline
\end{tabular}

${ }^{a}$ Yields of 5a determined by ${ }^{1} \mathrm{H}$ NMR analysis of the crude mixture using 1,2,3-trimethoxybenzene as the internal standard; ${ }^{b}$ the reaction mixture was found partially insoluble in the solvent system; yields of isolated $\mathbf{5 a}$ are reported in brackets 


\section{C.5 Investigation of other radical precursors}

During the optimization studies, other radical precursors were tested within our reaction system. In particular, radical precursors $\mathbf{2 b - e}$ demonstrated the formation of an EDA-complex with 1a but afforded poor yields or complex mixture of products (Figure S2a). Other commonly used radical precursors such as sulfones $\mathbf{2 f}$ and redox active esters $\mathbf{2 g}$ did not show the formation of EDAcomplex with 1a (Figure S2b).

(a) Radical precursors making EDA-complex with Boc-Trp-Ala-OMe (1a)<smiles>Cc1ccc(C(=O)CBr)cc1</smiles>

$4 \%$ yield

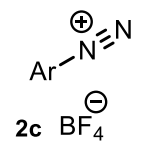

complex mixture

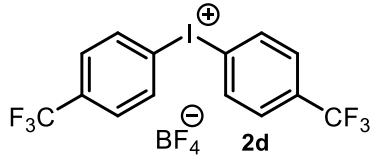

$20 \%$ yield

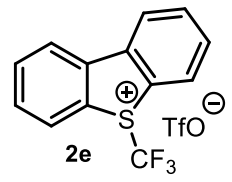

$40 \%$

(b) Common radical precursors making no EDA-complex with 1a<smiles>[R]S(=O)(=O)c1nnnn1-c1ccccc1</smiles>

$2 f$<smiles>Cc1ccc2c(c1)C(=O)N(OC(=O)Cc1ccc(C(F)(F)F)cc1)C2=O</smiles>

2g

Figure S2. Investigation of other radical precursors

\section{Syntheses of dipeptide, tripeptide and tetrapeptide starting materials $(1 \mathrm{a}-10)^{1}$}

Except otherwise stated, the dipeptide starting materials 1 were synthesized using the following procedure.

\section{D.1 General procedure for amide coupling}

1. Removal of $\mathbf{H C l}$ salt. The $\mathrm{HCl}$ salts of the amino acid methyl ester starting materials were removed by extractions using a saturated aqueous solution of $\mathrm{Na}_{2} \mathrm{CO}_{3}$. As a typical procedure, $1 \mathrm{~g}$ of $\mathrm{HCl}$-amino acid-OMe was dissolved in $\mathrm{CH}_{2} \mathrm{Cl}_{2}(30 \mathrm{~mL})$ and washed with a $\mathrm{Na}_{2} \mathrm{CO}_{3}$ aqueous solution $\left(3 \times 100 \mathrm{~mL}\right.$ ). The organic layer was dried over $\mathrm{MgSO}_{4}$, and concentrated under rotary evaporation. The resulting and usually volatile free amino acid methyl ester was then used without further purification into the following amide coupling reaction.

2. Amide coupling. The freshly prepared amino acid methyl ester (1 equiv.) was added into a round bottom flask and dissolved in DMF (0.5 M). Boc-Trp-OH (1 equiv.), HBTU (1.2 equiv.) and DIPEA (2 equiv.) were added sequentially and the reaction mixture was stirred at room temperature for 2 hours. The resulting mixture was diluted with EtOAc $(500 \mathrm{~mL})$ and washed with an aqueous solution of $\mathrm{HCl}(1 \mathrm{~N}, 400 \mathrm{~mL})$, a saturated aqueous solution of $\mathrm{NaHCO}_{3}(300$ $\mathrm{mL})$, and brine $(300 \mathrm{~mL})$. The organic layer was dried with $\mathrm{MgSO}_{4}$, concentrated by rotary evaporation on silica gel, and purified by silica gel column chromatography.

\section{D.2 Characterization data of compounds 1a-1o}<smiles>COC(=O)C([14CH3])NC(=O)[C@H](Cc1c[nH]c2ccccc12)NC(=O)c1ccccc1</smiles>

reports. ${ }^{1}$
Boc-L-Trp-L-Ala-OMe (1a) was synthesized following the amide coupling procedure at $19 \mathrm{mmol}$ scale and purified by silica gel column chromatography using hexane/EtOAc $(1: 1)$ as the eluting solvent. Dipeptide 1 was obtained as a white solid (5.7 g, 77\%). Characterization data were in accordance with previously published 


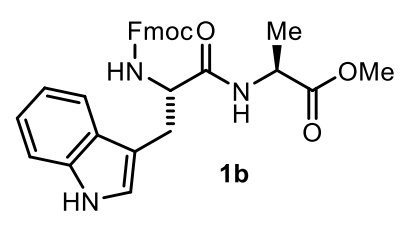

Fmoc-L-Trp-L-Ala-OMe (1b) was synthesized following the amide coupling procedure at $7.4 \mathrm{mmol}$ scale and purified by silica gel column chromatography using hexane/EtOAc (1:1) as eluting solvent. Dipeptide 2 was obtained as a white solid (630 mg, 17\%).

${ }^{1} \mathrm{H}$ NMR (400 MHz, $\left.\mathrm{C}_{3} \mathrm{D}_{7} \mathrm{NO}\right): \delta 10.9(\mathrm{~s}, 1 \mathrm{H}), 8.49(\mathrm{~d}, J=6.8 \mathrm{~Hz}$, $1 \mathrm{H}), 7.92(\mathrm{~d}, J=7.6 \mathrm{~Hz}, 2 \mathrm{H}), 7.71(\mathrm{dd}, J=11.6,7.8 \mathrm{~Hz}, 3 \mathrm{H}), 7.53-7.22(\mathrm{~m}, 7 \mathrm{H}), 7.11(\mathrm{t}, J=$ $7.5 \mathrm{~Hz}, 1 \mathrm{H}), 7.03(\mathrm{t}, J=7.5 \mathrm{~Hz}, 1 \mathrm{H}), 4.72-4.39(\mathrm{~m}, 2 \mathrm{H}), 4.28-4.07(\mathrm{~m}, 3 \mathrm{H}), 3.69(\mathrm{~s}, 3 \mathrm{H}), 3.35$ (dt, $J=14.6,4.0 \mathrm{~Hz}, 1 \mathrm{H}), 3.26-3.06(\mathrm{~m}, 1 \mathrm{H}), 1.37(\mathrm{~d}, J=7.3 \mathrm{~Hz}, 3 \mathrm{H})$.

${ }^{13} \mathrm{C} \mathrm{NMR}\left(101 \mathrm{MHz}, \mathrm{C}_{3} \mathrm{D}_{7} \mathrm{NO}\right): \delta 174.3,173.2,157.2,145.30,145.27,142.2,137.9,128.9,128.7$, 128.2, 126.6, 126.5, 125.2, 122.0, 121.1, 119.6, 119.5, 112.4, 111.6, 67.3, 56.8, 52.7, 49.1, 48.1, 29.3, 17.9.

HRMS (ESI) $m / z:[\mathrm{M}+\mathrm{Na}]^{+}$calcd for $\mathrm{C}_{30} \mathrm{H}_{29} \mathrm{~N}_{3} \mathrm{NaO}_{5}$ 534.1999; found 534.2005.<smiles>COC(=O)C(NC(=O)[C@H](Cc1c[nH]c2ccccc12)NC(=O)OCc1ccccc1)C(C)C</smiles>

previously published reports ${ }^{1}$

Boc-L-Trp-L-Val-OMe (1c) was synthesized following the amide coupling procedure at $1.6 \mathrm{mmol}$ scale and purified by silica gel column chromatography using hexane/EtOAc (gradient from 2:8 to 1:1) as eluting solvent. Dipeptide 5 was obtained as a pale yellow solid $(628 \mathrm{mg}, 91 \%)$. Characterization data were in accordance with<smiles>CCC(C)C(NC(=O)[C@H](Cc1c[nH]c2ccccc12)NC(=O)c1ccccc1)C(=O)OC</smiles><smiles>COC(=O)C(CC(C)C)NC(=O)[C@H](Cc1c[nH]c2ccccc12)NC(=O)c1ccccc1</smiles>

Boc-L-Trp-L-Ile-OMe (1d) was synthesized following the amide coupling procedure at $5 \mathrm{mmol}$ scale and purified by silica gel column chromatography using hexane/EtOAc $(1: 2)$ as eluting solvent. Dipeptide 5 was obtained as a pale yellow solid (1.9852 g, 92\%). Characterization data were in accordance with previously published reports. ${ }^{1}$

Boc-L-Trp-L-Leu-OMe (1e) was synthesized following the amide coupling procedure at $5 \mathrm{mmol}$ scale and purified by Silica gel column chromatography using hexane/EtOAc (1:2) as eluting solvent. Dipeptide 1e was obtained as a pale yellow solid (1.8356 g, $85 \%$ ). Characterization data were in accordance with previously published reports. ${ }^{1}$<smiles>COC(=O)C(Cc1ccccc1)NC(=O)[C@H](Cc1c[nH]c2ccccc12)NC(=O)c1ccccc1</smiles>

Boc-L-Trp-L-Phe-OMe (1f) was synthesized following the amide coupling procedure at a $1.6 \mathrm{mmol}$ scale and purified by silica gel column chromatography using hexane/EtOAc (7:3) as eluting solvent. Dipeptide 1f was obtained as a white solid (745 mg, 100\%). Characterization data were in accordance with previously published reports. ${ }^{1}$

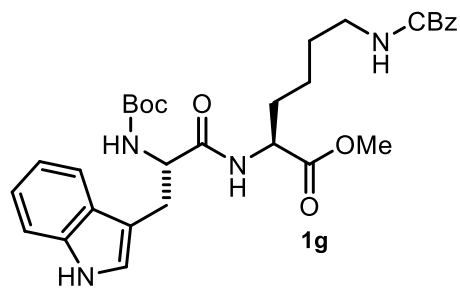

Boc-Trp-Lys(CBz)-OMe (1g) was synthesized following the amide coupling procedure at a $2.4 \mathrm{mmol}$ scale and purified by silica gel column chromatography using hexane/EtOAc (gradient from 1:1 to 8:2) as eluting solvent. Dipeptide 1g was obtained as a white solid (1.16 g, 83\%).

${ }^{1} \mathrm{H}$ NMR $\left(500 \mathrm{MHz}, \mathrm{CDCl}_{3}\right): \delta 9.16(\mathrm{~s}, 1 \mathrm{H}), 7.64(\mathrm{~d}, J=8.0 \mathrm{~Hz}$, 1H), $7.44-7.29(\mathrm{~m}, 6 \mathrm{H}), 7.18$ (ddd, $J=8.1,7.0,1.2 \mathrm{~Hz}, 1 \mathrm{H}), 7.15-7.09(\mathrm{~m}, 1 \mathrm{H}), 7.03(\mathrm{~s}, 1 \mathrm{H})$, 6.48 (br s, 1H), $5.23-5.05$ (m, 3H), 4.93 (br s, 1H), 4.51 (td, $J=6.9,4.4 \mathrm{~Hz}, 2 \mathrm{H}), 3.65$ (s, 3H), $3.46(\mathrm{~d}, J=13.1 \mathrm{~Hz}, 1 \mathrm{H}), 3.14(\mathrm{dd}, J=14.5,6.5 \mathrm{~Hz}, 2 \mathrm{H}), 3.07-2.95(\mathrm{~m}, 1 \mathrm{H}), 2.00-1.50(\mathrm{~m}$, $3 \mathrm{H}), 1.45(\mathrm{~s}, 9 \mathrm{H}), 1.41-1.30(\mathrm{~m}, 1 \mathrm{H}), 1.11-0.82(\mathrm{~m}, 2 \mathrm{H})$. 
${ }^{13} \mathrm{C}$ NMR $\left(101 \mathrm{MHz}, \mathrm{CDCl}_{3}\right): \delta 172.2,171.6,156.9,155.6,136.6,136.4,128.7,128.4,128.3$, $127.8,123.8,122.1,119.8,118.6,111.6,109.8,80.4,67.0,55.7,52.5,52.1,41.0,31.8,29.8,28.4$, 27.8, 21.9 .

HRMS (ESI) $m / z:[\mathrm{M}+\mathrm{Na}]^{+}$calcd for $\mathrm{C}_{31} \mathrm{H}_{41} \mathrm{~N}_{4} \mathrm{O}_{7} \mathrm{Na}$ 581.2970; found 581.2971.<smiles>COC(=O)C(CC(C)=O)NC(=O)C(Cc1c[nH]c2ccccc12)NC(=O)c1ccccc1</smiles>

published reports. ${ }^{1}$<smiles>CC(=O)C(Cc1c[nH]cn1)NC(=O)[C@H](Cc1c[nH]c2ccccc12)NC(=O)c1ccccc1</smiles>

Boc-L-Trp-L-His-OMe (1i) was obtained after four steps. First, His(Tr)-L-OMe was synthesized following a previously reported twostep procedure through ditrytilation and selective mono-deprotection sequence of methyl $D$-histidinate dihydrochloride. ${ }^{2}$ Then, His(Tr)-LOMe was coupled with Boc-L-Trp following the amide coupling procedure to obtain Boc-L-Trp-L-His(Tr)-OMe. The final step was the removal of trityl group under acidic conditions. ${ }^{3}$<smiles>CC(=O)OCc1c[nH]cn1</smiles>

$$
\begin{aligned}
& \underset{\mathrm{TrCl}(2.0 \text { equiv) }}{\mathrm{Et}_{3} \mathrm{~N}(3.0 \text { equiv })} \\
& \underset{0{ }^{\circ} \mathrm{C} \text { to } \mathrm{RT}, 16 \mathrm{~h}}{\mathrm{CH}_{2} \mathrm{Cl}_{2}}
\end{aligned}
$$<smiles>[3H]NC(Cc1cn([In])cn1)C(C)=O</smiles>

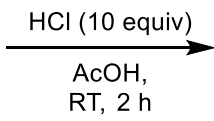<smiles>[M]OC(=O)[C@H](Cc1cn(I)cn1)NC(=O)[C@H](Cc1c[nH]c2ccccc12)NC(=O)OCc1ccccc1</smiles><smiles>COC(=O)[C@H](Cc1c[nH]cn1)NC(=O)[C@H](Cc1c[nH]c2ccccc12)NC(=O)O[Na]</smiles>

$55 \%$<smiles>COC(=O)C(N)Cc1cn([In])cn1</smiles>

His(Tr)-L-OMe (S2). Dichloromethane $(20 \mathrm{~mL})$ and methyl $D$-histidinate dihydrochloride ( $3.0 \mathrm{~g}, 12.4 \mathrm{mmol})$ were added into a round bottom flask. The stirred mixture was cooled in an ice bath. In a separated flask containing a solution of trityl chloride $(6.91 \mathrm{~g}, 24.8 \mathrm{mmol})$ in dichloromethane $(20 \mathrm{~mL})$ was slowly added $\mathrm{Et}_{3} \mathrm{~N}(5.2 \mathrm{~mL}, 37.2 \mathrm{mmol})$. The resulting solution was then added to the stirred methyl ester mixture and stir was continued overnight at ambient temperature. The reaction was then washed with water $(3 \times 50 \mathrm{~mL})$. The organic layer was dried $\left(\mathrm{Na}_{2} \mathrm{SO}_{4}\right)$, filtered, and then concentrated in vacuo. The resulting product $\mathbf{S 1}$ was used in the next step without further purification.

Acetic acid $(32 \mathrm{~mL})$ and conc. $\mathrm{HCl}(2.9 \mathrm{~mL})$ were added to a round bottom flask with ditritylHis-OMe S1 (8.0 g, $12.24 \mathrm{mmol})$. The reaction was allowed to stir for $2 \mathrm{~h}$ at ambient temperature. Reaction mixture was then concentrated in vacuo to obtain a white suspension, which was then suspended in water and diethyl ether. The phases were separated. The aqueous phase was then washed with diethyl ether $(2 \times 30 \mathrm{~mL})$. Sodium carbonate was carefully added to the aqueous phase to basify. The aqueous phase was extracted with dichloromethane $(2 \times 100 \mathrm{~mL})$. The organic layer was dried $\left(\mathrm{Na}_{2} \mathrm{SO}_{4}\right)$, filtered, and then concentrated in vacuo. Remaining acetic acid was removed by azeotrope evaporation with toluene $(3 \times 100 \mathrm{~mL})$, to afford the title compound S2 as a pale yellow oil $(2.7 \mathrm{~g}, 54 \%)$. 
Characterization data were in accordance with previously published report. ${ }^{\text {Error! Bookmark not defined. }}$<smiles>CCCn1cnc(C[C@H](NC(=O)[C@H](Cc2c[nH]c3ccccc23)NC(=O)[O-])C(C)=O)c1</smiles>

Boc-L-Trp-L-His(Tr)-OMe (S3) was synthesized following the amide coupling procedure using $\mathrm{His}(\mathrm{Tr})-\mathrm{L}-\mathrm{OMe}(\mathbf{S} 2)$ at $1.5 \mathrm{mmol}$ scale and purified by silica gel column chromatography using $\mathrm{CH}_{2} \mathrm{Cl}_{2} / \mathrm{MeOH}$ (98:2) as eluting solvent. The resulting product was further washed by extraction in $\mathrm{Et}_{2} \mathrm{O}(50 \mathrm{~mL})$ using a 1:1 mixture of $\mathrm{H}_{2} \mathrm{O} /$ brine $(3 \times 50 \mathrm{~mL}$ ) to remove the remaining DMF. After drying the organic phase with $\mathrm{MgSO}_{4}$ and solvent removal in vacuo, Boc-L-TrpL-His(Tr)-OMe (S3) dipeptide was obtained as a white solid $(0.59 \mathrm{~g}, 55 \%$ yield).

${ }^{1} \mathrm{H} \mathrm{NMR}\left(400 \mathrm{MHz}, \mathrm{CDCl}_{3}\right): \delta 8.06(\mathrm{~s}, 1 \mathrm{H}), 7.66(\mathrm{~d}, J=7.9 \mathrm{~Hz}, 1 \mathrm{H}), 7.46-77.22(\mathrm{~m}, 11 \mathrm{H}), 7.21$ $-7.15(\mathrm{~m}, 1 \mathrm{H}), 7.14-7.01(\mathrm{~m}, 9 \mathrm{H}), 6.39(\mathrm{~s}, 1 \mathrm{H}), 5.27(\mathrm{br} \mathrm{s}, 1 \mathrm{H}), 4.74(\mathrm{dt}, J=7.7,4.9 \mathrm{~Hz}, 1 \mathrm{H})$, $4.52(\mathrm{br} \mathrm{s}, 1 \mathrm{H}), 3.56(\mathrm{~s}, 3 \mathrm{H}), 3.36-3.12(\mathrm{~m}, 2 \mathrm{H}), 2.97(\mathrm{dd}, J=14.6,5.1 \mathrm{~Hz}, 1 \mathrm{H}), 2.90-2.73(\mathrm{~m}$, $1 \mathrm{H}), 1.37(\mathrm{~s}, 9 \mathrm{H})$.

${ }^{13} \mathrm{C} \mathrm{NMR}\left(101 \mathrm{MHz}, \mathrm{CDCl}_{3}\right): \delta 171.6,171.5,155.4,142.3,138.5,136.2,136.0,129.9,128.3$, 128.2, 127.9, 123.5, 122.0, 119.7, 119.2, 111.1, 110.7, 79.7, 75.5, 55.1, 55.6, 52.2, 29.9, 28.4 .

HRMS (ESI) $m / z:[\mathrm{M}+\mathrm{H}]^{+}$calcd for $\mathrm{C}_{42} \mathrm{H}_{44} \mathrm{~N}_{5} \mathrm{O}_{5} 698.3337$; found 698.3364 .<smiles>CC(=O)C(Cc1c[nH]cn1)NC(=O)[C@H](Cc1c[nH]c2ccccc12)NC(=O)O</smiles>

Boc-L-Trp-L-His-OMe (1i) was synthesized by a trityl deprotection sequence following a previous report. ${ }^{3}$

Boc-Trp-His(Tr)-OMe $\mathbf{S 3}$ (136 mg, $0.300 \mathrm{mmol}$ ) and 90\% acetic acid in water $(1 \mathrm{~mL})$ were added to a $50 \mathrm{~mL}$ round-bottom flask. The reaction mixture was heated at $60^{\circ} \mathrm{C}$ for 90 minutes. Toluene was added to the reaction mixture and $\mathrm{AcOH}$ was removed in vacuo $(3 \mathrm{x}$ $50 \mathrm{~mL}$ ). The reaction mixture was concentrated and purified by silica gel column chromatography using $\mathrm{CH}_{2} \mathrm{Cl}_{2} / \mathrm{MeOH}$ (gradient from 95:5 to 9:1) as eluting solvent, to afford the desired product $\mathbf{1 i}$ as a white solid (125 $\mathrm{mg}, 92 \%)$.

Characterization data were in accordance with previously published report. ${ }^{4}$<smiles>COC(=O)C(CO)NC(=O)C(Cc1c[nH]c2ccccc12)NC(=O)c1ccccc1</smiles>

Boc-L-Trp-L-Ser-OMe (1j) was synthesized following a previously reported procedure. ${ }^{7}$

Boc-Trp-OH (609 mg, $2 \mathrm{mmol})$ was dissolved in acetonitrile $(20 \mathrm{~mL})$ and put in an ice bath. HOBt (270 mg, $2 \mathrm{mmol})$, DCC (413 mg, 2 mmol), L-Serine methyl ester (311 mg, $2 \mathrm{mmol}$ ), and triethylamine $(0.56 \mathrm{~mL}, 4 \mathrm{mmol})$ were added waiting for about $2 \mathrm{~min}$ between each addition. The mixture was left stirring at ambient temperature overnight. The resulting solid was filtered and the solvent removed under reduced pressure. Acetone was added, and the mixture was stored in the freezer for 2 hours. The urea was filtered again. Evaporation at reduced pressure gave a residue that was partitioned between ethyl acetate $(50 \mathrm{~mL})$ and an aqueous solution of $\mathrm{KHSO}_{4}(1 \mathrm{M}, 30 \mathrm{~mL})$. The organic phase was thoroughly washed with $\mathrm{KHSO}_{4}(3 \times 30 \mathrm{~mL})$, an aqueous solution of $\mathrm{NaHCO}_{3}$ $(1 \mathrm{M}, 3 \times 30 \mathrm{~mL})$, and brine $(3 \times 30 \mathrm{~mL})$ and dried with $\mathrm{MgSO}_{4}$. Removal of the solvent afforded compounds $\mathbf{1} \mathbf{j}$ as a white solid without further purification $(784 \mathrm{mg}, 97 \%)$.

${ }^{1} \mathrm{H} \mathrm{NMR}\left(400 \mathrm{MHz}, \mathrm{CDCl}_{3}\right): \delta 8.20(\mathrm{~s}, 1 \mathrm{H}), 7.64(\mathrm{~d}, J=8.0 \mathrm{~Hz}, 1 \mathrm{H}), 7.36(\mathrm{~d}, J=8.1 \mathrm{~Hz}, 1 \mathrm{H})$, 7.20 (ddd, $J=8.1,7.0,1.2 \mathrm{~Hz}, 1 \mathrm{H}), 7.17-6.97(\mathrm{~m}, 2 \mathrm{H}), 6.69$ (d, $J=7.0 \mathrm{~Hz}, 1 \mathrm{H}), 5.14$ (brs, $1 \mathrm{H})$, 
$4.49(\mathrm{dt}, J=7.0,3.5 \mathrm{~Hz}, 1 \mathrm{H}), 4.41(\mathrm{q}, J=6.6 \mathrm{~Hz}, 1 \mathrm{H}), 3.78(\mathrm{ddd}, J=11.2,7.0,3.7 \mathrm{~Hz}, 2 \mathrm{H}), 3.69$ (s, 3H), 3.37 (dd, $J=14.6,5.9 \mathrm{~Hz}, 1 \mathrm{H}), 3.19$ (dd, $J=14.6,6.7 \mathrm{~Hz}, 1 \mathrm{H}), 2.47$ (brs, 1H), 1.42 (s, 9H).

${ }^{13} \mathrm{C} \mathrm{NMR}\left(101 \mathrm{MHz}, \mathrm{CDCl}_{3}\right): \delta 172.4,170.6,155.9,136.4,127.6,123.6,122.2,119.6,118.7$, $111.5,110.1,80.6,62.7,60.6,55.5,55.0,52.7,49.5,33.8,28.4,28.1,25.6,25.0$.

HRMS (ESI) $m / z:[\mathrm{M}+\mathrm{Na}]^{+}$calcd for $\mathrm{C}_{20} \mathrm{H}_{27} \mathrm{~N}_{3} \mathrm{NaO}_{6} 428.1792$; found 428.1780 .<smiles>COC(=O)C(Cc1ccc(O)cc1)NC(=O)[C@H](Cc1c[nH]c2ccccc12)NC(=O)c1ccccc1</smiles>

Boc-L-Trp-L-Tyr-OMe (1k) were prepared according to previously published procedures. ${ }^{6}$

(Tert-butoxycarbonyl)-L-tryptophan (1,52 g, $5 \mathrm{mmol})$ and methyl L-tyrosinate $(0,98 \mathrm{~g}, 5 \mathrm{mmol})$ were combined in DCM $(15 \mathrm{~mL})$ and the reaction mixture was cooled to $0{ }^{\circ} \mathrm{C}$. Following sequential addition of dicyclohexylmethanediimine $(0,94 \mathrm{~mL}, 6,00 \mathrm{mmol})$, $1 \mathrm{H}-$ benzo[d][1,2,3]triazol-1-ol $(0,81 \mathrm{~g}, \quad 6 \mathrm{mmol})$ and 4 methylmorpholine $(1,653 \mathrm{ml}, 15 \mathrm{mmol})$ at this temperature, the reaction mixture was allowed to warm up to ambient temperature and stirring continued overnight. The suspension was then filtered over a short pad of celite and washed with $30 \mathrm{~mL}$ DCM. The filtrate was washed with $1 \mathrm{~N}$ $\mathrm{HCl}$, sat. sodium bicarbonate, and brine. The organic layer was dried over magnesium sulfate and concentrated. The residue was purified by silica gel column chromatography (hexanes:EtOAc $1: 1)$ to afford the title compound $1 \mathbf{k}(1.85 \mathrm{~g}, 77 \%)$. Characterization data were in accordance with previously published report. ${ }^{6}$<smiles>COC(=O)[C@H](CCSC)NC(=O)[C@H](Cc1c[nH]c2ccccc12)NC(=O)OCc1ccccc1</smiles><smiles>COC(=O)[C@H](Cc1c[nH]c2ccccc12)NC(=O)[C@H](Cc1c[nH]c2ccccc12)NC(=O)OCc1ccccc1</smiles>

Boc-L-Trp-L-Met-OMe (11) was synthesized following the amide coupling procedure at a $5.1 \mathrm{mmol}$ scale and purified by silica gel column chromatography using hexane/EtOAc (3:2) as the eluting solvent. Dipeptide $1 \mathrm{l}$ was obtained as a white solid (1.49 g, 65\%). Characterization data were in accordance with previously published report. $^{5}$

Boc-L-Trp-L-Trp-OMe (1m) was synthesized following the amide coupling procedure at a $1.6 \mathrm{mmol}$ scale and purified by silica gel column chromatography using hexane/EtOAc (1:1) as eluting solvent. Dipeptide 1m was obtained as a white solid (734 mg, 91\%). Characterization data were in accordance with previously published report. ${ }^{1}$<smiles>CC(C)(C)OC(=O)CNC(=O)C[C@H](Cc1c[nH]c2ccccc12)C(=O)O</smiles>

Boc-L-Gly-L-Trp-OMe (1n) was synthesized following the amide coupling procedure at a $10 \mathrm{mmol}$ scale and purified by silica gel column chromatography using hexane/EtOAc (1:1) as eluting solvent. Dipeptide 1n was obtained as a white solid (3.0765 g, 82\%). Characterization data were in accordance with previously published report. ${ }^{1}$<smiles>COC(=O)[C@H](Cc1c[nH]c2ccccc12)NC(=O)CNC(=O)CNC(=O)OC(C)(C)C</smiles>

Boc-Gly-Gly-Trp-OMe (10) was synthesized following the amide coupling procedure at $1 \mathrm{mmol}$ scale and purified by silica gel column chromatography using hexane/EtOAc (gradient from 1:2 to pure EA) as eluting solvent. Tripeptide 10 was obtained as a yellow solid $(236 \mathrm{mg}, 55 \%){ }^{8}$ 
${ }^{1} \mathrm{H} \mathrm{NMR}(300 \mathrm{MHz}, \mathrm{CDCl} 3) \delta 8.80(\mathrm{~s}, 1 \mathrm{H}), 7.46(\mathrm{~d}, J=7.8 \mathrm{~Hz}, 1 \mathrm{H}), 7.32(\mathrm{~d}, J=8.1 \mathrm{~Hz}, 1 \mathrm{H})$, 7.11 (dddd, $J=24.0,8.0,7.0,1.2 \mathrm{~Hz}, 2 \mathrm{H}), 7.00-6.83(\mathrm{~m}, 3 \mathrm{H}), 5.24(\mathrm{~s}, 1 \mathrm{H}), 4.85(\mathrm{~d}, J=6.9 \mathrm{~Hz}$, $1 \mathrm{H}), 3.67(\mathrm{~d}, J=5.8 \mathrm{~Hz}, 7 \mathrm{H}), 3.28(\mathrm{~d}, J=5.7 \mathrm{~Hz}, 2 \mathrm{H}), 1.45(\mathrm{~s}, 9 \mathrm{H})$.

${ }_{13}^{13} \mathrm{C}$ NMR $(126 \mathrm{MHz}, \mathrm{MeOD}) \delta 173.7,173.0,171.3,158.5,138.0,128.7,124.7,122.4,119.9$, 119.1, 112.3, 110.4, 80.9, 61.5, 54.9, 52.7, 44.8, 43.2, 28.7, 28.4, 20.8, 14.4.

HRMS (ESI) $m / z:$ : $\mathrm{M}-\mathrm{H}]^{-}$calcd for $\mathrm{C}_{21} \mathrm{H}_{27} \mathrm{~N}_{4} \mathrm{O}_{6}$ 431.1936; found 431.1936 .<smiles>COC(=O)C(CC(C)C)NC(=O)[C@H](Cc1c[nH]c2ccccc12)NC(=O)CNC(=O)CNC(=O)c1ccccc1</smiles>

${ }^{1} \mathrm{H} \mathrm{NMR}\left(300 \mathrm{MHz}, \mathrm{CDCl}_{3}\right) \delta 8.64(\mathrm{~s}, 1 \mathrm{H}), 7.56(\mathrm{~d}, J=7.8 \mathrm{~Hz}, 1 \mathrm{H}), 7.30(\mathrm{~d}, J=8.0 \mathrm{~Hz}, 1 \mathrm{H})$, $7.17-6.96(\mathrm{~m}, 5 \mathrm{H}), 5.38(\mathrm{~s}, 1 \mathrm{H}), 4.85(\mathrm{q}, J=6.9 \mathrm{~Hz}, 1 \mathrm{H}), 4.53(\mathrm{~d}, J=6.9 \mathrm{~Hz}, 1 \mathrm{H}), 3.68(\mathrm{~s}, 7 \mathrm{H})$, $3.32-3.12(\mathrm{~m}, 2 \mathrm{H}), 1.46(\mathrm{~s}, 12 \mathrm{H}), 0.86(\mathrm{~d}, J=5.6 \mathrm{~Hz}, 6 \mathrm{H})$.

${ }^{13} \mathrm{C}$ NMR $(126 \mathrm{MHz}, \mathrm{MeOD}) \delta 174.3,173.9,173.1,171.3,158.5,138.0,128.9,124.7,122.4$, $119.8,119.3,112.3,110.7,80.9,61.5,55.4,52.6,52.3,44.8,43.5,41.5,28.7,25.8,23.3,21.9$, 14.5.

HRMS (ESI) m/z: [M-H] $]^{-}$calcd for $\mathrm{C}_{27} \mathrm{H}_{38} \mathrm{~N}_{5} \mathrm{O}_{7}$ 544.2777; found 544.2765.

\section{E. Syntheses of pyridinium salts 4}

Except otherwise stated, the pyridinium salts 4 were synthesized using the following general procedure. Compounds 4a, KS-Leu-OMe, KS-Ala-OMe, KS-Ala-OBn, ,KS-Leu-KS, KS-PheOMe, Met-OMe, KS-Phe-OMe (KS: Katritzky salts) were prepared according to previously published procedures. ${ }^{9}$

\section{E.1 General procedure}

The amino ester hydrochloride (1.2 equiv.) was dissolved in ethanol $(1 \mathrm{M})$ and triethylamine (1.2 equiv.) was added. The resulting suspension was stirred at ambient temperature for $30 \mathrm{~min}$, followed by addition of triphenylpyrylium tetrafluoroborate (1 equiv.). When free amino esters were used, the reactants were directly combined in ethanol without using triethylamine. The red reaction mixture was then refluxed for 4 hours and subsequently allowed to cool down to ambient temperature. $\mathrm{Et}_{2} \mathrm{O}$ was added and the mixture was stirred for $1 \mathrm{~h}$. The resulting solid was collected by filtration and thoroughly washed with $\mathrm{H}_{2} \mathrm{O}$ and $\mathrm{Et}_{2} \mathrm{O}$. The remaining solid was then dried under reduced pressure to afford the pyridinium salt $\mathbf{4}$ without further purification. If the precipitation did not take place, the solvent was remove under reduced pressure. The crude product was then dissolved in DCM and the organic layer was extracted with water $(3 \mathrm{x})$, dried over magnesium sulfate and concentrated. The crude product was purified by silica gel column chromatography using DCM/Acetone as eluent system.

\section{E.2 Characterization data of compounds $4 b-4 g$}


<smiles></smiles>
tetrafluoroborate (KS-Gly-OBn, $4 \mathrm{~b})$. Prepared according to the general procedure using benzyl glycinate hydrochloride salt $(968 \mathrm{mg}, 4.80 \mathrm{mmol})$, triphenylpyrylium tetrafluoroborate $(1.58 \mathrm{~g}, 4.00 \mathrm{mmol})$ and triethylamine $(669 \mu \mathrm{L}, 4.80 \mathrm{mmol})$.

The product was obtained after crystallization in $\mathrm{Et}_{2} \mathrm{O}$ as a pink powder (1.46 g, 67\% yield).

$\underline{{ }^{1} \mathrm{H} \mathrm{NMR}}\left(400 \mathrm{MHz}, \mathrm{CDCl}_{3}\right): \delta 7.94(\mathrm{~s}, 2 \mathrm{H}),, 7.79-7.29(\mathrm{~m}, 18 \mathrm{H}), 7.14-7.07(\mathrm{~m}, 2 \mathrm{H}), 5.12(\mathrm{~s}$, 2H), $4.98(\mathrm{~s}, 2 \mathrm{H})$.

${ }^{13} \mathrm{C}$ NMR $\left(101 \mathrm{MHz}, \mathrm{CDCl}_{3}\right): \delta 167.0,157.5,157.1,134.1,134.0,132.6,132.1,131.4,129.9$, 129.0, 128.8, 128.4, 126.3.68.4, 56.6.

${ }^{19} \mathrm{~F}$ NMR $\left(376 \mathrm{MHz}, \mathrm{CDCl}_{3}\right): \delta-153.44$.

HRMS (ESI) $m / z:\left[\mathrm{M}-\mathrm{BF}_{4}\right]^{+}$calcd for $\mathrm{C}_{32} \mathrm{H}_{26} \mathrm{NO}_{2}$ 456.1958; found 456.1944 .<smiles></smiles>

\section{1-(2-oxo-2-(2,2,2-trifluoroethoxy)ethyl)-2,4,6-triphenylpyridin-1-ium tetrafluoroborate (KS-Gly-OCH $\mathrm{CF}_{3}, 4 \mathrm{c}$ )}

Synthesized in two steps. Firstly, 2,2,2-trifluoroethyl glycinate hydrochloride was obtained following a reported procedure. ${ }^{7}$ Secondly, the title compound $\mathbf{4} \mathbf{c}$ was obtained following the general procedure using 2,2,2trifluoroethyl glycinate hydrochloride salt $(602.5 \mathrm{mg}, 3.11 \mathrm{mmol})$, triphenylpyrylium tetrafluoroborate $(1.03 \mathrm{~g}, 2.59 \mathrm{mmol})$ and triethylamine $(434 \mu \mathrm{L}, 3.11 \mathrm{mmol})$.

The product was purified by column chromatography (gradient DCM/Acetone 100:0 to 80:20) to obtain a yellow powder (799 mg, 58\%, mixture of rotamers).

${ }^{1} \mathrm{H}$ NMR (400 MHz, $\mathrm{CDCl}_{3}$, rotamers): $\delta 8.01-7.92(\mathrm{~m}, 2 \mathrm{H}), 7.88-7.66(\mathrm{~m}, 3 \mathrm{H}), 7.66-7.46(\mathrm{~m}$, $10 \mathrm{H}), 5.30$ (s, $1.56 \mathrm{H}$, rotamer), $5.15(\mathrm{~s}, 0.44 \mathrm{H}$, rotamer), 4.34 (q, $J=8.1 \mathrm{~Hz}, 1.60 \mathrm{H}$, rotamer), $4.00(\mathrm{q}, J=7.1 \mathrm{~Hz}, 0.40 \mathrm{H})$.

${ }^{13} \mathrm{C} \mathrm{NMR}\left(101 \mathrm{MHz}, \mathrm{CDCl}_{3}\right.$, rotamers): $\delta 167.1 \& 166.1,157.6 \& 157.5,157.4 \& 157.0,134.0$ $\& 133.8,132.8 \& 132.6,132.3 \& 132.0,131.7 \& 131.4,130.0 \& 129.9,128.4 \& 128.3,126.29 \&$ $126.25,62.9,61.3(\mathrm{q}, J=37.7 \mathrm{~Hz}), 56.7 \& 56.0$.

${ }^{19} \mathrm{~F} \mathrm{NMR}\left(376 \mathrm{MHz}, \mathrm{CDCl}_{3}\right): \delta-73.68,-153.29$.

HRMS (ESI) $m / z:$ : $\left[\mathrm{M}-\mathrm{BF}_{4}\right]^{+}$calcd for $\mathrm{C}_{27} \mathrm{H}_{21} \mathrm{~F}_{3} \mathrm{NO}_{2} 448.1519$; found 448.1530 .<smiles></smiles>

powder (350 mg, 14\%). 1-(1-(benzyloxy)-1-oxo-3-phenylpropan-2-yl)-2,4,6-triphenylpyridin1-ium tetrafluoroborate (KS-Phe-OBn, 4d)

Prepared according to the general procedure using benzyl phenylalaninate hydrochloride salt $(1.40 \mathrm{~g}, 4.80 \mathrm{mmol})$, triphenylpyrylium tetrafluoroborate $(1.58 \mathrm{~g}, 4,00 \mathrm{mmol})$ and triethylamine $(669 \mu \mathrm{L}, 4.80$ mmol). The product was obtained after purification by column chromatography (gradient DCM/Acetone 100:0 to 40:60) as a pale yellow 
$\underline{{ }^{1} \mathrm{H} \mathrm{NMR}}\left(300 \mathrm{MHz}, \mathrm{CDCl}_{3}\right): \delta 7.96(\mathrm{~s}, 2 \mathrm{H}), 7.90-7.63(\mathrm{~m}, 4 \mathrm{H}), 7.62-7.28(\mathrm{~m}, 14 \mathrm{H}), 7.23-$ $6.96(\mathrm{~m}, 5 \mathrm{H}), 6.82-6.65(\mathrm{~m}, 2 \mathrm{H}), 5.68(\mathrm{dd}, J=8.5,3.3 \mathrm{~Hz}, 1 \mathrm{H}), 5.11(\mathrm{~d}, J=11.8 \mathrm{~Hz}, 1 \mathrm{H}), 5.01$ (d, $J=11.8 \mathrm{~Hz}, 1 \mathrm{H}), 3.53$ (dd, $J=14.3,3.3 \mathrm{~Hz}, 1 \mathrm{H}), 2.73$ (dd, $J=14.3,8.6 \mathrm{~Hz}, 1 \mathrm{H}$ ).

${ }^{13} \mathrm{C}$ NMR $\left(126 \mathrm{MHz}, \mathrm{CDCl}_{3:}\right) \delta 168.1,157.3,136.8,134.1,133.7,132.7,132.5,131.5,129.8$, 129.3, 129.3, 129.2, 128.9, 128.7, 128.6, 127.2, 77.4, 77.2, 76.9, 70.7, 69.2, 37.8.

${ }^{19} \mathrm{~F} \mathrm{NMR}\left(376 \mathrm{MHz}, \mathrm{CDCl}_{3}\right) \delta-152.78,-152.84(\mathrm{~d}, J=1.9 \mathrm{~Hz})$.

HRMS (ESI) $m / z:\left[\mathrm{M}-\mathrm{BF}_{4}\right]^{+}$calcd for $\mathrm{C}_{39} \mathrm{H}_{32} \mathrm{NO}_{2}$ 546.2428; found 546.2413.

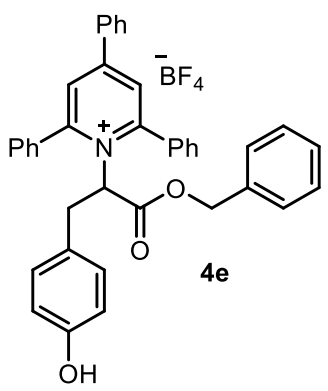

1-(1-(benzyloxy)-3-(4-hydroxyphenyl)-1-oxopropan-2-yl)-2,4,6triphenylpyridin-1-ium tetrafluoroborate (KS-Tyr-OBn, 4e). Prepared according to the general procedure using benzyl tyrosinate (1.30 $\mathrm{g}, 4.80 \mathrm{mmol})$ and triphenylpyrylium tetrafluoroborate $(1.58 \mathrm{~g}, 4,00$ mmol) without base.

The product was purified by column chromatography (gradient DCM/Acetone 100:0 to 35:65). As product was not separated from starting amino acid, the resulting product was taken in DCM, washed with

aqueous $\mathrm{HCl}(0.5 \mathrm{M})$, water then brine, dried over magnesium sulfate and concentrated to afford the pure product as a yellow powder $(600 \mathrm{mg}, 23 \%)$.

$\underline{{ }^{1} \mathrm{H} \mathrm{NMR}}\left(300 \mathrm{MHz}, \mathrm{CDCl}_{3}\right) \delta 8.63(\mathrm{~s}, 1 \mathrm{H}), 7.90(\mathrm{~s}, 2 \mathrm{H}), 7.87-7.79(\mathrm{~m}, 2 \mathrm{H}), 7.60-7.33(\mathrm{~m}$, $13 \mathrm{H}), 7.25-7.19(\mathrm{~m}, 2 \mathrm{H}), 6.76(\mathrm{~d}, J=8.5 \mathrm{~Hz}, 2 \mathrm{H}), 6.35(\mathrm{~d}, J=8.5 \mathrm{~Hz}, 2 \mathrm{H}), 5.59$ (dd, $J=7.6$, $6.1 \mathrm{~Hz}, 1 \mathrm{H}), 5.14(\mathrm{~d}, J=11.8 \mathrm{~Hz}, 1 \mathrm{H}), 5.05(\mathrm{~d}, J=11.9 \mathrm{~Hz}, 1 \mathrm{H}), 3.14(\mathrm{dd}, J=14.8,7.6 \mathrm{~Hz}, 1 \mathrm{H})$, $2.82(\mathrm{dd}, J=14.9,6.1 \mathrm{~Hz}, 1 \mathrm{H})$.

${ }^{13} \mathrm{C}$ NMR $\left(101 \mathrm{MHz}, \mathrm{CDCl}_{3}\right) \delta 167.7,157.4,156.8,133.7,133.2,133.0,132.2,131.9,130.0$, $129.5,129.4,129.2,129.0,128.7,124.9,116.7,77.5,77.2,76.8,71.3,69.2,36.3$.

${ }^{19} \mathrm{~F}$ NMR $\left(376 \mathrm{MHz}, \mathrm{CDCl}_{3}\right) \delta-152.78,-152.84(\mathrm{~d}, J=1.9 \mathrm{~Hz})$.

HRMS (ESI) $m / z:\left[\mathrm{M}-\mathrm{BF}_{4}\right]^{+}$calcd for $\mathrm{C}_{39} \mathrm{H}_{32} \mathrm{NO}_{3}$ 562.2377; found 562.2356.<smiles>CC(=O)c1ccccc1</smiles>

1-(1-(benzyloxy)- 5-methoxy-1,5-dioxopentan-2-yl)-2,4,6triphenylpyridin-1-ium tetrafluoroborate (KS-GluOMe-OBn, 4f)

The amino acid hydrochloride salt was prepared according to the following a 3-steps procedure:

To the corresponding benzyl $N$-Boc glutamate $(2.00 \mathrm{~g}, 5.93 \mathrm{mmol})$ in dichloromethane $(50 \mathrm{~mL})$ was added tetrafluoroborate benzotriazoletetramethyluronium $(2.09 \mathrm{~g}$, $6.52 \mathrm{mmol})$. The mixture was stirred at room temperature for $30 \mathrm{~min}$. Then methanol $(1.34 \mathrm{~mL}$, $32.2 \mathrm{mmol})$ and triethylamine $(3.47 \mathrm{~mL}, 24.9 \mathrm{mmol})$ were added. The mixture was stirred at room temperature for another 2 hours. Solvent was removed under reduced pressure, residue was taken in ethyl acetate and washed with water and brine. The organic layer was dried over $\mathrm{MgSO}_{4}$ and concentrated under reduced pressure to give the methyl ester of Boc-Glu-OBn as a colorless oil. This oil was taken in DCM $(10 \mathrm{~mL})$ and trifluoroacetic acid $(10 \mathrm{~mL})$ was added. The mixture was stirred at room temperature for 1 hour. After concentration under reduced pressure, the residue was dissolved in dichloromethane and washed twice with a saturated $\mathrm{Na}_{2} \mathrm{CO}_{3}$ solution. The 
organic layer was collected and treated with a $2 \mathrm{M} \mathrm{HCl}$ solution in diethyl ether. The precipitate of methylated benzyl glutamate hydrochloride salt $\mathrm{HCl} \cdot \mathrm{Glu}-\mathrm{OBn}$ was then dried and used without further purification $(1.04 \mathrm{~g}, 61 \%)$.

Having $\mathrm{HCl} \cdot \mathrm{Glu}-\mathrm{OBn}$ in hands, compound $\mathbf{4 f}$ was prepared according to the general procedure using benzyl methyl glutamate hydrochloride salt $(1.04 \mathrm{~g}, 3.61 \mathrm{mmol})$, triphenylpyrylium tetrafluoroborate $(1.19 \mathrm{~g}, 3.01 \mathrm{mmol})$ and triethylamine $(504 \mu \mathrm{L}, 3.61 \mathrm{mmol})$. $4 \mathbf{f}$ was obtained without further purification as a beige powder $(240 \mathrm{mg}, 13 \%)$.

${ }^{1} \mathrm{H}$ NMR $\left(300 \mathrm{MHz}, \mathrm{CDCl}_{3}\right) \delta 7.94(\mathrm{~s}, 2 \mathrm{H}), 7.85(\mathrm{dd}, J=8.2,7.6 \mathrm{~Hz}, 2 \mathrm{H}), 7.62-7.43(\mathrm{~m}, 12 \mathrm{H})$, $7.43-7.31(\mathrm{~m}, 4 \mathrm{H}), 7.31-7.18(\mathrm{~m}, 2 \mathrm{H}), 5.69(\mathrm{t}, J=5.75 \mathrm{~Hz} 1 \mathrm{H}), 5.15(\mathrm{dd}, J=29.8,11.9 \mathrm{~Hz}$, $2 \mathrm{H}), 3.44(\mathrm{~s}, 3 \mathrm{H}), 2.29-2.10(\mathrm{~m}, 3 \mathrm{H}), 2.10-1.92(\mathrm{~m}, 1 \mathrm{H}), 1.89(\mathrm{~s}, 1 \mathrm{H})$.

${ }^{13} \mathrm{C} \mathrm{NMR}\left(126 \mathrm{MHz}, \mathrm{CDCl}_{3}\right) \delta 172.2,168.2,157.3,134.0,133.9,132.7,132.5,131.6,129.8$, 129.3, 129.3, 129.04, 128.98, 128.7, 69.2, 68.1, 51.8, 30.8, 26.8 .

${ }^{19} \mathrm{~F} \mathrm{NMR}\left(471 \mathrm{MHz}, \mathrm{CDCl}_{3}\right) \delta-153.04,-153.09$.

$\underline{\text { HRMS (ESI) }} \mathrm{m} / z:\left[\mathrm{M}-\mathrm{BF}_{4}\right]^{+}$calcd for $\mathrm{C}_{36} \mathrm{H}_{32} \mathrm{NO}_{4}$ 542.2326; found 542.2307.<smiles></smiles>

1-(1-(benzyloxy)-6-(((benzyloxy)carbonyl)amino)-1-oxohexan-2yl)-2,4,6-triphenylpyridin-1-ium tetrafluoroborate (KS-Lys-CbzOBn, 4g)

Prepared according to the general procedure using benzyl $\mathrm{N}$-Cbzlysinate hydrochloride salt $(1.18 \mathrm{~g}, 2.90 \mathrm{mmol})$, triphenylpyrylium tetrafluoroborate $(957 \mathrm{mg}, 2.42 \mathrm{mmol})$ and triethylamine $(404 \mu \mathrm{L}, 2.90$ mmol).

The product was obtained after purification by column chromatography (gradient DCM/Acetone 100:0 to 65:35) as a lemon-yellow powder (750 mg, 42\%).

${ }^{1} \mathrm{H}$ NMR $\left(300 \mathrm{MHz}, \mathrm{CDCl}_{3}\right) \delta 7.91(\mathrm{~s}, 2 \mathrm{H}), 7.87-7.76(\mathrm{~m}, 2 \mathrm{H}), 7.76-6.96(\mathrm{~m}, 23 \mathrm{H}), 5.55(\mathrm{t}, J$ $=6.0 \mathrm{~Hz}, 1 \mathrm{H}), 5.38(\mathrm{t}, J=6.8 \mathrm{~Hz}, 1 \mathrm{H}), 5.21(\mathrm{~d}, J=11.9 \mathrm{~Hz}, 1 \mathrm{H}), 5.11(\mathrm{~d}, J=11.9 \mathrm{~Hz}, 1 \mathrm{H}), 5.05$ (s, 2H), $3.03(\mathrm{q}, J=6.3 \mathrm{~Hz}, 2 \mathrm{H}), 1.64(\mathrm{dt}, J=20.2,8.0 \mathrm{~Hz}, 2 \mathrm{H}), 1.30(\mathrm{~h}, J=6.4 \mathrm{~Hz}, 1 \mathrm{H}), 1.12$ (dq, $J=10.3,6.9,5.9 \mathrm{~Hz}, 3 \mathrm{H})$.

${ }^{13} \mathrm{C} \mathrm{NMR}\left(101 \mathrm{MHz}, \mathrm{CDCl}_{3}\right) \delta 168.4,157.1,156.8,137.1,133.9,133.8,132.6,132.5,131.5$, $129.8,129.3,129.04,128.95,128.6,128.5,128.4,128.0,127.9,77.5,77.2,76.8,69.5,69.0,66.2$, 40.0, 30.6, 28.6, 24.0.

${ }^{19} \mathrm{~F}$ NMR $\left(376 \mathrm{MHz}, \mathrm{CDCl}_{3}\right) \delta-152.78,-152.84(\mathrm{~d}, J=1.9 \mathrm{~Hz})$.

HRMS (ESI) $m / z:\left[\mathrm{M}-\mathrm{BF}_{4}\right]^{+}$calcd for $\mathrm{C}_{44} \mathrm{H}_{41} \mathrm{~N}_{2} \mathrm{O}_{4}$ 561.3061; found 561.3084 . 


\section{F. Photochemical alkylation of Trp-containing peptides}

\section{F1. General procedure}
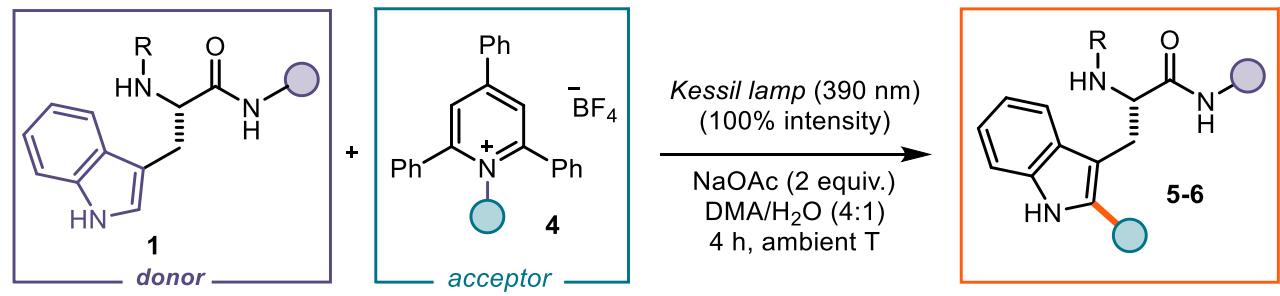

To a $5 \mathrm{~mL}$ argon purged glass vial, containing the dipeptide $\mathbf{1}(0.1 \mathrm{mmol})$, the pyridinium salt 4 $(0.3 \mathrm{mmol})$, and NaOAc $(0.2 \mathrm{mmol})$ was added $0.4 \mathrm{~mL}$ of argon-sparged DMA and $0.1 \mathrm{~mL}$ of argon-sparged distilled $\mathrm{H}_{2} \mathrm{O}$. The vial was sealed with parafilm and irradiated for $4 \mathrm{~h}$ with Kessil lamp PR160L (390 nm, 100\% intensity, $4 \mathrm{~cm}$ away from the vial, see the picture in the following section) at ambient temperature. The resulting crude mixture was diluted with EtOAc $(10 \mathrm{~mL})$, washed with brine $(2 \times 70 \mathrm{~mL})$, dried over $\mathrm{MgSO}_{4}$ and concentrated under rotary evaporation. The pure products were obtained after purification by silica gel column chromatography. Note that the freeze-pump-thaw cycle provided similar results and was therefore not needed.

\section{F2. Picture of the reaction setup}

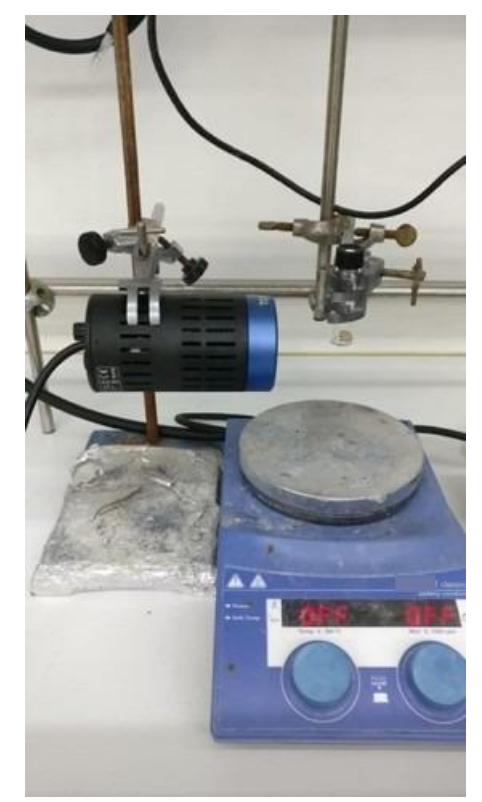

All photochemical reactions were carried out using a Kessil lamp PR160L (https://www.kessil.com/science/PR160L.php) with wavelength of peak intensity of $390 \mathrm{~nm}$, lamp intensity set to $100 \%$ and positioned at a distance of $4 \mathrm{~cm}$ from the reaction vessel, unless otherwise stated.

\section{F.3 Characterization data for compounds $5 a-5 p$ and $6 a-6 j$}<smiles>[Y6]C(NC(=O)C(Cc1sc2ccccc2c1C(=O)OCC)NC(=O)c1ccccc1)C(=O)OC</smiles>

Methyl ((S)-2-((tert-butoxycarbonyl)amino)-3-(2-(2-ethoxy-2oxoethyl)- $1 H$-indol-3-yl)propanoyl)- $L$-alaninate (5a). Prepared according to the general procedure using dipeptide $1 \mathrm{a}(38.9 \mathrm{mg}, 0.1$ mmol), pyridinium salt $4 \mathbf{a}$ (144 mg, $0.3 \mathrm{mmol}$ ), NaOAc ( $16.4 \mathrm{mg}, 0.2$ $\mathrm{mmol})$. The crude mixture was purified by column chromatography 
(hexane/EtOAc gradient from 3:1 to 2:3) to afford the product as a yellow solid (32 $\mathrm{mg}, 68 \%$ yield, average of 3 runs).

${ }^{1} \mathrm{H} \mathrm{NMR}\left(400 \mathrm{MHz}, \mathrm{CDCl}_{3}\right): \delta 8.69(\mathrm{~s}, 1 \mathrm{H}), 7.54(\mathrm{~d}, J=7.8 \mathrm{~Hz}, 1 \mathrm{H}), 7.33-7.26(\mathrm{~m}, 1 \mathrm{H}), 7.13$ (ddd, $J=8.1,7.1,1.3 \mathrm{~Hz}, 1 \mathrm{H}), 7.06(\mathrm{ddd}, J=8.1,7.1,1.1 \mathrm{~Hz}, 1 \mathrm{H}), 6.35(\mathrm{~d}, J=7.0 \mathrm{~Hz}, 1 \mathrm{H}), 5.38$ (brs, $1 \mathrm{H}), 4.46-4.26(\mathrm{~m}, 2 \mathrm{H}), 4.20(\mathrm{q}, J=7.1 \mathrm{~Hz}, 2 \mathrm{H}), 3.91-3.75(\mathrm{~m}, 2 \mathrm{H}), 3.56(\mathrm{~s}, 3 \mathrm{H}), 3.36-$ $3.00(\mathrm{~m}, 2 \mathrm{H}), 1.42(\mathrm{~s}, 9 \mathrm{H}), 1.29(\mathrm{t}, J=7.1 \mathrm{~Hz}, 3 \mathrm{H}), 1.22(\mathrm{~d}, J=7.1 \mathrm{~Hz}, 3 \mathrm{H})$.

${ }^{13} \mathrm{C}$ NMR $\left(101 \mathrm{MHz}, \mathrm{CDCl}_{3}\right): \delta 172.6,171.2,171.1,155.4,137.6,135.8,128.7,128.2,122.1$, $119.8,118.7,110.9,108.4,80.0,61.67,52.4,48.3,31.9,28.4,27.8,18.5,14.3$.

HRMS (ESI) $m / z:[\mathrm{M}+\mathrm{Na}]^{+}$calcd for $\mathrm{C}_{24} \mathrm{H}_{33} \mathrm{~N}_{3} \mathrm{NaO}_{7} 498.2211$; found 498.2212 .<smiles>COC(=O)C([18O])NC(=O)[C@H](Cc1c(CC(=O)[O-])[nH]c2ccccc12)NC(=O)c1ccccc1</smiles>

Methyl ((S)-3-(2-(2-(benzyloxy)-2-oxoethyl)-1H-indol-3-yl)-2((tertbutoxycarbonyl)amino)-propanoyl)-L-alaninate

(5b).

Prepared according to the general procedure using dipeptide 1a (38.9 $\mathrm{mg}, 0.1 \mathrm{mmol})$, pyridinium salt $4 \mathbf{b}$ (163 mg, $0.3 \mathrm{mmol})$, NaOAc (16.4 $\mathrm{mg}, 0.2 \mathrm{mmol})$. The crude mixture was purified by column chromatography (hexane/EtOAc gradient from 3:2) to afford the product as a yellow solid (45 $\mathrm{mg}, 84 \%$ yield, average of two runs).

${ }^{1} \mathrm{H}$ NMR $\left(400 \mathrm{MHz}, \mathrm{CDCl}_{3}\right): \delta 8.62(\mathrm{~s}, 1 \mathrm{H}), 7.55(\mathrm{~d}, J=7.8 \mathrm{~Hz}, 1 \mathrm{H}), 7.40-7.31(\mathrm{~m}, 5 \mathrm{H}), 7.30$ $-7.25(\mathrm{~m}, 1 \mathrm{H}), 7.14(\mathrm{ddd}, J=8.1,7.0,1.2 \mathrm{~Hz}, 1 \mathrm{H}), 7.07(\mathrm{ddd}, J=8.1,7.0,1.1 \mathrm{~Hz}, 1 \mathrm{H}), 6.27(\mathrm{~d}$, $J=7.1 \mathrm{~Hz}, 1 \mathrm{H}), 5.37(\mathrm{~s}, 1 \mathrm{H}), 5.24(\mathrm{~s}, 2 \mathrm{H}), 4.53-4.18(\mathrm{~m}, 2 \mathrm{H}), 4.01-3.78(\mathrm{~m}, 2 \mathrm{H}), 3.54(\mathrm{~s}, 3 \mathrm{H})$, $3.37-2.94(\mathrm{~m}, 2 \mathrm{H}), 1.42(\mathrm{~s}, 9 \mathrm{H}), 1.19(\mathrm{~d}, J=7.1 \mathrm{~Hz}, 3 \mathrm{H})$.

${ }^{13} \mathrm{C}$ NMR $\left(101 \mathrm{MHz}, \mathrm{CDCl}_{3}\right): \delta 172.6,171.1,170.9,155.4,135.9,135.4,128.8,128.6,128.5$, $128.4,128.2,122.2,119.9,118.7,110.9,108.6,80.0,67.4,55.1,52.4,48.2,31.9,28.4,27.9,18.5$.

$\underline{\text { HRMS (ESI) }} m / z:[\mathrm{M}+\mathrm{Na}]^{+}$calcd for $\mathrm{C}_{29} \mathrm{H}_{35} \mathrm{~N}_{3} \mathrm{NaO}_{7} 560.2367$; found 560.2361.<smiles>COC(=O)C(NC(=O)[C@H](Cc1c(CC(=O)O)[nH]c2ccccc12)C(=O)O)[N+](=O)[O-]</smiles>

Methyl ((S)-2-((((9H-fluoren-9-yl)methoxy)carbonyl)amino)-3(2-(2-(benzyloxy)-2-oxoethyl)-1H-indol-3-yl)propanoyl)-Lalaninate $(\mathbf{5 c})$. Prepared according to the general procedure using dipeptide $1 \mathbf{b}$ (51.2 $\mathrm{mg}, 0.1 \mathrm{mmol})$, pyridinium salt $\mathbf{4 b}(163 \mathrm{mg}, 0.3$ $\mathrm{mmol}), \mathrm{NaOAc}(16.4 \mathrm{mg}, 0.2 \mathrm{mmol})$. The crude mixture was purified by column chromatography (hexane/EtOAc gradient from 2:1) to afford the product as a yellow solid (30.1 mg, $46 \%$ yield).

${ }^{1} \mathrm{H} \mathrm{NMR}\left(500 \mathrm{MHz}, \mathrm{CDCl}_{3}\right) \delta 8.48(\mathrm{~s}, 1 \mathrm{H}), 7.68(\mathrm{~d}, J=7.5 \mathrm{~Hz}, 2 \mathrm{H}), 7.50(\mathrm{~d}, J=7.6 \mathrm{~Hz}, 3 \mathrm{H})$, $7.35-7.19(\mathrm{~m}, 11 \mathrm{H}), 7.09-7.04(\mathrm{~m}, 1 \mathrm{H}), 7.00(\mathrm{t}, J=7.2 \mathrm{~Hz}, 1 \mathrm{H}), 6.14(\mathrm{~d}, J=6.9 \mathrm{~Hz}, 1 \mathrm{H}), 5.74$ $(\mathrm{d}, J=7.6 \mathrm{~Hz}, 1 \mathrm{H}), 5.10(\mathrm{~d}, J=2.9 \mathrm{~Hz}, 2 \mathrm{H}), 4.39(\mathrm{~d}, J=7.8 \mathrm{~Hz}, 1 \mathrm{H}), 4.30(\mathrm{t}, J=7.9 \mathrm{~Hz}, 2 \mathrm{H})$, $4.18(\mathrm{t}, J=7.1 \mathrm{~Hz}, 1 \mathrm{H}), 4.11(\mathrm{~s}, 1 \mathrm{H}), 3.92-3.71(\mathrm{~m}, 2 \mathrm{H}), 3.44(\mathrm{~s}, 3 \mathrm{H}), 3.22(\mathrm{~d}, J=14.3 \mathrm{~Hz}, 1 \mathrm{H})$, $3.02(\mathrm{dd}, J=14.4,9.0 \mathrm{~Hz}, 1 \mathrm{H}), 1.12(\mathrm{~d}, J=7.2 \mathrm{~Hz}, 3 \mathrm{H})$.

${ }^{13} \mathrm{C}$ NMR $\left(126 \mathrm{MHz}, \mathrm{CDCl}_{3}\right) \delta 172.3,170.6,155.9,143.9,143.8,141.3,135.7,135.3,128.6$, $128.5,128.4,128.3,128.0,127.7,127.1,127.1,125.2,125.1,122.2,119.96,119.94,119.91$, $118.4,110.8,108.4,67.4,67.1,55.4,52.2,48.2,47.2,31.8,28.0,18.3$.

HRMS (ESI) $m / z:[\mathrm{M}+\mathrm{Na}]^{+}$calcd for $\mathrm{C}_{39} \mathrm{H}_{37} \mathrm{~N}_{3} \mathrm{NaO}_{7}$ 682.2524; found 682.2522 . 


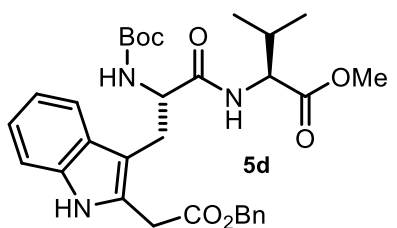

Methyl ((S)-3-(2-(2-(benzyloxy)-2-oxoethyl)-1H-indol-3-yl)-2((tertbutoxycarbonyl)amino)-propanoyl)-L-valinate

(5d).

Prepared according to the general procedure using dipeptide 1c (41.8 $\mathrm{mg}, 0.1 \mathrm{mmol})$, pyridinium salt $4 \mathbf{b}$ (163 mg, $0.3 \mathrm{mmol})$, NaOAc (16.4 $\mathrm{mg}, 0.2 \mathrm{mmol})$. The crude mixture was purified by column chromatography (hexane/EtOAc gradient from 2:1) to afford the product as a yellow solid (40.1 $\mathrm{mg}, 71 \%$ yield).

${ }^{1} \mathrm{H}$ NMR $\left(500 \mathrm{MHz}, \mathrm{CDCl}_{3}\right) \delta 8.61(\mathrm{~s}, 1 \mathrm{H}), 7.54(\mathrm{dd}, J=7.9,1.1 \mathrm{~Hz}, 1 \mathrm{H}), 7.36-7.31(\mathrm{~m}, 5 \mathrm{H})$, $7.28-7.23(\mathrm{~m}, 1 \mathrm{H}), 7.12(\mathrm{ddd}, J=8.2,7.1,1.2 \mathrm{~Hz}, 1 \mathrm{H}), 7.06(\mathrm{ddd}, J=8.0,7.0,1.1 \mathrm{~Hz}, 1 \mathrm{H}), 6.26$ $(\mathrm{d}, J=8.4 \mathrm{~Hz}, 1 \mathrm{H}), 5.38(\mathrm{~s}, 1 \mathrm{H}), 5.17(\mathrm{~d}, J=2.0 \mathrm{~Hz}, 2 \mathrm{H}), 4.42(\mathrm{~d}, J=8.2 \mathrm{~Hz}, 1 \mathrm{H}), 4.25(\mathrm{dd}, J=$ 8.4, $5.1 \mathrm{~Hz}, 1 \mathrm{H}), 3.97-3.79(\mathrm{~m}, 2 \mathrm{H}), 3.48(\mathrm{~s}, 3 \mathrm{H}), 3.22(\mathrm{~d}, J=13.7 \mathrm{~Hz}, 1 \mathrm{H}), 3.10(\mathrm{~d}, J=9.2 \mathrm{~Hz}$, $1 \mathrm{H}), 1.97-1.88(\mathrm{~m}, 1 \mathrm{H}), 1.41(\mathrm{~s}, 9 \mathrm{H}), 0.75(\mathrm{~d}, J=6.9 \mathrm{~Hz}, 3 \mathrm{H}), 0.72(\mathrm{~d}, J=6.9 \mathrm{~Hz}, 3 \mathrm{H})$.

${ }^{13} \mathrm{C}$ NMR $\left(126 \mathrm{MHz}, \mathrm{CDCl}_{3}\right) \delta 171.4,171.3,170.8,155.3,135.7,135.3,128.6,128.5,128.3$, 128.2, 128.0, 122.0, 119.8, 118.5, 110.7, 108.5, 79.9, 67.2, 57.2, 55.1, 55.1, 51.8, 31.8, 31.4, 28.3, 27.6, 18.6, 17.7 .

HRMS (ESI) $m / z:[\mathrm{M}+\mathrm{Na}]^{+}$calcd for $\mathrm{C}_{31} \mathrm{H}_{39} \mathrm{~N}_{3} \mathrm{NaO}_{7}$ 588.2680; found 588.2675.

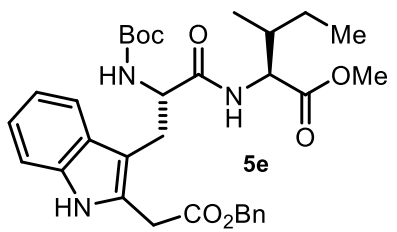

Methyl ((S)-3-(2-(2-(benzyloxy)-2-oxoethyl)-1H-indol-3-yl)-2((tertbutoxycarbonyl)amino)-propanoyl)-L-alloisoleucinate (5e). Prepared according to the general procedure using dipeptide 1d (43.2 $\mathrm{mg}, 0.1 \mathrm{mmol})$, pyridinium salt $4 \mathbf{b}$ (163 mg, $0.3 \mathrm{mmol})$, NaOAc (16.4 $\mathrm{mg}, 0.2 \mathrm{mmol})$. The crude mixture was purified by column chromatography (hexane/EtOAc gradient from 3:1) to afford the product as a yellow solid (36.2 $\mathrm{mg}, 62 \%$ yield).

${ }^{1} \mathrm{H} \mathrm{NMR}\left(400 \mathrm{MHz}, \mathrm{CDCl}_{3}\right) \delta 8.54(\mathrm{~s}, 1 \mathrm{H}), 7.48(\mathrm{~d}, J=7.8 \mathrm{~Hz}, 1 \mathrm{H}), 7.29(\mathrm{~d}, J=2.1 \mathrm{~Hz}, 5 \mathrm{H})$, $7.22-7.19(\mathrm{~m}, 1 \mathrm{H}), 7.06$ (ddd, $J=8.1,7.1,1.3 \mathrm{~Hz}, 1 \mathrm{H}), 7.00(\mathrm{ddd}, J=8.0,7.1,1.1 \mathrm{~Hz}, 1 \mathrm{H}), 6.19$ $(\mathrm{d}, J=8.2 \mathrm{~Hz}, 1 \mathrm{H}), 5.30(\mathrm{~s}, 1 \mathrm{H}), 5.11(\mathrm{~d}, J=1.3 \mathrm{~Hz}, 2 \mathrm{H}), 4.35(\mathrm{~d}, J=7.2 \mathrm{~Hz}, 1 \mathrm{H}), 4.23(\mathrm{dd}, J=$ 8.2, $5.1 \mathrm{~Hz}, 1 \mathrm{H}), 3.82(\mathrm{q}, J=16.9 \mathrm{~Hz}, 2 \mathrm{H}), 3.42(\mathrm{~s}, 3 \mathrm{H}), 3.15(\mathrm{~s}, 1 \mathrm{H}), 3.01(\mathrm{dd}, J=14.4,8.8 \mathrm{~Hz}$, $1 \mathrm{H}), 1.60(\mathrm{dd}, J=4.6,2.3 \mathrm{~Hz}, 1 \mathrm{H}), 1.35(\mathrm{~s}, 9 \mathrm{H}), 1.22-1.13(\mathrm{~m}, 1 \mathrm{H}), 0.95-0.86(\mathrm{~m}, 1 \mathrm{H}), 0.73$ $(\mathrm{t}, J=7.4 \mathrm{~Hz}, 3 \mathrm{H}), 0.63(\mathrm{~d}, J=6.9 \mathrm{~Hz}, 3 \mathrm{H})$.

${ }^{13} \mathrm{C}$ NMR $\left(101 \mathrm{MHz}, \mathrm{CDCl}_{3}\right) \delta 171.2,171.2,170.8,155.3,135.7,135.3,128.6,128.5,128.3$, 128.2, 128.0, 122.0, 119.7, 118.5, 110.7, 108.4, 79.8, 67.2, 56.4, 55.0, 51.8, 38.0, 31.8, 28.3, 27.6, $25.0,15.0,11.4$.

HRMS (ESI) $m / z:[\mathrm{M}+\mathrm{Na}]^{+}$calcd for $\mathrm{C}_{32} \mathrm{H}_{41} \mathrm{~N}_{3} \mathrm{NaO}_{7} 602.2837$; found 602.2839.

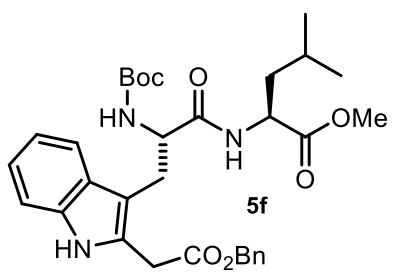

Methyl ((S)-3-(2-(2-(benzyloxy)-2-oxoethyl)-1H-indol-3-yl)-2((tertbutoxycarbonyl)amino)-propanoyl)-L-leucinate

$(\mathbf{5 f})$.

Prepared according to the general procedure using dipeptide 1e (43.2 $\mathrm{mg}, 0.1 \mathrm{mmol}$ ), pyridinium salt $4 \mathbf{b}$ (163 mg, $0.3 \mathrm{mmol})$, NaOAc (16.4 $\mathrm{mg}, 0.2 \mathrm{mmol})$. The crude mixture was purified by column chromatography (hexane/EtOAc gradient from 3:1) to afford the product as a yellow solid ( $34.1 \mathrm{mg}, 59 \%$ yield).

${ }^{1} \mathrm{H} \mathrm{NMR}\left(400 \mathrm{MHz}, \mathrm{CDCl}_{3}\right) \delta 8.57(\mathrm{~s}, 1 \mathrm{H}), 7.50(\mathrm{~d}, J=7.8 \mathrm{~Hz}, 1 \mathrm{H}), 7.28(\mathrm{~d}, J=3.3 \mathrm{~Hz}, 5 \mathrm{H})$, $7.20(\mathrm{~d}, J=7.9 \mathrm{~Hz}, 1 \mathrm{H}), 7.10-7.03(\mathrm{~m}, 1 \mathrm{H}), 7.00(\mathrm{td}, J=7.5,7.0,1.1 \mathrm{~Hz}, 1 \mathrm{H}), 6.09(\mathrm{~d}, J=8.0$ 
$\mathrm{Hz}, 1 \mathrm{H}), 5.28(\mathrm{~d}, J=7.5 \mathrm{~Hz}, 1 \mathrm{H}), 5.11(\mathrm{~d}, J=1.5 \mathrm{~Hz}, 2 \mathrm{H}), 4.42-4.24(\mathrm{~m}, 2 \mathrm{H}), 3.89-3.74(\mathrm{~m}$, $2 \mathrm{H}), 3.44(\mathrm{~s}, 3 \mathrm{H}), 3.20-3.10(\mathrm{~m}, 1 \mathrm{H}), 3.08-2.95(\mathrm{~m}, 1 \mathrm{H}), 1.41-1.29(\mathrm{~m}, 11 \mathrm{H}), 0.78-0.69(\mathrm{~m}$, $6 \mathrm{H})$.

${ }^{13} \mathrm{C}$ NMR $\left(101 \mathrm{MHz}, \mathrm{CDCl}_{3}\right) \delta 172.4,171.2,170.8,155.3,135.7,135.3,128.6,128.5,128.3$, 128.3, 128.0, 122.0, 119.7, 118.5, 110.7, 108.4, 79.9, 67.2, 54.9, 52.0, 50.7, 41.7, 31.8, 28.2, 27.5, 24.5, 22.6, 21.9.

HRMS (ESI) $m / z:[\mathrm{M}+\mathrm{Na}]^{+}$calcd for $\mathrm{C}_{32} \mathrm{H}_{41} \mathrm{~N}_{3} \mathrm{NaO}_{7} 602.2837$; found 602.2837.<smiles>COC(=O)C(Cc1ccccc1)NC(=O)[C@H](Cc1c(CC(=O)O)[nH]c2ccccc12)NC(=O)c1ccccc1</smiles>

${ }^{1} \mathrm{H} \mathrm{NMR}\left(500 \mathrm{MHz}, \mathrm{CDCl}_{3}\right) \delta 8.57(\mathrm{~s}, 1 \mathrm{H}), 7.49(\mathrm{~d}, J=7.8 \mathrm{~Hz}, 1 \mathrm{H}), 7.27(\mathrm{~d}, J=3.0 \mathrm{~Hz}, 4 \mathrm{H})$, $7.21(\mathrm{~d}, J=8.1 \mathrm{~Hz}, 1 \mathrm{H}), 7.10-6.98(\mathrm{~m}, 5 \mathrm{H}), 6.71(\mathrm{~d}, J=7.2 \mathrm{~Hz}, 2 \mathrm{H}), 6.10(\mathrm{~d}, J=7.2 \mathrm{~Hz}, 1 \mathrm{H})$, $5.23(\mathrm{~s}, 1 \mathrm{H}), 5.12-5.01(\mathrm{~m}, 2 \mathrm{H}), 4.41(\mathrm{~d}, J=66.4 \mathrm{~Hz}, 2 \mathrm{H}), 3.78(\mathrm{~d}, J=16.3 \mathrm{~Hz}, 2 \mathrm{H}), 3.42(\mathrm{~s}$, $3 \mathrm{H}), 3.17(\mathrm{~s}, 1 \mathrm{H}), 2.98(\mathrm{dd}, J=14.8,8.3 \mathrm{~Hz}, 1 \mathrm{H}), 2.89-2.67(\mathrm{~m}, 2 \mathrm{H}), 1.34(\mathrm{~s}, 9 \mathrm{H})$.

${ }^{13} \mathrm{C} \mathrm{NMR}\left(126 \mathrm{MHz}, \mathrm{CDCl}_{3}\right) \delta 171.03,170.95,170.70,155.2,135.7,135.6,135.3,129.1,128.6$, $128.5,128.4,128.0,126.9,122.1,119.8,118.6,110.8,108.3,79.9,67.2,54.9,53.3,52.0,37.9$, 31.7, 28.3, 27.6.

HRMS (ESI) $m / z:[\mathrm{M}+\mathrm{Na}]^{+}$calcd for $\mathrm{C}_{35} \mathrm{H}_{39} \mathrm{~N}_{3} \mathrm{NaO}_{7}$ 636.2680; found 636.2666.

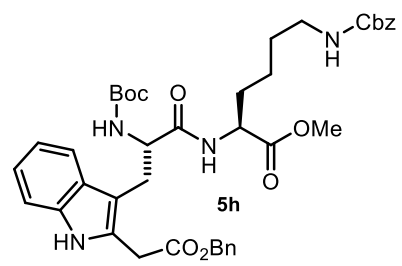

Methyl $\mathrm{N}^{2}$-((S)-3-(2-(2-(benzyloxy)-2-oxoethyl)-1H-indol-3-yl)-2((tertbutoxycarbonyl)-amino)propanoyl)- $\mathrm{N}^{6}$ ((benzyloxy)carbonyl)-L-lysinate (5h). Prepared according to the general procedure using dipeptide $1 \mathrm{~g}(58.1 \mathrm{mg}, 0.1 \mathrm{mmol})$, pyridinium salt $4 \mathbf{b}$ (163 mg, $0.3 \mathrm{mmol}), \mathrm{NaOAc}(16.4 \mathrm{mg}, 0.2 \mathrm{mmol})$. The crude mixture was purified by column chromatography (MeOH/DCM, 1:20) to afford the product as a yellow solid (44.0 $\mathrm{mg}, 60 \%$ yield).

${ }^{1} \mathrm{H}$ NMR $\left(500 \mathrm{MHz}, \mathrm{CDCl}_{3}\right) \delta 8.79(\mathrm{~s}, 1 \mathrm{H}), 7.55(\mathrm{~d}, J=7.8 \mathrm{~Hz}, 1 \mathrm{H}), 7.35-7.29(\mathrm{~m}, 9 \mathrm{H}), 7.26-$ $7.22(\mathrm{~m}, 1 \mathrm{H}), 7.11(\mathrm{t}, J=7.5 \mathrm{~Hz}, 1 \mathrm{H}), 7.08-7.02(\mathrm{~m}, 1 \mathrm{H}), 6.31(\mathrm{~d}, J=7.6 \mathrm{~Hz}, 1 \mathrm{H}), 5.40(\mathrm{~s}, 1 \mathrm{H})$, $5.16(\mathrm{~s}, 2 \mathrm{H}), 5.07(\mathrm{q}, J=12.2 \mathrm{~Hz}, 2 \mathrm{H}), 4.91(\mathrm{~s}, 1 \mathrm{H}), 4.42(\mathrm{~d}, J=7.7 \mathrm{~Hz}, 1 \mathrm{H}), 4.29(\mathrm{~d}, J=6.3 \mathrm{~Hz}$, $1 \mathrm{H}), 3.87(\mathrm{~s}, 2 \mathrm{H}), 3.52(\mathrm{~s}, 3 \mathrm{H}), 3.27(\mathrm{~d}, J=14.0 \mathrm{~Hz}, 1 \mathrm{H}), 3.11-2.96(\mathrm{~m}, 3 \mathrm{H}), 1.90(\mathrm{~s}, 1 \mathrm{H}), 1.65$ $-1.55(\mathrm{~m}, 1 \mathrm{H}), 1.41(\mathrm{~s}, 9 \mathrm{H}), 1.32(\mathrm{q}, J=7.3,6.8 \mathrm{~Hz}, 2 \mathrm{H}), 1.25(\mathrm{~s}, 1 \mathrm{H}), 0.98(\mathrm{p}, J=7.8 \mathrm{~Hz}, 2 \mathrm{H})$.

${ }^{13} \mathrm{C} \mathrm{NMR}\left(126 \mathrm{MHz}, \mathrm{CDCl}_{3}\right) \delta 171.7,171.3,170.8,156.4,155.3,136.6,135.7,135.3,128.6$, $128.5,128.4,128.4,128.3,128.1,128.1,128.0,122.0,119.7,118.5,110.8,108.3,80.0,67.2$, 66.6, 55.1, 52.1, 51.9, 40.6, 31.9, 31.8, 29.2, 28.3, 27.4, 21.8.

HRMS (ESI) $m / z:[\mathrm{M}+\mathrm{Na}]^{+}$calcd for $\mathrm{C}_{40} \mathrm{H}_{48} \mathrm{~N}_{4} \mathrm{NaO}_{9}$ 751.3313; found 751.3307. 
<smiles>COC(=O)C[C@H](NC(=O)[C@H](Cc1c(CC(=O)OCc2ccccc2)[nH]c2ccccc12)NC(=O)OCc1ccccc1)C(=O)O</smiles>

yellow solid (29.8 $\mathrm{mg}, 49 \%$ yield).

${ }^{1} \mathrm{H} \mathrm{NMR}\left(400 \mathrm{MHz}, \mathrm{CDCl}_{3}\right) \delta 8.61(\mathrm{~s}, 1 \mathrm{H}), 7.48(\mathrm{~d}, J=7.8 \mathrm{~Hz}, 1 \mathrm{H}), 7.28(\mathrm{~d}, J=3.2 \mathrm{~Hz}, 5 \mathrm{H})$, $7.20(\mathrm{~s}, 1 \mathrm{H}), 7.06(\mathrm{ddd}, J=8.1,7.1,1.3 \mathrm{~Hz}, 1 \mathrm{H}), 6.99(\mathrm{ddd}, J=8.0,7.0,1.1 \mathrm{~Hz}, 1 \mathrm{H}), 6.61(\mathrm{~d}, J$ $=7.5 \mathrm{~Hz}, 1 \mathrm{H}), 5.22(\mathrm{~s}, 1 \mathrm{H}), 5.10(\mathrm{~s}, 2 \mathrm{H}), 4.48(\mathrm{dt}, J=7.5,4.7 \mathrm{~Hz}, 1 \mathrm{H}), 4.36(\mathrm{~s}, 1 \mathrm{H}), 3.87-3.73$ $(\mathrm{m}, 2 \mathrm{H}), 3.49(\mathrm{~d}, J=5.9 \mathrm{~Hz}, 6 \mathrm{H}), 3.17(\mathrm{~s}, 1 \mathrm{H}), 3.05(\mathrm{dd}, J=14.4,7.9 \mathrm{~Hz}, 1 \mathrm{H}), 2.78-2.67$ (m, $1 \mathrm{H}), 2.63(\mathrm{dd}, J=17.0,5.0 \mathrm{~Hz}, 1 \mathrm{H}), 1.33(\mathrm{~s}, 9 \mathrm{H})$.

${ }^{13} \mathrm{C} \mathrm{NMR}\left(101 \mathrm{MHz}, \mathrm{CDCl}_{3}\right) \delta 171.4,170.9,170.6,170.3,155.2,135.7,135.3,128.6,128.5$, 128.4, 128.3, 128.1, 127.1, 122.0, 119.7, 118.5, 110.7, 108.1, 79.9, 67.2, 54.9, 52.6, 51.8, 48.7, $36.0,31.7,28.2,27.5$.

HRMS (ESI) $m / z:[\mathrm{M}+\mathrm{Na}]^{+}$calcd for $\mathrm{C}_{31} \mathrm{H}_{37} \mathrm{~N}_{3} \mathrm{NaO}_{9} 618.2422$; found 618.2417.

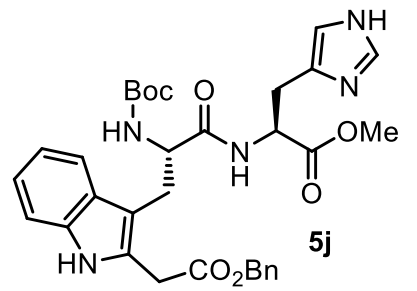

Methyl ((S)-3-(2-(2-(benzyloxy)-2-oxoethyl)-1H-indol-3-yl)-2((tert-butoxycarbonyl)amino)propanoyl)-L-histidinate

(5j). Prepared according to the general procedure using dipeptide $\mathbf{1 i}$ (44.7 mg, $0.1 \mathrm{mmol}$ ), pyridinium salt $4 \mathbf{b}$ (163 mg, $0.3 \mathrm{mmol}$ ), NaOAc (16.4 $\mathrm{mg}, 0.2 \mathrm{mmol}$ ). The crude mixture was purified by column chromatography two consecutive times (MeOH/DCM 1:20 the first time and $\mathrm{MeOH} / \mathrm{Toluene}$ gradient from $5 \%$ to $10 \% \mathrm{MeOH}$ the second) to afford the title compound (11.5 mg, $19.5 \mu \mathrm{mol}, 20 \%)$ as a yellow solid.

${ }^{1} \mathrm{H} \mathrm{NMR}\left(400 \mathrm{MHz} \mathrm{CDCl}_{3}\right) \delta 8.74(\mathrm{~s}, 1 \mathrm{H}), 7.56(\mathrm{~d}, J=7.8 \mathrm{~Hz}, 1 \mathrm{H}), 7.35(\mathrm{~s}, 6 \mathrm{H}), 7.28(\mathrm{~d}, J=8.1$ $\mathrm{Hz}, 1 \mathrm{H}), 7.18-7.05(\mathrm{~m}, 2 \mathrm{H}), 6.65(\mathrm{~s}, 1 \mathrm{H}), 6.56(\mathrm{~s}, 1 \mathrm{H}), 5.50-5.33(\mathrm{~m}, 1 \mathrm{H}), 5.16(\mathrm{~s}, 2 \mathrm{H}), 4.53$ $(\mathrm{s}, 1 \mathrm{H}), 4.42-4.23(\mathrm{~m}, 1 \mathrm{H}), 3.94-3.76(\mathrm{~m}, 2 \mathrm{H}), 3.57(\mathrm{~s}, 3 \mathrm{H}), 3.28-3.03(\mathrm{~m}, 3 \mathrm{H}), 2.98(\mathrm{dd}, J=$ $14.9,4.6 \mathrm{~Hz}, 1 \mathrm{H}), 1.41(\mathrm{~s}, 9 \mathrm{H})$.

${ }^{13} \mathrm{C} \mathrm{NMR}\left(101 \mathrm{MHz}, \mathrm{CDCl}_{3}\right) \delta 171.7,170.8,135.9,135.4,129.2,128.8,128.7,128.5,128.4$, $128.0,125.4,122.3,120.0,118.7,111.0,108.1,80.7,77.5,77.4,77.2,76.8,67.4,55.8,52.9,52.5$, $31.9,29.8,28.5$.

$\underline{\mathrm{HRMS}(\mathrm{ESI})} \mathrm{m} / z:[\mathrm{M}+\mathrm{H}]^{+}$calcd for $\mathrm{C}_{32} \mathrm{H}_{38} \mathrm{~N}_{5} \mathrm{O}_{7}$ 604.2766; found 604.2744.

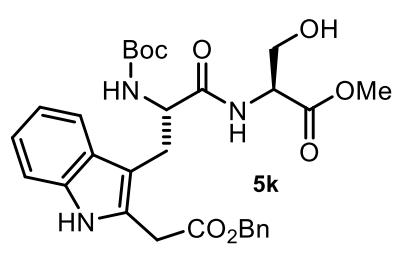

Methyl ((S)-3-(2-(2-(benzyloxy)-2-oxoethyl)-1H-indol-3-yl)-2((tertbutoxycarbonyl)amino)-propanoyl)-L-serinate

$(5 \mathrm{k})$.

Prepared according to the general procedure using dipeptide $\mathbf{1 j}$ (40.5 $\mathrm{mg}, 0.1 \mathrm{mmol}$ ), pyridinium salt $4 \mathbf{b}$ (163 mg, $0.3 \mathrm{mmol})$, NaOAc (16.4 $\mathrm{mg}, 0.2 \mathrm{mmol})$. The crude mixture was purified by column chromatography (hexane/EtOAc, 1:1) to afford the product as a yellow solid (36.1 mg, 65\% yield).

${ }^{1} \mathrm{H} \mathrm{NMR}\left(500 \mathrm{MHz}, \mathrm{CDCl}_{3}\right) \delta 8.56(\mathrm{~s}, 1 \mathrm{H}), 7.46(\mathrm{~d}, J=7.8 \mathrm{~Hz}, 1 \mathrm{H}), 7.29(\mathrm{~d}, J=3.4 \mathrm{~Hz}, 5 \mathrm{H})$, $7.19(\mathrm{~d}, J=7.4 \mathrm{~Hz}, 1 \mathrm{H}), 7.11-7.04(\mathrm{~m}, 1 \mathrm{H}), 7.03-6.97(\mathrm{~m}, 1 \mathrm{H}), 6.57(\mathrm{~d}, J=6.7 \mathrm{~Hz}, 1 \mathrm{H}), 5.32$ $(\mathrm{d}, J=7.5 \mathrm{~Hz}, 1 \mathrm{H}), 5.11(\mathrm{~d}, J=2.0 \mathrm{~Hz}, 2 \mathrm{H}), 4.33(\mathrm{q}, J=7.5 \mathrm{~Hz}, 1 \mathrm{H}), 4.23(\mathrm{~s}, 1 \mathrm{H}), 3.86(\mathrm{~d}, J=$ 
$16.9 \mathrm{~Hz}, 1 \mathrm{H}), 3.81-3.68(\mathrm{~m}, 2 \mathrm{H}), 3.67-3.58(\mathrm{~m}, 1 \mathrm{H}), 3.52(\mathrm{~s}, 3 \mathrm{H}), 3.19(\mathrm{~d}, J=11.5 \mathrm{~Hz}, 1 \mathrm{H})$, 3.05 (dd, $J=14.4,8.1 \mathrm{~Hz}, 1 \mathrm{H}), 2.03(\mathrm{~s}, 1 \mathrm{H}), 1.33$ (s, 9H).

${ }^{13} \mathrm{C}$ NMR $\left(126 \mathrm{MHz}, \mathrm{CDCl}_{3}\right) \delta 172.0,170.8,170.0,155.5,135.7,135.3,128.7,128.5,128.4$, $128.3,128.0,122.2,119.78,118.4,110.8,108.3,80.3,67.4,62.8,55.1,52.5,31.8,28.3$.

$\underline{\text { HRMS (ESI) }} m / z:[\mathrm{M}+\mathrm{Na}]^{+}$calcd for $\mathrm{C}_{29} \mathrm{H}_{35} \mathrm{~N}_{3} \mathrm{NaO}_{8}$ 576.2316; found 576.2320.

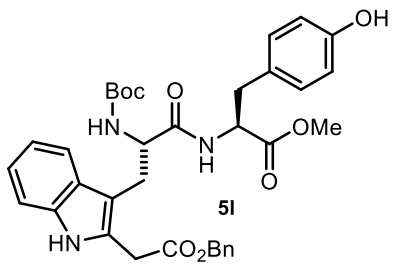

Methyl ((S)-3-(2-(2-(benzyloxy)-2-oxoethyl)-1H-indol-3-yl)-2((tertbutoxycarbonyl)amino)-propanoyl)-L-tyrosinate (5l). Prepared according to the general procedure using dipeptide $\mathbf{1 k}(48.2$ $\mathrm{mg}, 0.1 \mathrm{mmol})$, pyridinium salt $4 \mathbf{b}$ (163 mg, $0.3 \mathrm{mmol})$, NaOAc (16.4 $\mathrm{mg}, 0.2 \mathrm{mmol})$. The crude mixture was purified by column chromatography (hexane/EtOAc gradient from 3:2) to afford the product as a yellow solid ( $38.5 \mathrm{mg}, 61 \%$ yield).

${ }^{1} \mathrm{H} \mathrm{NMR}\left(500 \mathrm{MHz}, \mathrm{CDCl}_{3}\right) \delta 8.58(\mathrm{~s}, 1 \mathrm{H}), 7.53(\mathrm{~d}, J=7.8 \mathrm{~Hz}, 1 \mathrm{H}), 7.32(\mathrm{~d}, J=1.6 \mathrm{~Hz}, 6 \mathrm{H})$, $7.22(\mathrm{~d}, J=8.1 \mathrm{~Hz}, 1 \mathrm{H}), 7.14-7.09(\mathrm{~m}, 1 \mathrm{H}), 7.07-7.00(\mathrm{~m}, 1 \mathrm{H}), 6.62(\mathrm{~d}, J=7.9 \mathrm{~Hz}, 2 \mathrm{H}), 6.55$ $(\mathrm{d}, J=7.6 \mathrm{~Hz}, 2 \mathrm{H}), 6.31(\mathrm{~s}, 1 \mathrm{H}), 5.36(\mathrm{~s}, 1 \mathrm{H}), 5.10(\mathrm{~d}, J=2.9 \mathrm{~Hz}, 2 \mathrm{H}), 4.49(\mathrm{~d}, J=68.8 \mathrm{~Hz}, 2 \mathrm{H})$, $3.83-3.64(\mathrm{~m}, 2 \mathrm{H}), 3.51(\mathrm{~s}, 3 \mathrm{H}), 3.17-2.93(\mathrm{~m}, 2 \mathrm{H}), 2.84(\mathrm{~s}, 1 \mathrm{H}), 2.70(\mathrm{dd}, J=13.9,6.6 \mathrm{~Hz}$, $1 \mathrm{H}), 1.90(\mathrm{dd}, J=12.6,3.9 \mathrm{~Hz}, 1 \mathrm{H}), 1.71-1.53(\mathrm{~m}, 1 \mathrm{H}), 1.37(\mathrm{~s}, 9 \mathrm{H})$.

${ }^{13} \mathrm{C} \mathrm{NMR}\left(126 \mathrm{MHz}, \mathrm{CDCl}_{3}\right) \delta 171.4,171.2,170.8,155.3,135.7,135.4,130.2,128.6,128.5$, $128.4,128.4,127.0,122.1,119.8,118.6,115.4,110.8,108.1,80.1,67.2,55.0,53.4,52.1,49.5$, $37.2,33.7,31.7,28.2,27.5,25.5,24.8$.

$\underline{\text { HRMS (ESI) }} m / z:[\mathrm{M}+\mathrm{Na}]^{+}$calcd for $\mathrm{C}_{35} \mathrm{H}_{39} \mathrm{~N}_{3} \mathrm{NaO}_{8}$ 652.2629; found 652.2607.

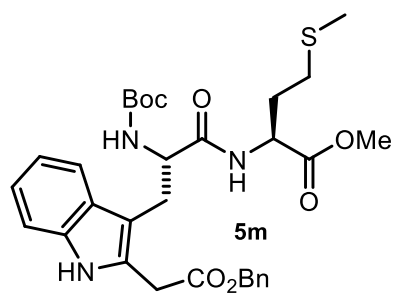

Methyl ((S)-3-(2-(2-(benzyloxy)-2-oxoethyl)-1H-indol-3-yl)-2((tertbutoxycarbonyl)amino)-propanoyl)-L-methioninate $(5 \mathrm{~m})$. Prepared according to the general procedure using dipeptide 11 (45.0 $\mathrm{mg}, 0.1 \mathrm{mmol})$, pyridinium salt $4 \mathbf{b}$ (163 mg, $0.3 \mathrm{mmol})$, NaOAc (16.4 $\mathrm{mg}, 0.2 \mathrm{mmol})$. The crude mixture was purified by column chromatography (hexane/EtOAc gradient from 3:2) to afford the product as a yellow solid (43.1 $\mathrm{mg}, 72 \%$ yield).

${ }^{1} \mathrm{H} \mathrm{NMR}\left(400 \mathrm{MHz}, \mathrm{CDCl}_{3}\right) \delta 8.54(\mathrm{~s}, 1 \mathrm{H}), 7.47(\mathrm{~d}, J=7.8 \mathrm{~Hz}, 1 \mathrm{H}), 7.32-7.24(\mathrm{~m}, 5 \mathrm{H}), 7.22-$ $7.18(\mathrm{~m}, 1 \mathrm{H}), 7.12-7.03(\mathrm{~m}, 1 \mathrm{H}), 7.00(\mathrm{ddd}, J=8.1,7.0,1.1 \mathrm{~Hz}, 1 \mathrm{H}), 6.30(\mathrm{~d}, J=7.5 \mathrm{~Hz}, 1 \mathrm{H})$, $5.30(\mathrm{~s}, 1 \mathrm{H}), 5.12(\mathrm{~d}, J=1.3 \mathrm{~Hz}, 2 \mathrm{H}), 4.32(\mathrm{~d}, J=8.5 \mathrm{~Hz}, 2 \mathrm{H}), 3.82(\mathrm{q}, J=16.9 \mathrm{~Hz}, 2 \mathrm{H}), 3.46(\mathrm{~s}$, $3 \mathrm{H}), 3.19(\mathrm{~d}, J=8.7 \mathrm{~Hz}, 1 \mathrm{H}), 3.01(\mathrm{dd}, J=14.3,8.5 \mathrm{~Hz}, 1 \mathrm{H}), 2.23-2.10(\mathrm{~m}, 2 \mathrm{H}), 1.89(\mathrm{~s}, 4 \mathrm{H})$, $1.77-1.63(\mathrm{~m}, 1 \mathrm{H}), 1.36(\mathrm{~s}, 9 \mathrm{H})$.

${ }^{13} \mathrm{C} \mathrm{NMR}\left(101 \mathrm{MHz}, \mathrm{CDCl}_{3}\right) \delta 171.4,171.3,170.7,155.2,135.7,135.3,128.6,128.5,128.34$, $128.27,128.0,122.1,119.8,118.5,110.7,108.4,79.9,67.3,55.1,52.2,51.5,31.8,31.7,29.5$, $28.3,27.6,15.2$.

HRMS (ESI) $m / z:[\mathrm{M}+\mathrm{Na}]^{+}$calcd for $\mathrm{C}_{31} \mathrm{H}_{39} \mathrm{~N}_{3} \mathrm{NaO}_{7} \mathrm{~S}$ 620.2401; found 620.2409. 
<smiles>COC(Cc1c(CC(=O)OC(C)(C)C)[nH]c2ccccc12)NC(=O)[C@H](Cc1c(CC(=O)OCc2ccccc2)[nH]c2ccccc12)NC(=O)c1ccccc1</smiles>

product as a yellow solid ( $36.3 \mathrm{mg}, 45 \%$ yield). $11 \mathrm{H}), 7.13(\mathrm{dd}, J=13.6,8.0 \mathrm{~Hz}, 3 \mathrm{H}), 7.04-7.00(\mathrm{~m}, 1 \mathrm{H}), 6.96(\mathrm{t}, J=7.4 \mathrm{~Hz}, 2 \mathrm{H}), 6.78(\mathrm{~s}, 1 \mathrm{H})$, $6.30(\mathrm{~s}, 1 \mathrm{H}), 5.31-5.14(\mathrm{~m}, 1 \mathrm{H}), 5.03(\mathrm{~d}, J=8.4 \mathrm{~Hz}, 4 \mathrm{H}), 4.59(\mathrm{~d}, J=7.2 \mathrm{~Hz}, 1 \mathrm{H}), 4.34(\mathrm{~s}, 1 \mathrm{H})$, $3.70(\mathrm{~d}, J=5.1 \mathrm{~Hz}, 2 \mathrm{H}), 3.48(\mathrm{~s}, 2 \mathrm{H}), 3.31(\mathrm{~s}, 2 \mathrm{H}), 3.12(\mathrm{~d}, J=14.7 \mathrm{~Hz}, 1 \mathrm{H}), 3.02-2.90(\mathrm{~m}, 3 \mathrm{H})$, $1.28(\mathrm{~s}, 9 \mathrm{H})$.

${ }^{13} \mathrm{C} \mathrm{NMR}\left(126 \mathrm{MHz}, \mathrm{CDCl}_{3}\right) \delta 171.5,171.3,170.7,170.3,155.1,135.6,135.5,135.4,135.3$, 128.64, 128.60, 128.5, 128.43, 128.40, 128.3, 128.1, 128.0, 122.04, 121.97, 119.7, 119.6, 118.7, $118.3,110.8,110.7,108.3,107.5,79.7,67.2,67.1,54.9,52.7,52.2,31.6,31.3,28.2,26.9$.

HRMS (ESI) $m / z:[\mathrm{M}+\mathrm{H}]^{+}$calcd for $\mathrm{C}_{46} \mathrm{H}_{47} \mathrm{~N}_{4} \mathrm{O}_{9} 799.3349$; found 799.3329.

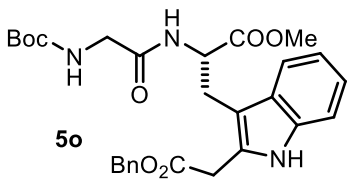

Methyl (S)-3-(2-(2-(benzyloxy)-2-oxoethyl)-1H-indol-3-yl)-2-(2((tertbutoxycarbonyl)-amino)acetamido)propanoate (5o). Prepared according to the general procedure using dipeptide $1 \mathrm{n}(37.5 \mathrm{mg}, 0.1$ mmol), pyridinium salt $4 \mathbf{b}(163 \mathrm{mg}, 0.3 \mathrm{mmol}), \mathrm{NaOAc}(16.4 \mathrm{mg}, 0.2$ $\mathrm{mmol}$ ). The crude mixture was purified by column chromatography (hexane/EtOAc, 3:2) to afford the product as a yellow solid $(30.2 \mathrm{mg}, 58 \%$ yield $)$.

${ }^{1} \mathrm{H} \mathrm{NMR}\left(500 \mathrm{MHz} \mathrm{CDCl}_{3}\right) \delta 8.61(\mathrm{~s}, 1 \mathrm{H}), 7.38(\mathrm{~d}, J=7.8 \mathrm{~Hz}, 1 \mathrm{H}), 7.28(\mathrm{~d}, J=1.2 \mathrm{~Hz}, 5 \mathrm{H})$, $7.20(\mathrm{~d}, J=8.1 \mathrm{~Hz}, 1 \mathrm{H}), 7.07(\mathrm{ddd}, J=8.1,7.0,1.2 \mathrm{~Hz}, 1 \mathrm{H}), 7.01(\mathrm{ddd}, J=8.0,7.0,1.1 \mathrm{~Hz}, 1 \mathrm{H})$, $6.70(\mathrm{~d}, J=7.4 \mathrm{~Hz}, 1 \mathrm{H}), 5.11(\mathrm{~s}, 2 \mathrm{H}), 4.99(\mathrm{~d}, J=5.6 \mathrm{~Hz}, 1 \mathrm{H}), 4.79(\mathrm{dt}, J=7.5,5.9 \mathrm{~Hz}, 1 \mathrm{H}), 3.73$ $(\mathrm{s}, 2 \mathrm{H}), 3.64(\mathrm{~s}, 2 \mathrm{H}), 3.55(\mathrm{~s}, 3 \mathrm{H}), 3.22-3.13(\mathrm{~m}, 2 \mathrm{H}), 1.32(\mathrm{~s}, 9 \mathrm{H})$.

${ }^{13} \mathrm{C} \mathrm{NMR}\left(126 \mathrm{MHz}, \mathrm{CDCl}_{3}\right) \delta 172.1,170.6,169.3,155.8,135.7,135.2,128.8,128.7,128.6$, $128.5,128.3,128.0,122.3,119.8,118.3,111.0,107.7,80.0,67.5,52.8,52.4,43.9,31.8,29.7$, $28.2,26.8$.

$\underline{\operatorname{HRMS}(\mathrm{ESI})} \mathrm{m} / z:[\mathrm{M}+\mathrm{Na}]^{+}$calcd for $\mathrm{C}_{28} \mathrm{H}_{33} \mathrm{~N}_{3} \mathrm{NaO}_{7}$ 546.2211; found 546.2211.

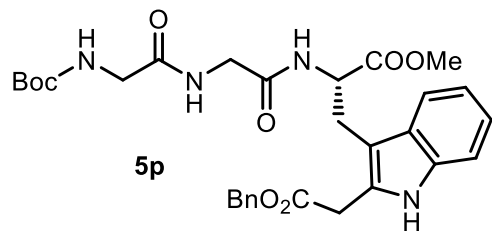

Methyl (S)-12-((2-(2-(benzyloxy)-2-oxoethyl)-1H-indol-3yl)methyl)-2,2-dimethyl-4,7,10- trioxo-3-oxa-5,8,11triazatridecan-13-oate (5p). Prepared according to the general procedure using tripeptide $10(43.3 \mathrm{mg}, 0.1 \mathrm{mmol})$, pyridinium salt $\mathbf{4 b}$ (163 $\mathrm{mg}, 0.3 \mathrm{mmol})$, NaOAc (16.4 mg, 0.2 mmol). The crude mixture was purified by column chromatography (hexane/EtOAc, 1:2 to $1: 4$ ) to afford the product as a yellow solid ( $23.5 \mathrm{mg}, 41 \%$ yield).

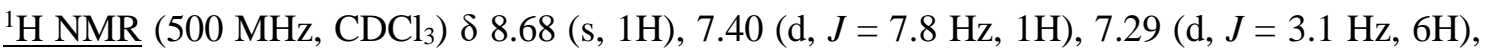
$7.21(\mathrm{~d}, J=8.1 \mathrm{~Hz}, 1 \mathrm{H}), 7.07(\mathrm{ddd}, J=8.2,7.1,1.2 \mathrm{~Hz}, 1 \mathrm{H}), 7.01(\mathrm{ddd}, J=8.0,7.1,1.1 \mathrm{~Hz}, 1 \mathrm{H})$, $6.87(\mathrm{~d}, J=7.4 \mathrm{~Hz}, 1 \mathrm{H}), 6.60(\mathrm{t}, J=5.3 \mathrm{~Hz}, 1 \mathrm{H}), 5.11(\mathrm{~d}, J=2.0 \mathrm{~Hz}, 2 \mathrm{H}), 5.04(\mathrm{~d}, J=11.3 \mathrm{~Hz}$, 
$1 \mathrm{H}), 4.81-4.73(\mathrm{~m}, 1 \mathrm{H}), 3.73(\mathrm{~d}, J=8.2 \mathrm{~Hz}, 4 \mathrm{H}), 3.58(\mathrm{~s}, 5 \mathrm{H}), 3.21(\mathrm{dd}, J=14.9,5.6 \mathrm{~Hz}, 1 \mathrm{H})$, $3.13(\mathrm{dd}, J=14.9,6.9 \mathrm{~Hz}, 1 \mathrm{H}), 1.36(\mathrm{~s}, 9 \mathrm{H})$.

${ }^{13} \mathrm{C}$ NMR $\left(126 \mathrm{MHz}, \mathrm{CDCl}_{3}\right) \delta 172.2,170.8,169.7,168.7,156.0,135.7,135.1,128.7,128.6$, $128.5,128.4,127.9,122.3,119.8,118.3,111.0,107.7,80.3,67.6,52.8,52.5,46.1,44.1,42.2$, $31.8,28.3,26.5,8.6$.

HRMS (ESI) $m / z:[\mathrm{M}+\mathrm{Na}]^{+}$calcd for $\mathrm{C}_{30} \mathrm{H}_{36} \mathrm{~N}_{4} \mathrm{NaO}_{8}$ 603.2425; found 603.2411.

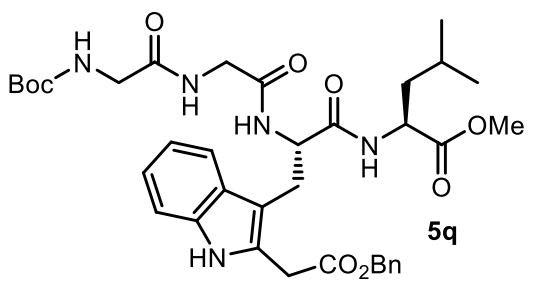

Methyl ((S)-3-(2-(2-(benzyloxy)-2-oxoethyl)-1H-indol-3yl)-2-(2-(2-((tertbutoxycarbonyl)aminoacetamido)acetamido)propanoyl)-L-leucinate (5q). Prepared according to the general procedure using tetrapeptide 1p (54.6 mg, $0.1 \mathrm{mmol})$, pyridinium salt $4 \mathbf{b}$ (163 $\mathrm{mg}, 0.3 \mathrm{mmol}), \mathrm{NaOAc}(16.4 \mathrm{mg}, 0.2 \mathrm{mmol})$. The crude mixture was purified by column chromatography (hexane/EtOAc, 1:2 to 1:4) to afford the product as a yellow solid ( $35.1 \mathrm{mg}, 51 \%$ yield).

$\underline{{ }^{1} \mathrm{H} \mathrm{NMR}}\left(500 \mathrm{MHz}, \mathrm{CDCl}_{3}\right) \delta 8.76(\mathrm{~s}, 1 \mathrm{H}), 7.44(\mathrm{~d}, J=7.8 \mathrm{~Hz}, 1 \mathrm{H}), 7.27(\mathrm{~d}, J=2.1 \mathrm{~Hz}, 5 \mathrm{H})$, $7.15(\mathrm{~d}, J=8.1 \mathrm{~Hz}, 1 \mathrm{H}), 7.10-7.04(\mathrm{~m}, 1 \mathrm{H}), 7.04-6.88(\mathrm{~m}, 4 \mathrm{H}), 5.33(\mathrm{~d}, J=5.9 \mathrm{~Hz}, 1 \mathrm{H}), 5.09$ $(\mathrm{d}, J=1.3 \mathrm{~Hz}, 2 \mathrm{H}), 4.75(\mathrm{q}, J=7.3 \mathrm{~Hz}, 1 \mathrm{H}), 4.37(\mathrm{q}, J=8.1,7.3 \mathrm{~Hz}, 1 \mathrm{H}), 3.82(\mathrm{~d}, J=16.9 \mathrm{~Hz}$, $1 \mathrm{H}), 3.77-3.61(\mathrm{~m}, 5 \mathrm{H}), 3.50(\mathrm{~s}, 3 \mathrm{H}), 3.10(\mathrm{t}, J=6.4 \mathrm{~Hz}, 2 \mathrm{H}), 1.46-1.40(\mathrm{~m}, 2 \mathrm{H}), 1.37(\mathrm{~s}, 10 \mathrm{H})$, $0.76(\mathrm{~d}, J=4.4 \mathrm{~Hz}, 6 \mathrm{H})$.

${ }^{13} \mathrm{C} \mathrm{NMR}\left(126 \mathrm{MHz}, \mathrm{CDCl}_{3}\right) \delta 172.8,171.09,171.06,169.8,168.6,156.1,135.7,135.3,128.6$, $128.5,128.4,127.9,122.0,119.7,118.5,110.9,108.3,80.1,67.4,53.6,52.1,51.0,43.8,42.7$, $41.2,31.8,28.3,27.1,24.7,22.6,22.0$.

HRMS (ESI) $m / z:[\mathrm{M}+\mathrm{Na}]^{+}$calcd for $\mathrm{C}_{36} \mathrm{H}_{47} \mathrm{~N}_{5} \mathrm{NaO}_{9}$ 716.3266; found 716.3261 .

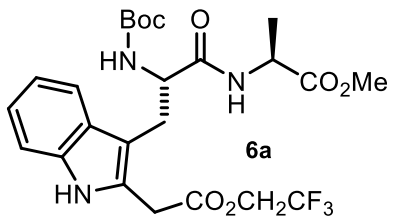

Methyl ((S)-2-((tert-butoxycarbonyl)amino)-3-(2-(2-oxo-2(2,2,2trifluoroethoxy)ethyl)-1H-indol-3-yl)propanoyl)-L-alaninate

(6a). Prepared according to the general procedure using dipeptide 1a (38.9 mg, $0.1 \mathrm{mmol})$, pyridinium salt $4 \mathbf{c}(161 \mathrm{mg}, 0.3 \mathrm{mmol}), \mathrm{NaOAc}$ (16.4 mg, $0.2 \mathrm{mmol})$. The crude mixture was purified by column chromatography (hexane/EtOAc 7:3) to afford the product as a white solid (36.4 $\mathrm{mg}, 69 \%$ yield).

${ }^{1} \mathrm{H} \mathrm{NMR}\left(400 \mathrm{MHz}, \mathrm{CDCl}_{3}\right): \delta 8.49(\mathrm{~s}, 1 \mathrm{H}), 7.57(\mathrm{~d}, J=7.9 \mathrm{~Hz}, 1 \mathrm{H}), 7.31(\mathrm{~d}, J=8.0 \mathrm{~Hz}, 1 \mathrm{H})$, $7.16(\mathrm{t}, J=7.5 \mathrm{~Hz}, 1 \mathrm{H}), 7.09(\mathrm{t}, J=7.5 \mathrm{~Hz}, 1 \mathrm{H}), 6.19(\mathrm{~d}, J=7.1 \mathrm{~Hz}, 1 \mathrm{H}), 5.32(\mathrm{~s}, 1 \mathrm{H}), 4.54(\mathrm{qd}$, $J=8.3,3.2 \mathrm{~Hz}, 2 \mathrm{H}), 4.46-4.34(\mathrm{~m}, 1 \mathrm{H}), 4.34-4.21(\mathrm{~m}, 1 \mathrm{H}), 4.07-3.85(\mathrm{~m}, 2 \mathrm{H}), 3.57(\mathrm{~s}, 3 \mathrm{H})$, $3.36-2.99(\mathrm{~m}, 2 \mathrm{H}), 1.43(\mathrm{~s}, 9 \mathrm{H}), 1.22(\mathrm{~d}, J=7.1 \mathrm{~Hz}, 3 \mathrm{H})$.

${ }^{13} \mathrm{C}$ NMR $\left(101 \mathrm{MHz}, \mathrm{CDCl}_{3}\right): \delta 172.7,171.1,169.6,155.4,136.0,128.2,127.3,126.2,124.0$, $122.5,121.8,120.1,118.8,111.0,109.2,80.2,61.2$ (q), 55.1, 52.4, 48.3, 31.4, 28.4, 27.8, 18.5.

${ }^{19} \mathrm{~F} \mathrm{NMR}\left(376 \mathrm{MHz}, \mathrm{CDCl}_{3}\right): \delta-73.65$.

HRMS (ESI) $m / z:[\mathrm{M}+\mathrm{Na}]^{+}$calcd for $\mathrm{C}_{24} \mathrm{H}_{30} \mathrm{~F}_{3} \mathrm{~N}_{3} \mathrm{NaO}_{7}$ 552.1928; found 552.1925. 
<smiles>CC(=O)C(C)NC(=O)[C@H](Cc1c(C(C)C(C)=O)[nH]c2ccccc12)NC(=O)c1ccccc1</smiles>

Methyl 2-(3-((S)-2-((tert-butoxycarbonyl)amino)-3-(((S)-1 methoxy-1-oxopropan-2-yl)amino)-3-oxopropyl)-1H-indol-2yl)propanoate (6b). Prepared according to the general procedure using dipeptide 1a (38.9 mg, $0.1 \mathrm{mmol})$, pyridinium salt KS-AlaOMe (144 mg, $0.3 \mathrm{mmol}$ ), NaOAc (16.4 mg, $0.2 \mathrm{mmol}$ ). The crude mixture was purified by column chromatography (gradient hexane/EtOAc 3:2 to 1:1) to afford the product as a white solid $(19.8 \mathrm{mg}, 42 \%$ yield, mixture of 2 inseparable diastereoisomers in a 1.2:1 ratio).

${ }^{1} \mathrm{H}$ NMR $\left(400 \mathrm{MHz}, \mathrm{CDCl}_{3}\right): 8.57(\mathrm{~s}, 0.5 \mathrm{H}), 8.37(\mathrm{~s}, 0.5 \mathrm{H}), 8.21-8.15(\mathrm{~m},, 0.5 \mathrm{H}), 7.65-7.46$ $(\mathrm{m}, 1.5 \mathrm{H}), 7.34-7.25(\mathrm{~m}, 1 \mathrm{H}), 7.14(\mathrm{dddd}, 1 \mathrm{H}), 7.10-7.03(\mathrm{~m} 1 \mathrm{H}), 6.32(\mathrm{~d}, J=7.0 \mathrm{~Hz}, 0.5 \mathrm{H})$, $6.25(\mathrm{~d}, J=7.1 \mathrm{~Hz}, 0.5 \mathrm{H}), 5.46-5.18(\mathrm{~s}, 1 \mathrm{H}), 4.53-4.07(\mathrm{~m}, 2 \mathrm{H}), 3.74(\mathrm{~s}, 1.5 \mathrm{H}), 3.72(\mathrm{~s}, 1.5 \mathrm{H})$, $3.61(\mathrm{~s}, 1.5 \mathrm{H}), 3.53(\mathrm{~s}, 1.5 \mathrm{H}), 3.37-3.03(\mathrm{~m}, 2 \mathrm{H}), 1.55(\mathrm{~d}, J=6.9 \mathrm{~Hz}, 3 \mathrm{H}), 1.41(\mathrm{~s}, 9 \mathrm{H}), 1.30-$ $1.20(\mathrm{~d}, J=5.7 \mathrm{~Hz}, 3 \mathrm{H})$

${ }^{13} \mathrm{C} \mathrm{NMR}\left(101 \mathrm{MHz}, \mathrm{CDCl}_{3}\right): 174.4,172.7 \& 172.6,171.2,155.4,155.9 \& 135.8,134.4 \& 134.0$, $129.0,122.3,119.9,119.0 \& 118.8,110.9 \& 110.8,107.5 \& 107.2,80.1,60.5,52.7 \& 52.6,52.5$ $\& 52.4,48.3,48.2,36.8,28.4,18.8,18.1$.

HRMS (ESI) $m / z:$ : $\mathrm{M}-\mathrm{H}]^{-}$calcd for $\mathrm{C}_{24} \mathrm{H}_{32} \mathrm{~N}_{3} \mathrm{O}_{7} 474.2246$; found 474.2241 .<smiles>CC(=O)OC(C)C(=O)NC(Cc1c(C(C)C(=O)OCc2ccccc2)[nH]c2ccccc12)C(=O)OCc1ccccc1</smiles>

Benzyl 2-(3-((S)-2-((tert-butoxycarbonyl)amino)-3-(((S)-1methoxy-1-oxopropan-2-yl)amino)-3-oxopropyl)-1H-indol-2yl)propanoate $(\mathbf{6 c})$. Prepared according to the general procedure using dipeptide 1a (38.9 mg, $0.1 \mathrm{mmol}$ ), pyridinium salt KS-AlaOBn (167 mg, $0.3 \mathrm{mmol}), \mathrm{NaOAc}(16.4 \mathrm{mg}, 0.2 \mathrm{mmol})$. The crude mixture was purified by column chromatography (hexane/EtOAc 7:3) to afford the product as a white solid $(25.5 \mathrm{mg}, 46 \%$ yield, mixture of 2 inseparable diastereoisomers in 1:1 ratio).

${ }^{1} \mathrm{H} \mathrm{NMR}\left(400 \mathrm{MHz}, \mathrm{CDCl}_{3}\right): 8.54(\mathrm{~s}, 0.5 \mathrm{H}), 8.34(\mathrm{~s}, 0.5 \mathrm{H}), 7.56(\mathrm{~d}, 7.8 \mathrm{~Hz}, 0.5 \mathrm{H}), 7.49$ (d, 7.8 $\mathrm{Hz}, 0.5 \mathrm{H}), 7.41-7.23(\mathrm{~m}, 7 \mathrm{H}), 7.18-7.02(\mathrm{~m}, 2 \mathrm{H}), 6.21(\mathrm{~d}, J=7.0 \mathrm{~Hz}, 0.5 \mathrm{H}), 6.12(\mathrm{~d}, J=7.0$ $\mathrm{Hz}, 0.5 \mathrm{H}), 5.48-5.24(\mathrm{~m}, 1 \mathrm{H}), 5.20(\mathrm{~d}, J=12.3 \mathrm{~Hz}, 1 \mathrm{H}), 5.09(\mathrm{dd}, J=12.3,1.8 \mathrm{~Hz}, 1 \mathrm{H}), 3.59$ (s, 1.5H), $3.51(\mathrm{~s}, 1.5 \mathrm{H}), 3.37-3.05(\mathrm{~m}, 2 \mathrm{H}), 1.69(\mathrm{~s}, 2 \mathrm{H}), 1.57$ (d, $J=7.2 \mathrm{~Hz}, 3 \mathrm{H}), 1.49-1.33$ $(\mathrm{m}, 9 \mathrm{H}), 1.22(\mathrm{~d}, J=7.2 \mathrm{~Hz}, 3 \mathrm{H})$.

${ }^{13} \mathrm{C} \mathrm{NMR}\left(126 \mathrm{MHz}, \mathrm{CDCl}_{3}\right): 174.1 \& 173.9,172.7 \& 172.5,171.2 \& 171.1,155.4,135.84 \&$ $135.77,135.64 \& 135.53,134.3 \& 134.0,128.75 \& 128.74,128.54 \& 128.51,128.3,128.29 \&$ $128.19,122.3,119.93,118.92 \& 118.72,110.95 \& 110.82,107.7 \& 107.4,80.2,67.4,67.2,52.45$ $\& 52.32,48.28 \& 48.17,37.1,29.8,28.4,18.61 \& 18.55,18.1$.

HRMS (ESI) $m / z:[\mathrm{M}-\mathrm{H}]^{-}$calcd for $\mathrm{C}_{30} \mathrm{H}_{36} \mathrm{~N}_{3} \mathrm{O}_{7} 550.2559$; found 550.2547 .

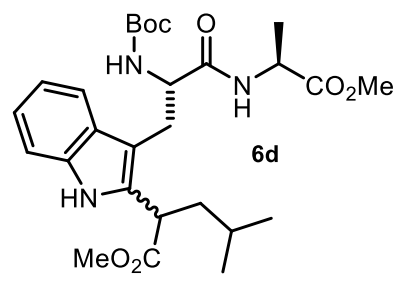

Methyl 2-(3-((S)-2-((tert-butoxycarbonyl)amino)-3-(((S)-1methoxy-1-oxopropan-2-yl)amino)-3-oxopropyl)-1H-indol-2-yl)4-methylpentanoate (6d). Prepared according to the general procedure using dipeptide $1 \mathbf{1 a}(38.9 \mathrm{mg}, 0.1 \mathrm{mmol})$, pyridinium salt KS-Leu-OMe (157 mg, $0.3 \mathrm{mmol})$, NaOAc (16.4 mg, $0.2 \mathrm{mmol})$. The crude mixture was purified by column chromatography (hexane/EtOAc 7:3) to afford the product as a white solid $(16.1 \mathrm{mg}, 30 \%$ yield, mixture of 2 inseparable diastereoisomer in 1:1 ratio). 
${ }^{1} \mathrm{H} \mathrm{NMR}\left(400 \mathrm{MHz}, \mathrm{CDCl}_{3}\right): 8.51(\mathrm{~s}, 0.5 \mathrm{H}), 8.33(\mathrm{~s}, 0.5 \mathrm{H}), 7.66-7.55(\mathrm{~m}, 1 \mathrm{H}), 7.69-7.55(\mathrm{~m}$, $0.5 \mathrm{H}), 7.51(\mathrm{~d}, J=7.9 \mathrm{~Hz}, 0.5 \mathrm{H}), 7.35-7.26(\mathrm{~m}, 1 \mathrm{H}), 7.23-7.00(\mathrm{~m}, 2 \mathrm{H}), 6.33(\mathrm{~d}, J=6.9 \mathrm{~Hz}$, $0.5 \mathrm{H}), 6.20(\mathrm{~d}, J=7.1 \mathrm{~Hz}, 0.5 \mathrm{H}), 5.42-5.10(\mathrm{~m}, 1 \mathrm{H}), 4.52-4.20(\mathrm{~m}, 2 \mathrm{H}), 4.18-3.97(\mathrm{~m}, 1 \mathrm{H})$, $3.74(\mathrm{~s}, 1.5 \mathrm{H}), 3.73(\mathrm{~s}, 1.5 \mathrm{H}), 3.33(\mathrm{dd}, J=14.6,5.7 \mathrm{~Hz}, 0.5 \mathrm{H}), 3.24-2.98(\mathrm{~m}, 1.5 \mathrm{H}), 2.03-$ $1.92(\mathrm{~m}, 1 \mathrm{H}), 1.41(\mathrm{~s}, 9 \mathrm{H}) .1 .33-1.15(\mathrm{~m}, 5 \mathrm{H}), 0.93(\mathrm{~s}, 3 \mathrm{H}), 0.92(\mathrm{~s}, 3 \mathrm{H})$.

${ }^{13} \mathrm{C} \mathrm{NMR}\left(101 \mathrm{MHz}, \mathrm{CDCl}_{3}\right): 174.6 \& 174.2,172.7,171.3,155.4,135.9,133.3,128.1,122.5 \&$ $122.3,119.96 \& 119.93,119.1 \& 118.8,110.93 \& 110.86,108.2 \& 107.8,80.2,55.3,52.4,48.29$ $\& 48.25,42.1,41.00 \& 40.94,29.8 \& 29.6,28.4,26.4 \& 26.2,22.6 \& 22.5,18.8$.

HRMS (ESI) $m / z:[\mathrm{M}-\mathrm{H}]^{-}$calcd for $\mathrm{C}_{27} \mathrm{H}_{38} \mathrm{~N}_{3} \mathrm{O}_{7}$ 516.2715; found 516.2716.

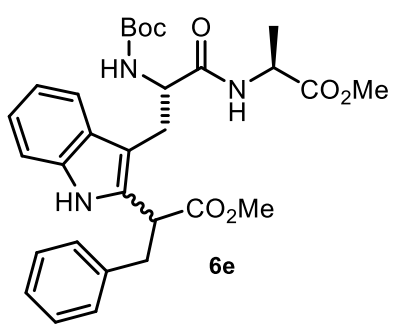

Methyl 2-(3-((S)-2-((tert-butoxycarbonyl)amino)-3-(((S)-1-
methoxy-1-oxopropan-2-yl)amino)-3-oxopropyl)-1H-indol-2-yl)-
3-phenylpropanoate (6e). Prepared according to the general
procedure using dipeptide 1a (38.9 $\mathrm{mg}, 0.1 \mathrm{mmol})$, pyridinium salt
KS-Phe-OMe (167 $\mathrm{mg}, 0.3 \mathrm{mmol}), \mathrm{NaOAc}(16.4 \mathrm{mg}, 0.2 \mathrm{mmol})$. The
crude mixture was purified by column chromatography (gradient of
hexane/EtOAc $3: 1$ to $3: 2)$ to afford the product as a yellow solid (27.8 $\mathrm{mg}, 50 \%$ yield, mixture of 2 inseparable diastereoisomers in 1:1 ratio).

${ }^{1} \mathrm{H} \mathrm{NMR}\left(400 \mathrm{MHz}, \mathrm{CDCl}_{3}\right): \delta 8.73(\mathrm{~s}, 0.5 \mathrm{H}), 8.62(\mathrm{~s}, 0.5 \mathrm{H}), 7.47(\mathrm{~d}, J=8.0 \mathrm{~Hz}, 0.5 \mathrm{H}), 7.47(\mathrm{~d}$, $J=8.0 \mathrm{~Hz}, 0.5 \mathrm{H}), 7.33(\mathrm{t}, J=7.9 \mathrm{~Hz}, 1 \mathrm{H}), 7.25-6.98(\mathrm{~m}, 8 \mathrm{H}), 6.30(\mathrm{~d}, J=7.1 \mathrm{~Hz}, 0.5 \mathrm{H}), 5.73$ $(\mathrm{s}, 0.5 \mathrm{H}), 5.23(\mathrm{~s}, 1 \mathrm{H}), 4.48-4.19(\mathrm{~m}, 2 \mathrm{H}), 3.66(\mathrm{~d}, J=5.5 \mathrm{~Hz}, 3 \mathrm{H}), 3.62-3.49(\mathrm{~m}, 3 \mathrm{H}), 3.40-$ $3.30(\mathrm{~m}, 1 \mathrm{H}), 3.20-2.86(\mathrm{~m}, 2.5 \mathrm{H}), 2.76(\mathrm{dd}, J=14.5,9.1 \mathrm{~Hz}, 0.5 \mathrm{H}), 1.43(\mathrm{~d}, J=11.6 \mathrm{~Hz}, 9 \mathrm{H})$, $1.27-1.14(\mathrm{~m}, 3 \mathrm{H})$.

${ }^{13} \mathrm{C} \mathrm{NMR}\left(126 \mathrm{MHz}, \mathrm{CDCl}_{3}\right): \delta 173.9 \& 173.7,172.7 \& 172.3,171.2 \& 170.9,155.1,138.03 \&$ $137.96,135.99 \& 135.97,132.2,132.0,129.4,129.1,128.7,128.6,126.98 \& 126.91,122.32 \&$ $122.25,119.88 \& 119.86,118.85 \& 118.82,111.01 \& 110.97,108.6,80.1,52.6 \& 52.5,52.4 \&$ $52.3,48.2,45.0,39.7,28.45,28.41,18.7$.

$\underline{\text { HRMS (ESI) }} \mathrm{m} / z$ : [M-H] $]^{-}$calcd for $\mathrm{C}_{30} \mathrm{H}_{36} \mathrm{~N}_{3} \mathrm{O}_{7} 550.2559$; found 550.2559 .

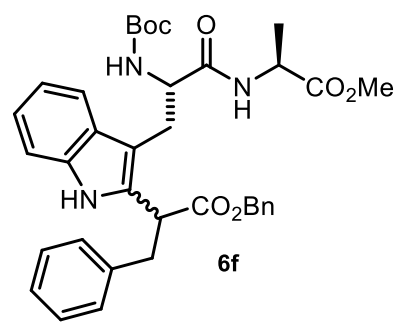

Benzyl 2-(3-((S)-2-((tert-butoxycarbonyl)amino)-3-(((S)-1methoxy-1-oxopropan-2-yl)amino)-3-oxopropyl)-1H-indol-2-yl)3-phenylpropanoate (6f). Prepared according to the general procedure using dipeptide $1 \mathbf{a}(38.9 \mathrm{mg}, 0.1 \mathrm{mmol})$, pyridinium salt $4 \mathbf{e}$ (190 mg, $0.3 \mathrm{mmol})$ and sodium acetate $(16 \mathrm{mg}, 0.2 \mathrm{mmol})$. The product was obtained after purification by column chromatography (gradient hexane/EtOAc 8.2 to $7: 3$ ) as a yellow solid (28.2 $\mathrm{mg}, 45 \%$ yield, mixture of 2 inseparable diastereoisomers in 1:1 ratio).

$\underline{{ }^{1} \mathrm{H} \mathrm{NMR}}\left(300 \mathrm{MHz}, \mathrm{CDCl}_{3}\right) \delta 8.69(\mathrm{~s}, 0.5 \mathrm{H}), 8.56(\mathrm{~s}, 0.5 \mathrm{H}), 7.47(\mathrm{t}, J=8.5 \mathrm{~Hz}, 1 \mathrm{H}), 7.35-7.24$ $(\mathrm{m}, 3 \mathrm{H}), 7.23-6.98(\mathrm{~m}, 10 \mathrm{H}), 6.16(\mathrm{~d}, J=6.9 \mathrm{~Hz}, 0.5 \mathrm{H}), 5.71(\mathrm{~s}, 0.5 \mathrm{H}), 5.18(\mathrm{dd}, J=12.3,6.7$ $\mathrm{Hz}, 2 \mathrm{H}), 4.99$ (dd, $J=12.4,2.2 \mathrm{~Hz}, 1 \mathrm{H}), 4.40(\mathrm{br} \mathrm{s}, 1.5 \mathrm{H}), 4.26(\mathrm{~m}, 1 \mathrm{H}), 3.88-3.74(\mathrm{~m}, 0.5 \mathrm{H})$, $3.51(\mathrm{~d}, J=6.5 \mathrm{~Hz}, 3 \mathrm{H}), 3.35$ (ddd, $J=13.0,8.2,4.8 \mathrm{~Hz}, 1 \mathrm{H}), 3.22-2.94(\mathrm{~m}, 2.5 \mathrm{H}), 2.82$ (dd, $J$ $=14.4,9.0 \mathrm{~Hz}, 0.5 \mathrm{H}), 1.42(\mathrm{~d}, 9 \mathrm{H}), 1.17(\mathrm{dd}, J=7.2,3.4 \mathrm{~Hz}, 3 \mathrm{H})$.

${ }^{13} \mathrm{C} \mathrm{NMR}\left(126 \mathrm{MHz}, \mathrm{CDCl}_{3}\right) \delta 173.1,172.6,172.3,171.1,170.9,137.92,137.86,135.9,135.4$, $135.3,132.2,132.0,130.5,129.5,129.1,129.1,128.9,128.8,128.72,128.67,128.65,128.58$, 
$128.54,128.49,128.41,128.1,128.1,127.0,126.9,126.5,122.6,122.3,122.2,119.9,118.8$, 111.00, 110.96, 109.0, 108.6, 77.4, 77.2, 76.9, 67.6, 67.3, 67.2, 66.5, 60.5, 55.6, 55.3, 53.6, 52.3, $52.3,48.2,48.19,48.15,45.08,40.4,39.9,32.1,30.3,29.83,29.78,29.5,28.43,28.38,27.7,22.8$, $21.2,18.7,18.63,18.57,14.3,14.2$.

HRMS (ESI) $m / z:[\mathrm{M}+\mathrm{Na}]^{+}$calcd for $\mathrm{C}_{36} \mathrm{H}_{41} \mathrm{~N}_{3} \mathrm{NaO}_{7} 650.2837$; found 650.2825 .

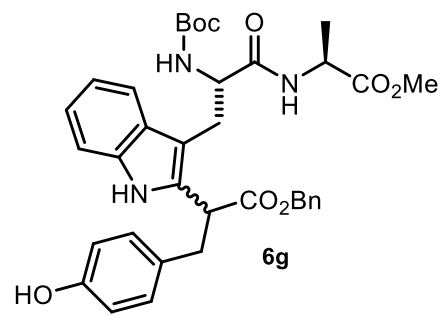

Benzyl 2-(3-((S)-2-((tert-butoxycarbonyl)amino)-3-((S)-1-
methoxy-1-oxopropan-2-yl)amino)-3-oxopropyl)-1H-indol-2-
yl)-3-(4-hydroxyphenyl)propanoate (6g). Prepared according to
the general procedure using dipeptide 1a $(38.9 \mathrm{mg}, 0.1 \mathrm{mmol})$,
pyridinium salt $4 \mathbf{e}(195 \mathrm{mg}, 0.3 \mathrm{mmol})$ and sodium acetate $(16 \mathrm{mg}$,
$0.2 \mathrm{mmol})$. The product was obtained after purification by column
chromatography (gradient hexane/EtOAc 8.2 to $7: 3)$ as a yellow solid (30.0 mg, 47\% yield, mixture of 2 inseparable diastereoisomers in 1:1 ratio).

$\underline{{ }^{1} \mathrm{H} \mathrm{NMR}}\left(300 \mathrm{MHz}, \mathrm{CDCl}_{3}\right) \delta 8.74(\mathrm{~s}, 0.5 \mathrm{H}), 8.66(\mathrm{~s}, 0.5 \mathrm{H}), 7.45(\mathrm{t}, J=7.7 \mathrm{~Hz}, 1 \mathrm{H}), 7.37(\mathrm{~d}, J=$ $1.8 \mathrm{~Hz}, 4 \mathrm{H}), 7.14$ (tdd, $J=6.7,2.9,1.3 \mathrm{~Hz}, 3 \mathrm{H}), 7.08-6.83(\mathrm{~m}, 3.5 \mathrm{H}), 6.72-6.57(\mathrm{~m}, 2.5 \mathrm{H})$, $6.30(\mathrm{~d}, J=7.3 \mathrm{~Hz}, 0.5 \mathrm{H}), 5.90(\mathrm{~s}, 0.5 \mathrm{H}), 5.17(\mathrm{dd}, J=12.2,4.7 \mathrm{~Hz}, 2 \mathrm{H}), 4.99(\mathrm{dd}, J=12.3,3.8$ $\mathrm{Hz}, 1 \mathrm{H}), 4.31(\mathrm{dt}, J=10.3,7.2 \mathrm{~Hz}, 2 \mathrm{H}), 3.88(\mathrm{dd}, J=15.7,8.4 \mathrm{~Hz}, 1 \mathrm{H}), 3.53(\mathrm{~d}, J=20.4 \mathrm{~Hz}$, $3 \mathrm{H}), 3.25$ (ddd, $J=13.4,7.7,5.6 \mathrm{~Hz}, 1 \mathrm{H}), 3.14-3.00(\mathrm{~m}, 2 \mathrm{H}), 2.97-2.79(\mathrm{~m}, 1 \mathrm{H}), 1.40(\mathrm{~s}, 9 \mathrm{H})$, $1.27-1.08(\mathrm{~m}, 3 \mathrm{H})$.

${ }^{13} \mathrm{C} \mathrm{NMR}\left(126 \mathrm{MHz}, \mathrm{CDCl}_{3}\right) \delta 173.3,173.0,172.4,171.4,171.3,155.3,155.2,135.94,135.90$, $135.4,135.3,132.8,132.1,132.0,130.5,130.2,129.7,129.5,128.8,128.74,128.68,128.67$, 128.6, 128.4, 128.21, 128.15, 122.3, 122.2, 119.9, 118.8, 118.7, 116.1, 116.00, 115.94, 115.7, 111.0, 108.8, 77.4, 77.2, 76.9, 67.3, 67.2, 55.6, 53.5, 52.5, 52.4, 52.3, 48.29, 48.25, 48.19, 45.5, $45.4,39.5,39.1,32.1,29.82,29.78,29.5,28.40,28.37,27.6,22.8,18.6,18.5,14.2$.

HRMS (ESI) $m / z:[\mathrm{M}+\mathrm{Na}]^{+}$calcd for $\mathrm{C}_{36} \mathrm{H}_{41} \mathrm{~N}_{3} \mathrm{NaO}_{8}$ 666.2786; found 666.2781.

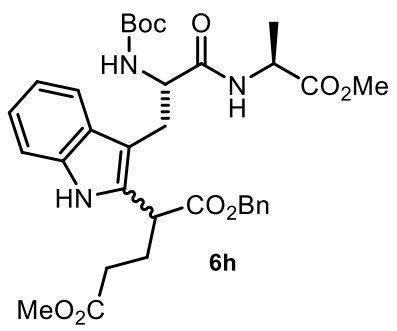

1-Benzyl 5-methyl (S)-2-(3-((S)-2-((tert-butoxycarbonyl)amino)3-(((S)-1-methoxy-1-oxopropan-2-yl)amino)-3-oxopropyl)-1Hindol-2-yl)pentanedioate (6h). Prepared according to the general procedure using dipeptide $\mathbf{1 a}(38.9 \mathrm{mg}, 0.1 \mathrm{mmol})$, pyridinium salt $\mathbf{4 f}$ (189 $\mathrm{mg}, 0.3 \mathrm{mmol})$ and sodium acetate $(16 \mathrm{mg}, 0.2 \mathrm{mmol})$. The product was obtained after purification by column chromatography (gradient hexane/EtOAc 8.2 to $8: 7)$ as a yellow solid (28 $\mathrm{mg}, 45 \%$ yield, mixture of 2 inseparable diastereoisomers in 1:1 ratio).

${ }^{1} \mathrm{H}$ NMR $\left(500 \mathrm{MHz}, \mathrm{CDCl}_{3}\right) \delta 8.53(\mathrm{~s}, 0.5 \mathrm{H}), 8.41(\mathrm{~s}, 0.5 \mathrm{H}), 7.62(\mathrm{~d}, J=7.9 \mathrm{~Hz}, 0.5 \mathrm{H}), 7.52(\mathrm{~d}$, $J=7.9 \mathrm{~Hz}, 0.5 \mathrm{H}), 7.35-7.28(\mathrm{~m}, 7 \mathrm{H}), 7.19-7.07(\mathrm{~m}, 3 \mathrm{H}), 6.45(\mathrm{~s}, 0.5 \mathrm{H}), 6.41(\mathrm{~s}, 0.5 \mathrm{H}), 5.45$ $(\mathrm{d}, J=8.3 \mathrm{~Hz}, 0.5 \mathrm{H}), 5.31(\mathrm{~s}, 0.5 \mathrm{H}), 5.27(\mathrm{dd}, J=28.3,12.3 \mathrm{~Hz}, 1 \mathrm{H}), 5.12(\mathrm{dd}, J=12.3,2.5 \mathrm{~Hz}$, $1 \mathrm{H}), 4.45(\mathrm{q}, J=7.4 \mathrm{~Hz}, 1 \mathrm{H}), 4.41(\mathrm{~s}, 0.5 \mathrm{H}) 4.26(\mathrm{t}, J=7.0 \mathrm{~Hz}, 1 \mathrm{H}), 4.20(\mathrm{~s}, 0.5 \mathrm{H}), 3.65(\mathrm{~d}, J=$ $4.1 \mathrm{~Hz}, 3 \mathrm{H}), 3.60-3.56(\mathrm{~m}, 3 \mathrm{H}), 3.25(\mathrm{~s}, 0.5 \mathrm{H}), 3.16(\mathrm{~s}, 1.5 \mathrm{H}), 2.44-2.27(\mathrm{~m}, 4 \mathrm{H}), 1.39(\mathrm{~s}, 3 \mathrm{H})$, $1.31(\mathrm{~s}, 6 \mathrm{H}), 1.28-1.18(\mathrm{~m}, 3 \mathrm{H})$.

${ }^{13} \mathrm{C} \mathrm{NMR}\left(126 \mathrm{MHz}, \mathrm{CDCl}_{3}\right) \delta 173.5,173.1,172.7,171.3,136.0,135.5,135.4,131.5,128.8$, $128.61,128.57,128.4,128.2,128.1,122.5,122.4,120.0,119.1,118.9,111.0,110.9,109.4,109.1$, 67.6, 67.4, 55.2, 52.4, 52.3, 52.0, 51.9, 48.2, 48.1, 41.9, 41.7, 31.3, 29.8, 28.7, 28.3, 28.0, 27.7, 18.50, 18.45. HRMS (ESI) $\mathrm{m} / z$ : [M-H] $]^{-}$calcd for $\mathrm{C}_{33} \mathrm{H}_{41} \mathrm{~N}_{3} \mathrm{O}_{9}$ 622.2770; found 622.2770 . 


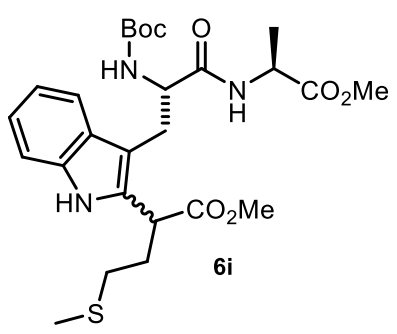

Methyl 2-(3-((S)-2-((tert-butoxycarbonyl)amino)-3-(((S)-1methoxy-1-oxopropan-2-yl)amino)-3-oxopropyl)-1H-indol-2-yl)4-(methylthio)butanoate (6i). Prepared according to the general procedure using dipeptide 1a $(38.9 \mathrm{mg}, 0.1 \mathrm{mmol})$, pyridinium salt KS-Met-OMe (162 mg, $0.3 \mathrm{mmol})$, NaOAc (16.4 mg, $0.2 \mathrm{mmol})$. The crude mixture was purified by column chroatography (hexane/EtOAc 3:2) to afford the product as a yellow solid (15.9 mg, $30 \%$ yield, mixture of 2 inseparable diastereoisomer in 1:1 ratio).

${ }^{1} \mathrm{H} \mathrm{NMR}\left(400 \mathrm{MHz}, \mathrm{CDCl}_{3}\right): 8.51(\mathrm{~s}, 0.5 \mathrm{H}), 8.34(\mathrm{~s}, 0.5 \mathrm{H}), 7.60(\mathrm{~d}, J=7.9 \mathrm{~Hz}, 0.5 \mathrm{H}), 7.52(\mathrm{~d}, J$ $=7.9 \mathrm{~Hz}, 0.5 \mathrm{H}), 7.34-7.27(\mathrm{~m}, 1 \mathrm{H}), 7.20-7.11(\mathrm{~m}, 1 \mathrm{H}), 7.10-7.00(\mathrm{~m}, 1 \mathrm{H}), 6.38(\mathrm{~d}, J=7.0$ $\mathrm{Hz}, 0.5 \mathrm{H}), 6.25(\mathrm{~d}, J=7.2 \mathrm{~Hz}, 0.5 \mathrm{H}), 5.31-5.27(\mathrm{~m}, 1 \mathrm{H}), 4.61-4.08(\mathrm{~m}, 3 \mathrm{H}), 3.75(\mathrm{~d}, J=8.2$ $\mathrm{Hz}, 3 \mathrm{H}), 3.58(\mathrm{~d}, J=10.5 \mathrm{~Hz}, 3 \mathrm{H}), 3.33(\mathrm{dd}, J=14.5,5.6 \mathrm{~Hz}, 0.5 \mathrm{H}), 3.26-3.03(\mathrm{~m}, 1.5 \mathrm{H}), 2.56$ $-2.27(\mathrm{~m}, 3 \mathrm{H}), 2.21-2.09(\mathrm{~m}, 1 \mathrm{H}), 2.07(\mathrm{~s}, 3 \mathrm{H}), 1.39(\mathrm{~d}, J=11.5 \mathrm{~Hz}, 9 \mathrm{H}), 1.30-1.20(\mathrm{~m}, 3 \mathrm{H})$.

${ }^{13} \mathrm{C} \mathrm{NMR}\left(126 \mathrm{MHz}, \mathrm{CDCl}_{3}\right): 173.6,172.7,171.3,155.4,136.05 \& 135.99,132.0,131.8,128.1$, $122.5,120.0,119.2 \& 118.9,110.97 \& 110.91,108.8,80.1,52.8 \& 52.7,52.41 \& 52.37,48.3 \&$ $48.2,41.5,31.7 \& 31.6,29.8,28.4,27.7,18.8 \& 18.6,15.51 \& 15.46$.

HRMS (ESI) $m / z:[\mathrm{M}-\mathrm{H}]^{-}$calcd for $\mathrm{C}_{26} \mathrm{H}_{36} \mathrm{~N}_{3} \mathrm{O}_{7} \mathrm{~S}$ 534.2279; found 534.2290.

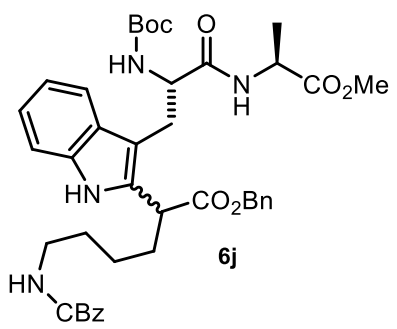

Benzyl 6-(((benzyloxy)carbonyl)amino)-2-(3-((S)-2-((tertbutoxycarbonyl)amino)-3-(((S)-1-methoxy-1-oxopropan-2yl)amino)-3-oxopropyl)-1H-indol-2-yl)hexanoate (6j). Prepared according to the general procedure using dipeptide 1a (38.9 $\mathrm{mg}, 0.1$ mmol), pyridinium salt $\mathbf{4 g}(225 \mathrm{mg}, 0.3 \mathrm{mmol})$ and sodium acetate (16 $\mathrm{mg}, 0.2 \mathrm{mmol})$. The product was obtained after purification by column chromatography (gradient hexane/EtOAc 8.2 to $4: 6$ ) as a yellow solid (20.0 mg, 27\%).

${ }^{1} \mathrm{H}$ NMR $\left(500 \mathrm{MHz}, \mathrm{CDCl}_{3}\right) \delta 8.59(\mathrm{~s}, 0.5 \mathrm{H}), 8.46(\mathrm{~s}, 0.5 \mathrm{H}), 7.51(\mathrm{dd}, J=33.4,7.8 \mathrm{~Hz}, 1 \mathrm{H}), 7.32$ $-7.25(\mathrm{~m}, 11 \mathrm{H}), 7.09(\mathrm{dtd}, J=34.1,7.2,4.7 \mathrm{~Hz}, 2 \mathrm{H}), 6.18(\mathrm{~s}, 0.5 \mathrm{H}), 5.41(\mathrm{~s}, 0.5 \mathrm{H}), 5.33(\mathrm{~s}, 1 \mathrm{H})$, $5.29-5.23(\mathrm{~m}, 1 \mathrm{H}), 5.21(\mathrm{t}, J=2.98,0.5 \mathrm{H}), 5.14-5.01(\mathrm{~m}, 4 \mathrm{H}), 4.94(\mathrm{~s}, 0.5 \mathrm{H}), 4.41(\mathrm{~s}, 0.5 \mathrm{H})$, $4.33(\mathrm{td}, J=8.1,6.2 \mathrm{~Hz}, 0.5 \mathrm{H}), 4.13(\mathrm{~s}, 0.5 \mathrm{H}), 4.06(\mathrm{~s}, 0.5 \mathrm{H}), 3.50(\mathrm{~s}, 2 \mathrm{H}), 3.17-3.09(\mathrm{~m}, 4 \mathrm{H})$, $2.62(\mathrm{~s}, 2 \mathrm{H}), 2.06(\mathrm{sf}, 1 \mathrm{H}), 1.91(\mathrm{~s}, 1 \mathrm{H}), 1.51(\mathrm{dt}, J=14.0,7.1 \mathrm{~Hz}, 2 \mathrm{H}), 1.38(\mathrm{~s}, 9 \mathrm{H}), 1.20(\mathrm{~s}, 3 \mathrm{H})$, $1.18-1.09(\mathrm{~m}, 2 \mathrm{H})$.

${ }^{13} \mathrm{C} \mathrm{NMR}\left(126 \mathrm{MHz}, \mathrm{CDCl}_{3}\right) \delta 156.7,136.7,135.9,135.6,135.5,132.6,132.5,128.82,128.76$, $128.75,128.7,128.61,128.57,128.5,128.3,128.24,128.19,122.2,119.9,118.8,118.7,111.0$, $108.4,80.1,77.4,77.2,76.9,70.4,67.5,67.4,67.2,66.8,52.4,52.3,48.3,48.2,46.0,42.8,40.6$, $33.9,32.1,29.8,29.5,28.4,24.4,22.8,22.0,18.5,14.2,8.8$.

HRMS (ESI) $m / z:[\mathrm{M}+\mathrm{Na}]^{+}$calcd for $\mathrm{C}_{41} \mathrm{H}_{50} \mathrm{~N}_{4} \mathrm{NaO}_{9}$ 765.3470; found 765.3459. 


\section{G. 1 mmol scale reaction}

To demonstrate the practical utility of our method, we conducted the model reaction on a $1 \mathrm{mmol}$ scale. The reaction performed with minor loss of efficiency, affording the desired product in $55 \%$.<smiles>COC(=O)C(C)NC(=O)C(Cc1c[nH]c2ccccc12)N[13C](=O)c1ccccc1</smiles>

1a

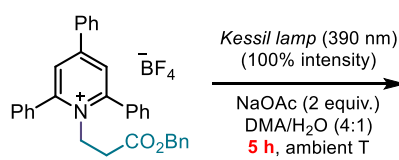

4b<smiles>[Y4]C(NC(=O)C(Cc1c(CC(=O)Br)[nH]c2ccccc12)NC(=O)c1ccccc1)C(=O)OC</smiles>

$\mathbf{5 b}$

Procedure for $1 \mathrm{mmol}$ scale reaction: To a $50 \mathrm{~mL}$ argon purged Schlenck tube, containing the dipeptide $1 \mathbf{a}$ ( $389 \mathrm{mg}, 1$ equiv, $1.00 \mathrm{mmol}$ ), the pyridinium salt $\mathbf{4 b}(1.63 \mathrm{~g}, 3$ equiv, $3.00 \mathrm{mmol})$, and sodium acetate (164 mg, 2 equiv, $2.00 \mathrm{mmol}$ ) was added argon-sparged DMA (4 mL) and argon-sparged distilled Water $(1 \mathrm{~mL})$. The vial was sealed with parafilm and irradiated for 5 hours with Kessil lamp (390 nm, 100\% intensity, $3 \mathrm{~cm}$ away from the vial) at ambient temperature. The resulting crude mixture was diluted with EtOAc $(100 \mathrm{~mL})$, washed with brine $(2 \times 200 \mathrm{~mL})$, dried over $\mathrm{MgSO}_{4}$ and concentrated under rotary evaporation. The crude mixture was purified by column chromatography (EtOAc/hexane gradient from $20 \%$ EtOAc to $40 \%$ ) to afford product $\mathbf{5 b}$ (295.9 $\mathrm{mg}, 550.4 \mu \mathrm{mol}, 55.0 \%$ ) as a yellow solid.

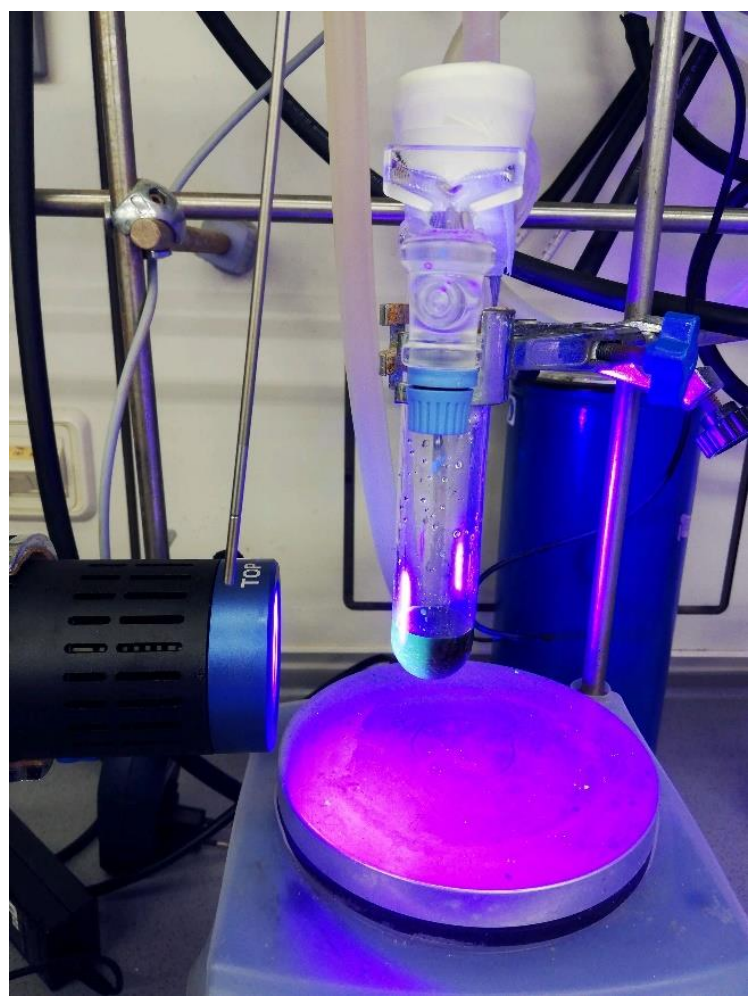

Figure S3. Experimental setup for the $1 \mathrm{mmol}$ scale reaction. 


\section{H. Unsuccessful substrates}
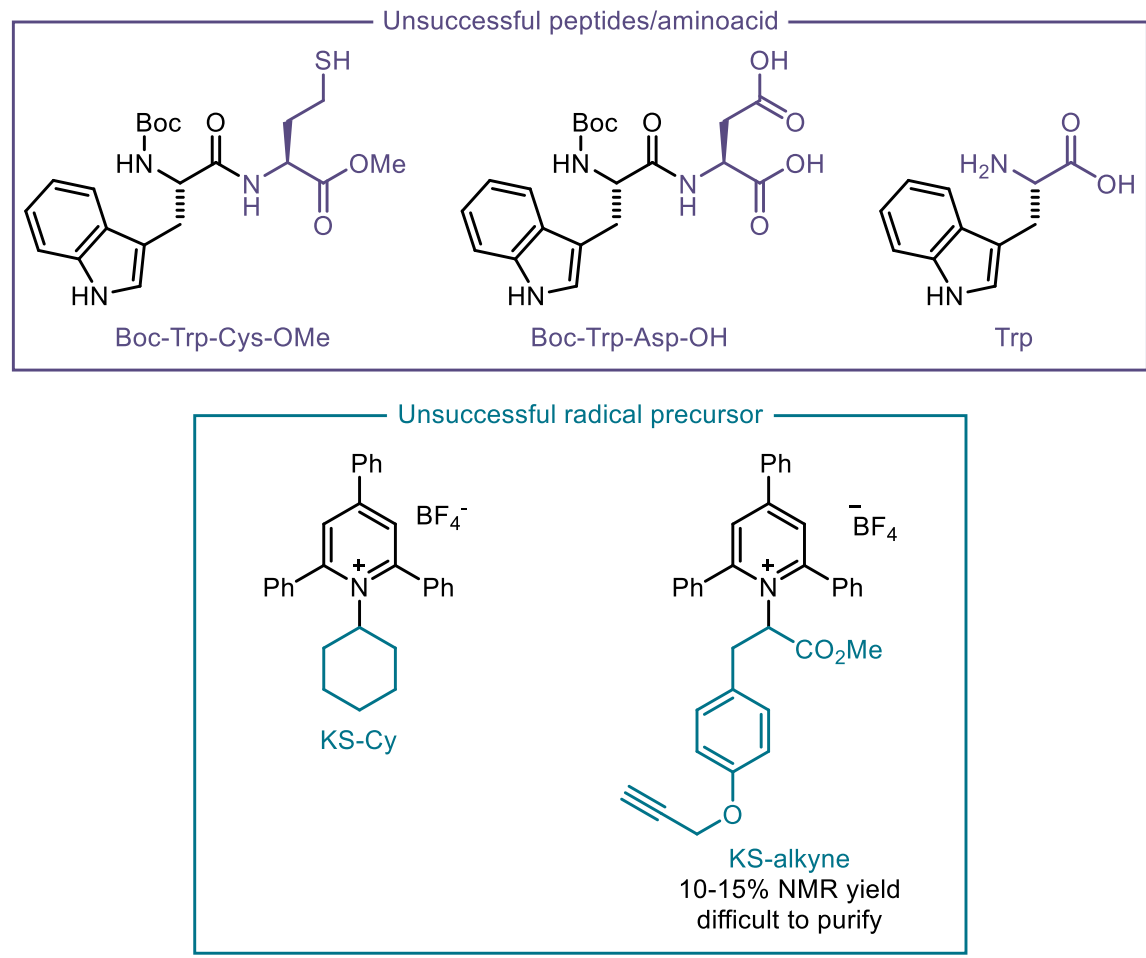

Figure S4. Unsuccessful substrates 


\section{Possible chain mechanism}

While we propose a mechanism based on radical combination within the solvent cage (Figure 1c in the main manuscript), a radical chain propagation proceeding via the addition of a radical into the indole nucleus cannot be excluded. This alternative chain propagation manifold is shown in Scheme S1, where the radical attacks the indole moiety of the tryptophan forming radical S4. The latter propagates the chain reaction through SET to the pyridinium salt. We could not collect evidence to clearly discriminate among these reaction mechanisms since our attempts to determine the quantum yield of the model reaction were frustrated by the heterogeneity of the reaction mixture ( $\mathrm{NaOAc}$ is partially insoluble in the reaction mixture), which precluded a homogeneous illumination, a crucial requirement for a reliable quantum yield determination.

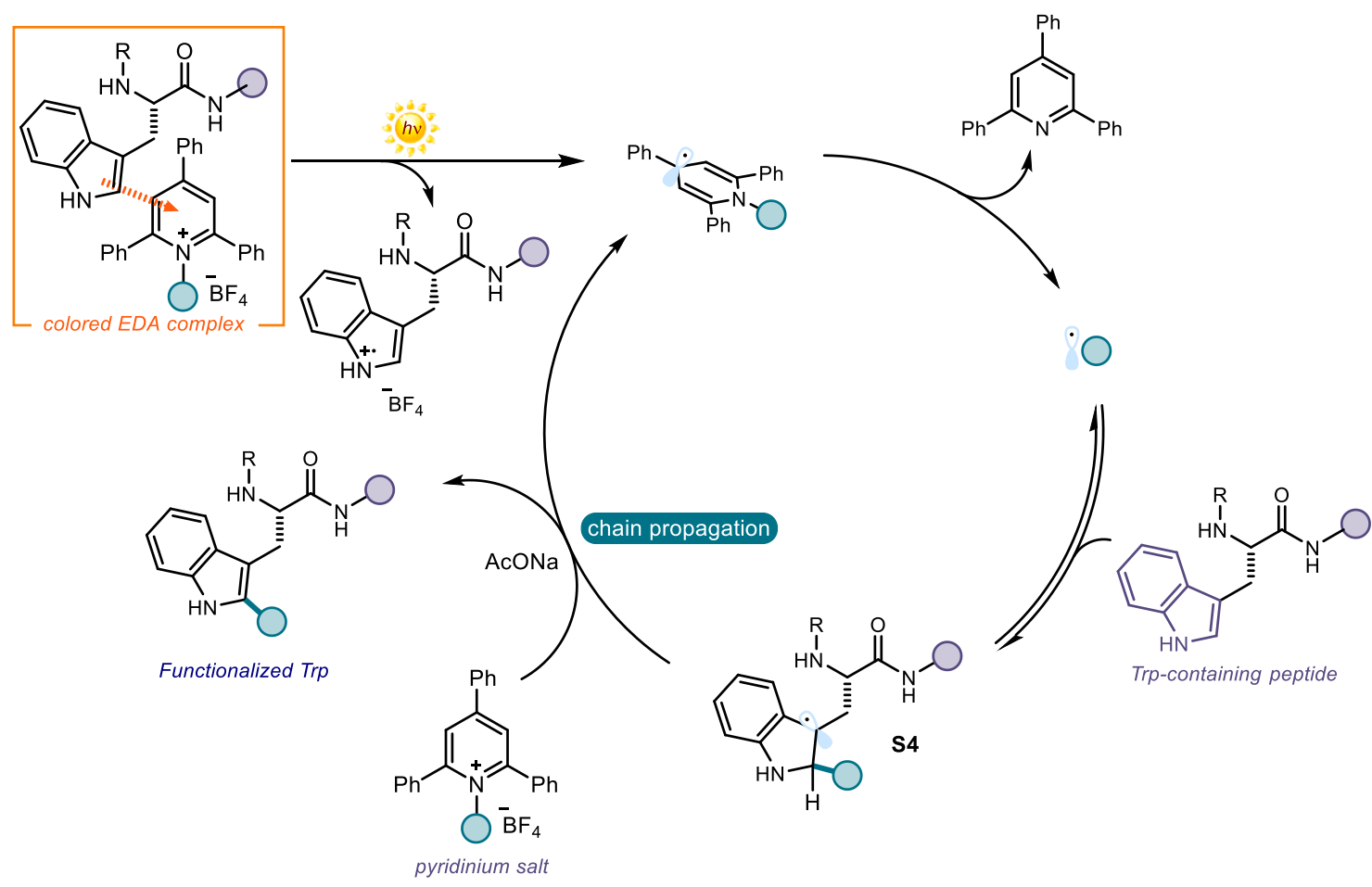

Scheme S1. Possible chain mechanism 


\section{J. References}

1. F. Xue, C. T. Seto, J. Med. Chem. 2005, 48 (22), 6908-6917.

2. H.-C. Chien, C. Colas, K. Finke, S. Springer, L. Stoner, A. A. Zur, B. Venteicher, J. Campbell, C. Hall, A. Flint, E. Augustyn, C. Hernandez, N. Heeren, L. Hansen, A. Anthony, J. Bauer, D. Fotiadis, A. Schlessinger, K. M. Giacomini, A. A. Thomas, J. Med. Chem. 2018, 61 (16), 7358-7373

3. S. Verhoog, C. W. Kee, Y. Wang, T. Khotavivattana, T. C. Wilson, V. Kersemans ,S. Smart, M. Tredwell, B. G. Davis, V. Gouverneur, J. Am. Chem. Soc. 2018, 140 (5), 1572-1575.

4. A. Mahindra, K. Nooney, S. Uraon, K. K. Sharma, R. Jain, RSC Adv. 2013, 3, 16810-16816.

5. J.-S. Suppo, G. Subra, M. Bergès, R. M. de Figueiredo, J.-M. Campagne, Angew. Chem. Int. Ed. 2014, 53, 5389-5393.

6. C. J. Chapman, A. Matsuno, C. G. Frost, and M. C. Willis, Chem. Commun. 2007, 39033905.

7. H.-C. Chien, C. Colas, K. Finke, S. Springer, L. Stoner, A. A. Zur, B. Venteicher, J. Campbell, C. Hall, A. Flint, E. Augustyn, C. Hernandez, N. Heeren, L. Hansen, A. Anthony, J. Bauer, D. Fotiadis, A. Schlessinger, K. M. Giacomini, A. A. Thomas, J. Med. Chem. 2018, 61 (16), 7358-7373

8. C. J. Nalbandian, E. M. Miller, S. T. Toenjes, J. L. Gustafson, Chem. Commun. 2017, 53, 1494.

9. S. Verhoog, C. W. Kee, Y. Wang, T. Khotavivattana, T. C. Wilson, V. Kersemans ,S. Smart, M. Tredwell, B. G. Davis, V. Gouverneur, J. Am. Chem. Soc. 2018, 140 (5), 1572-1575.

10. K. Sugimoto, N. Yamamoto, D. Tominaga, Y. Matsuya, Org. Lett. 2015, 17, 1320-1323. 


\section{K. NMR Spectra}

${ }^{1} \mathrm{H}$ NMR of 3a $\left(500 \mathrm{MHz}, \mathrm{CDCl}_{3}\right)$

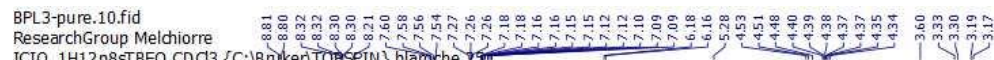
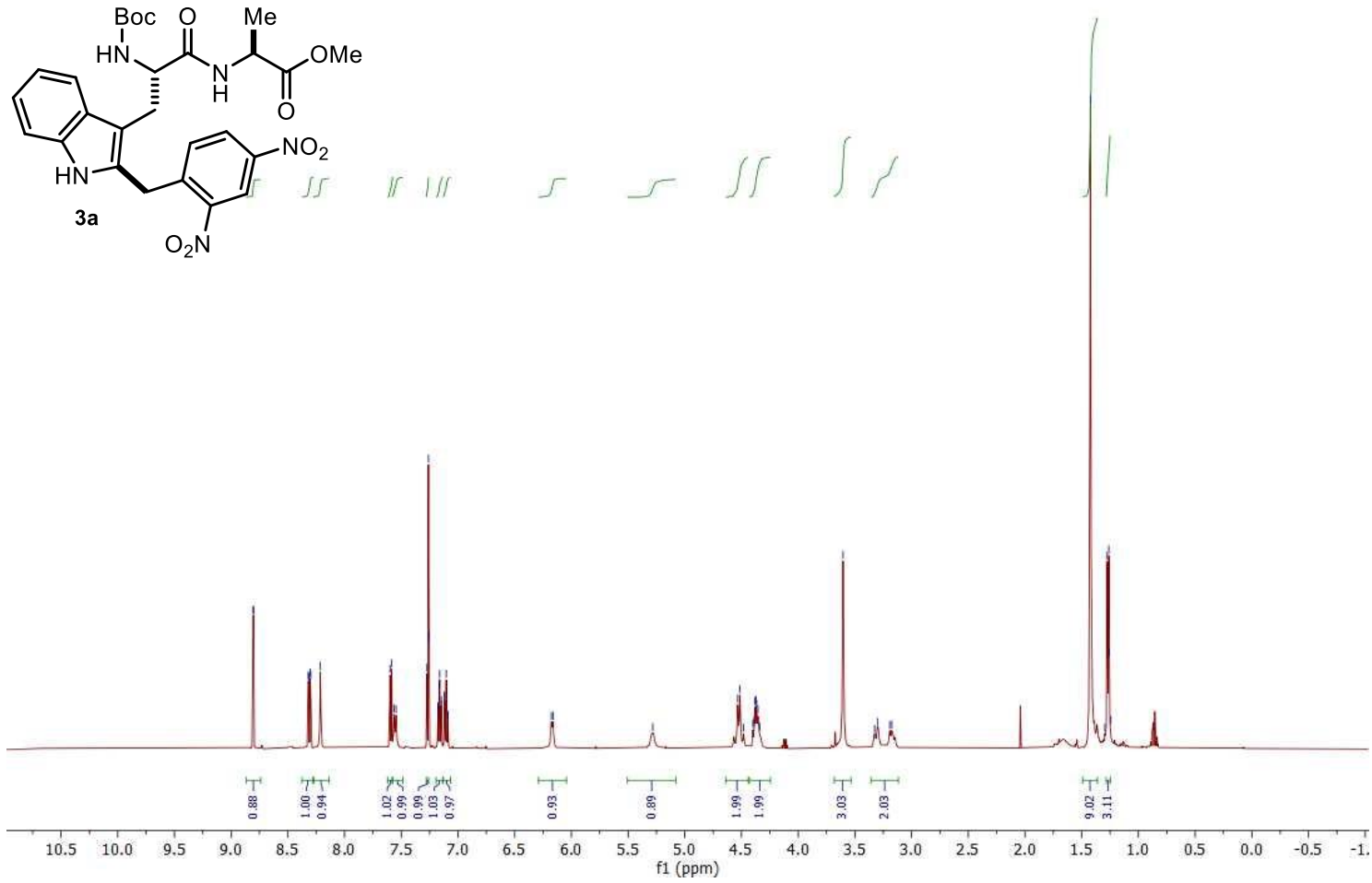

${ }^{13} \mathrm{C}$ NMR of 3a $\left(126 \mathrm{MHz}, \mathrm{CDCl}_{3}\right)$

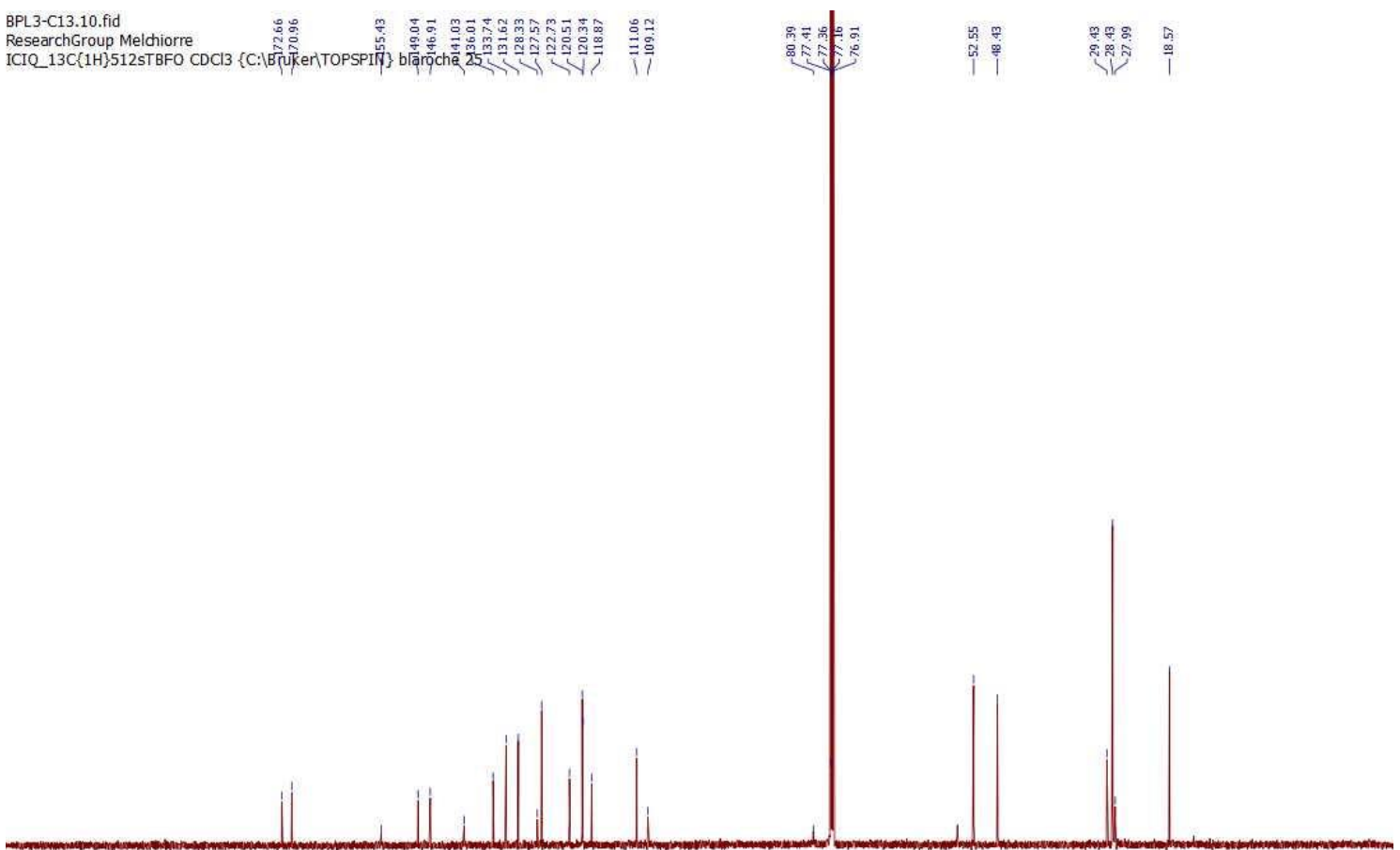

\begin{tabular}{lllllllllllllllllllllllllll}
\hline 20 & 210 & 200 & 190 & 180 & 170 & 160 & 150 & 140 & 130 & 120 & 110 & 100 & 90 & 1 & 10 & 70 & 60 & 50 & 40 & 30 & 20 & 10 & 0 & -10 & -2
\end{tabular} 
${ }^{1} \mathrm{H}$ NMR of $\mathbf{1 b}\left(400 \mathrm{MHz}, \mathrm{C}_{3} \mathrm{D}_{7} \mathrm{NO}\right)$

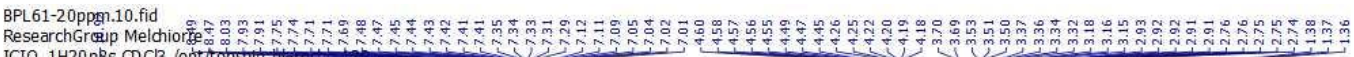

ICIQ $1 \mathrm{H} 20 \mathrm{pBs}$ CDCl3 /opt/topspotit

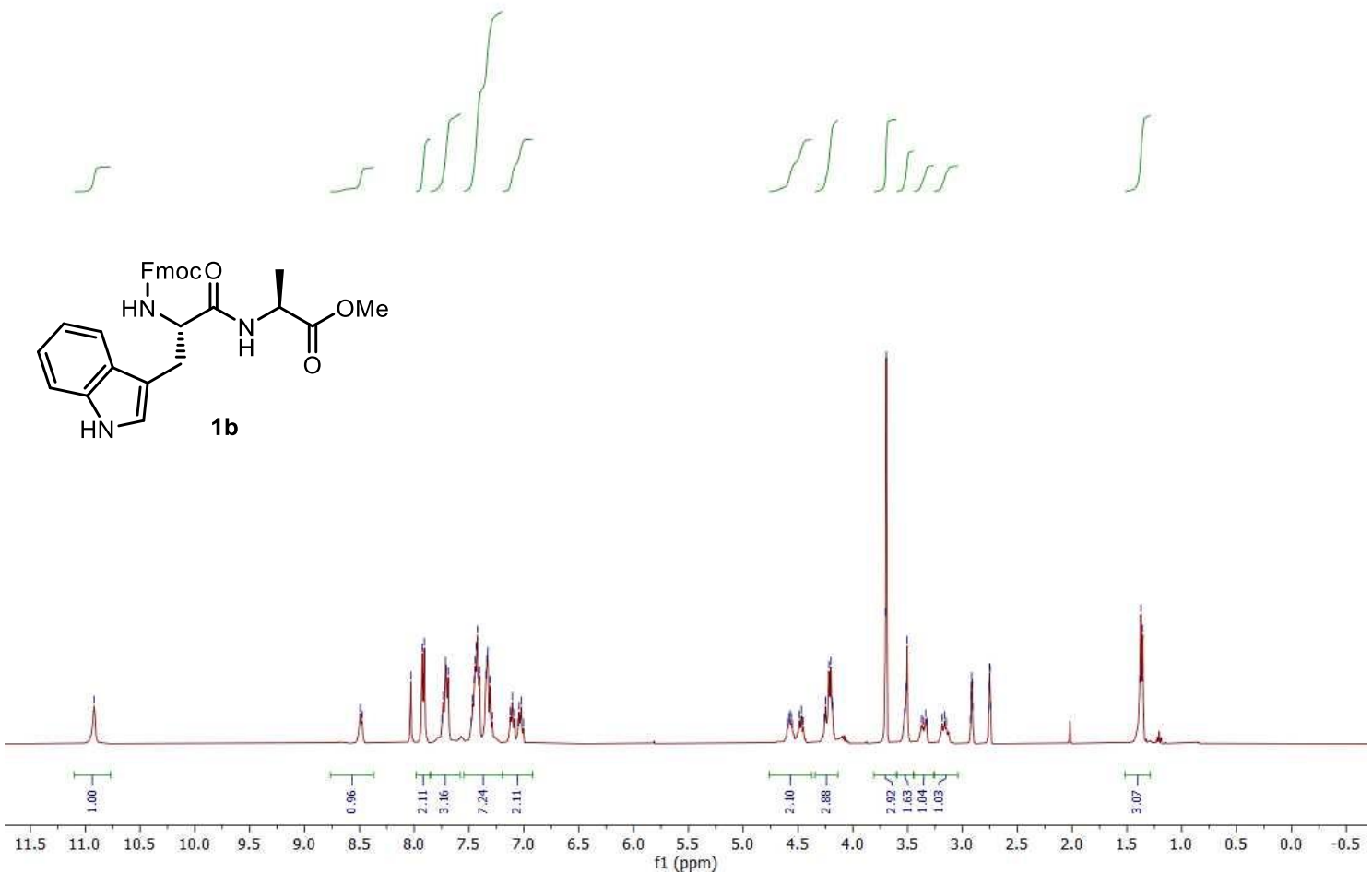

${ }^{13} \mathrm{C}$ NMR of $\mathbf{1 b}\left(101 \mathrm{MHz}, \mathrm{C}_{3} \mathrm{D}_{7} \mathrm{NO}\right)$

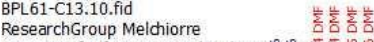

ICIQ_13C\{1H\}512s DMF/opt/topspinin

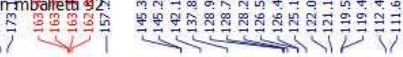
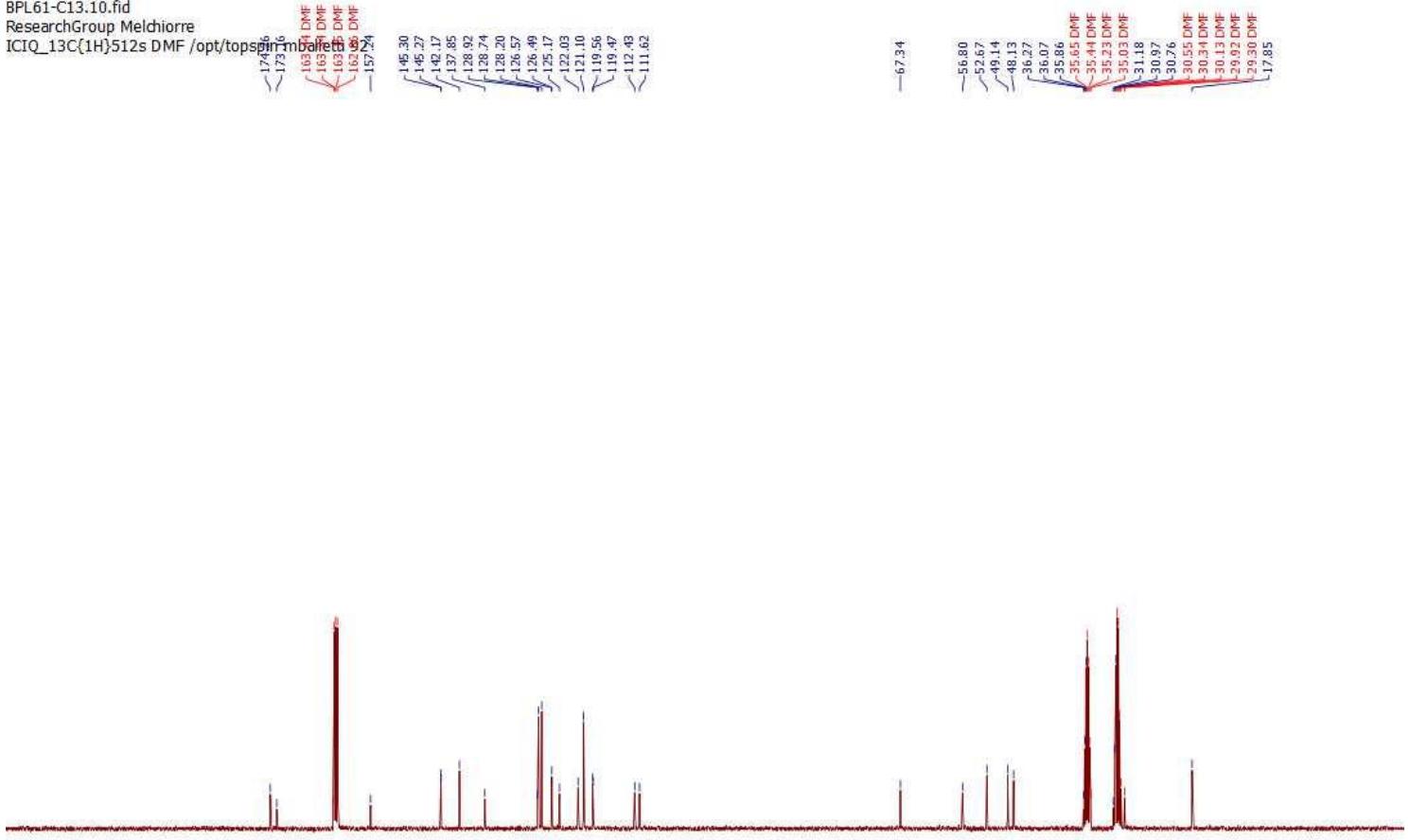

$\begin{array}{llllllllllllllllllllllllllllll}210 & 200 & 190 & 180 & 170 & 160 & 150 & 140 & 130 & 120 & 110 & 100 & 90 & 80 & 70 & 60 & 50 & 40 & 30 & 20 & 10 & 0 & -10\end{array}$ 
${ }^{1} \mathrm{H} \mathrm{NMR}$ of $1 \mathrm{~g}\left(500 \mathrm{MHz}, \mathrm{CDCl}_{3}\right)$

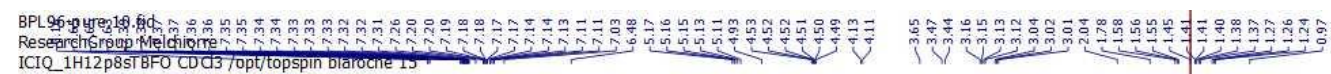<smiles>CCc1c[nH]c2ccccc12</smiles>

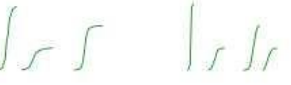

i dili

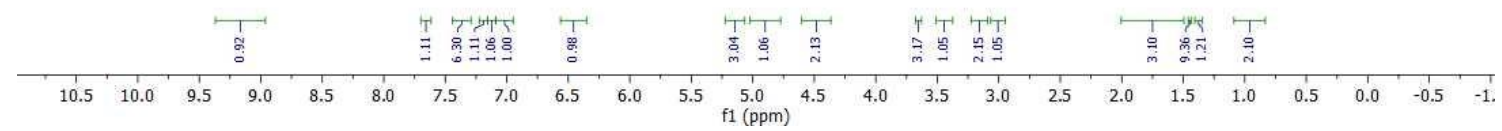

${ }^{13} \mathrm{C}$ NMR of 1 g $\left(126 \mathrm{MHz}, \mathrm{CDCl}_{3}\right)$

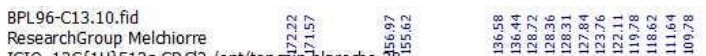

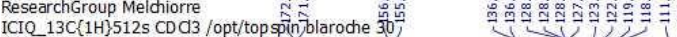

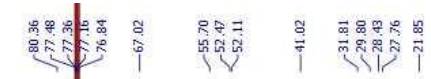

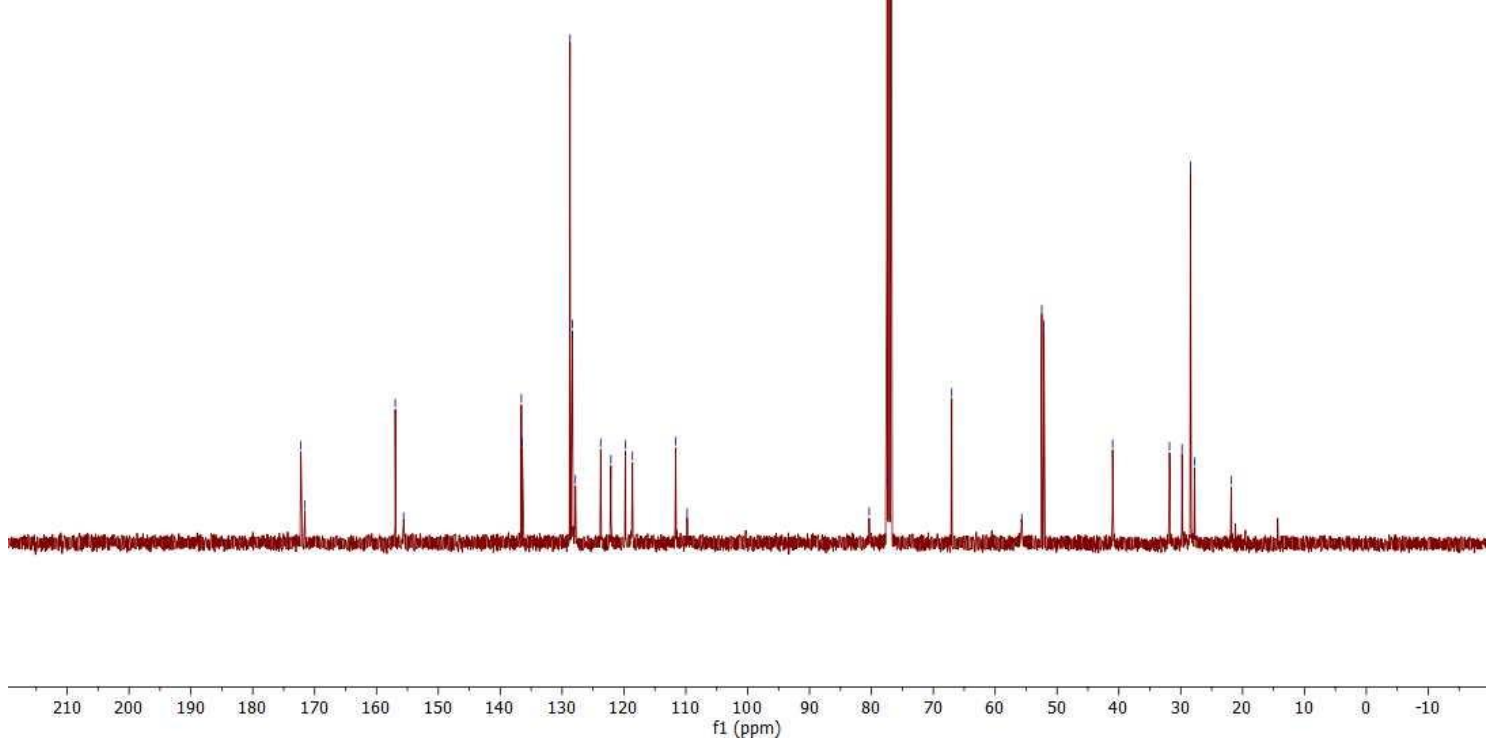


${ }^{1} \mathrm{H} \mathrm{NMR}$ of $\mathbf{S 3}\left(400 \mathrm{MHz}, \mathrm{CDCl}_{3}\right)$

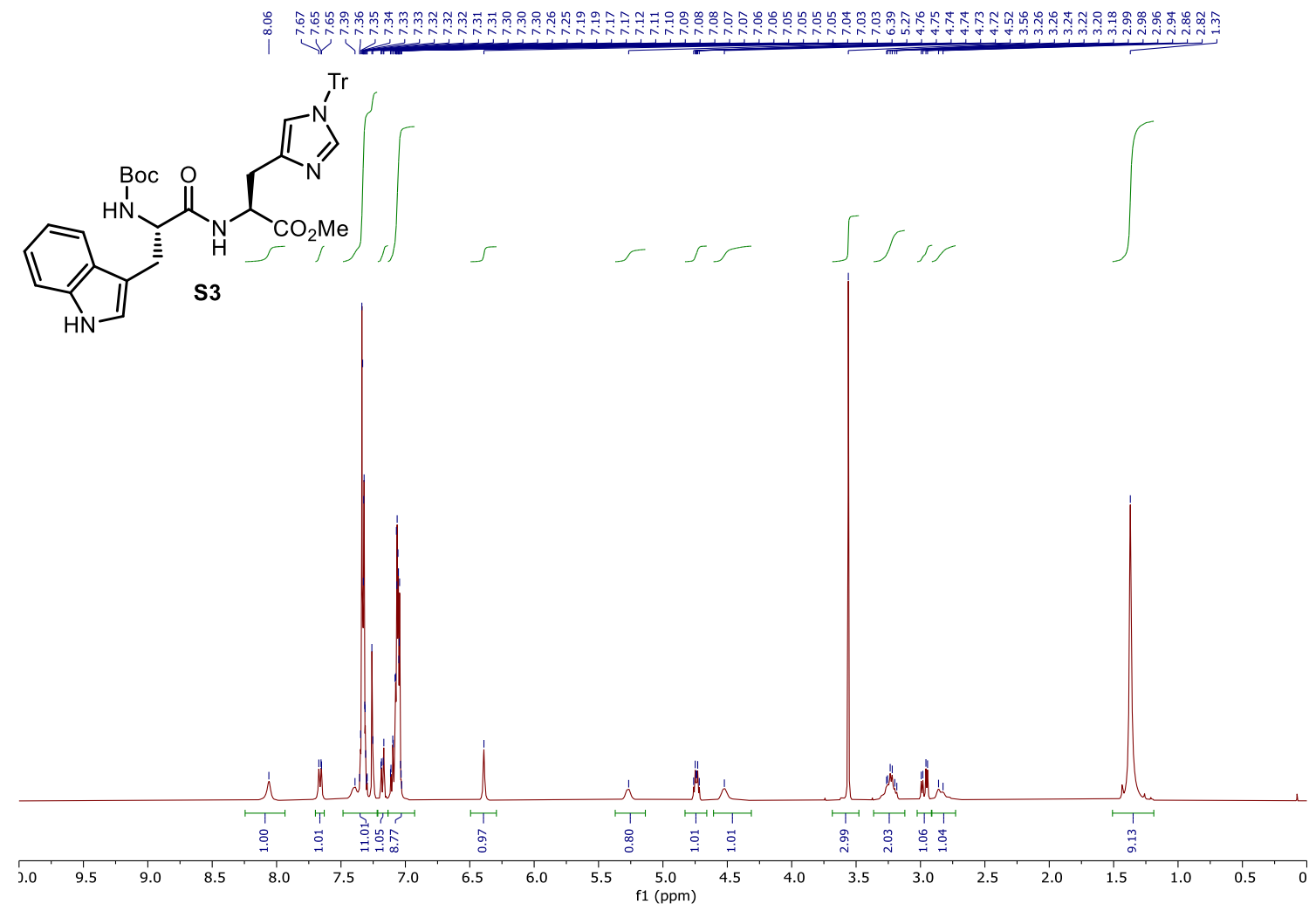

${ }^{13} \mathrm{C}$ NMR of $\mathbf{S 3}\left(101 \mathrm{MHz}, \mathrm{CDCl}_{3}\right)$

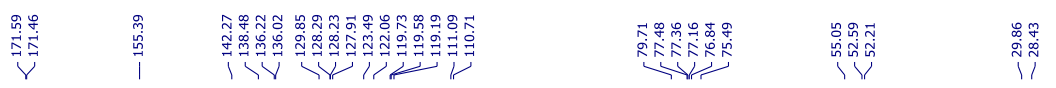

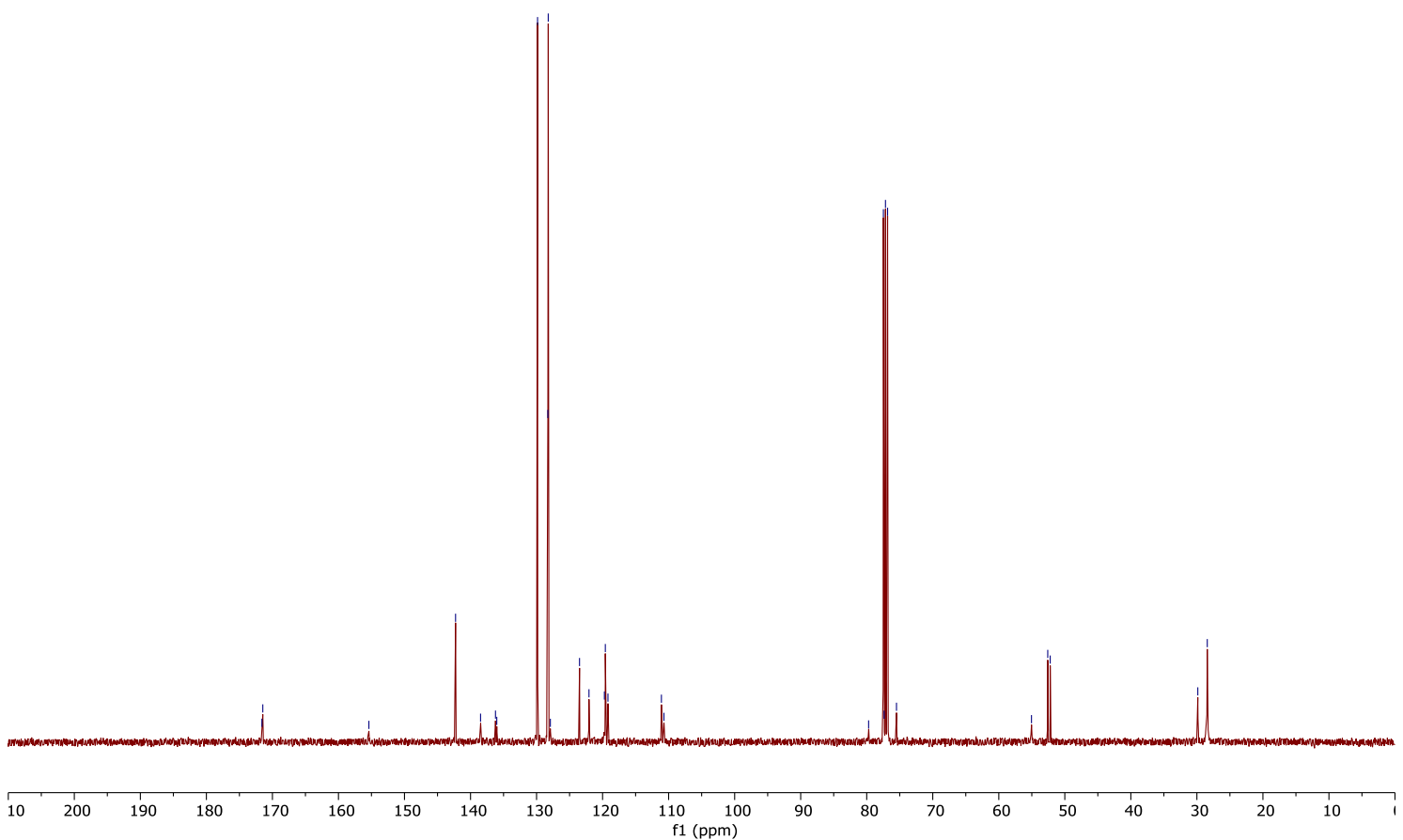


${ }^{1} \mathrm{H} \mathrm{NMR}$ of $\mathbf{1 j}\left(500 \mathrm{MHz}, \mathrm{CDCl}_{3}\right)$

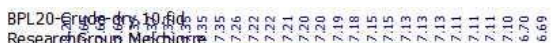

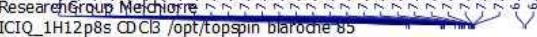<smiles>COC(=O)C(CO)NC(=O)[C@H](Cc1c[nH]c2ccccc12)NC(=O)c1ccccc1</smiles>

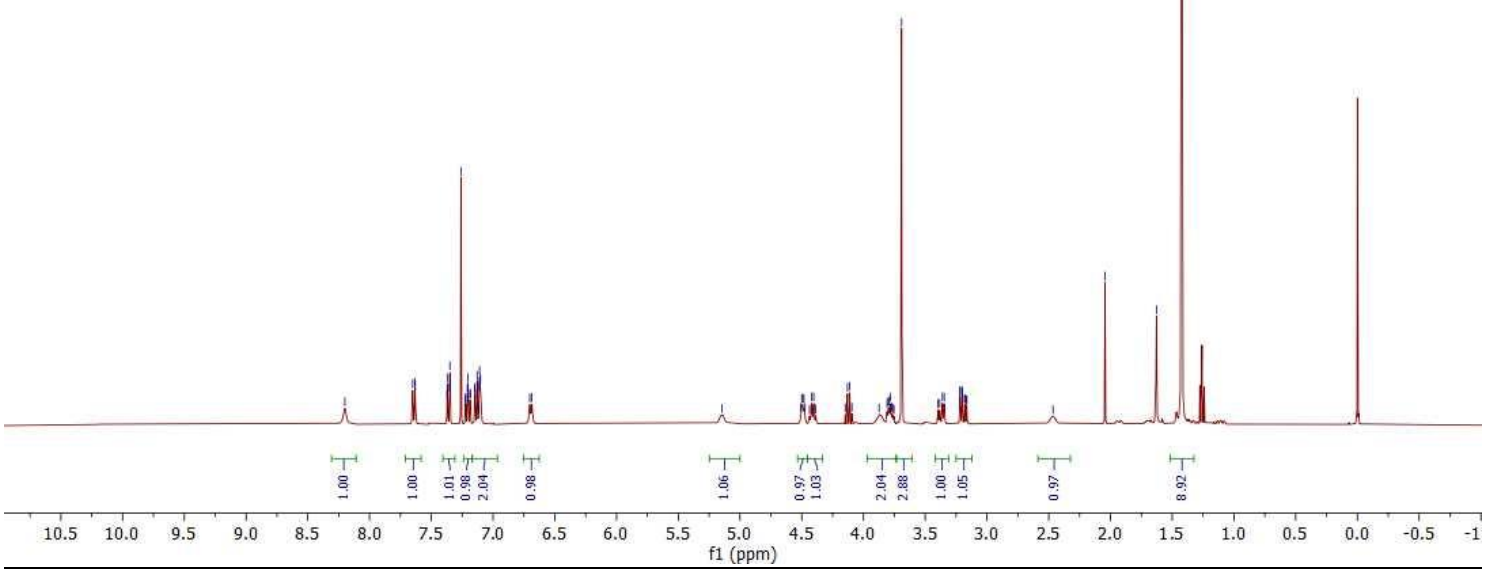

${ }^{13} \mathrm{C}$ NMR of $\mathbf{1 j}\left(126 \mathrm{MHz}, \mathrm{CDCl}_{3}\right)$

BPL20-C13.10.fid

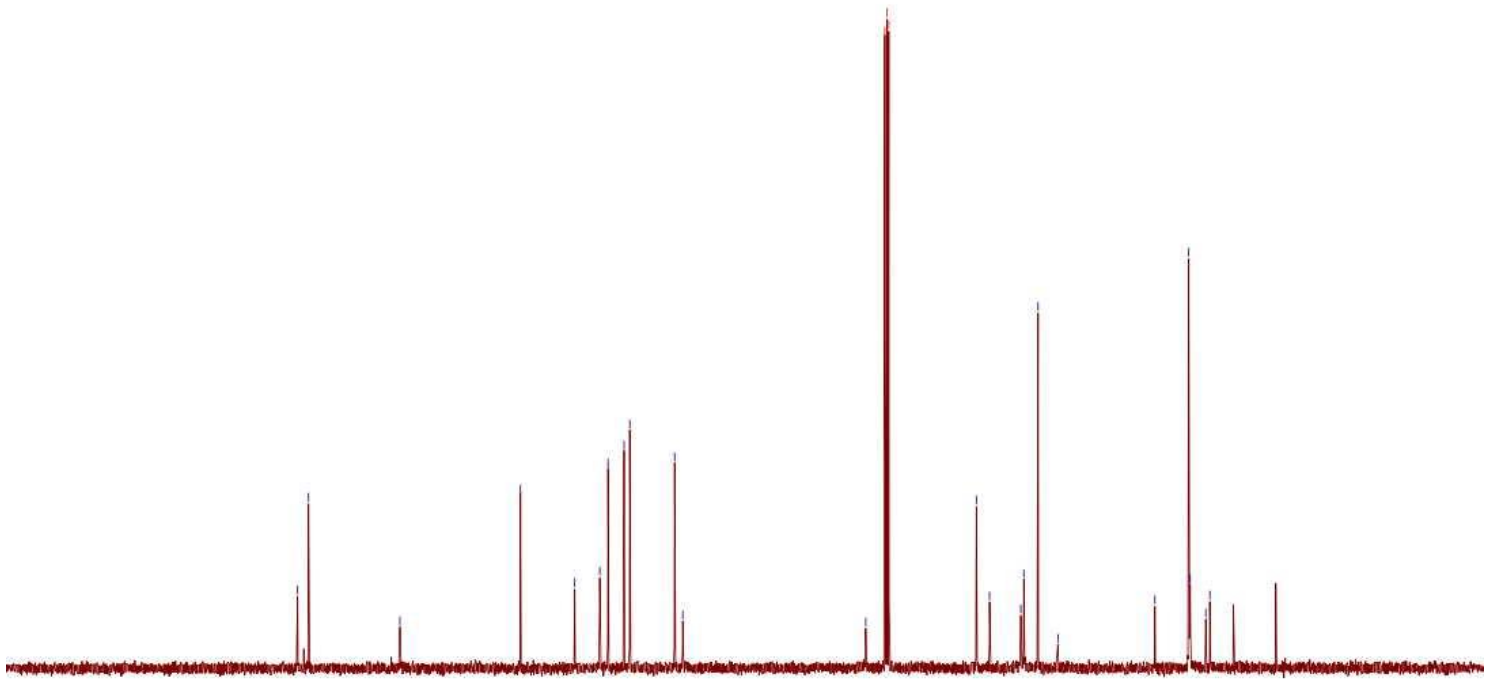

$\begin{array}{llllllllllllllllllllllllll}210 & 200 & 190 & 180 & 170 & 160 & 150 & 140 & 130 & 120 & 110 & 100 & 90 & 80 & 70 & 60 & 50 & 40 & 30 & 20 & 10 & 0 & -10\end{array}$


${ }^{1} \mathrm{H} \mathrm{NMR}$ of $10\left(300 \mathrm{MHz}, \mathrm{CDCl}_{3}\right)$

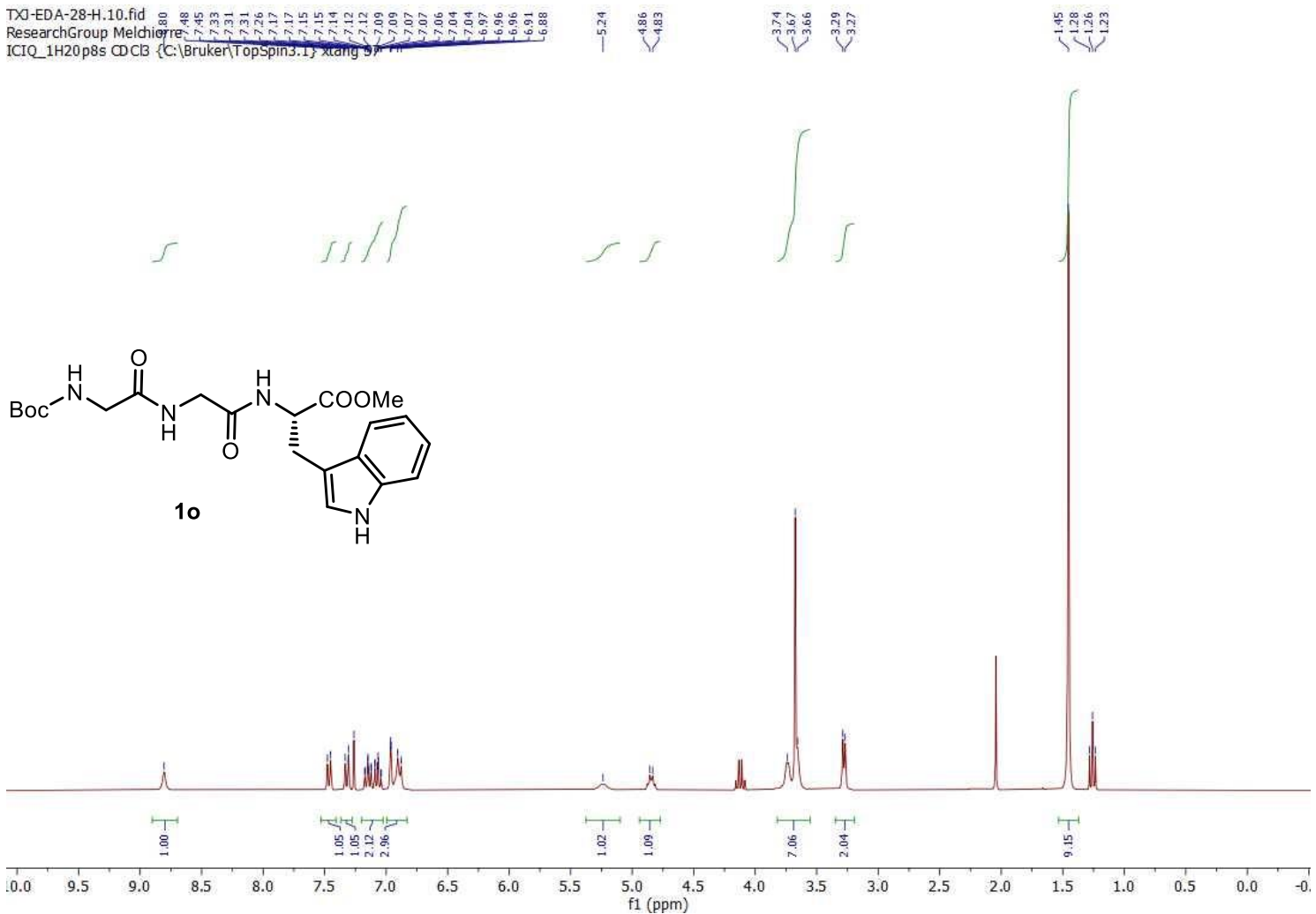

${ }^{13} \mathrm{C}$ NMR of $10\left(126 \mathrm{MHz}, \mathrm{CDCl}_{3}\right)$

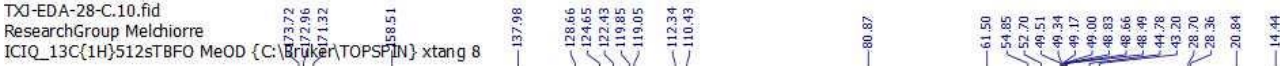

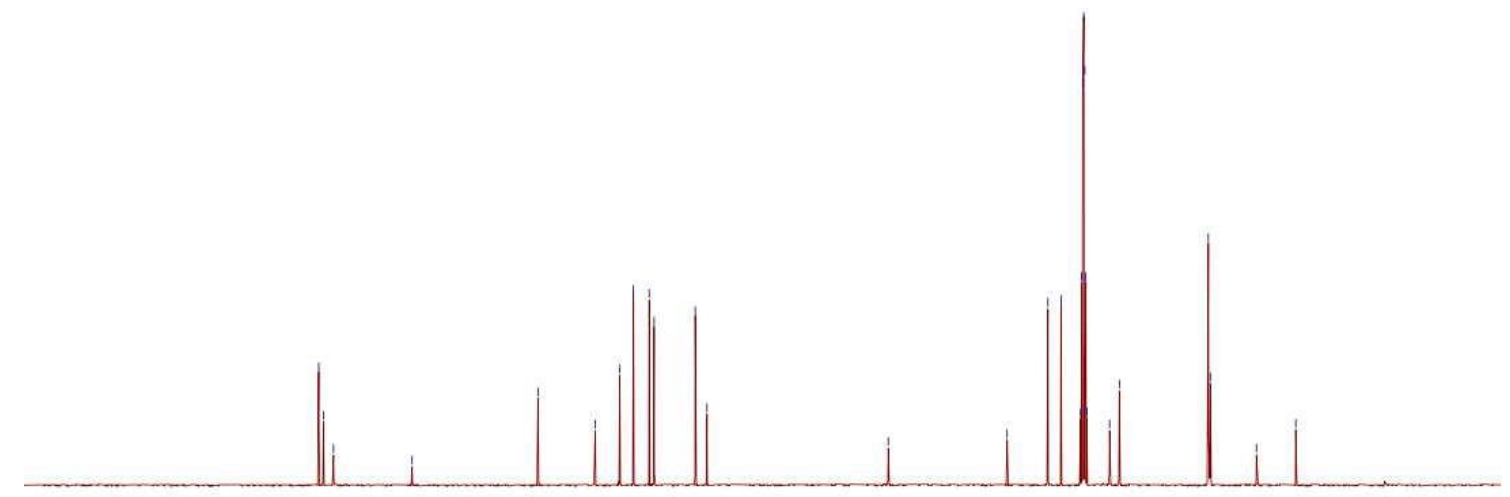

$\begin{array}{llllllllllllllllllllllllllll}720 & 210 & 200 & 190 & 180 & 170 & 160 & 150 & 140 & 130 & 120 & 110 & 100 & 90 & 80 & 70 & 60 & 50 & 40 & 30 & 20 & 10 & 0 & -10\end{array}$ 
${ }^{1} \mathrm{H} \mathrm{NMR}$ of $\mathbf{1 p}\left(300 \mathrm{MHz}, \mathrm{CDCl}_{3}\right)$

TX-EDA-027-HH.10.fid

ICIQ $1 \mathrm{H} 20 \mathrm{p} 8 \mathrm{~S}$ CDCl3 \{C:|Bruker|TopSpin3.1\} xtang 51

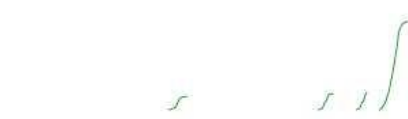<smiles>COC(=O)[C@H](CC(C)C)NC(=O)[C@H](Cc1c[nH]c2ccccc12)NC(=O)CNC(=O)CNC(=O)OCc1ccccc1</smiles>
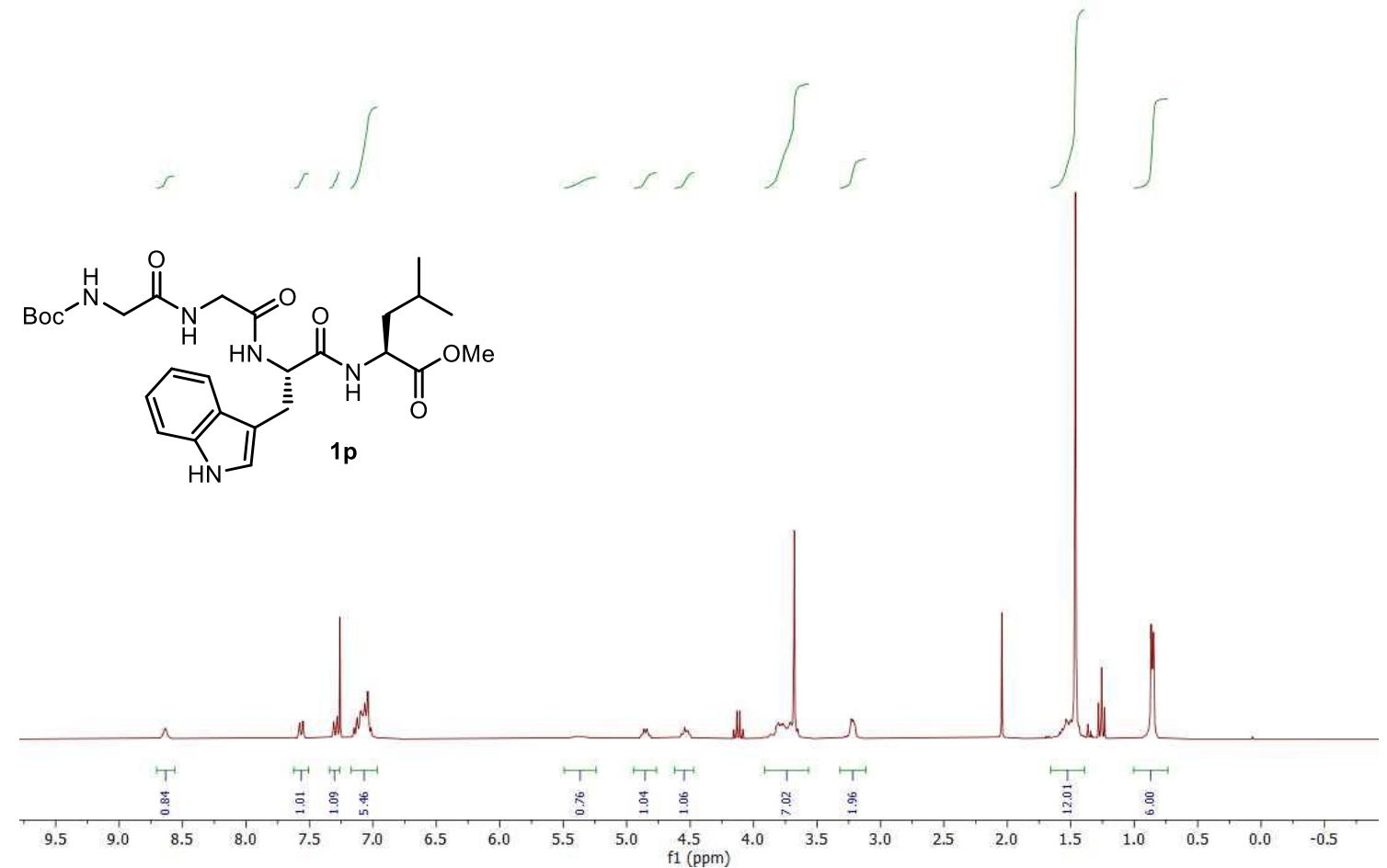

${ }^{13} \mathrm{C}$ NMR of $1 \mathbf{p}\left(126 \mathrm{MHz}, \mathrm{CDCl}_{3}\right)$

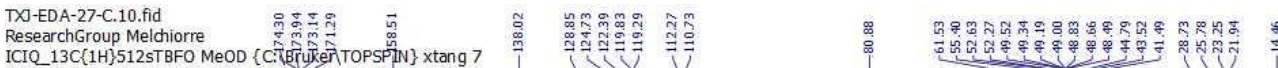

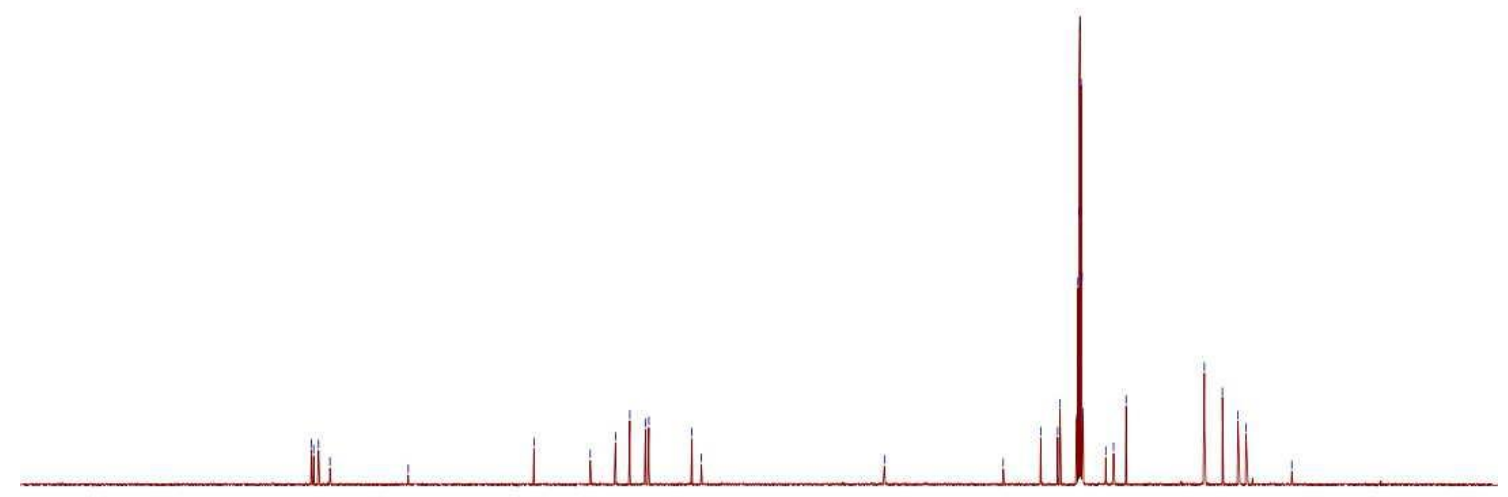

$\begin{array}{lllllllllllllllllllllllllll}120 & 210 & 200 & 190 & 180 & 170 & 160 & 150 & 140 & 130 & 120 & 110 & 100 & 90 & 80 & 70 & 60 & 50 & 40 & 30 & 20 & 10 & 0 & -10\end{array}$ 
${ }^{1} \mathrm{H}$ NMR of $\mathbf{4 b}\left(400 \mathrm{MHz}, \mathrm{CDCl}_{3}\right)$

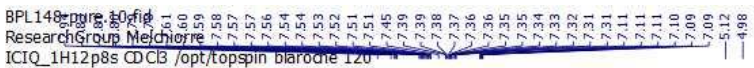
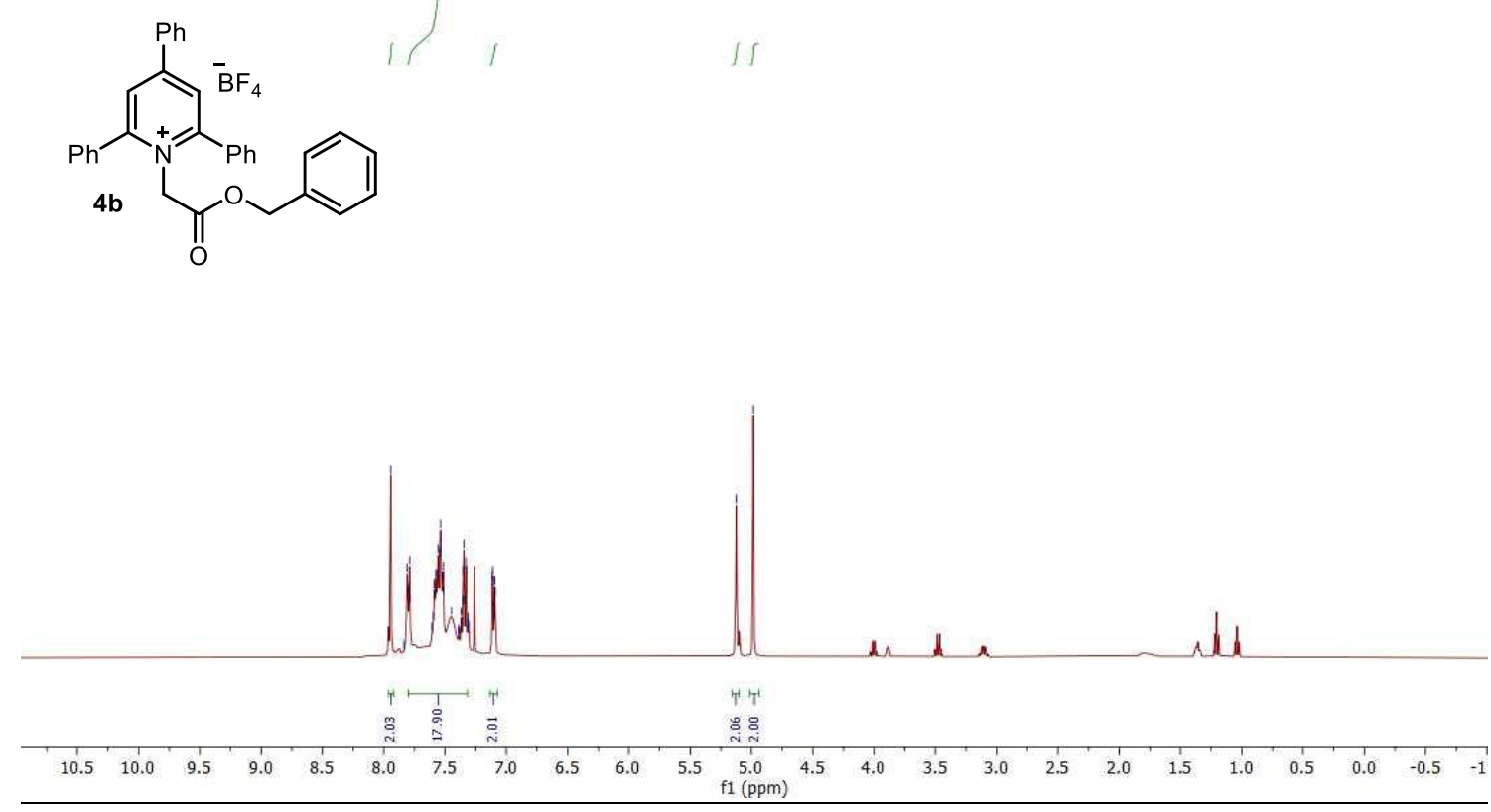

${ }^{13} \mathrm{C}$ NMR of $\mathbf{4 b}\left(101 \mathrm{MHz}, \mathrm{CDCl}_{3}\right)$

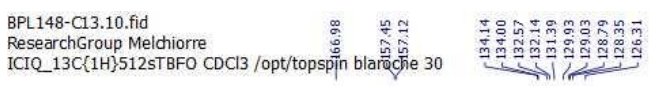

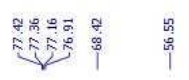

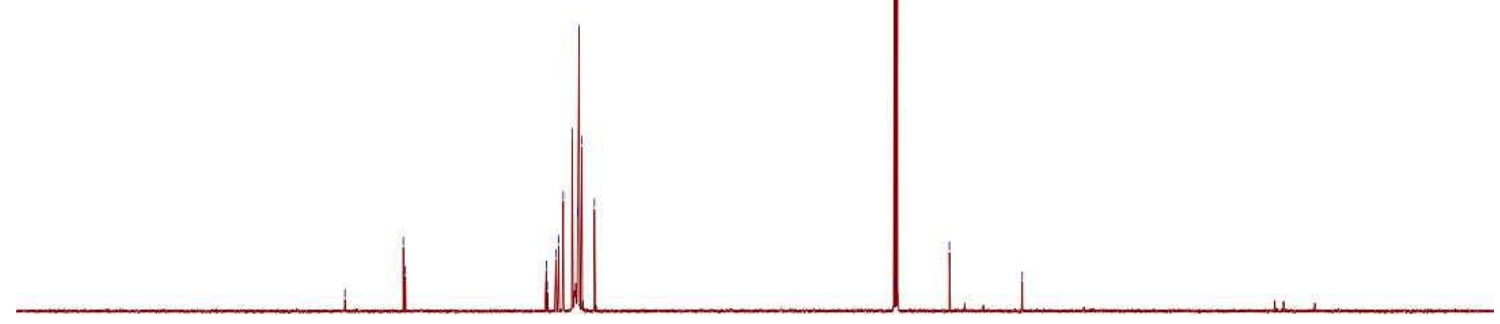

\begin{tabular}{llllllllllllllllllllllllllll}
\hline 20 & 210 & 200 & 190 & 180 & 170 & 160 & 150 & 140 & 130 & 120 & 110 & $\begin{array}{c}100 \\
\mathrm{f} 1(\mathrm{ppm})\end{array}$ & 90 & 80 & 70 & 60 & 50 & 40 & 30 & 20 & 10 & 0 & -10 & -2 \\
\hline
\end{tabular}

S43 
${ }^{1} \mathrm{H}$ NMR of $4 \mathbf{c}\left(400 \mathrm{MHz}, \mathrm{CDCl}_{3}\right)$

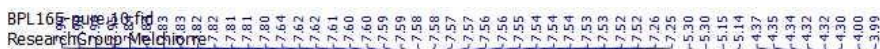

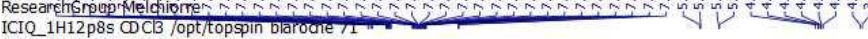<smiles></smiles>

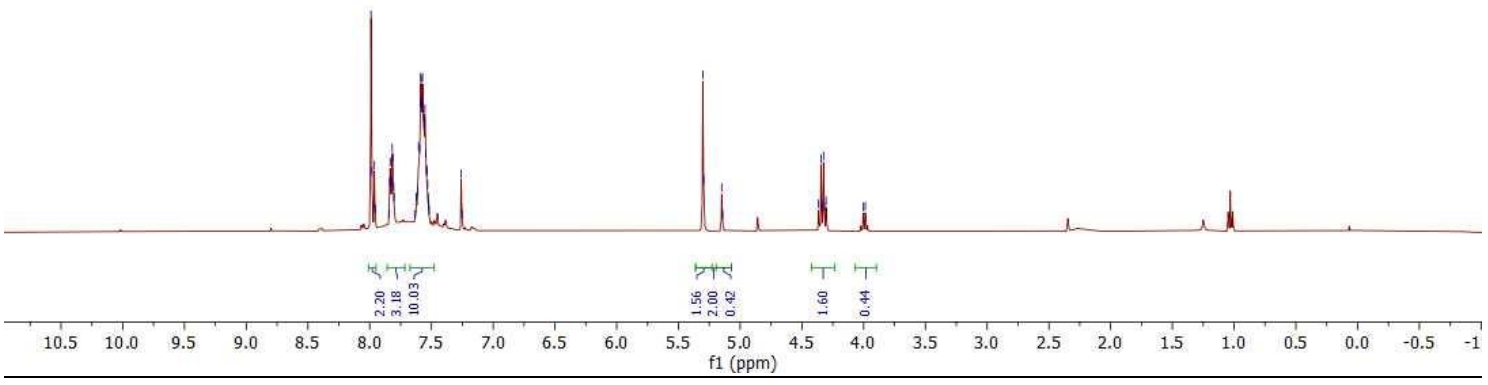

${ }^{13} \mathrm{C}$ NMR of $4 \mathbf{c}\left(101 \mathrm{MHz}, \mathrm{CDCl}_{3}\right)$

BPL165-C13.10. fid

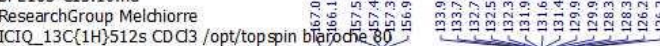

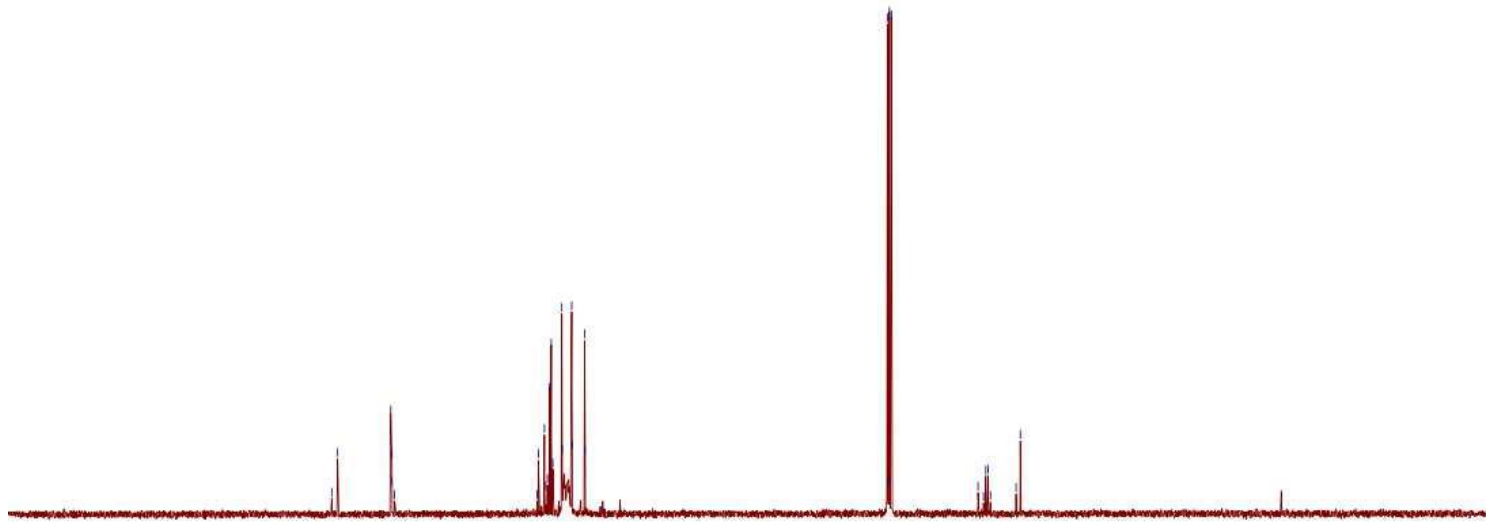

\begin{tabular}{|llllllllllllllllllllllllll}
210 & 200 & 190 & 180 & 170 & 160 & 150 & 140 & 130 & 120 & 110 & 100 & 90 & 80 & 70 & 60 & 50 & 40 & 30 & 20 & 10 & 0 & -10 & 10
\end{tabular}


${ }^{1} \mathrm{H}$ NMR of $4 \mathbf{d}\left(300 \mathrm{MHz}, \mathrm{CDCl}_{3}\right)$

GAR-4.2.10.fid

ResearchGroup Melchiorre

ICIQ_1H20p8s CDCl3 \{C: Bruker|TopSpin3.1\} gmelchiorre3 3

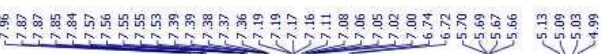<smiles>O=C(OCc1ccccc1)C(Cc1ccccc1)[n+]1c(-c2ccccc2)cc(-c2ccccc2)cc1-c1ccccc1</smiles>

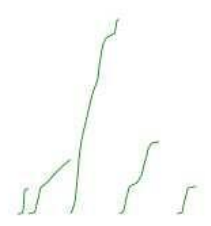

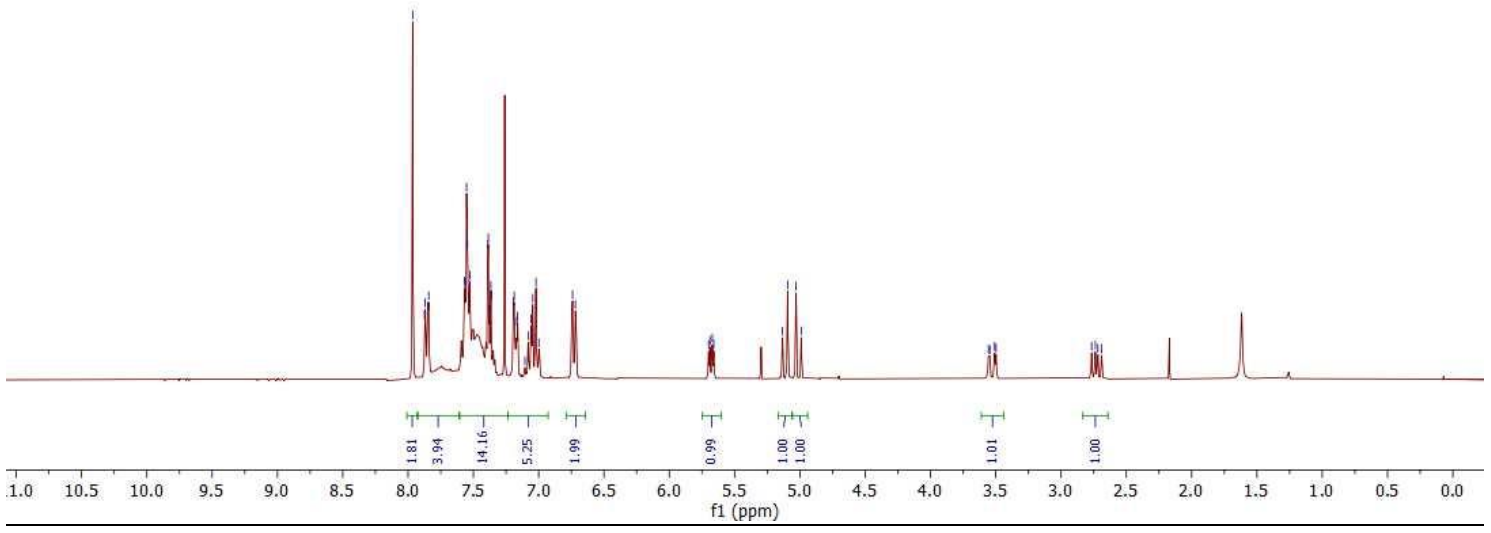

${ }^{13} \mathrm{C} \mathrm{NMR}$ of $\mathbf{4 d}\left(126 \mathrm{MHz}, \mathrm{CDCl}_{3}\right)$

GAR-4.2-C13.10.fid

ICIQ $13 \mathrm{C}\{1 \mathrm{H}\} 512 \mathrm{STBFO} \mathrm{CDCl}\{$ \{C: $\mid$ Bruker TOPSPIN $\}$ gmelchiorre3 20

$\begin{array}{lll}1 & 1 \\ 0 & 5 & 0\end{array}$

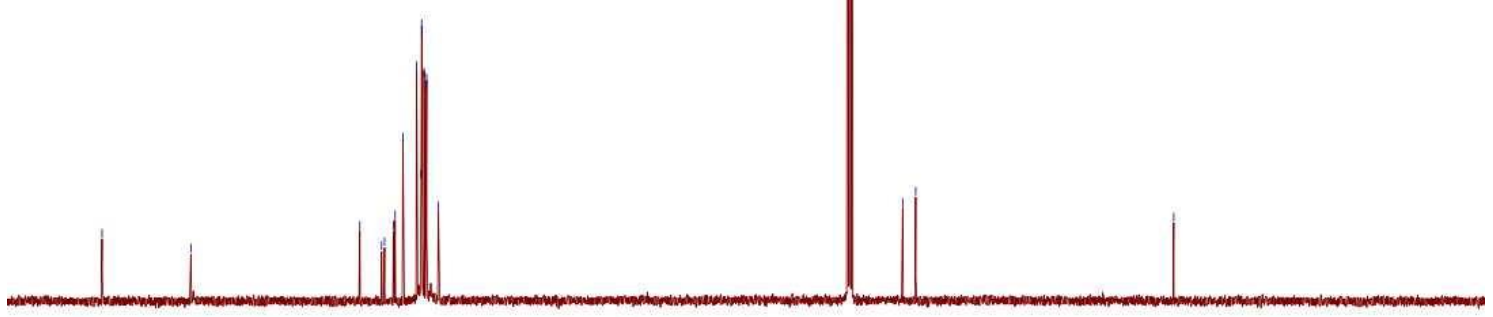

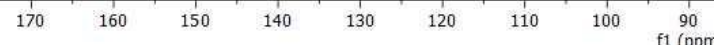

$\begin{array}{llllllll}1 & 1 & 1 & 1 & 1 & 1 & 1 & 1\end{array}$ 
${ }^{1} \mathrm{H} \mathrm{NMR}$ of $4 \mathbf{e}\left(300 \mathrm{MHz}, \mathrm{CDCl}_{3}\right)$

GAR-5.2.10.fid

ResearchGroup Melchiorre

ICIQ_1H20p8s CDCl3 \{C: Bruker|TopSpin3.1\} gmelchiorre3 6
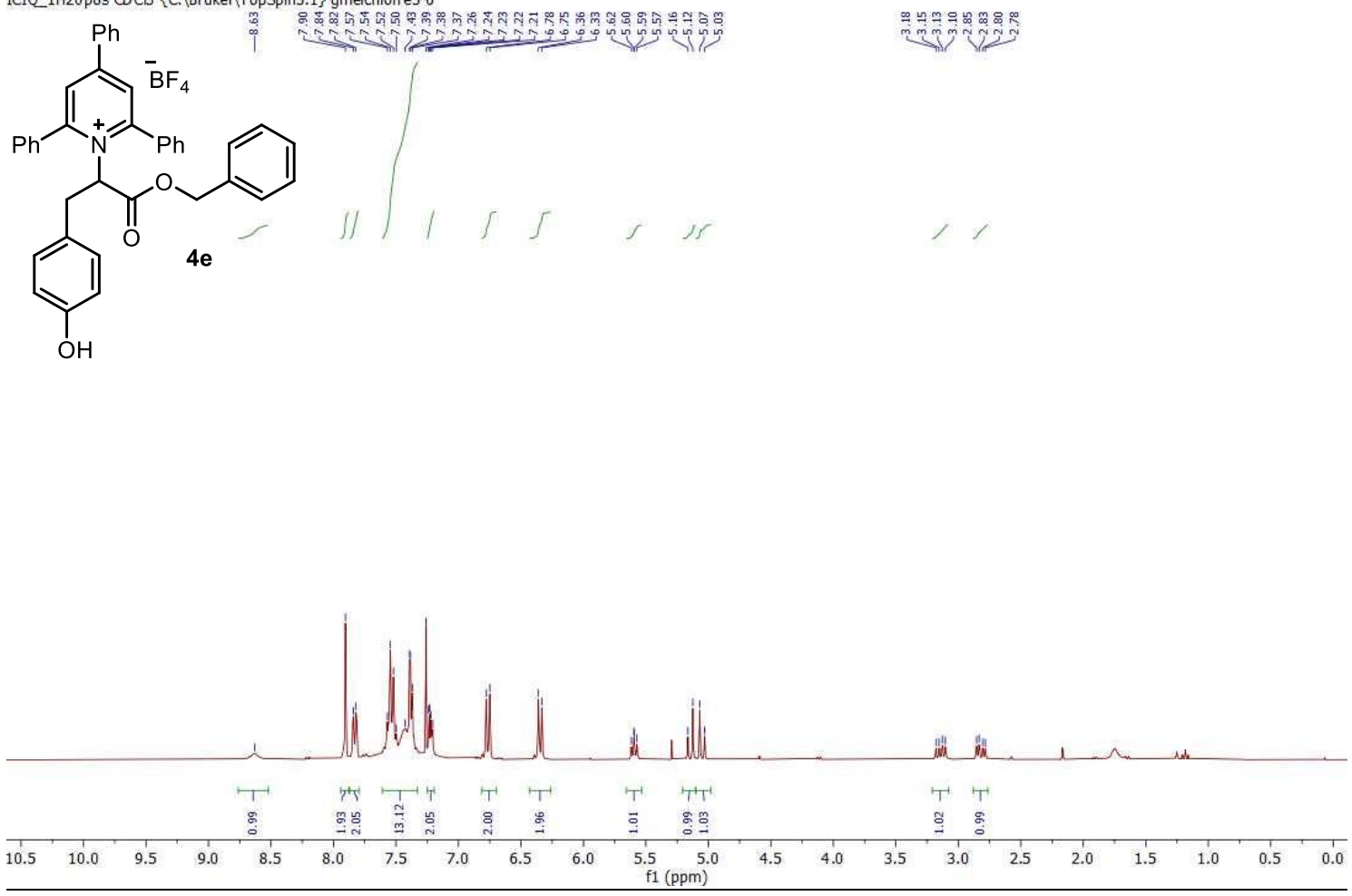

${ }^{13} \mathrm{C}$ NMR of $4 \mathbf{e}\left(126 \mathrm{MHz}, \mathrm{CDCl}_{3}\right)$

GAR-5-C13.10.fid

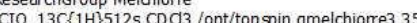
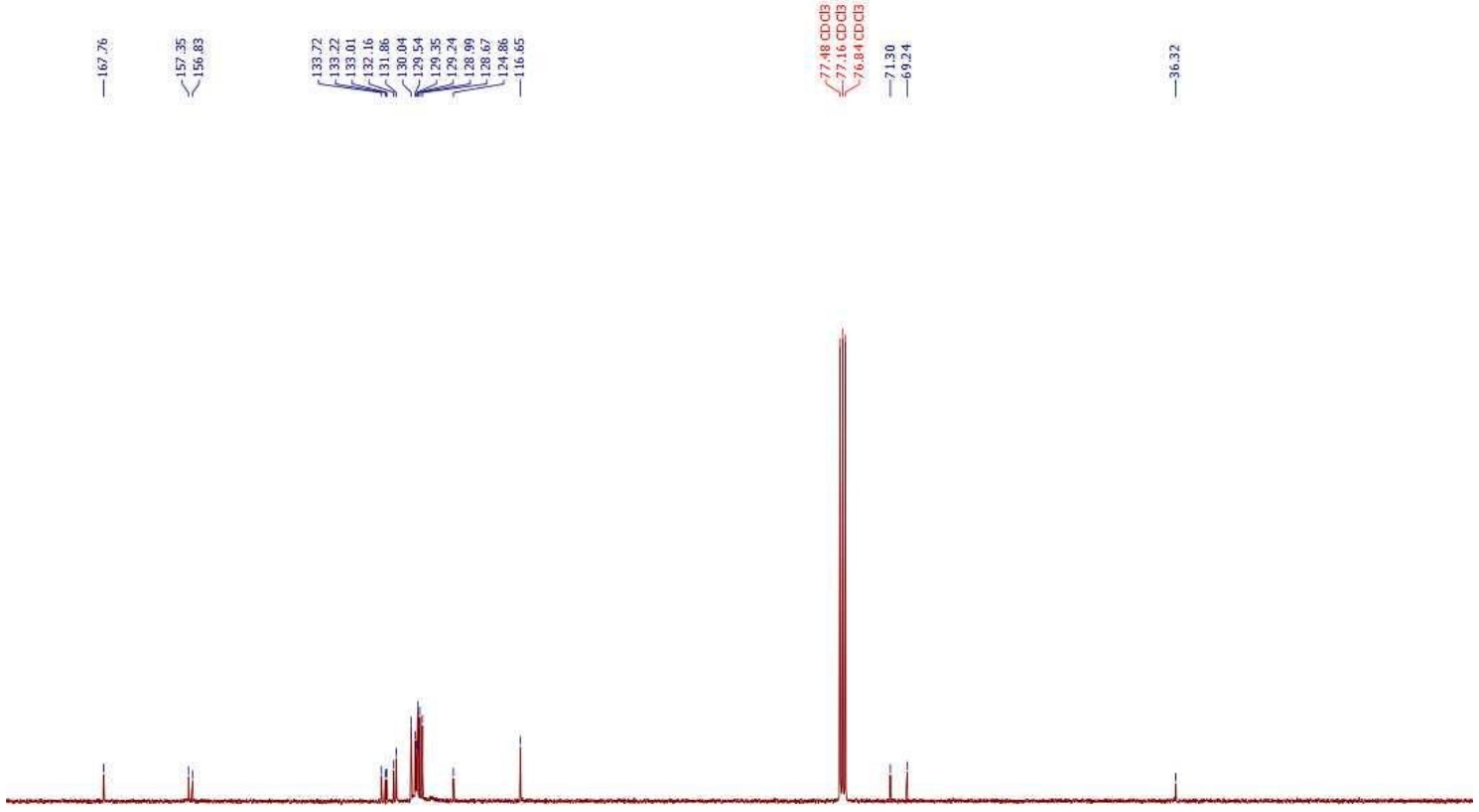

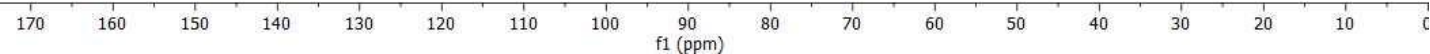


${ }^{1} \mathrm{H} \mathrm{NMR}$ of $\mathbf{4 f}\left(300 \mathrm{MHz}, \mathrm{CDCl}_{3}\right)$

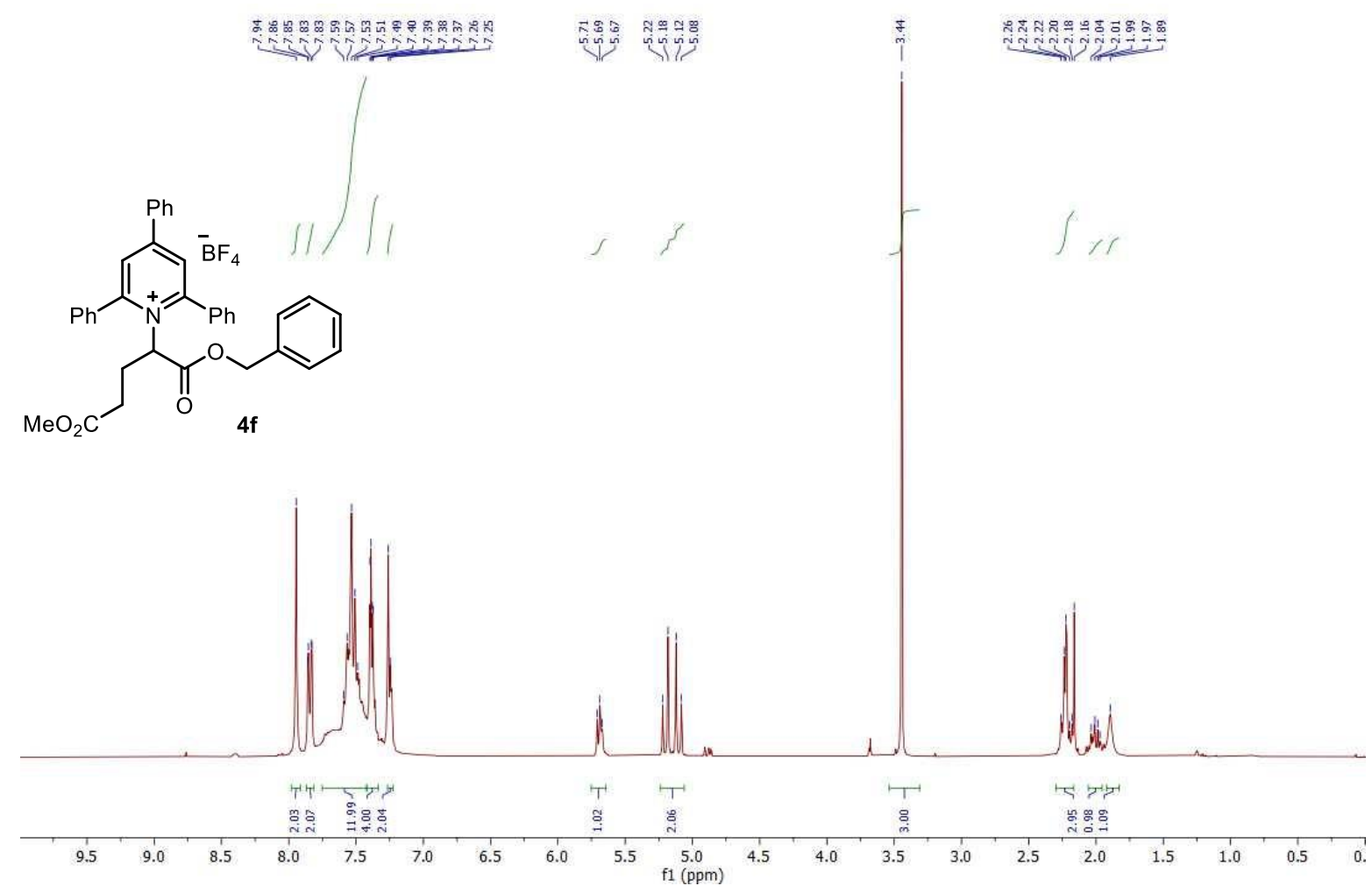

${ }^{13} \mathrm{C}$ NMR of $4 \mathbf{f}\left(126 \mathrm{MHz}, \mathrm{CDCl}_{3}\right)$

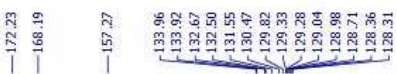
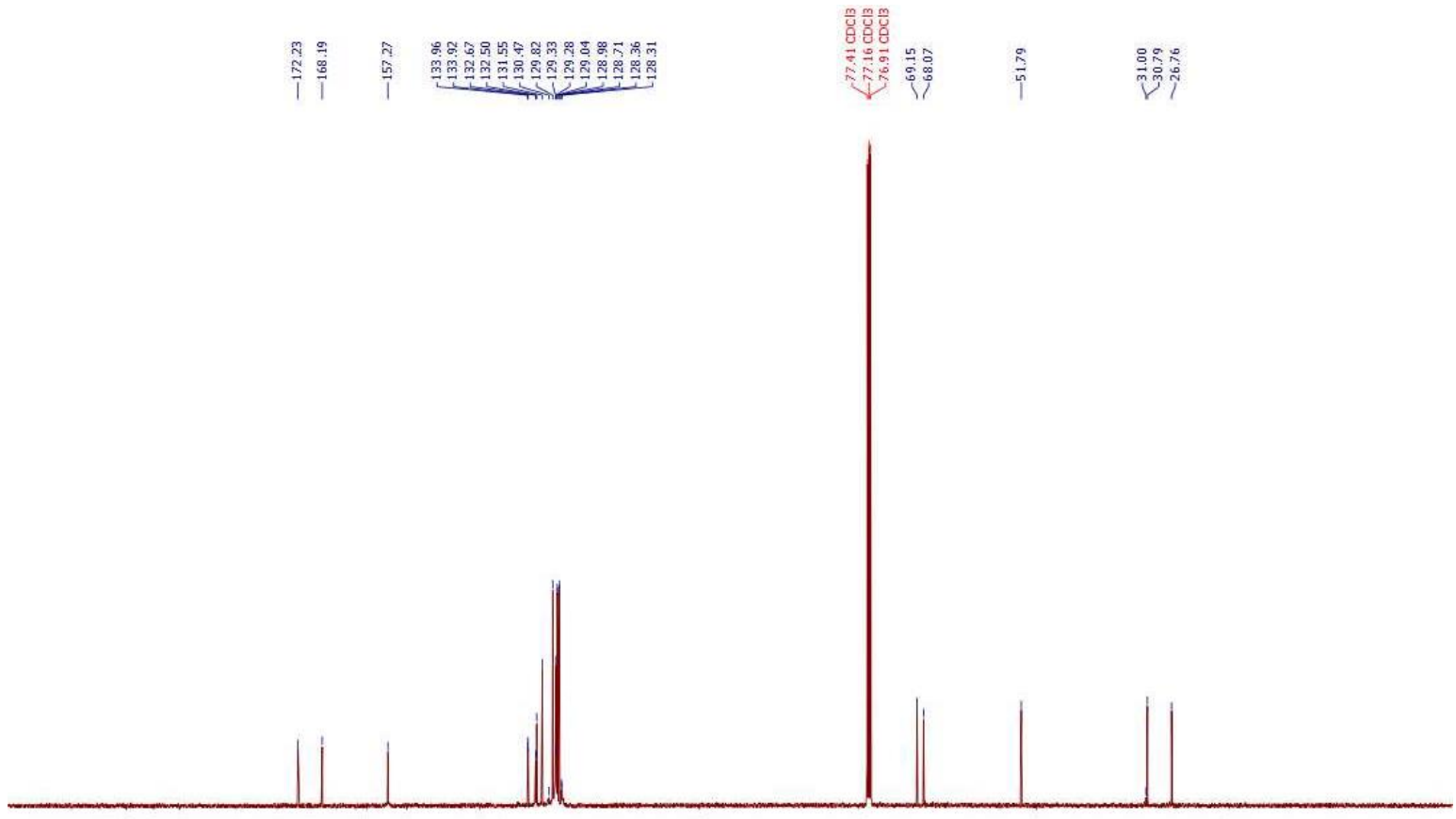

$\begin{array}{lllllllllllllllllllllllllllllllllll}1 & 210 & 200 & 190 & 180 & 170 & 160 & 150 & 140 & 130 & 120 & 110 & 100 & 90 & 80 & 70 & 60 & 50 & 40 & 30 & 20 & 10 & 0 & -10 & -2\end{array}$ 
${ }^{1} \mathrm{H}$ NMR of $\mathbf{4 g}\left(300 \mathrm{MHz}, \mathrm{CDCl}_{3}\right)$<smiles></smiles>

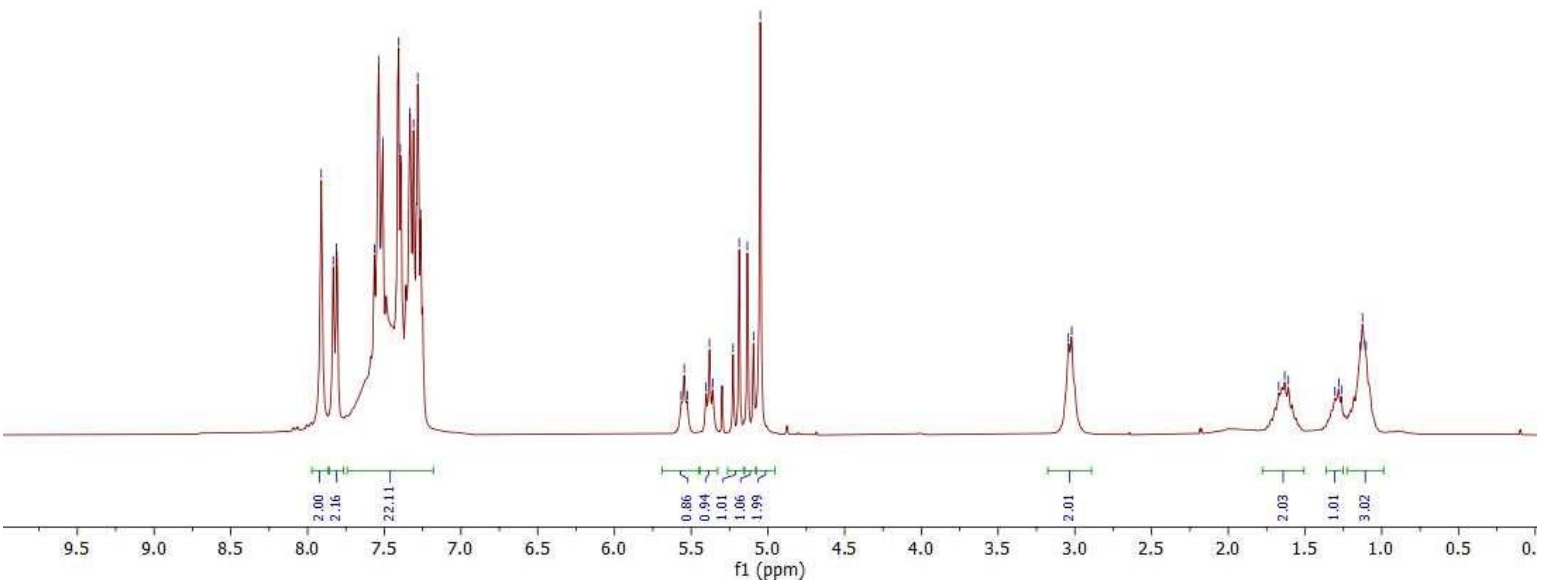

${ }^{13} \mathrm{C}$ NMR of $4 \mathbf{g}\left(126 \mathrm{MHz}, \mathrm{CDCl}_{3}\right)$

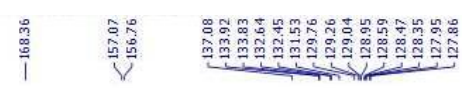

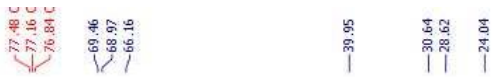

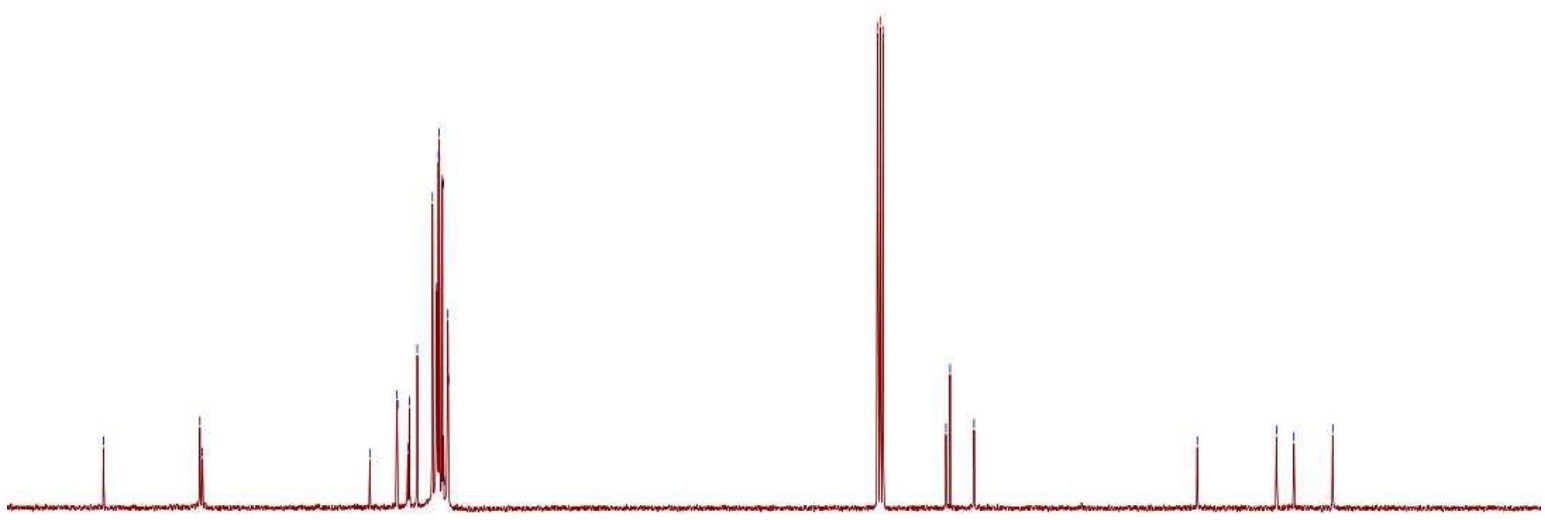


${ }^{1} \mathrm{H}$ NMR of 5a $\left(400 \mathrm{MHz}, \mathrm{CDCl}_{3}\right)$

BPL74-pure.10.fid ICIO 1 H12p8S MCIB /opt/topsoin biaroche 76

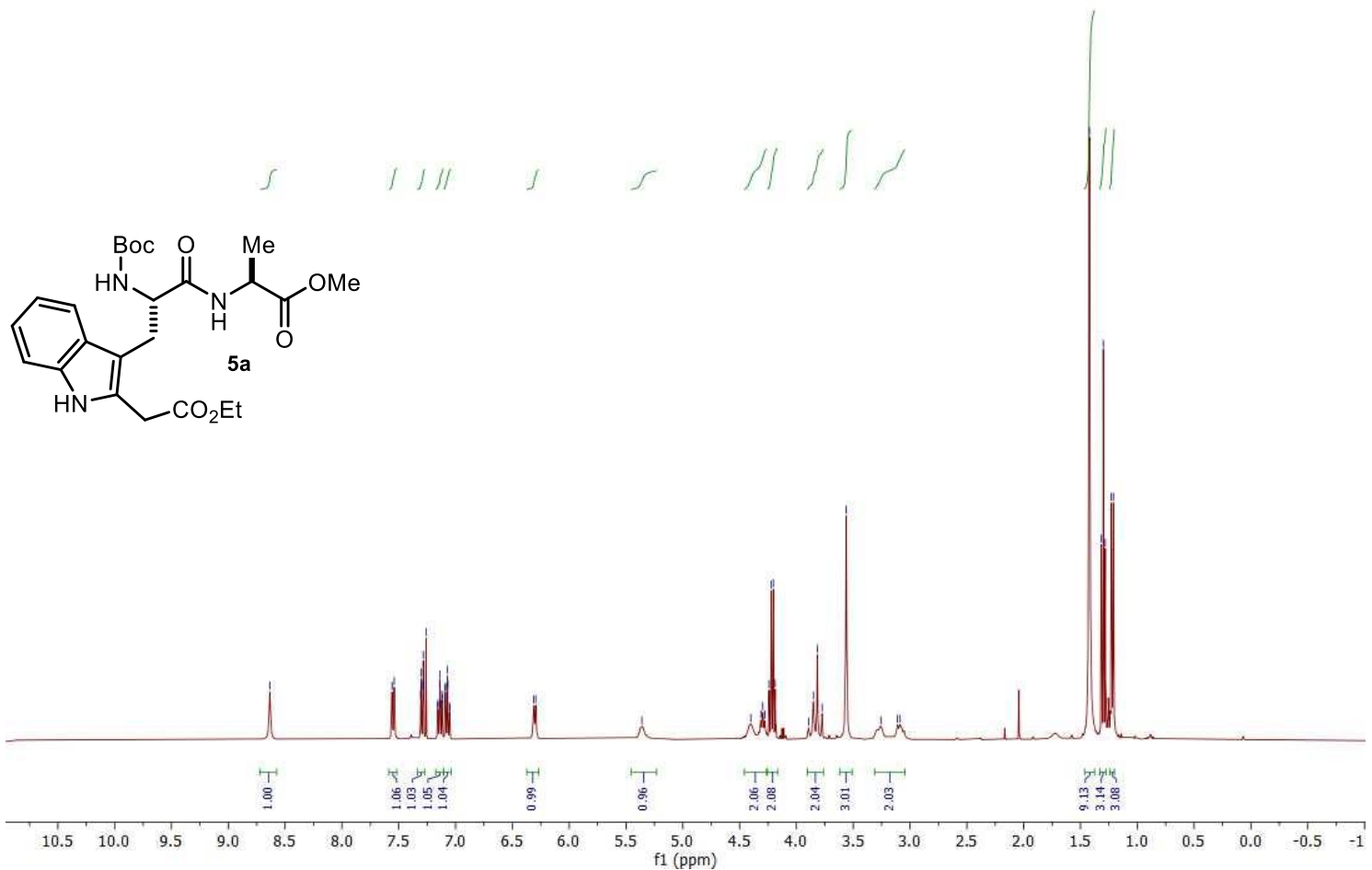

${ }^{13} \mathrm{C}$ NMR of 5a $\left(101 \mathrm{MHz}, \mathrm{CDCl}_{3}\right)$

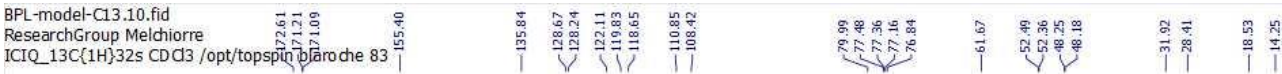

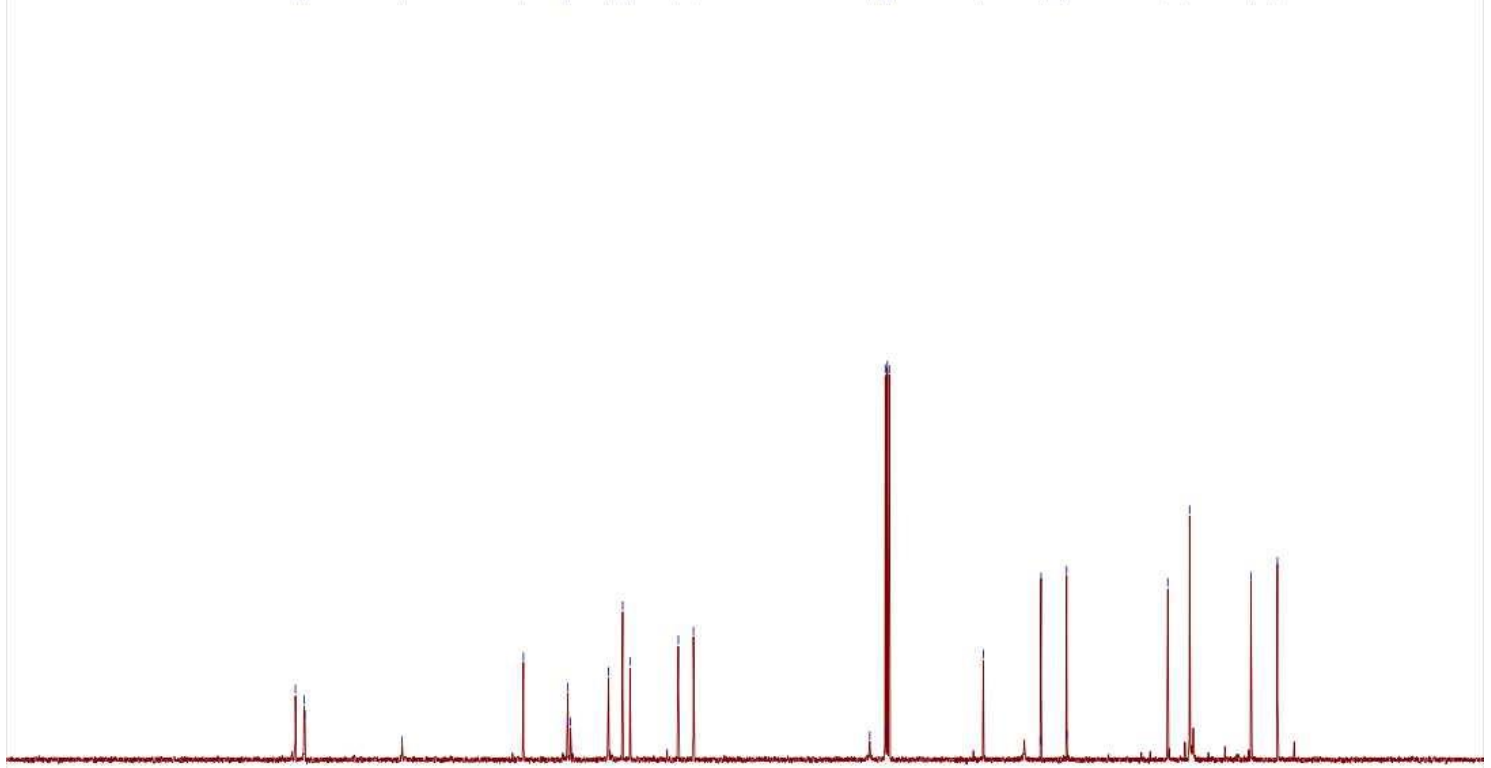

$\begin{array}{lllllllllllllllllllllll}1 & 1 \\ 210 & 200 & 190 & 180 & 170 & 160 & 150 & 140 & 130 & 120 & 110 & 100 & 90 & 80 & 70 & 60 & 50 & 40 & 30 & 20 & 10 & 0 & -10\end{array}$ 
${ }^{1} \mathrm{H}$ NMR of $\mathbf{5 b}\left(400 \mathrm{MHz}, \mathrm{CDCl}_{3}\right)$

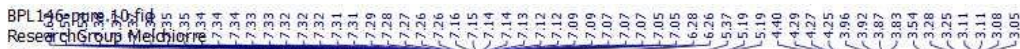

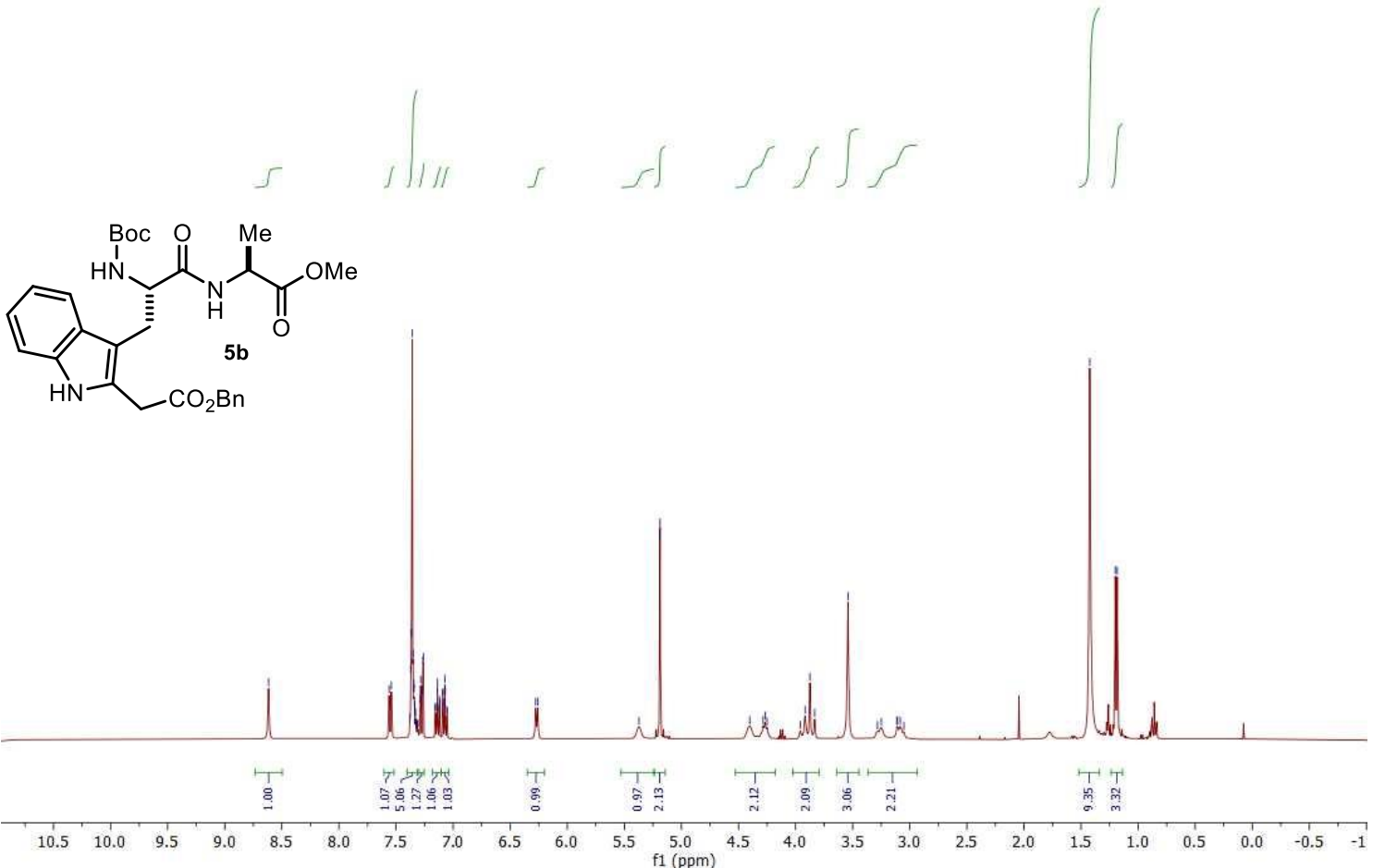

${ }^{13} \mathrm{C} \mathrm{NMR}$ of $\mathbf{5 b}\left(101 \mathrm{MHz}, \mathrm{CDCl}_{3}\right)$

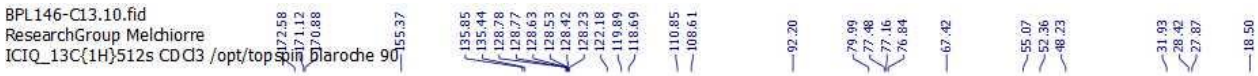

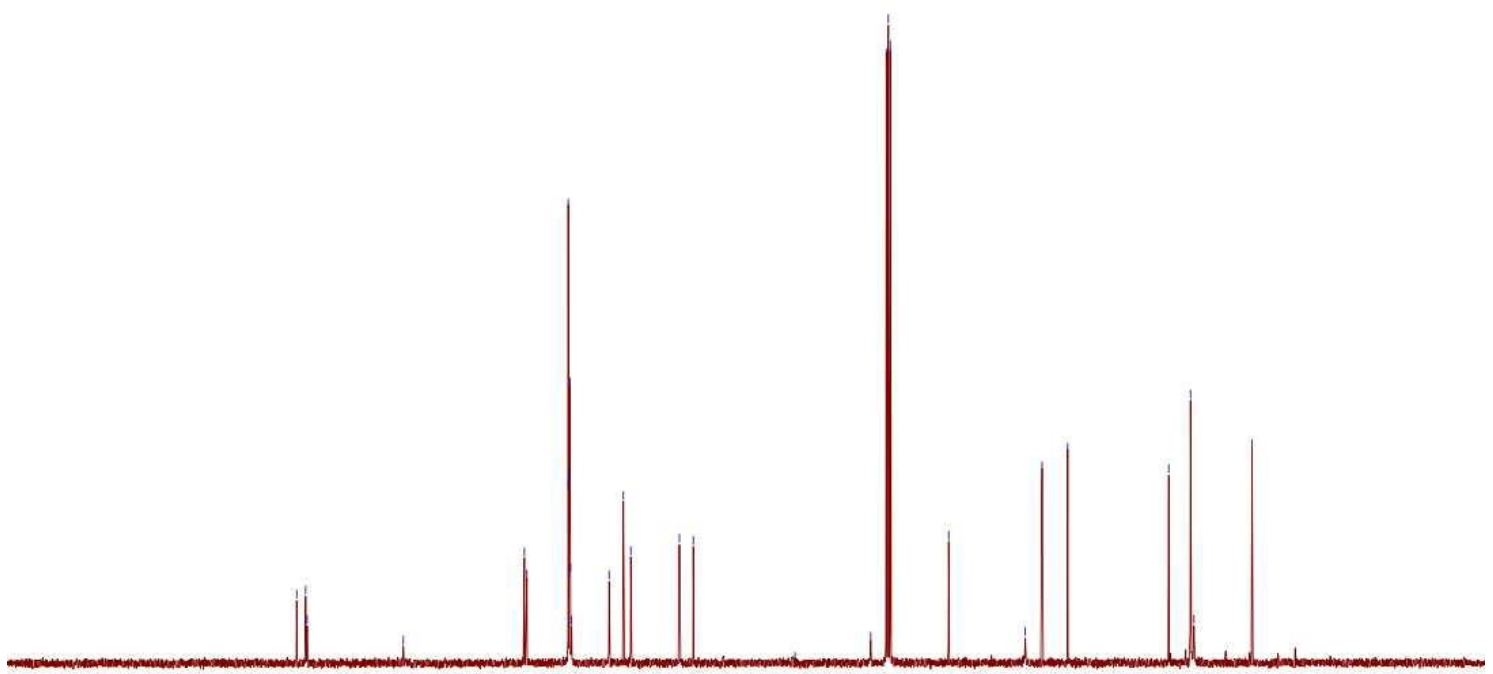

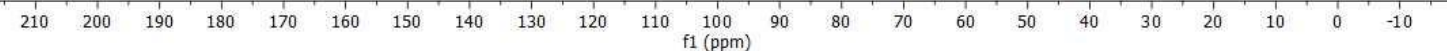


${ }^{1} \mathrm{H} \mathrm{NMR}$ of $\mathbf{5 c}\left(500 \mathrm{MHz}, \mathrm{CDCl}_{3}\right)$

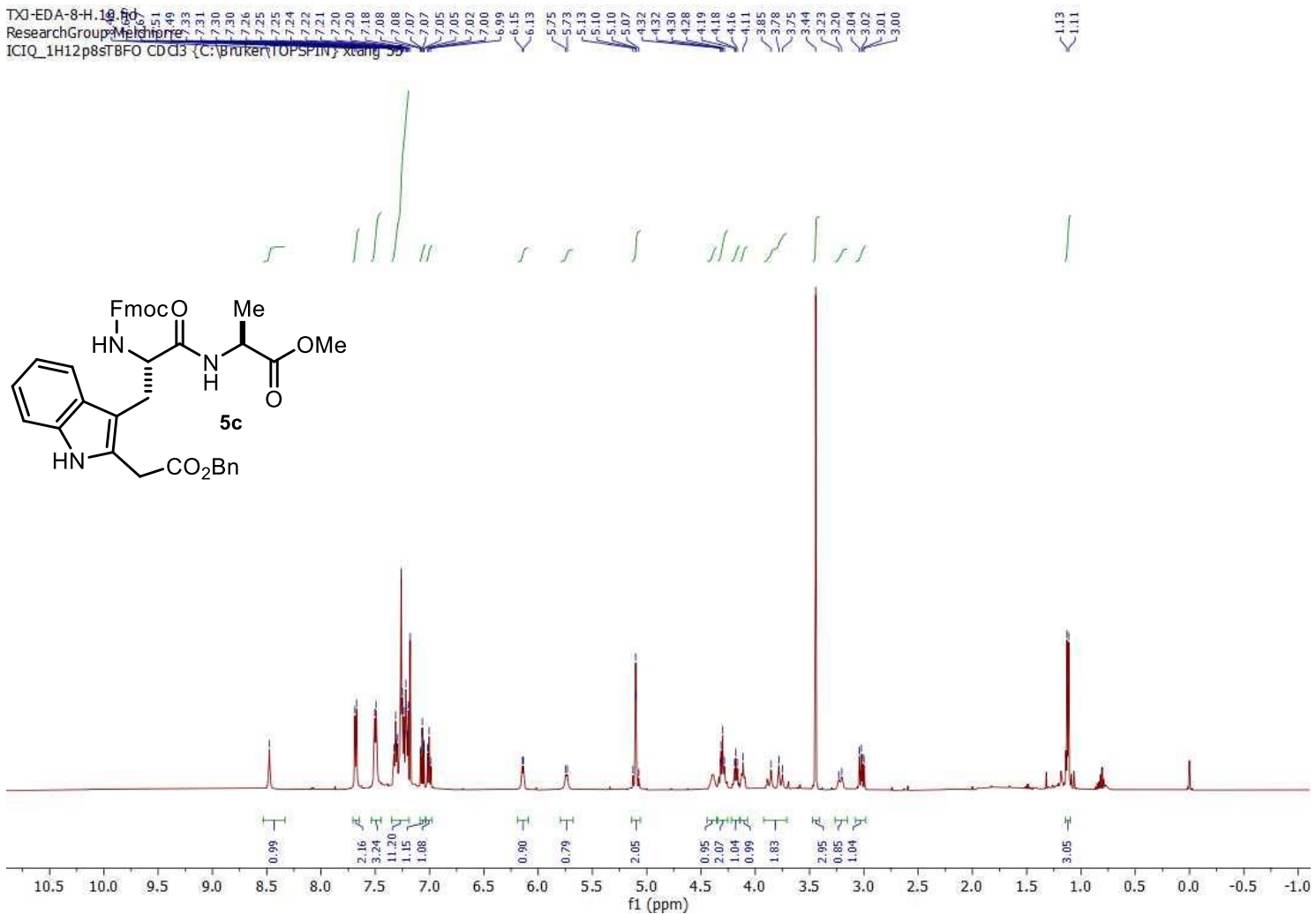

${ }^{13} \mathrm{C}$ NMR of $5 \mathbf{c}\left(126 \mathrm{MHz}, \mathrm{CDCl}_{3}\right)$

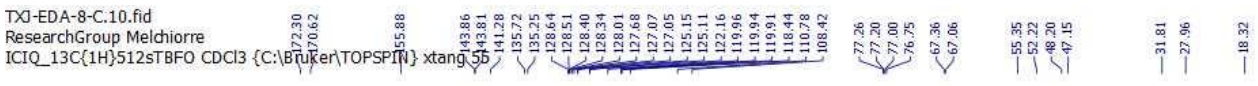

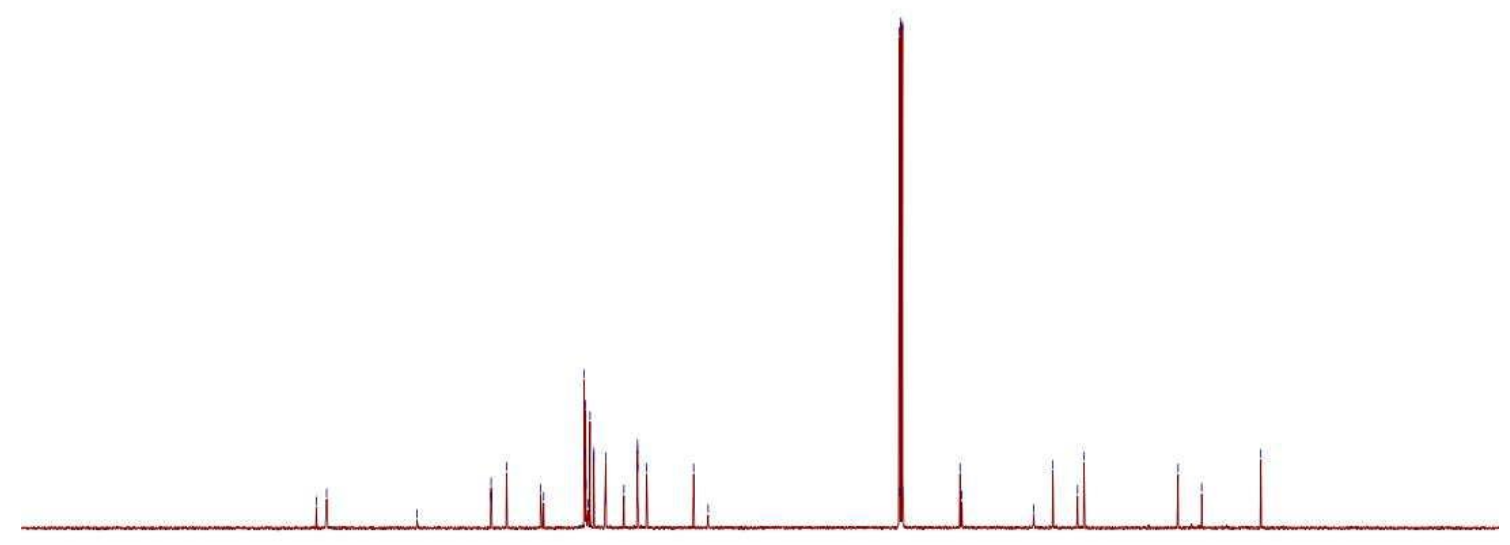

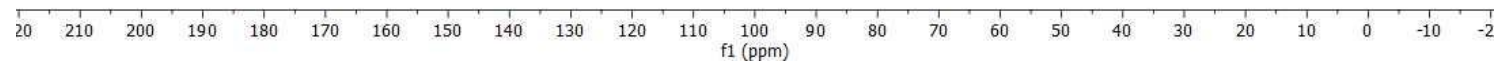


${ }^{1} \mathrm{H}$ NMR of $\mathbf{5 d}\left(500 \mathrm{MHz}, \mathrm{CDCl}_{3}\right)$

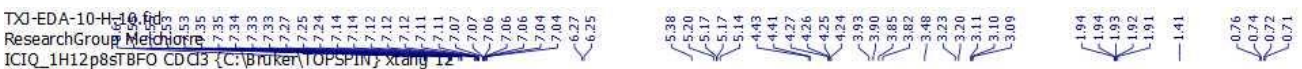

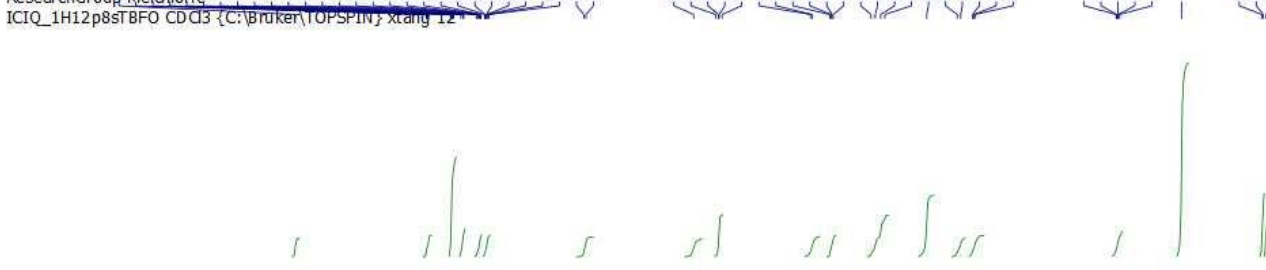<smiles>COC(=O)[C@H](NC(=O)[C@H](Cc1c(CC(=O)OCc2ccccc2)[nH]c2ccccc12)NC(=O)OCc1ccccc1)C(C)C</smiles>

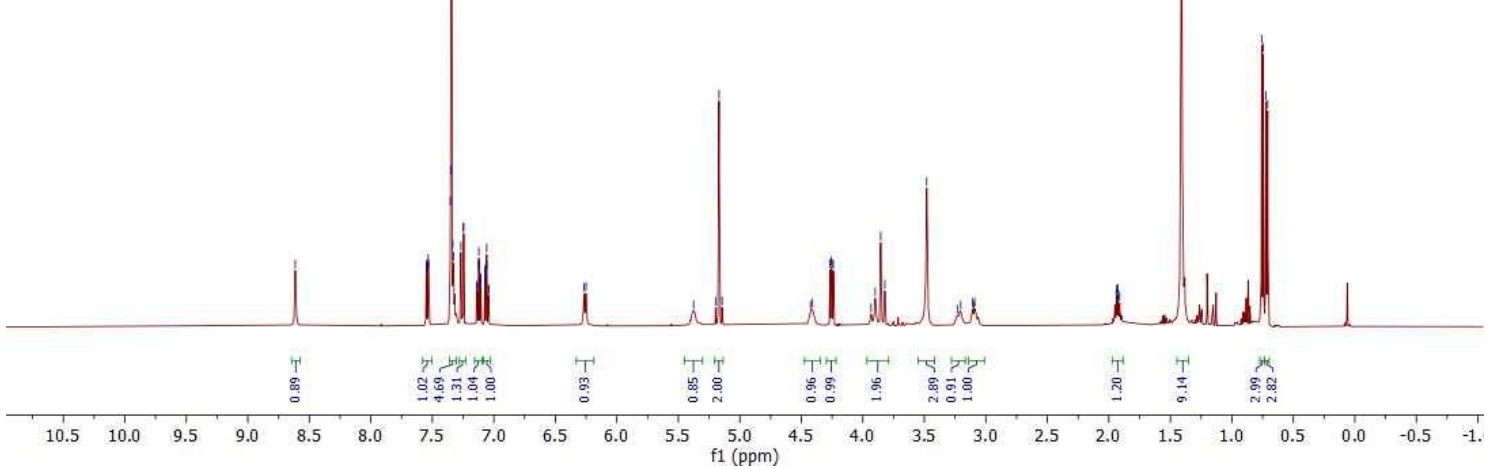

${ }^{13} \mathrm{C}$ NMR of $\mathbf{5 d}\left(126 \mathrm{MHz}, \mathrm{CDCl}_{3}\right)$

TX-EDA-10-H.11.fid

욕 ำ

PSPIN̂̉, xtang 12

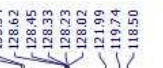

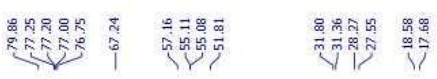

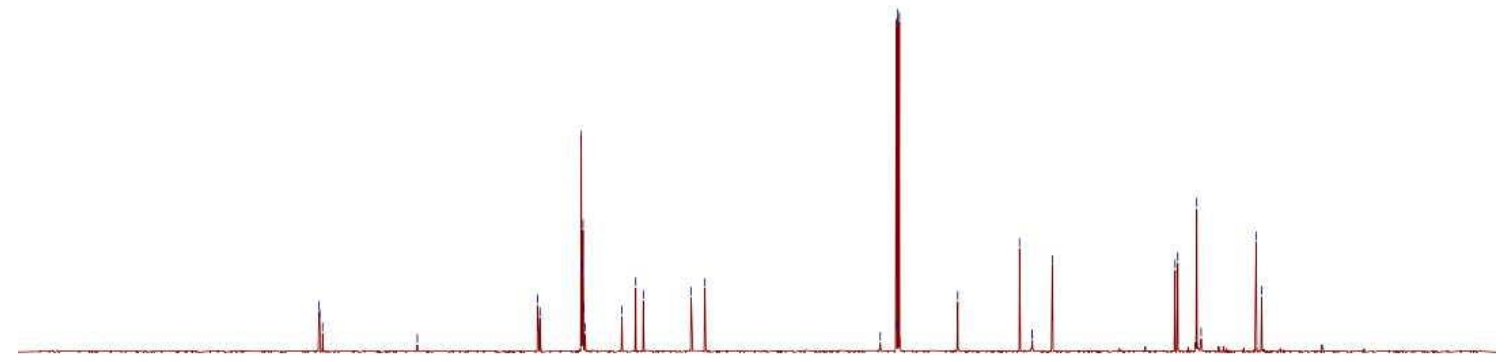

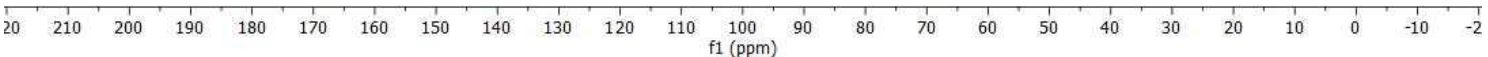


${ }^{1} \mathrm{H} \mathrm{NMR}$ of $\mathbf{5 e}\left(500 \mathrm{MHz}, \mathrm{CDCl}_{3}\right)$

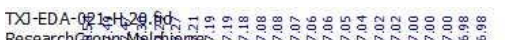

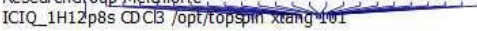

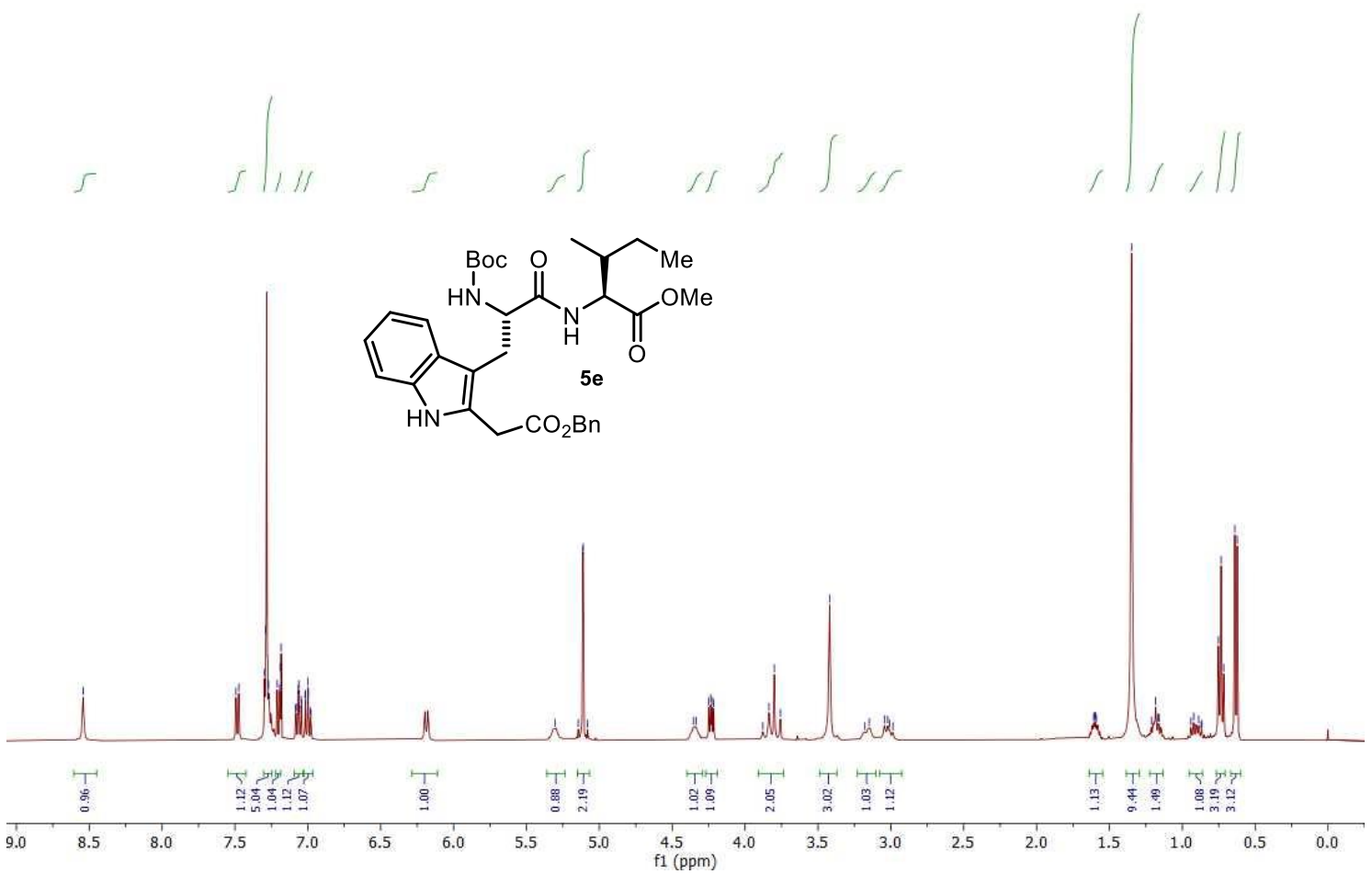

${ }^{13} \mathrm{C}$ NMR of $\mathbf{5 e}\left(126 \mathrm{MHz}, \mathrm{CDCl}_{3}\right)$

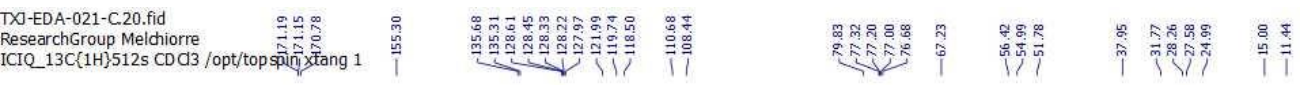

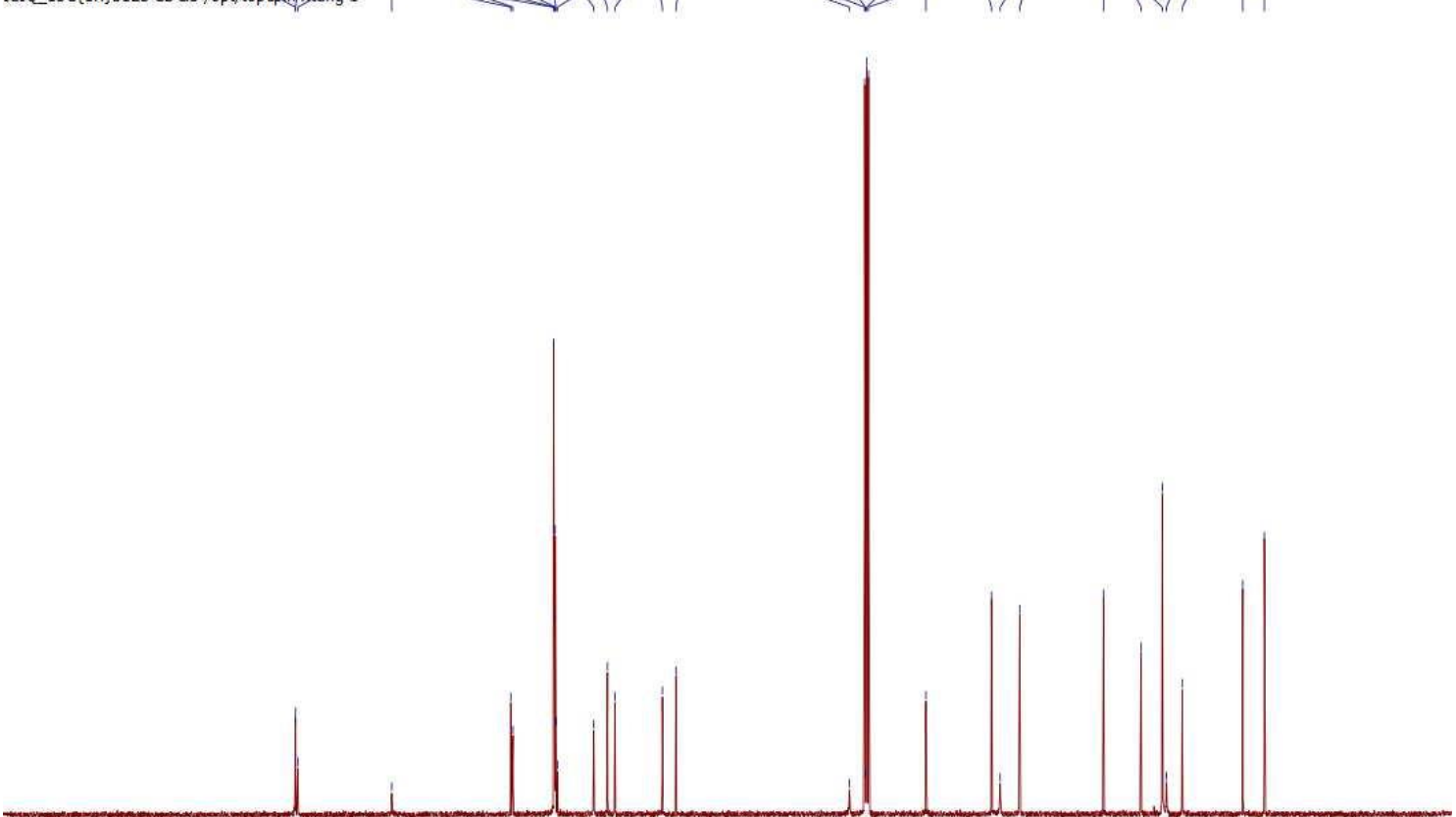

$\begin{array}{llllllllllllllllllllllllllll}210 & 200 & 190 & 180 & 170 & 160 & 150 & 140 & 130 & 120 & 110 & 100 & 90 & 80 & 70 & 60 & 50 & 40 & 30 & 20 & 10 & 0 & -10\end{array}$ 
${ }^{1} \mathrm{H}$ NMR of $\mathbf{5 f}\left(400 \mathrm{MHz}, \mathrm{CDCl}_{3}\right)$

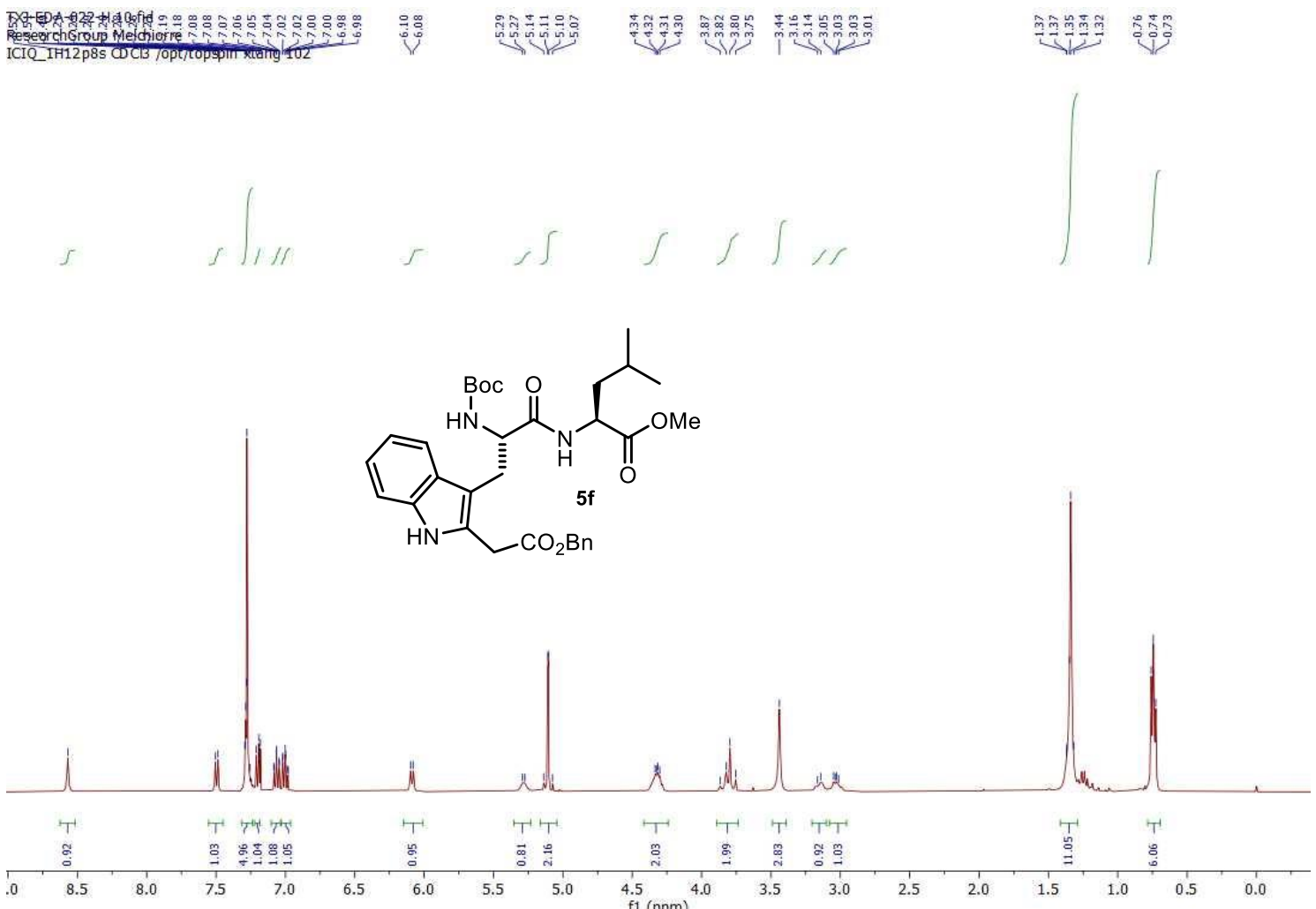

${ }^{13} \mathrm{C}$ NMR of $\mathbf{5 f}\left(101 \mathrm{MHz}, \mathrm{CDCl}_{3}\right)$

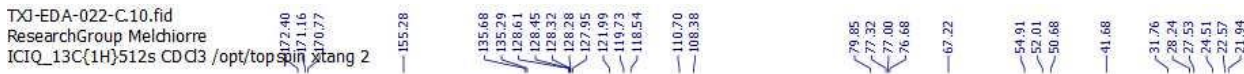

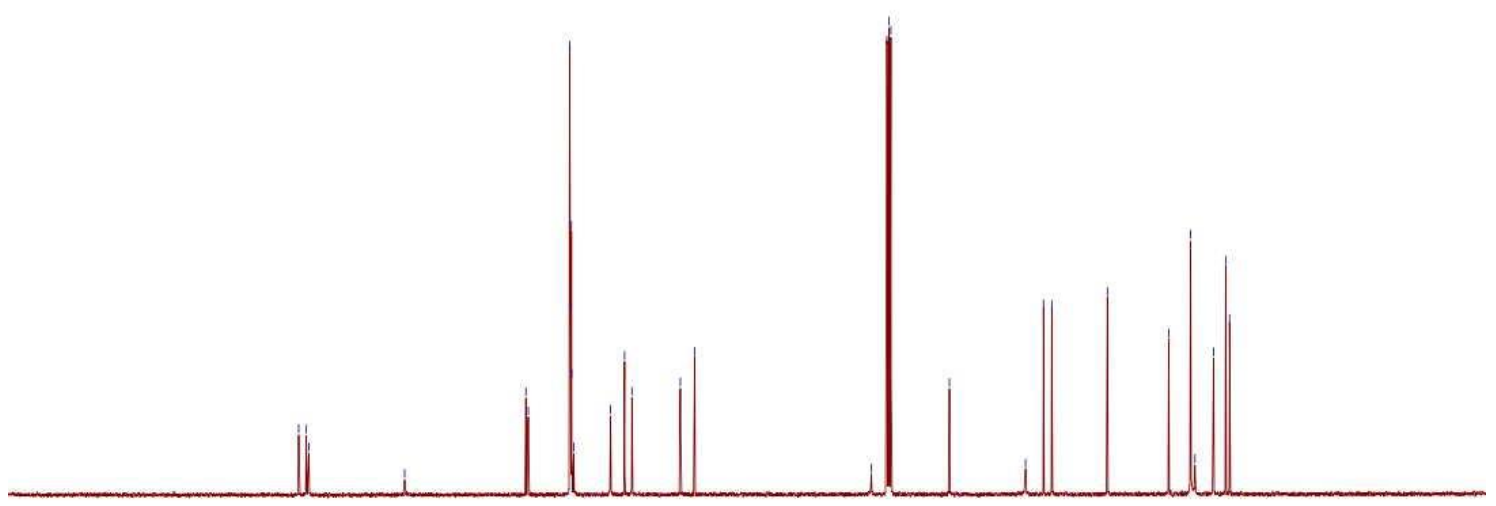

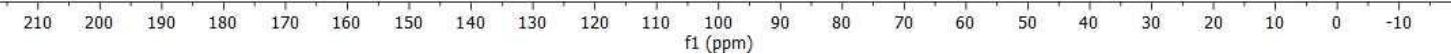


${ }^{1} \mathrm{H} \mathrm{NMR}$ of $\mathbf{5 g}\left(500 \mathrm{MHz}, \mathrm{CDCl}_{3}\right)$

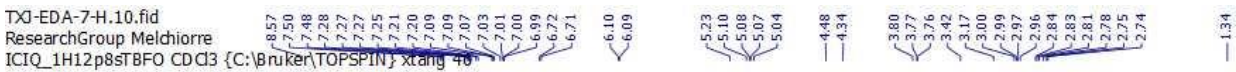

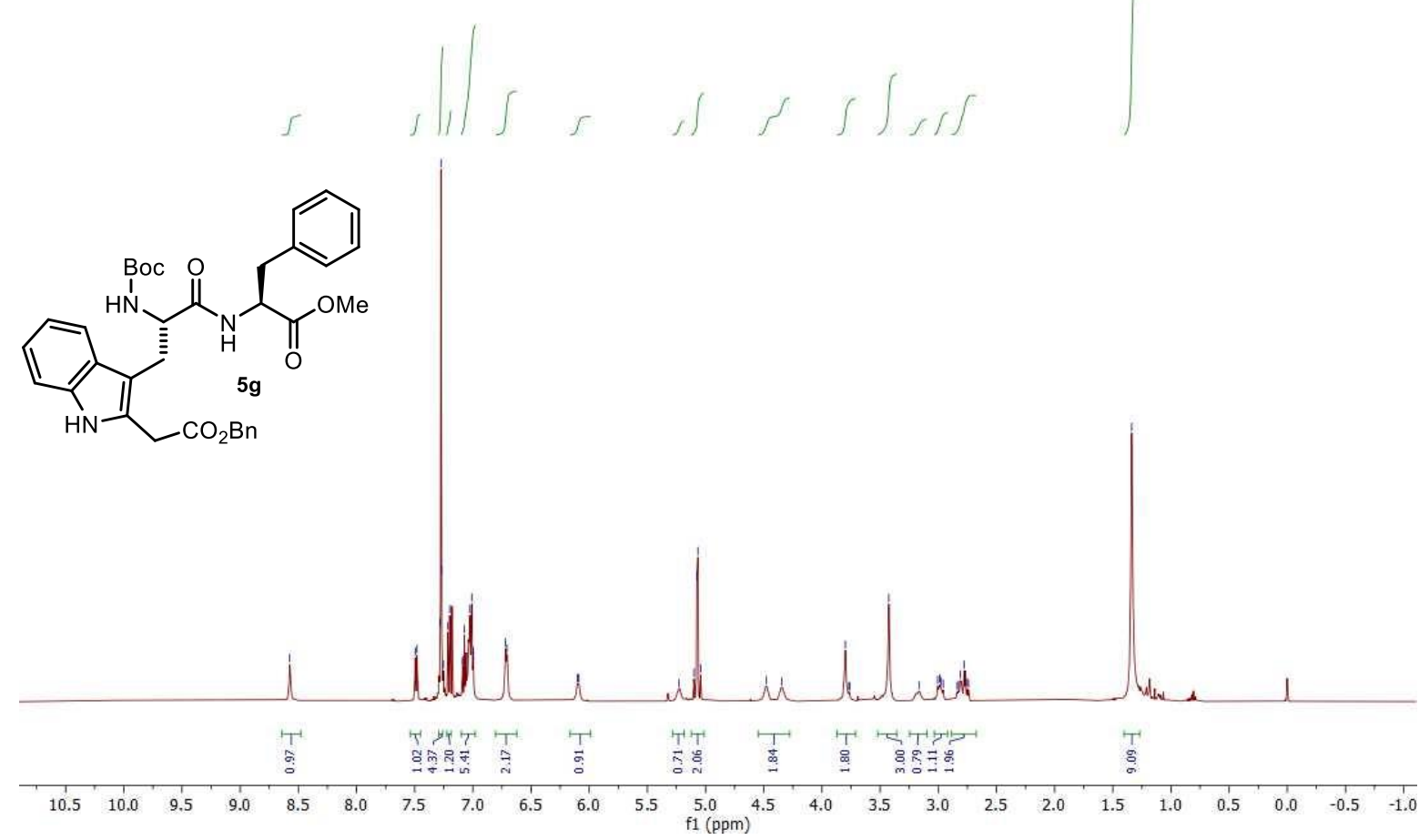

${ }^{13} \mathrm{C}$ NMR of $\mathbf{5 g}\left(126 \mathrm{MHz}, \mathrm{CDCl}_{3}\right)$

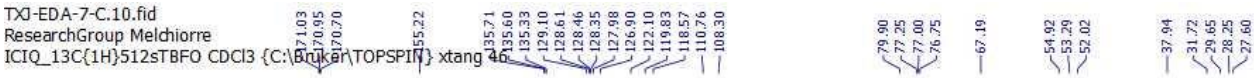

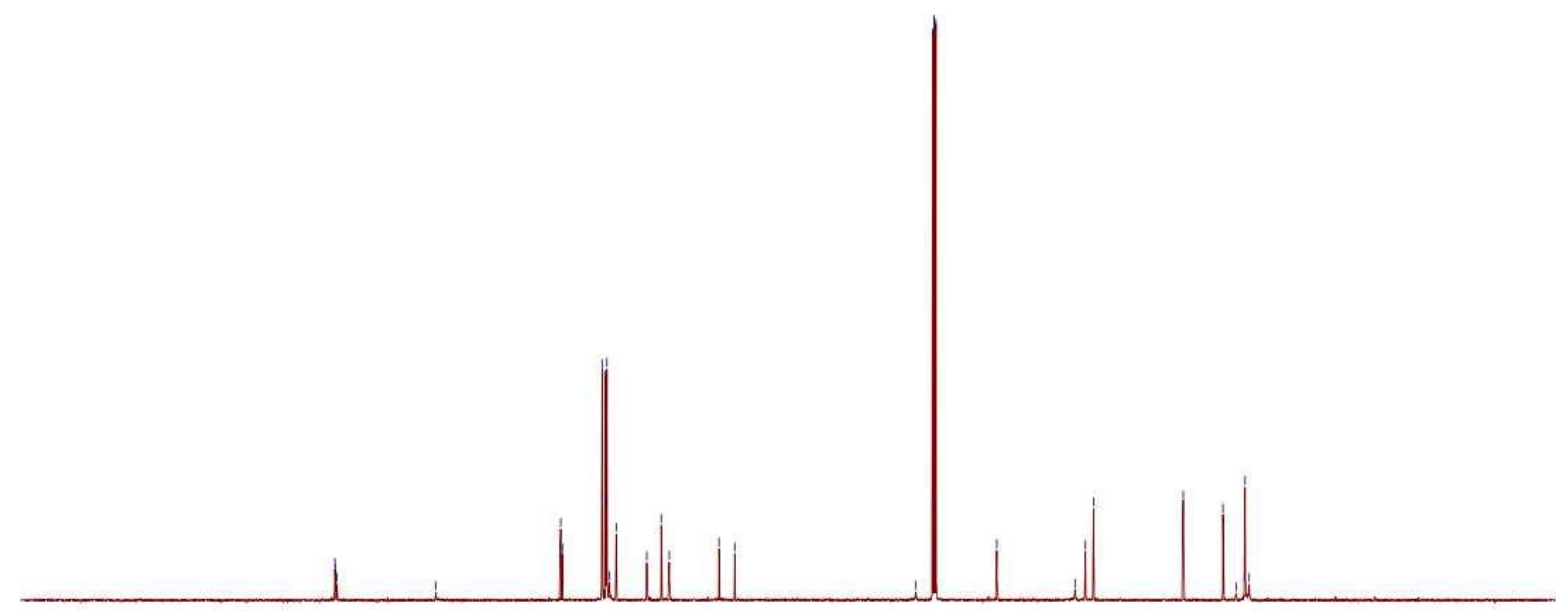

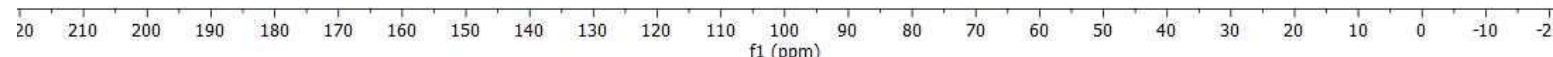


${ }^{1} \mathrm{H}$ NMR of $\mathbf{5 h}\left(500 \mathrm{MHz}, \mathrm{CDCl}_{3}\right)$

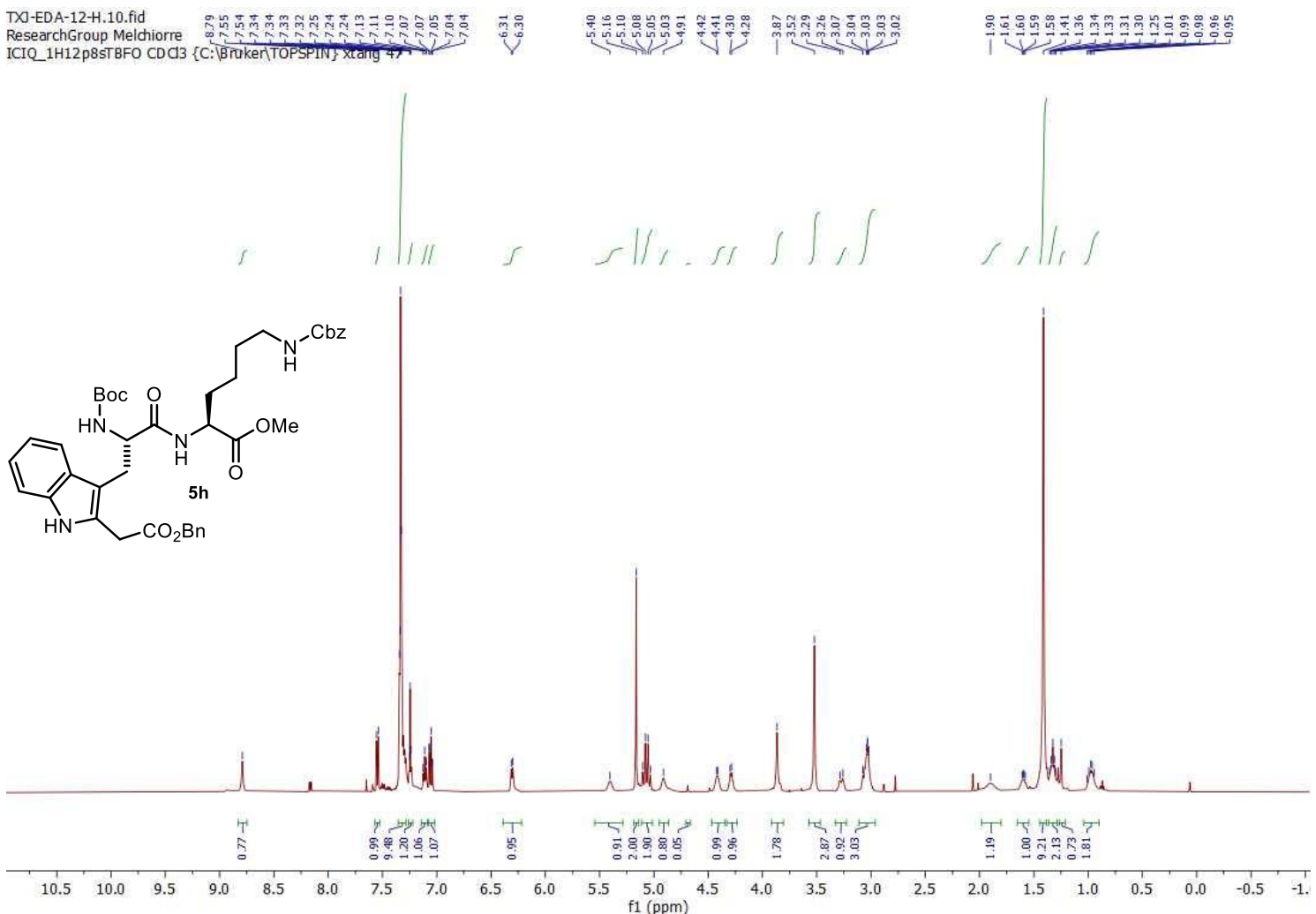

${ }^{13} \mathrm{C}$ NMR of $\mathbf{5 h}\left(126 \mathrm{MHz}, \mathrm{CDCl}_{3}\right)$

TX-EDA-12-C.10.fid

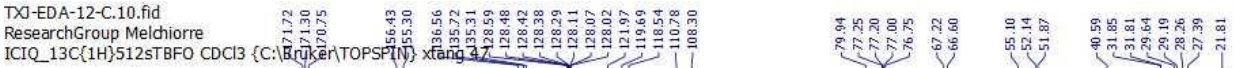

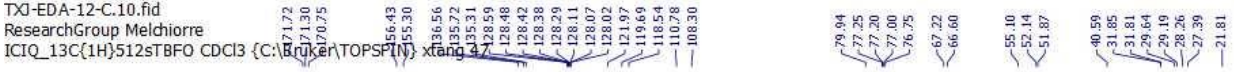

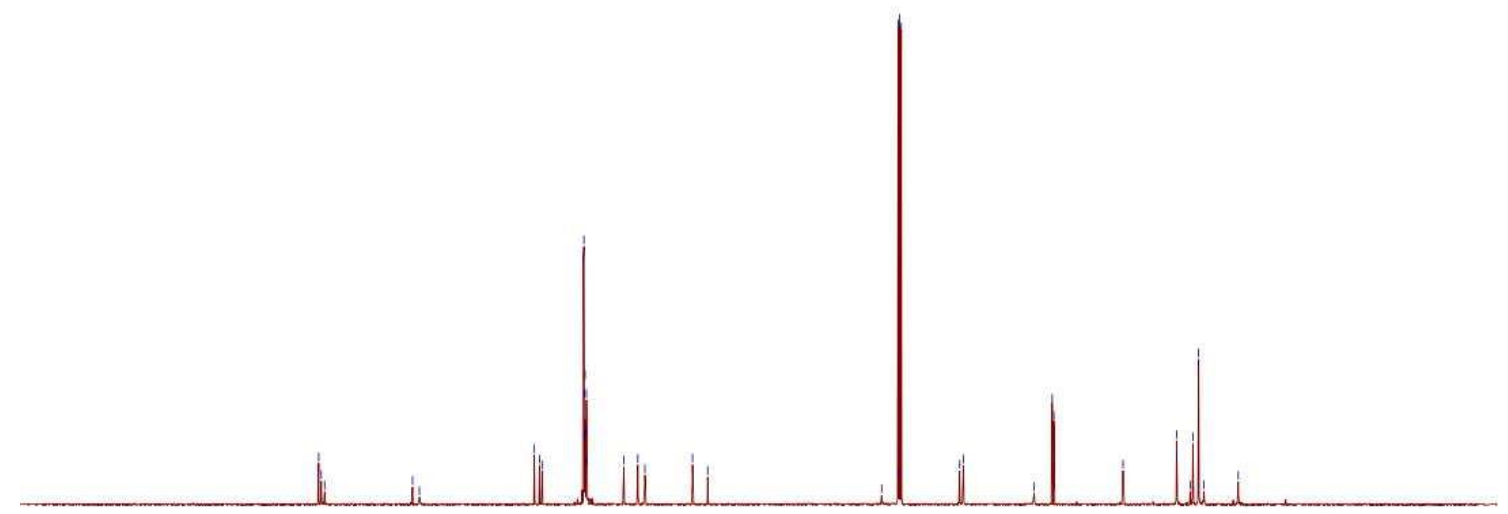

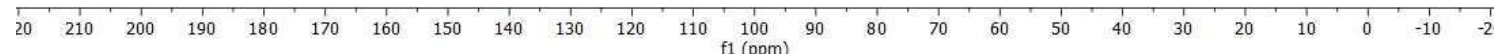


${ }^{1} \mathrm{H}$ NMR of $\mathbf{5 i}\left(400 \mathrm{MHz}, \mathrm{CDCl}_{3}\right.$ )

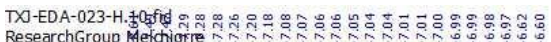

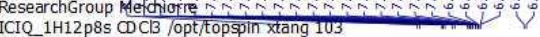

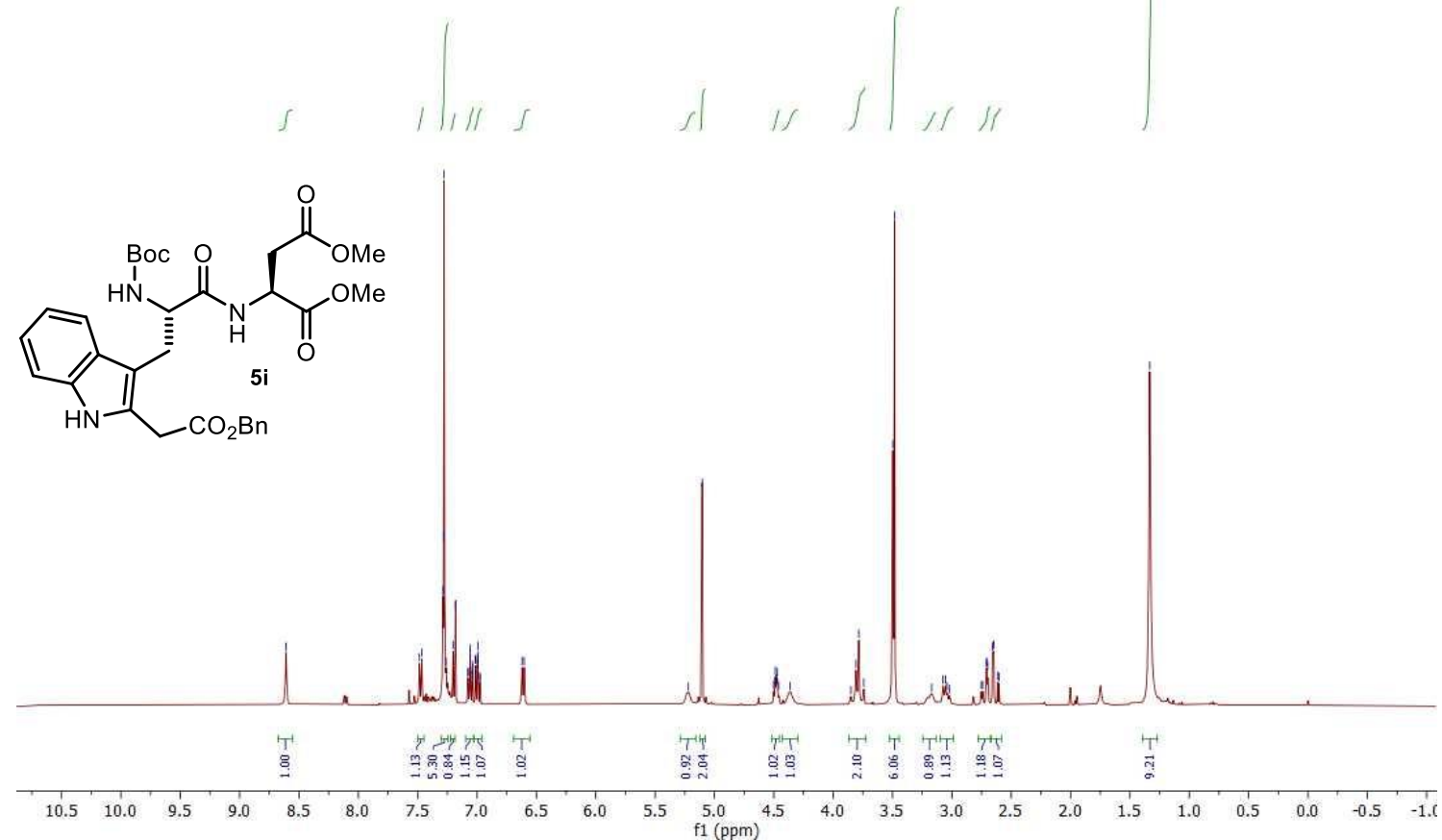

${ }^{13} \mathrm{C}$ NMR of $5 \mathbf{i}\left(101 \mathrm{MHz}, \mathrm{CDCl}_{3}\right)$

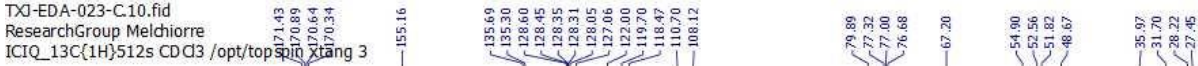

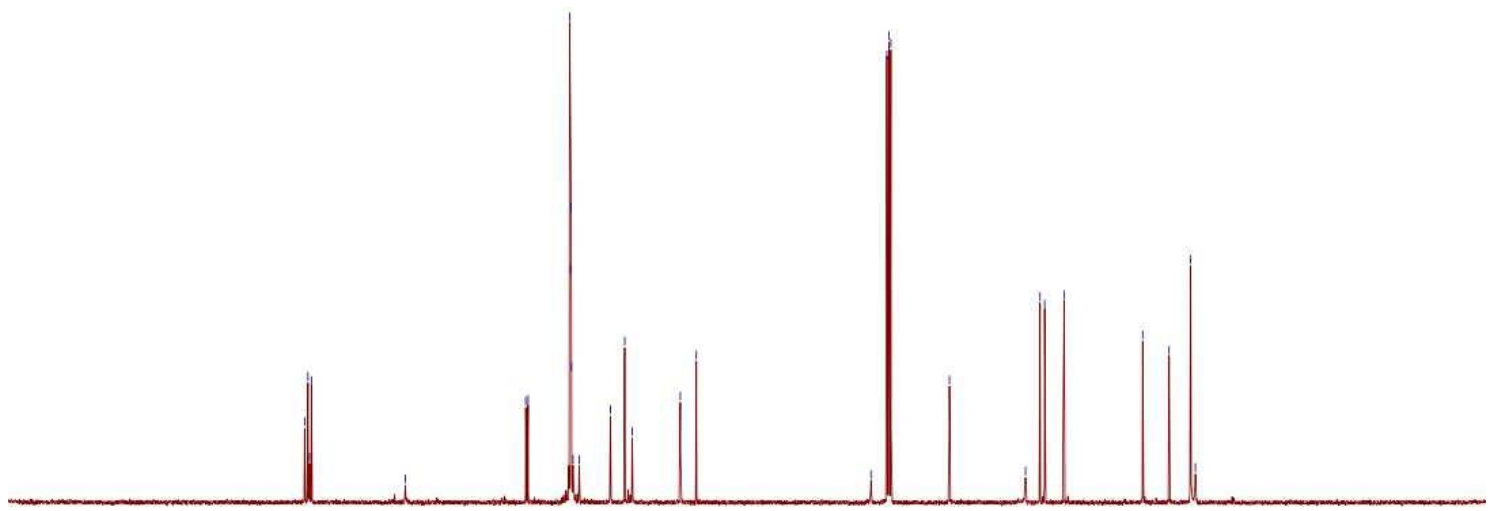

$\begin{array}{lllllllllllllllllllllll}210 & 200 & 190 & 180 & 170 & 160 & 150 & 140 & 130 & 120 & 110 & 100 & 90 & 80 & 70 & 60 & 50 & 40 & 30 & 20 & 10 & 0 & -10\end{array}$ 
${ }^{1} \mathrm{H} \mathrm{NMR}$ of $\mathbf{5 j}\left(400 \mathrm{MHz}, \mathrm{CDCl}_{3}\right)$
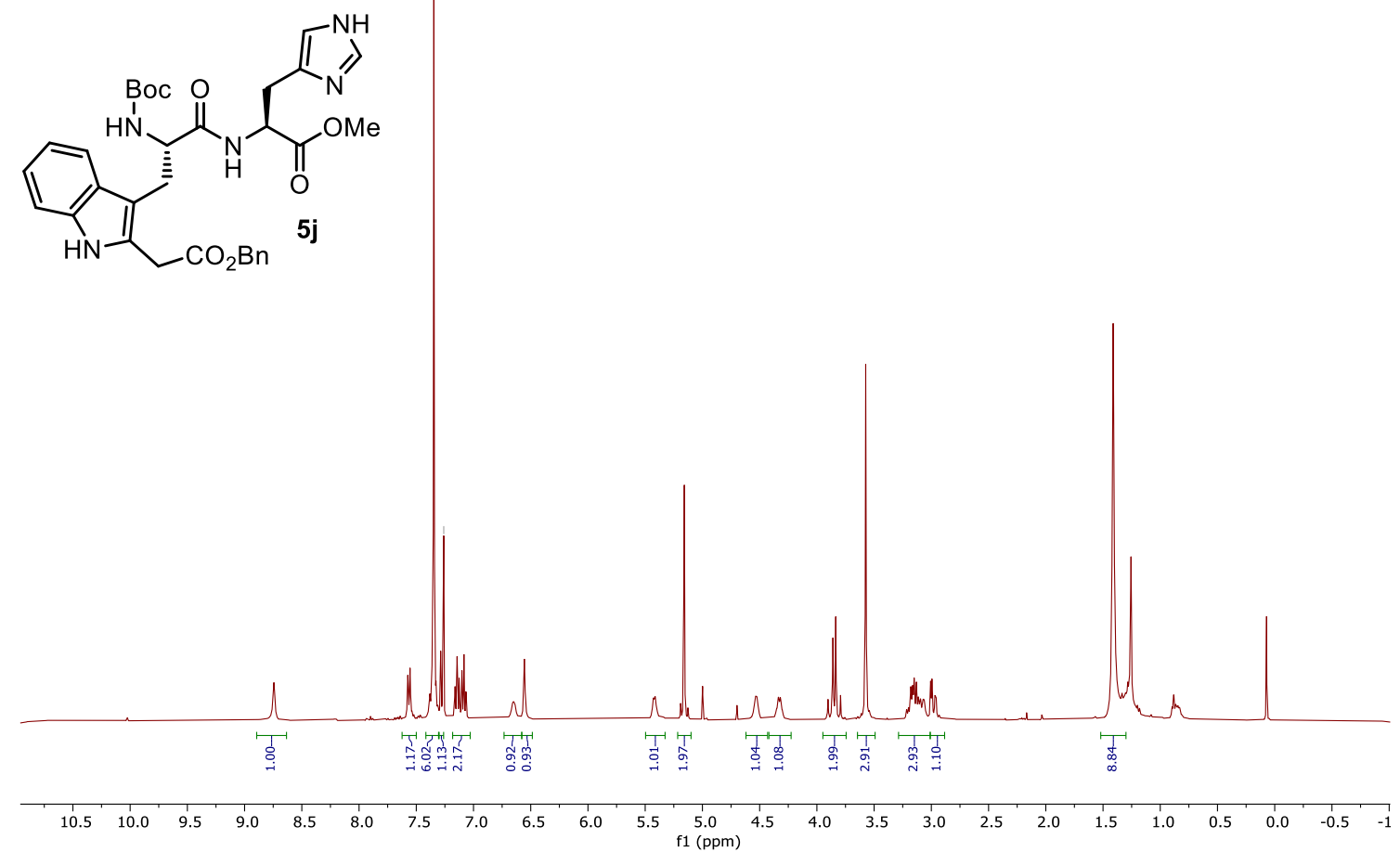

${ }^{13} \mathrm{C}$ NMR of $\mathbf{5 j}\left(101 \mathrm{MHz}, \mathrm{CDCl}_{3}\right)$

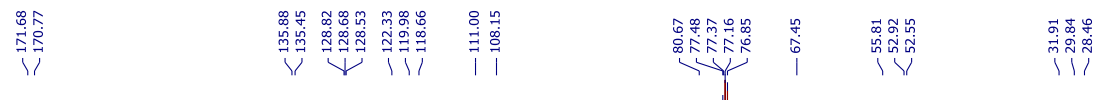

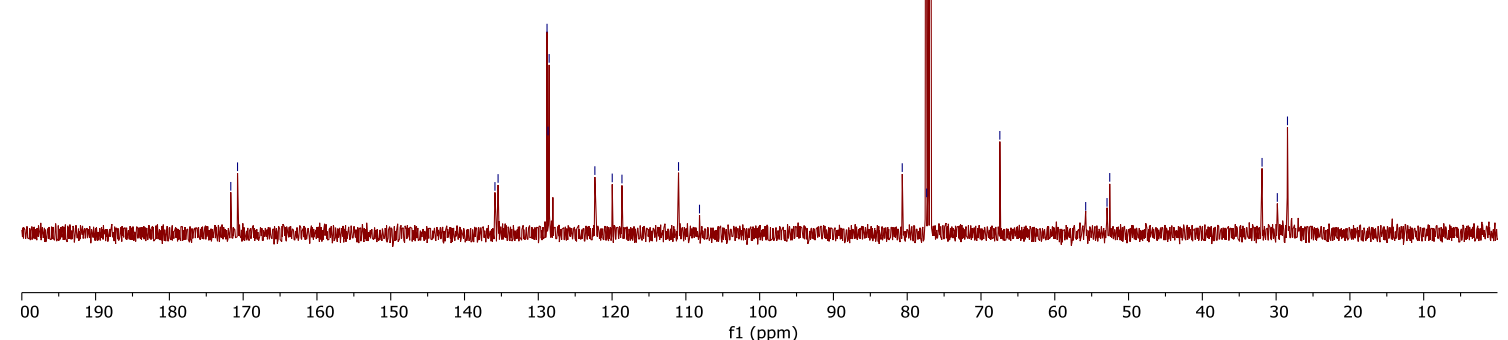


${ }^{1} \mathrm{H} \mathrm{NMR}$ of $\mathbf{5 k}\left(500 \mathrm{MHz}, \mathrm{CDCl}_{3}\right)$

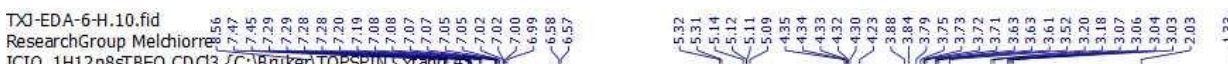

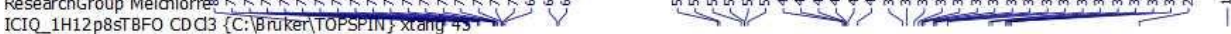

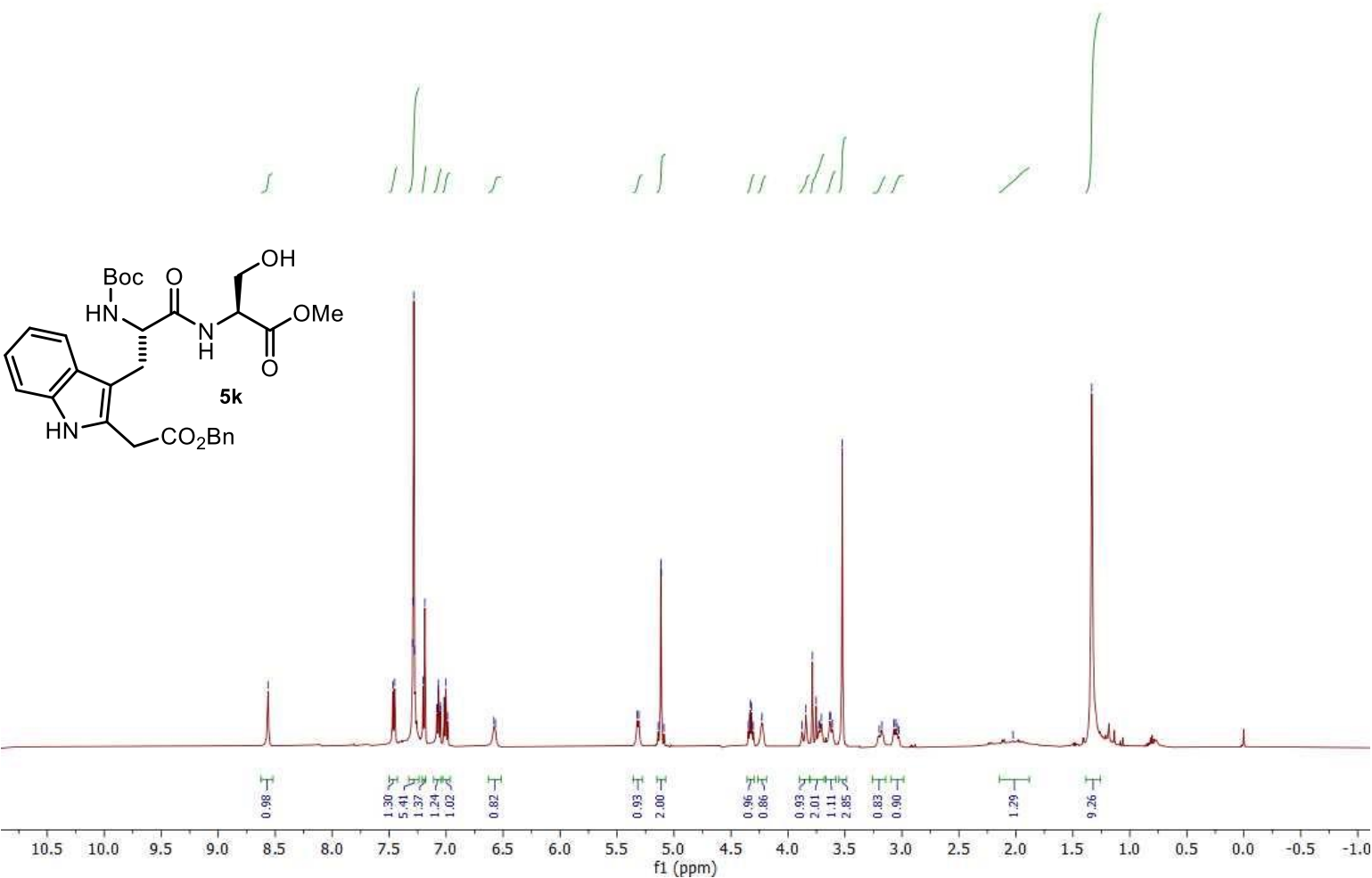

${ }^{13} \mathrm{C}$ NMR of $\mathbf{5 k}\left(126 \mathrm{MHz}, \mathrm{CDCl}_{3}\right)$

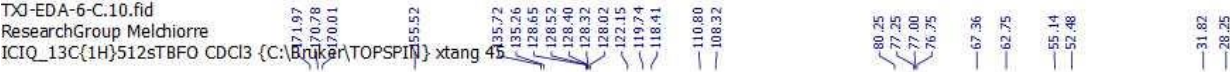

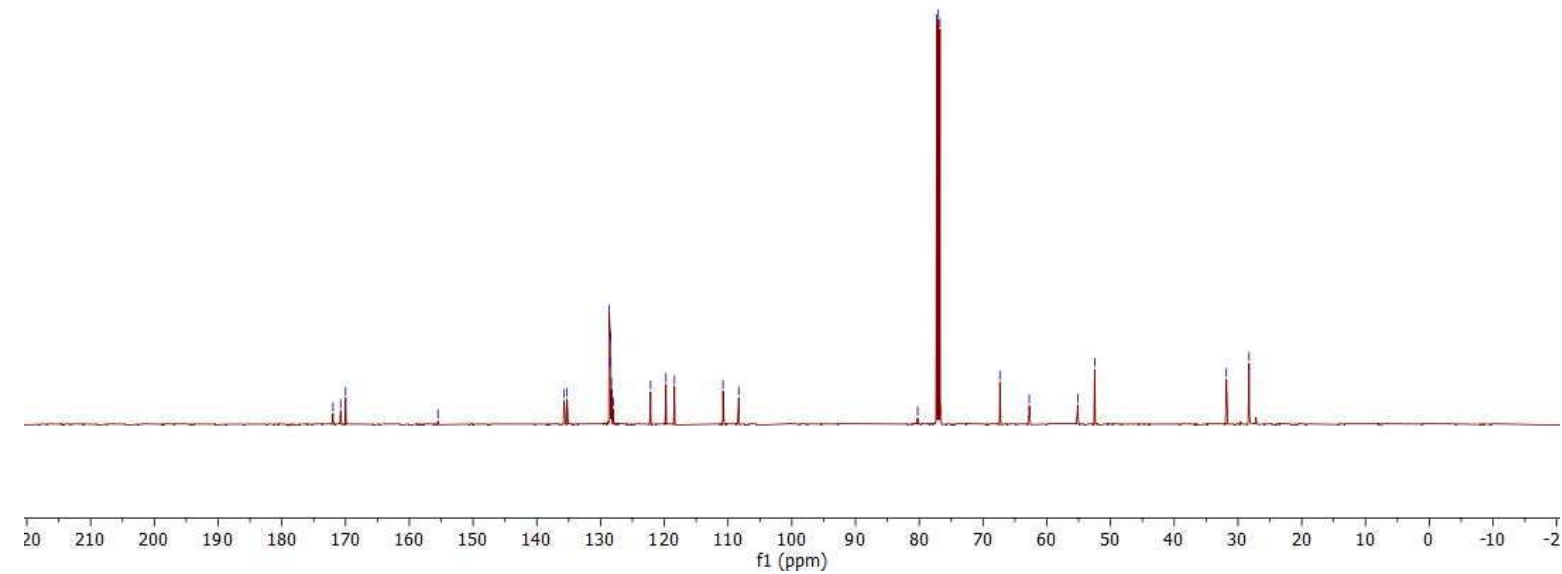


${ }^{1} \mathrm{H} \mathrm{NMR}$ of $\mathbf{5 l}\left(500 \mathrm{MHz}, \mathrm{CDCl}_{3}\right)$

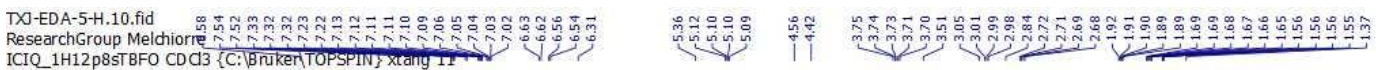

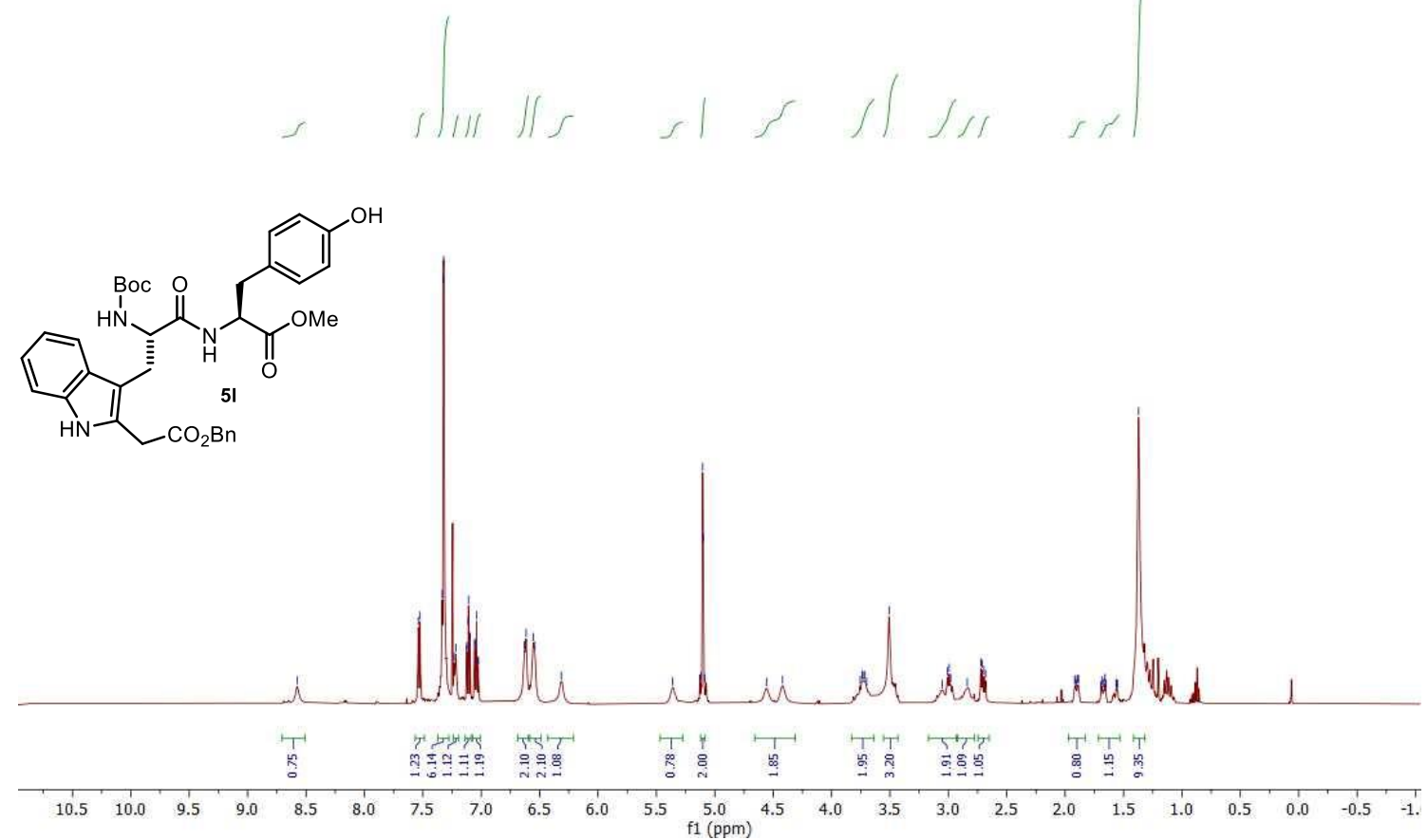

${ }^{13} \mathrm{C}$ NMR of $5 \mathbf{l}\left(126 \mathrm{MHz}, \mathrm{CDCl}_{3}\right)$

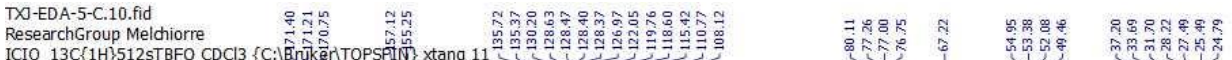

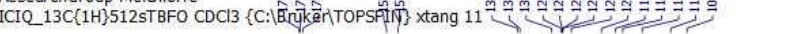

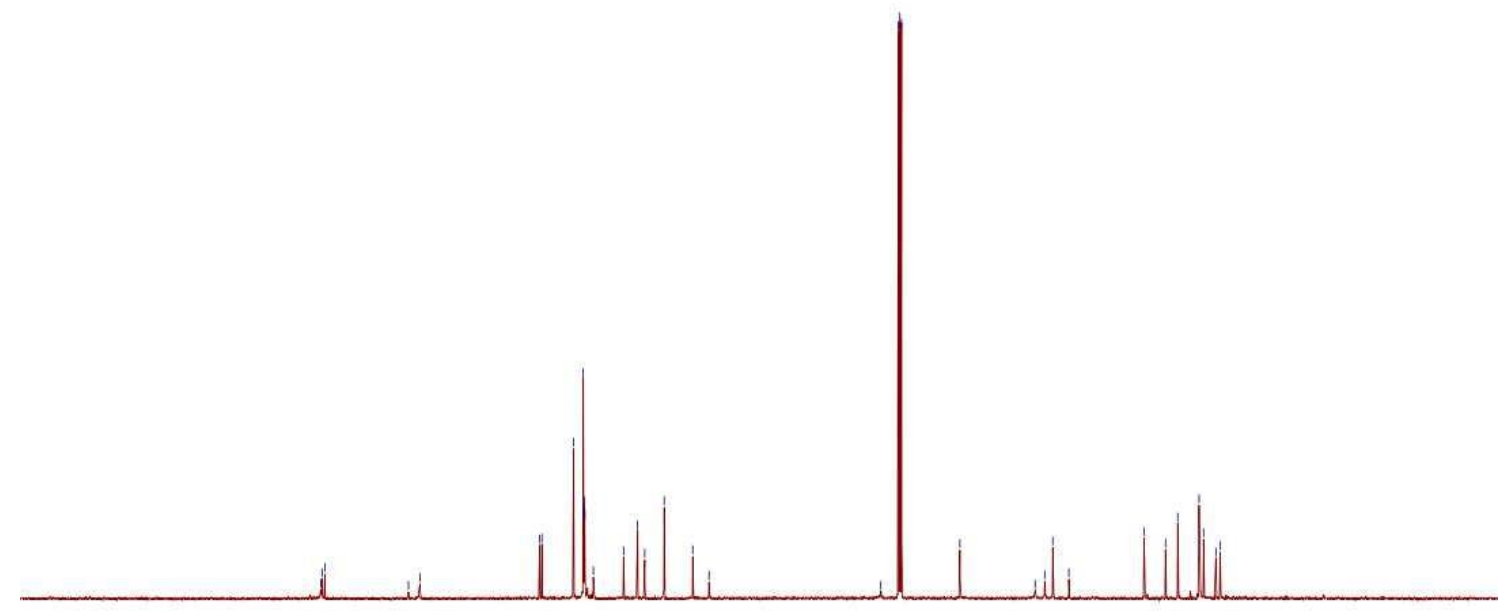

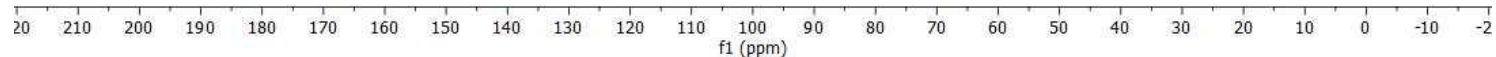


${ }^{1} \mathrm{H} \mathrm{NMR}$ of $\mathbf{5 m}\left(400 \mathrm{MHz}, \mathrm{CDCl}_{3}\right)$

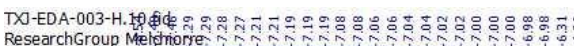
ICIQ $1 \mathrm{H} 12 \mathrm{p} 8 \mathrm{~S}$ CDClB/opt/topspin Xang 100

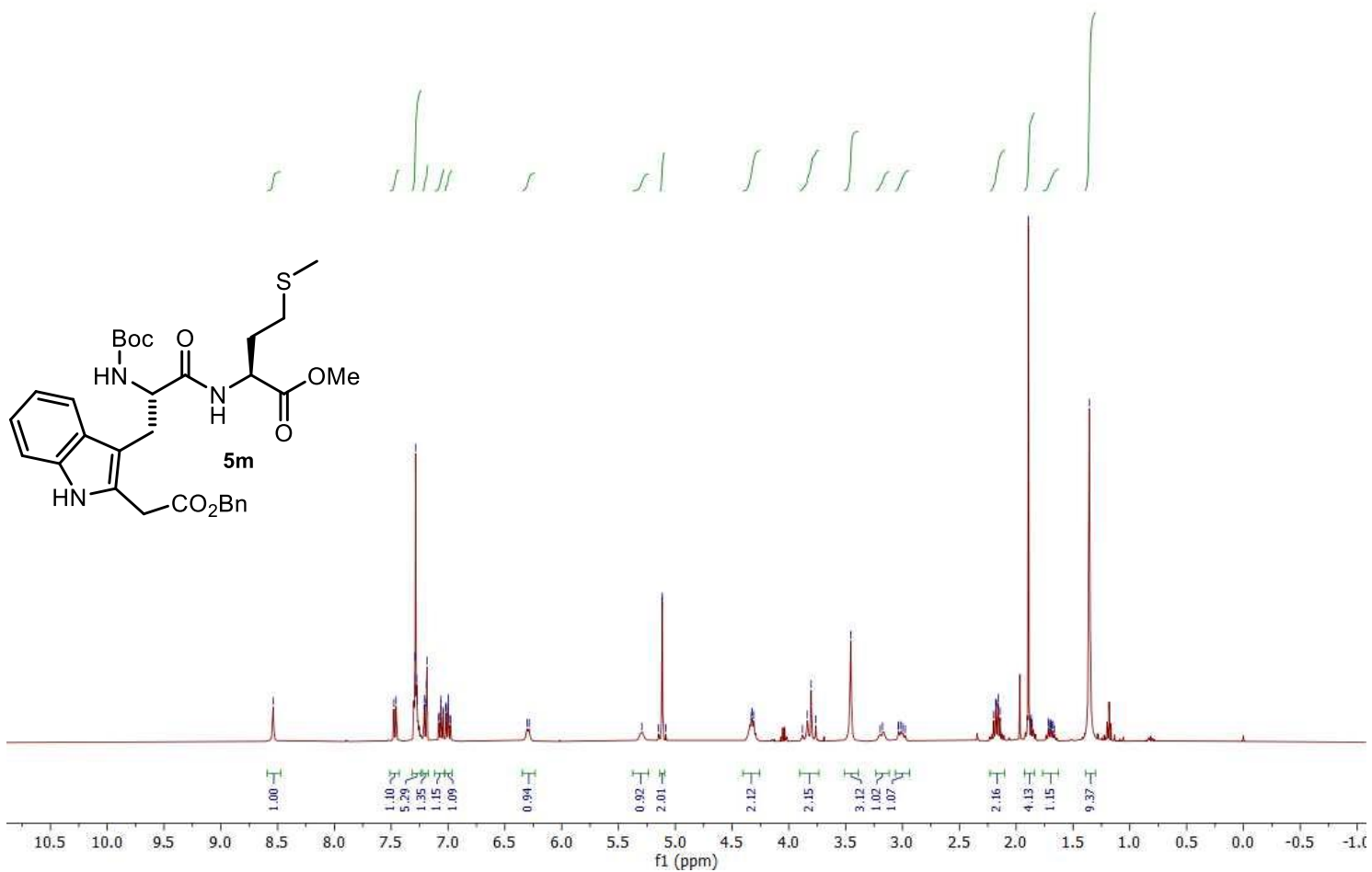

${ }^{13} \mathrm{C} \mathrm{NMR}$ of $\mathbf{5 m}\left(101 \mathrm{MHz}, \mathrm{CDCl}_{3}\right)$

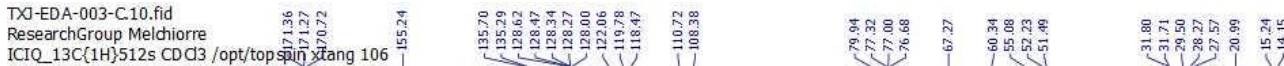

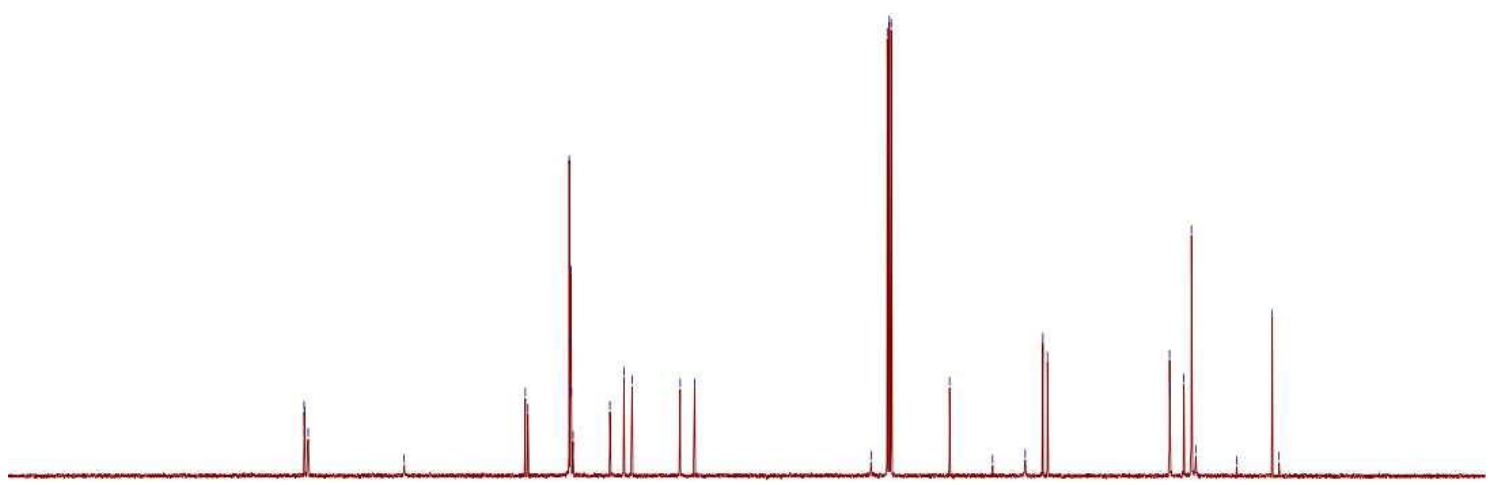

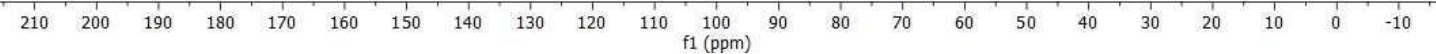


${ }^{1} \mathrm{H} \mathrm{NMR}$ of $\mathbf{5 n}\left(500 \mathrm{MHz}, \mathrm{CDCl}_{3}\right)$

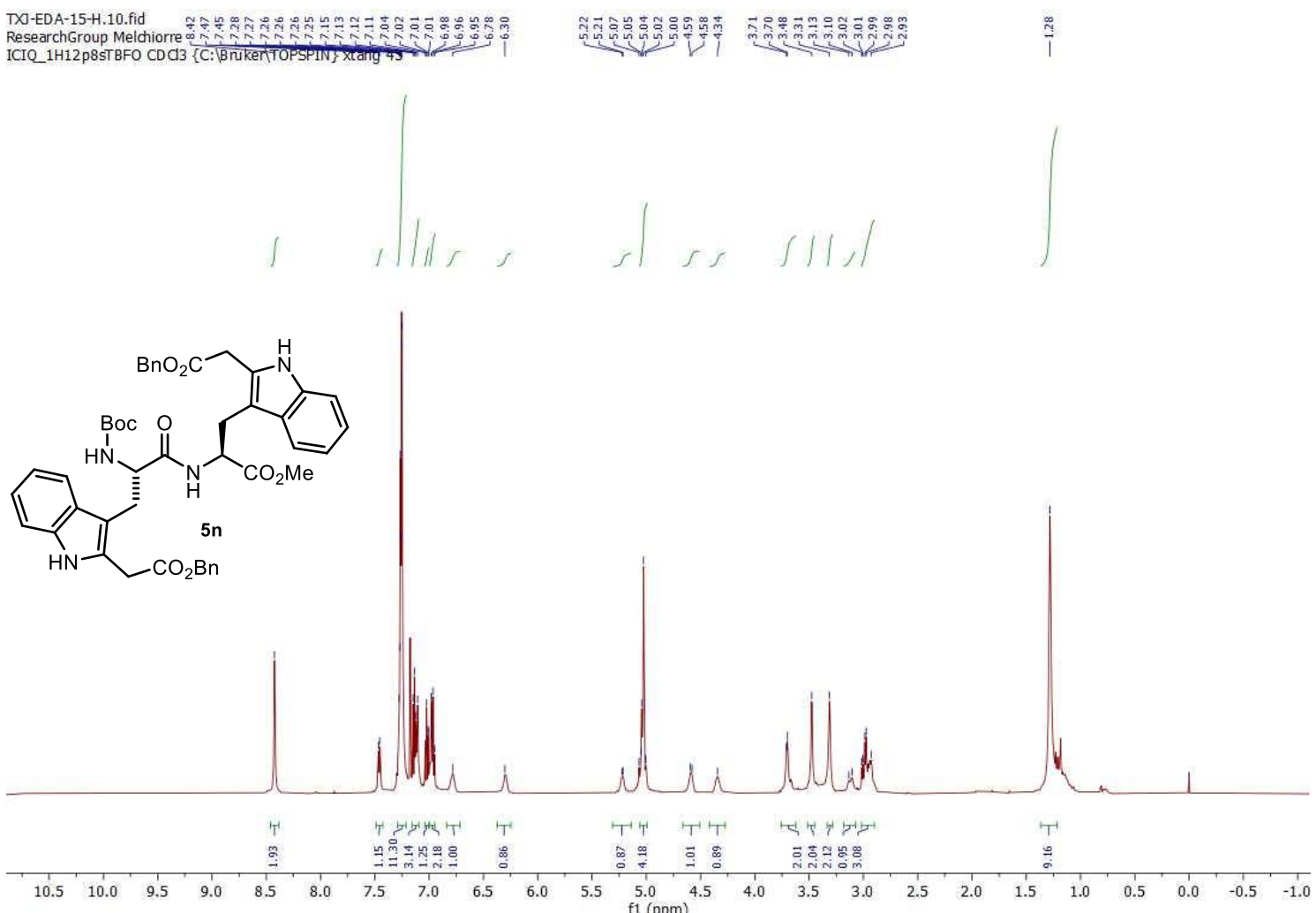

${ }^{13} \mathrm{C}$ NMR of $5 \mathbf{n}\left(126 \mathrm{MHz}, \mathrm{CDCl}_{3}\right)$

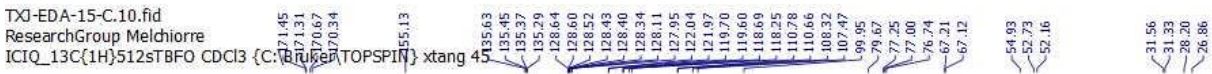

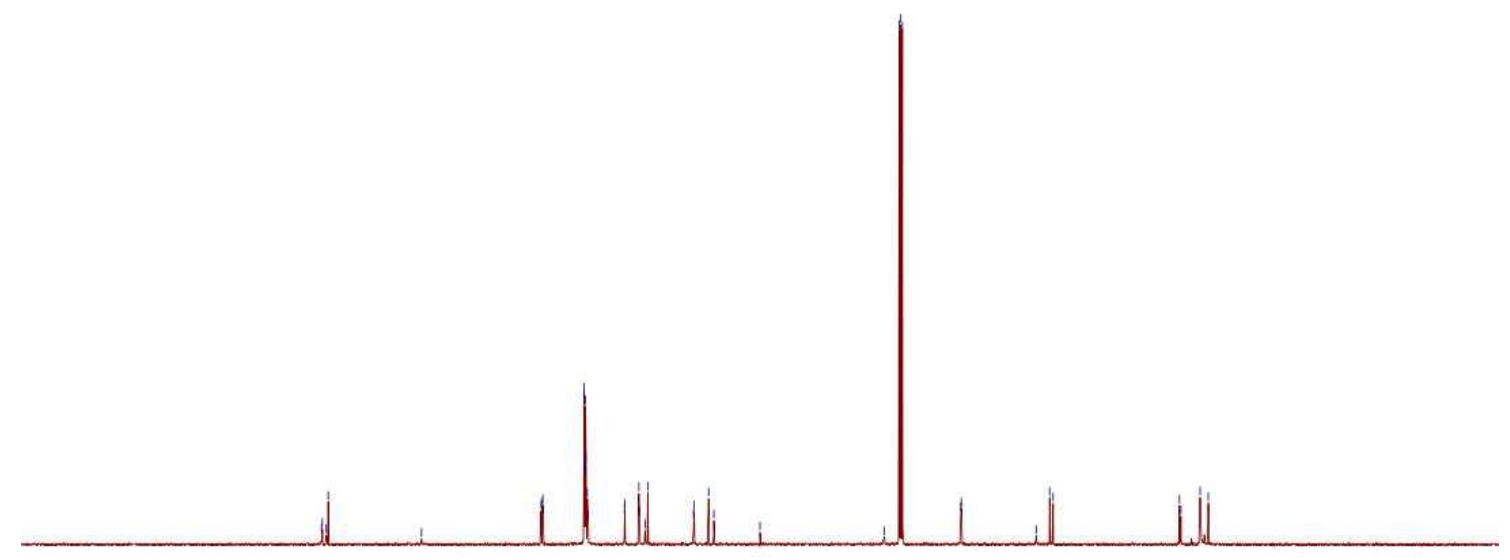

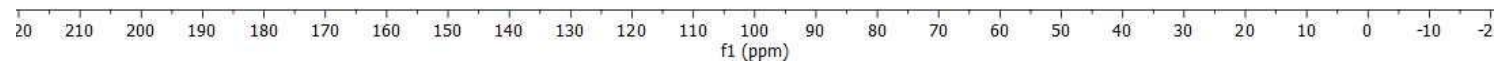


${ }^{1} \mathrm{H}$ NMR of $50\left(500 \mathrm{MHz}, \mathrm{CDCl}_{3}\right)$

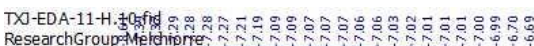

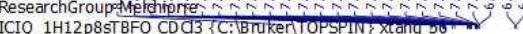

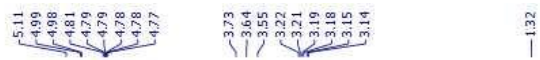

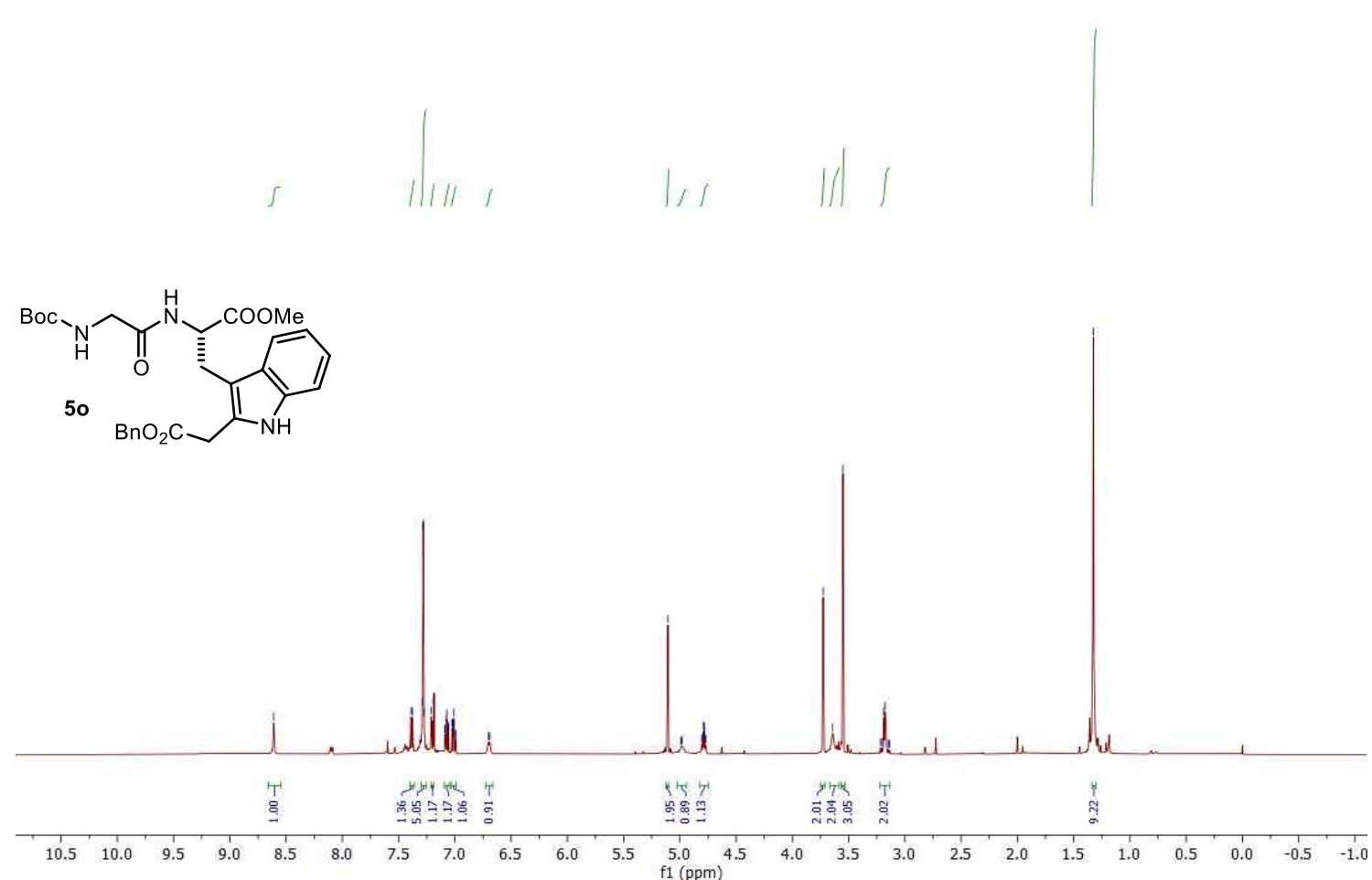

${ }^{13} \mathrm{C}$ NMR of $50\left(126 \mathrm{MHz}, \mathrm{CDCl}_{3}\right)$

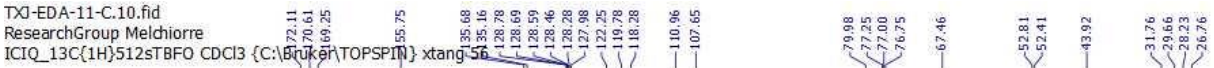

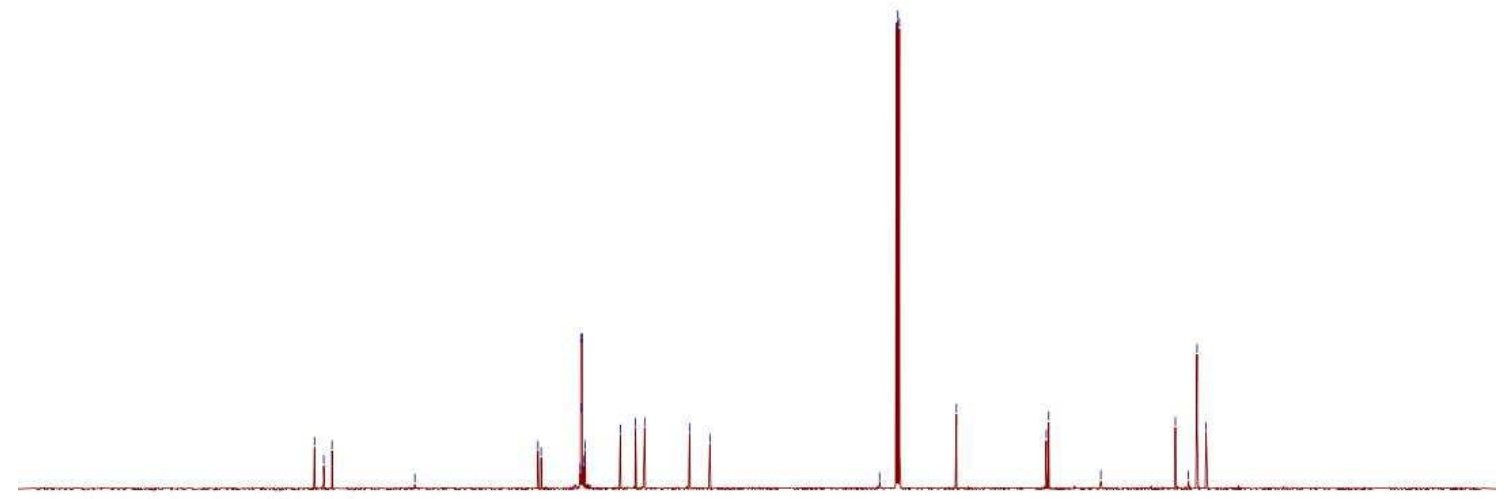

\begin{tabular}{llllllllllllllllllllllllllllllllllllll}
\hline 20 & 210 & 200 & 190 & 180 & 170 & 160 & 150 & 140 & 130 & 120 & 110 & 100 & 90 & 80 & 70 & 60 & 50 & 40 & 30 & 20 & 10 & 0 & -10 & -2
\end{tabular} 
${ }^{1} \mathrm{H}$ NMR of $\mathbf{5 p}\left(500 \mathrm{MHz}, \mathrm{CDCl}_{3}\right)$

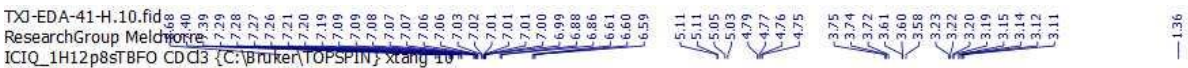

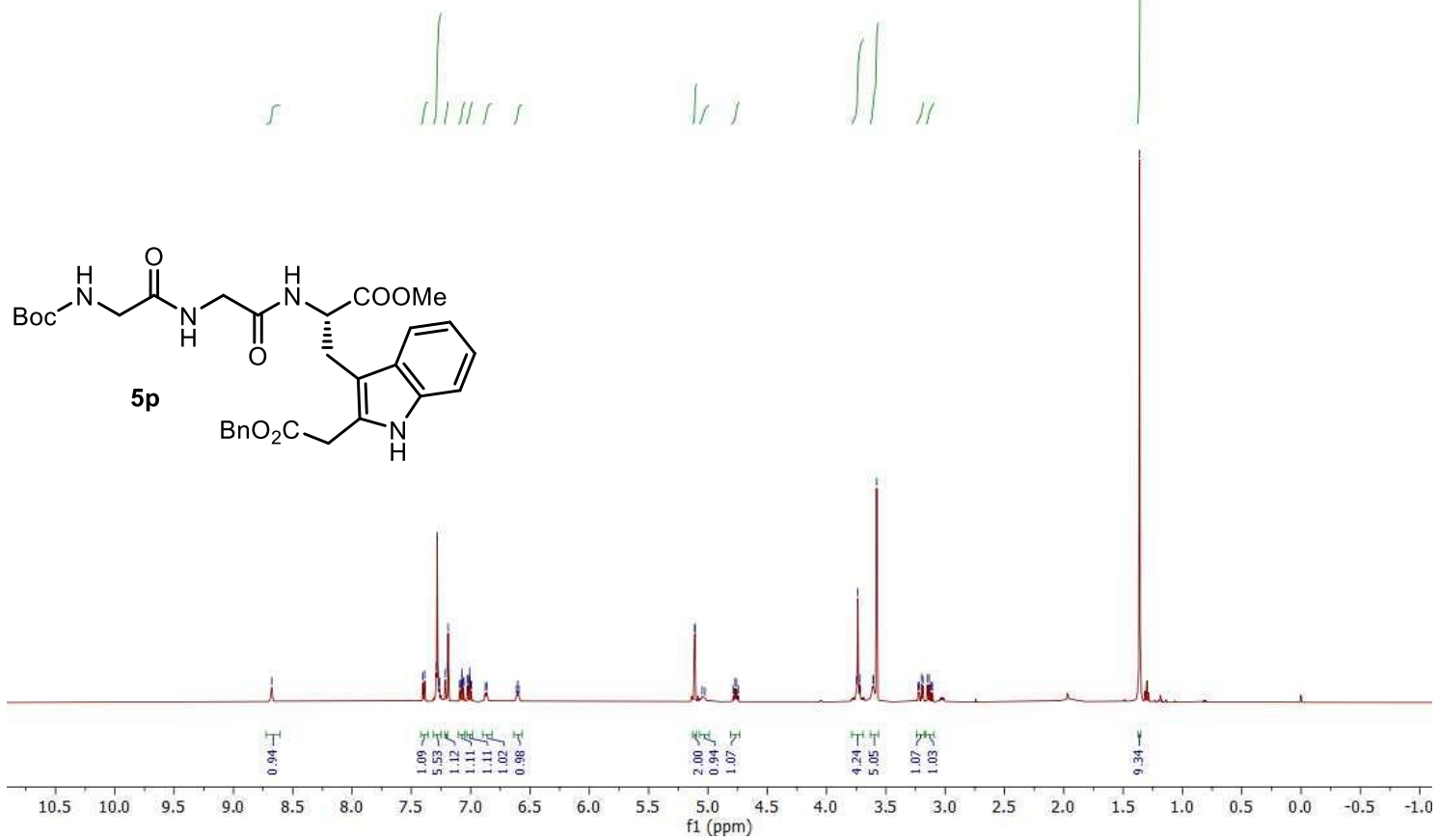

${ }^{13} \mathrm{C}$ NMR of $\mathbf{5 p}\left(126 \mathrm{MHz}, \mathrm{CDCl}_{3}\right)$

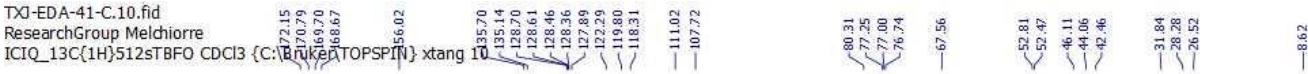

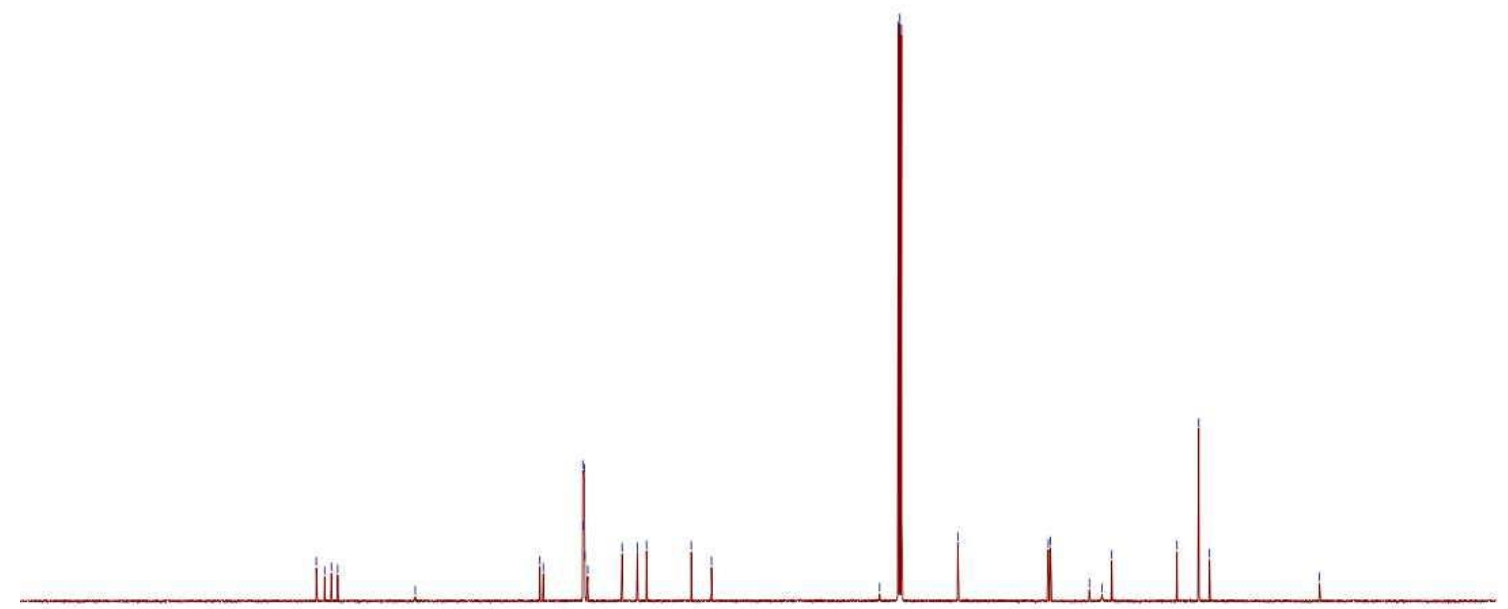

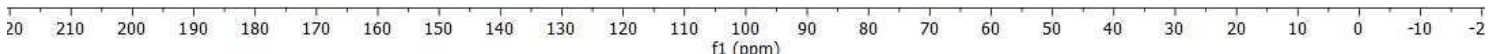


${ }^{1} \mathrm{H} \mathrm{NMR}$ of $\mathbf{5 q}\left(500 \mathrm{MHz}, \mathrm{CDCl}_{3}\right)$

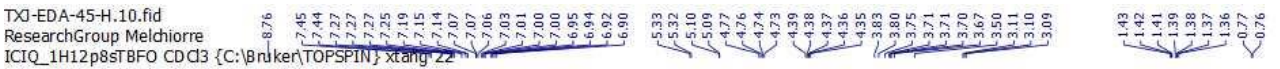

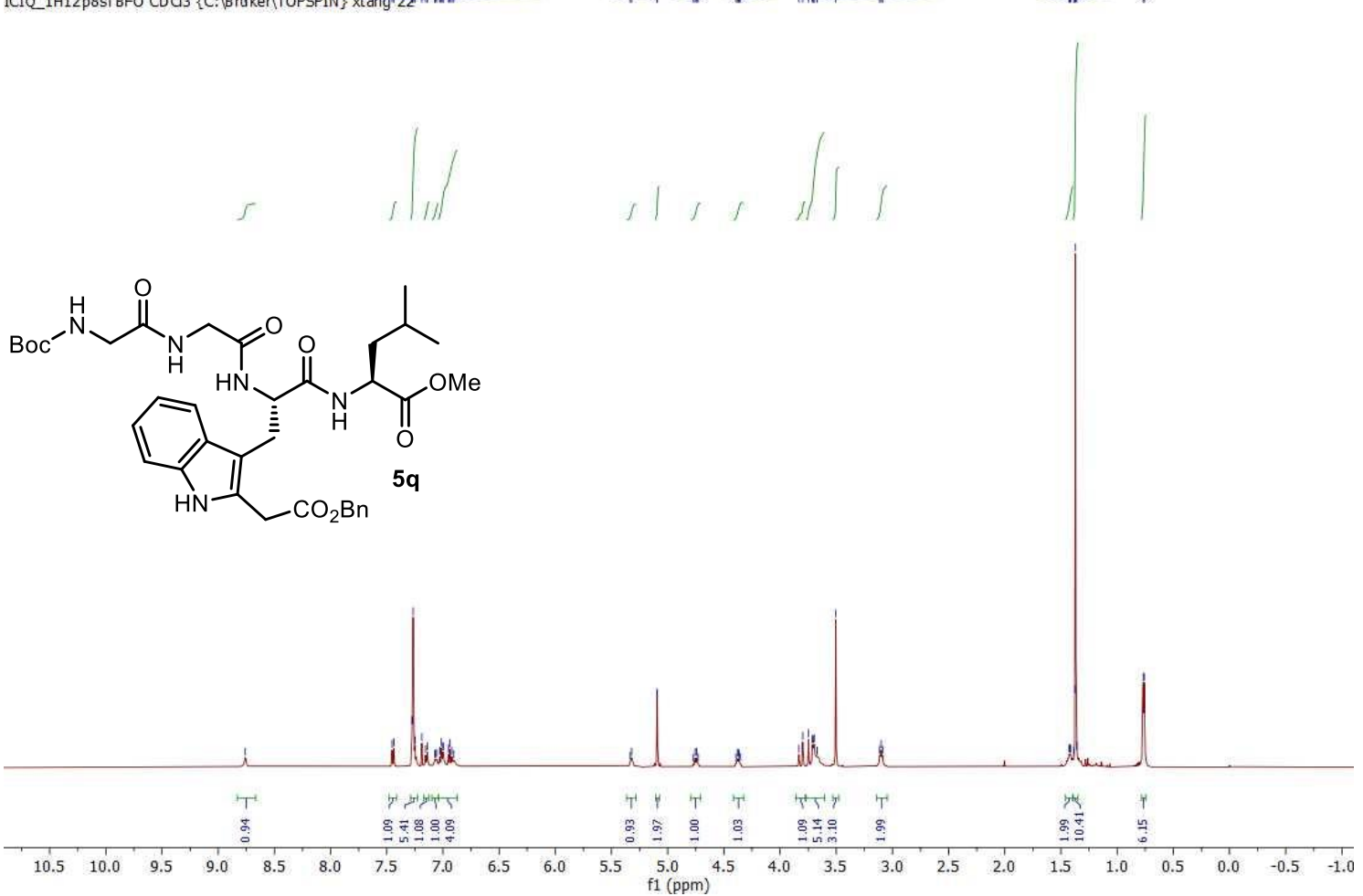

${ }^{13} \mathrm{C}$ NMR of $\mathbf{5 q}\left(126 \mathrm{MHz}, \mathrm{CDCl}_{3}\right)$

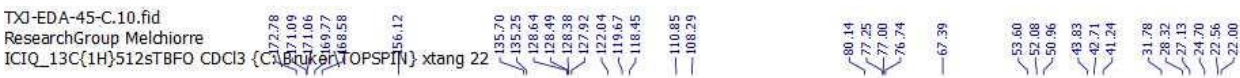

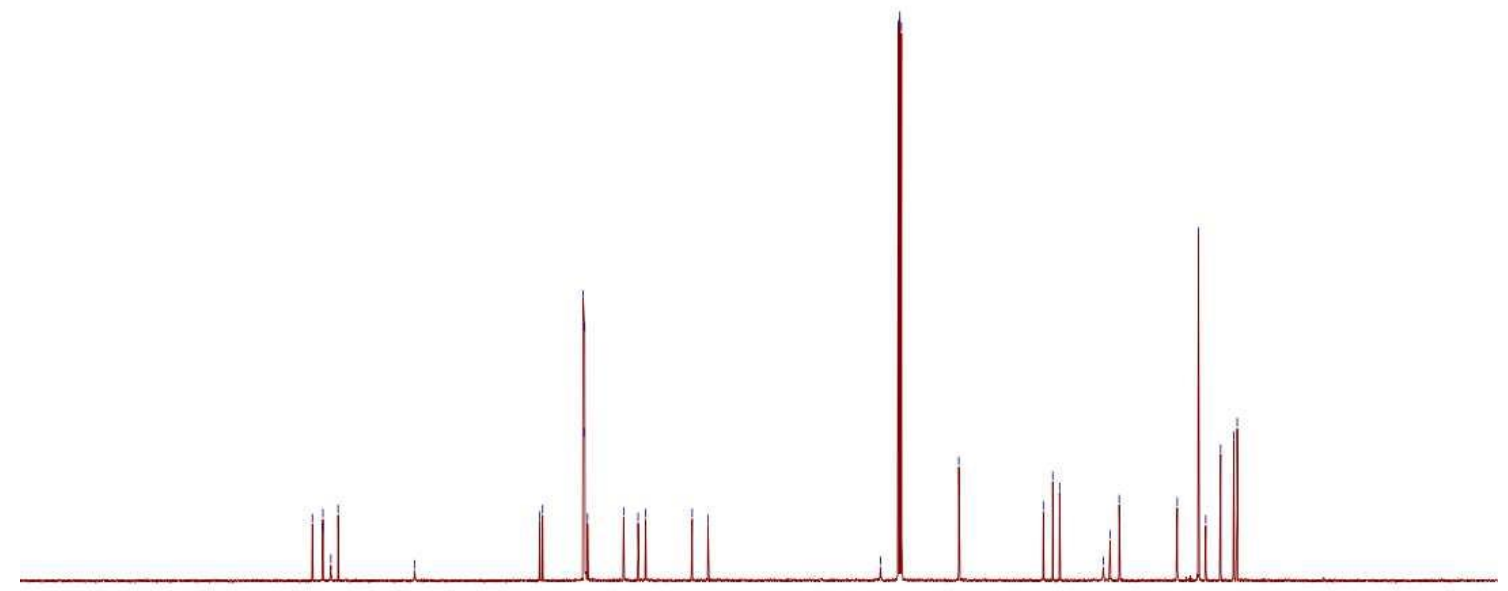

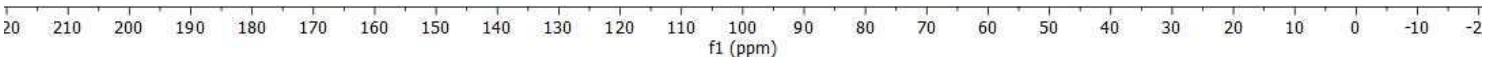


${ }^{1} \mathrm{H}$ NMR of $6 \mathbf{a}\left(400 \mathrm{MHz}, \mathrm{CDCl}_{3}\right)$

BPL168-pure.20.fid

ICIQ 1 H12pss CDCl3 /opt/topspin blardche 100 in

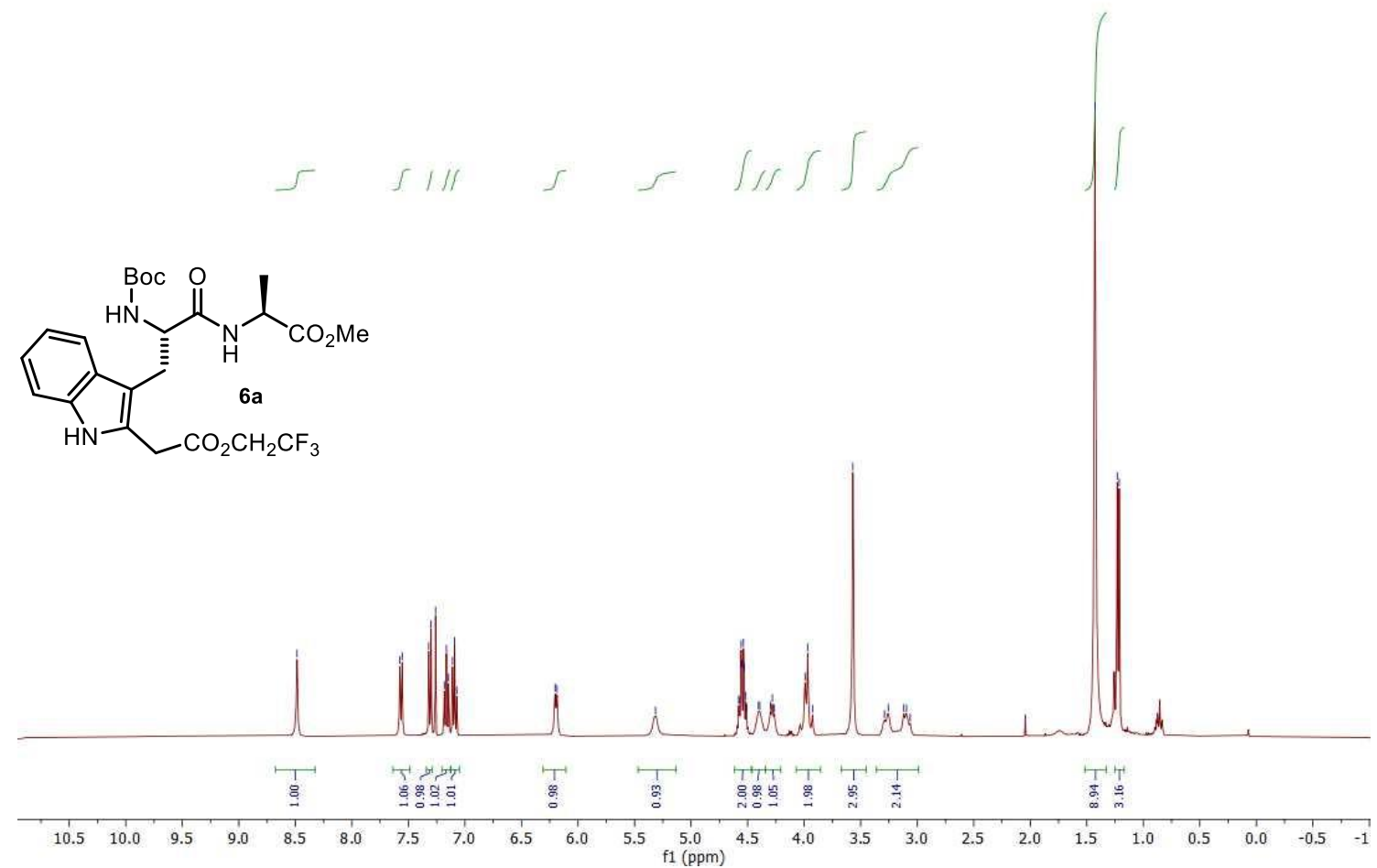

${ }^{13} \mathrm{C}$ NMR of $6 \mathbf{a}\left(101 \mathrm{MHz}, \mathrm{CDCl}_{3}\right)$

BPL168-C13.10.fid

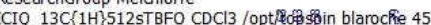

il

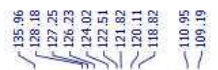

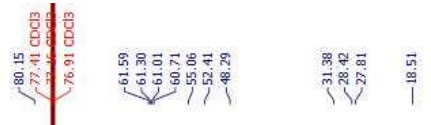

\begin{tabular}{lllllllllllllllllllllllll}
\hline 20 & 210 & 200 & 190 & 180 & 170 & 160 & 150 & 140 & 130 & 120 & 110 & 100 & 90 & 10 & 70 & 60 & 50 & 40 & 30 & 20 & 10 & 0 & -10 & -2
\end{tabular} 
${ }^{1} \mathrm{H}$ NMR of $6 \mathbf{b}\left(400 \mathrm{MHz}, \mathrm{CDCl}_{3}\right)$

BPL179-pure.10.fid

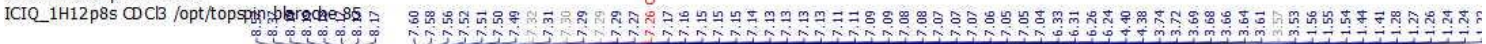

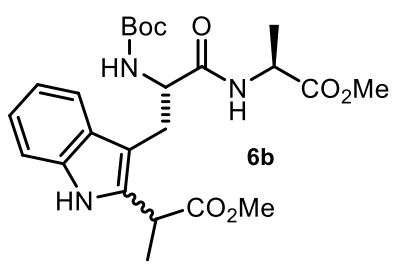

il ill H il

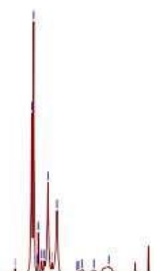

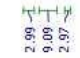

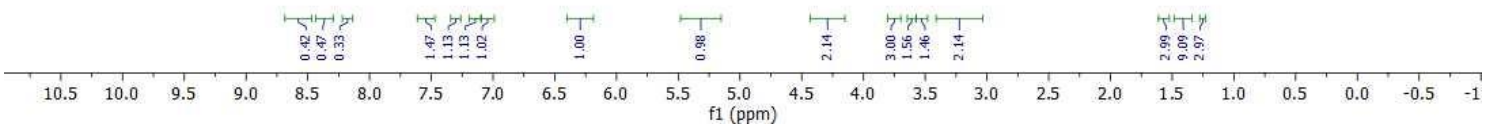

${ }^{13} \mathrm{C}$ NMR of $\mathbf{6 b}\left(101 \mathrm{MHz}, \mathrm{CDCl}_{3}\right)$

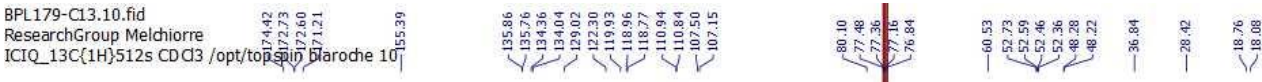

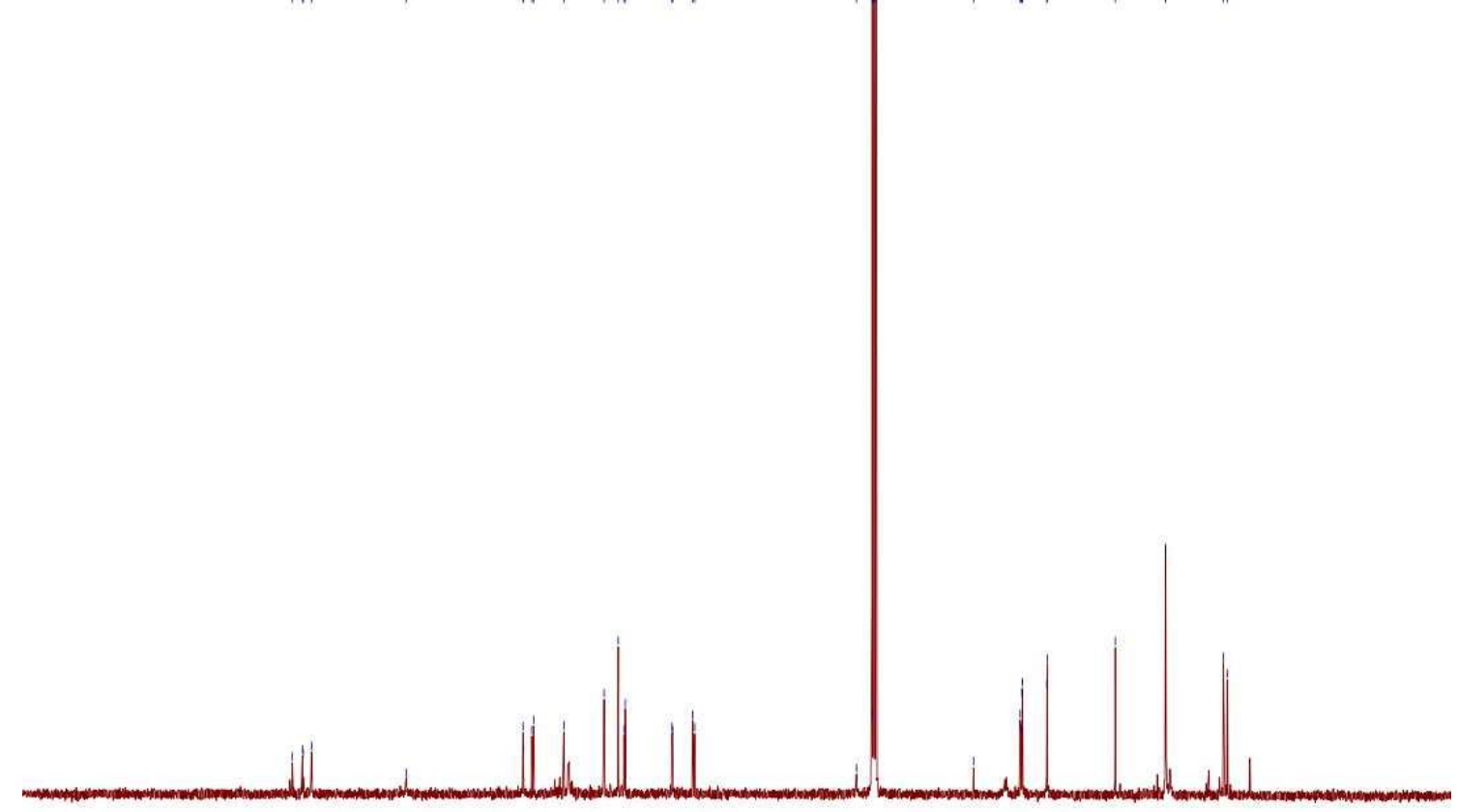

\begin{tabular}{lllllllllllllllllllllllll}
\hline & 10 & 200 & 190 & 180 & 170 & 160 & 150 & 140 & 130 & 120 & 110 & 100 & 90 & 80 & 70 & 60 & 50 & 40 & 30 & 20 & 10 & 0 & -10
\end{tabular} 
${ }^{1} \mathrm{H}$ NMR of $\mathbf{6 c}\left(400 \mathrm{MHz}, \mathrm{CDCl}_{3}\right)$

BPL178-pure.10.fid

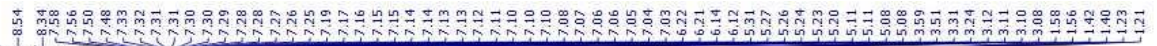
ICIQ $1 \mathrm{H} 12$ p8s CDCl3 /opt/topspin blaroche 103

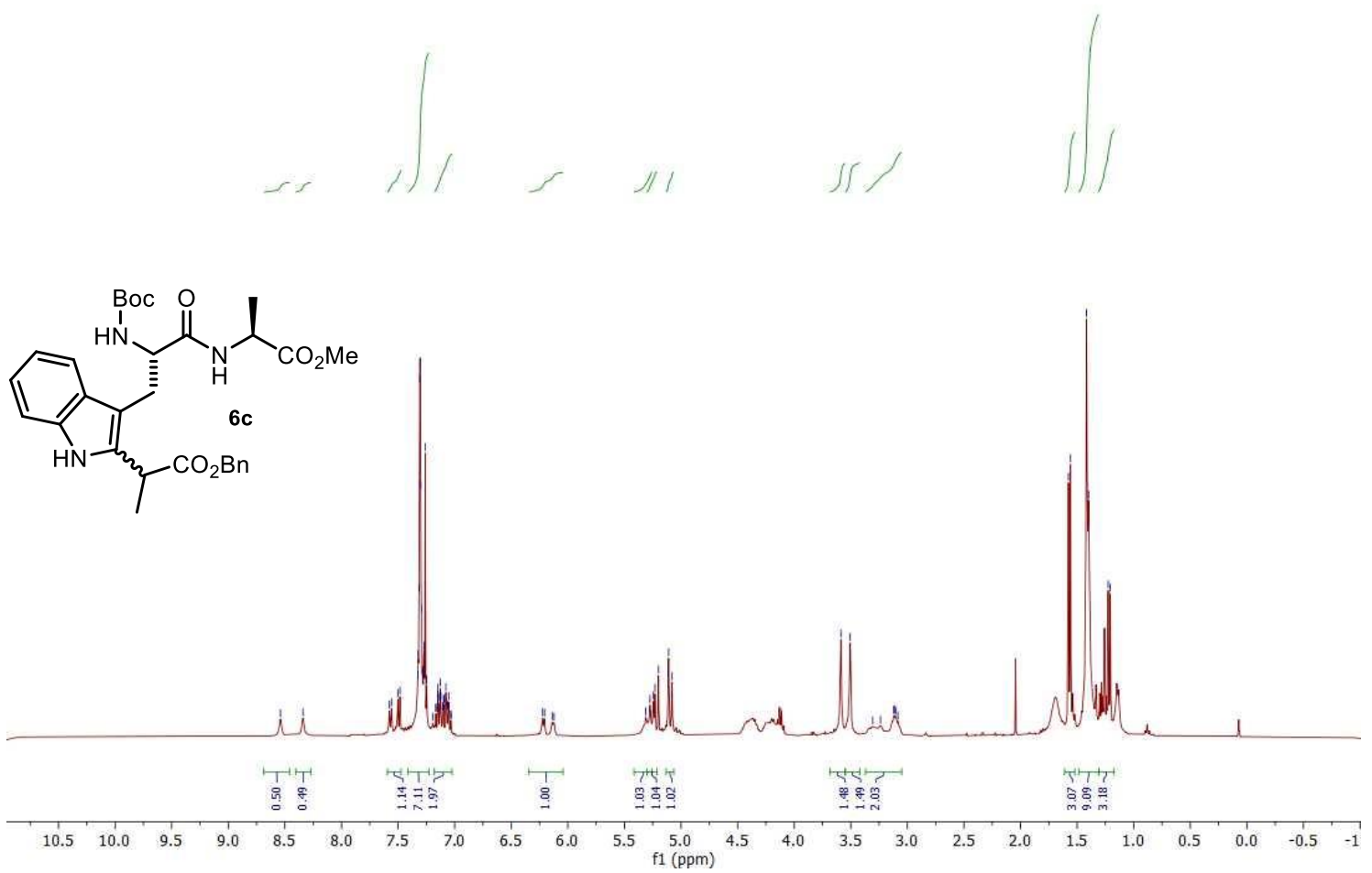

${ }^{13} \mathrm{C}$ NMR of $\mathbf{6 c}\left(126 \mathrm{MHz}, \mathrm{CDCl}_{3}\right)$

BPL178-C13.10.fid

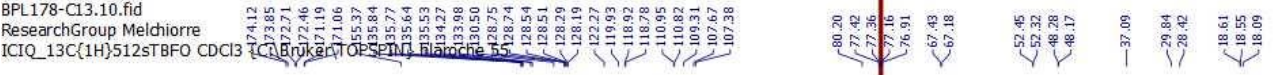

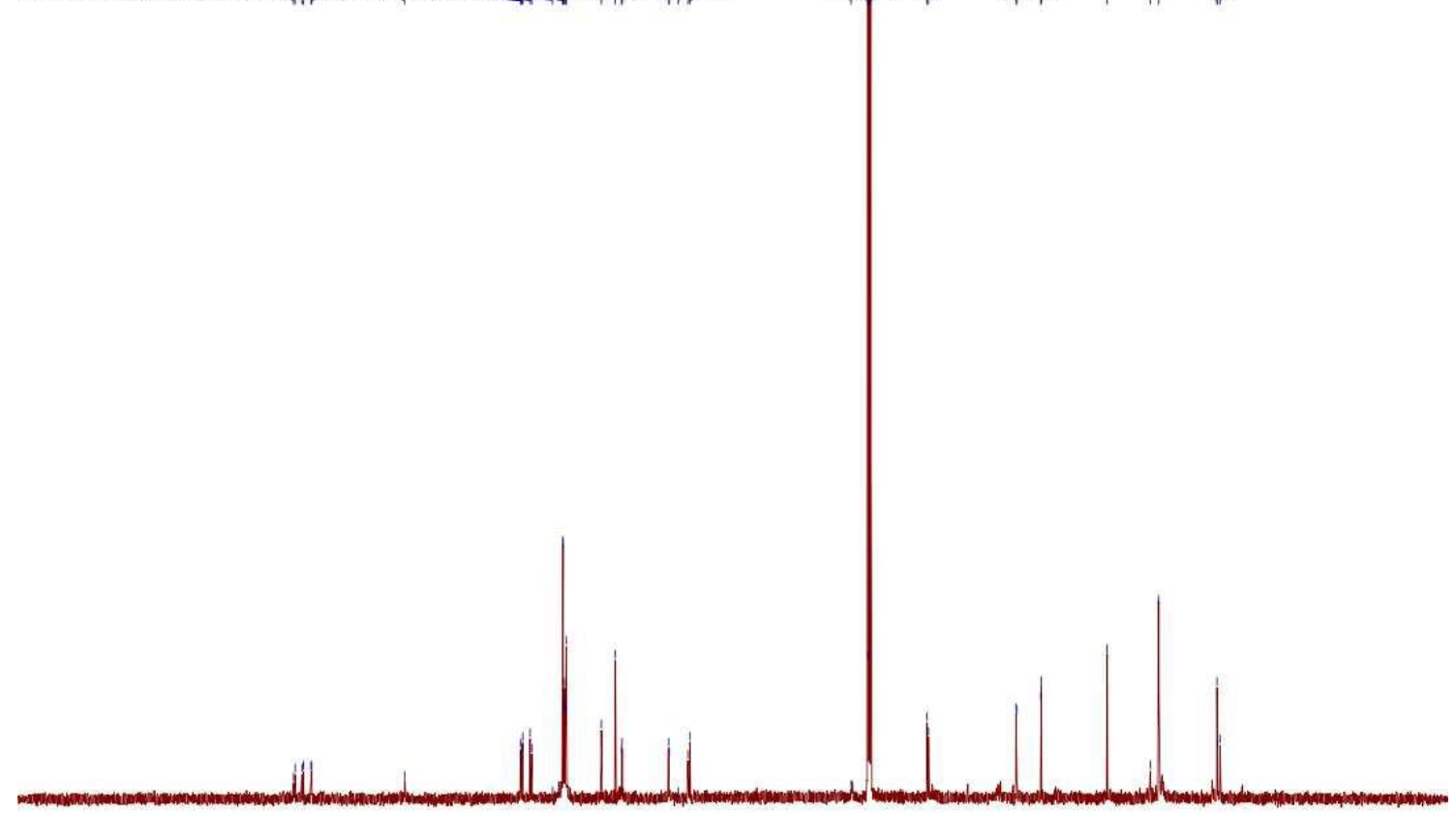

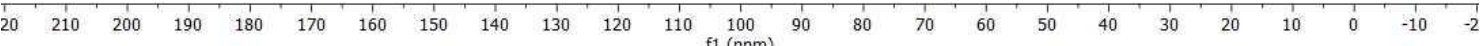


${ }^{1} \mathrm{H}$ NMR of $\mathbf{6 d}\left(400 \mathrm{MHz}, \mathrm{CDCl}_{3}\right)$

BPL180-pure.30.fid
ResearchGroup Meldhiorre

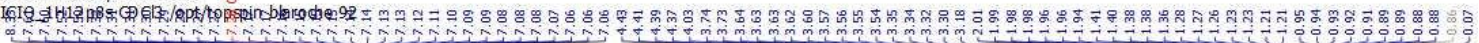

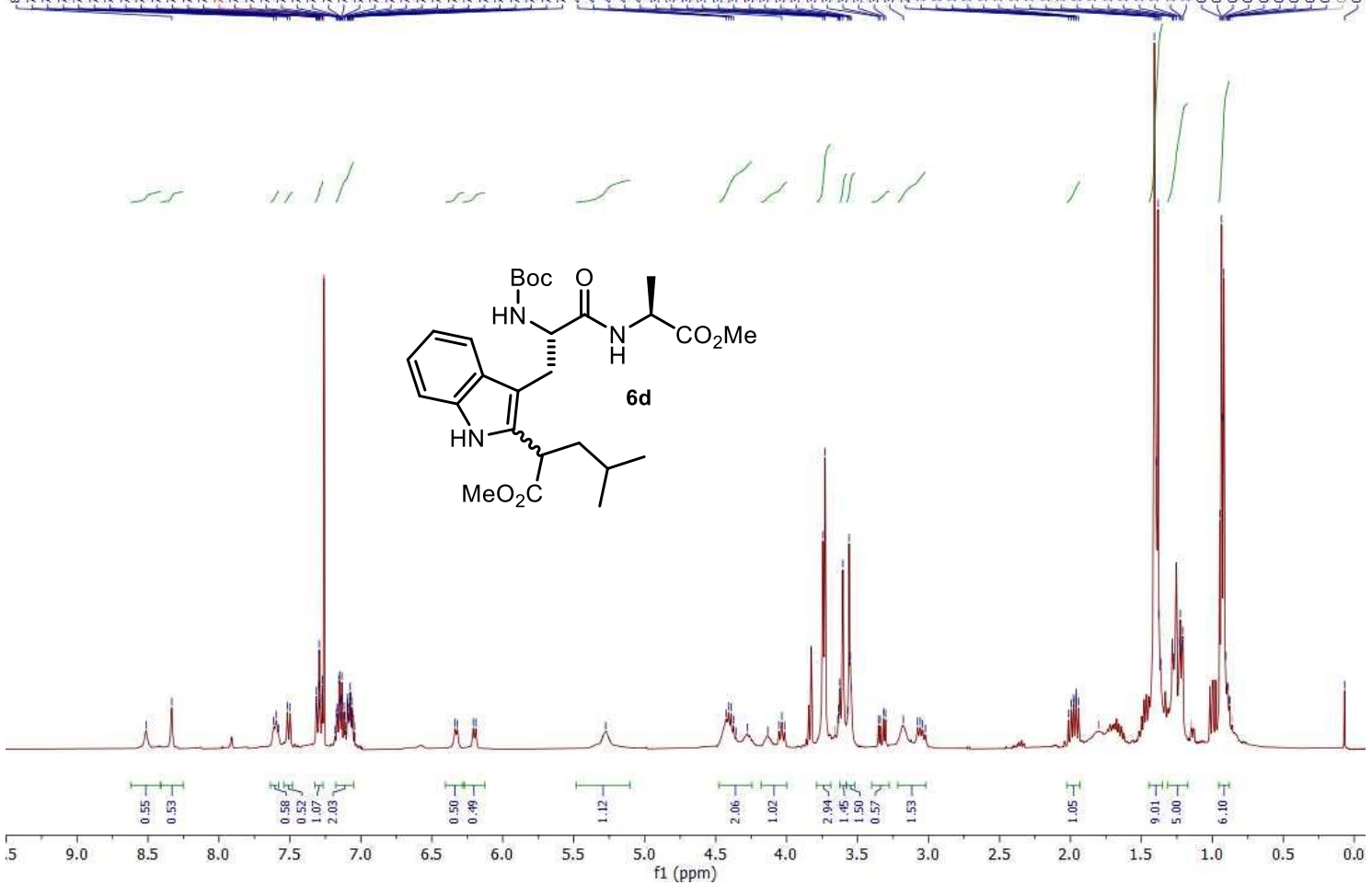

${ }^{13} \mathrm{C}$ NMR of $\mathbf{6 d}\left(101 \mathrm{MHz}, \mathrm{CDCl}_{3}\right)$

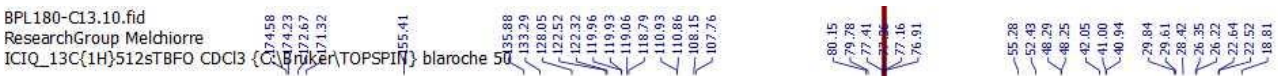

年

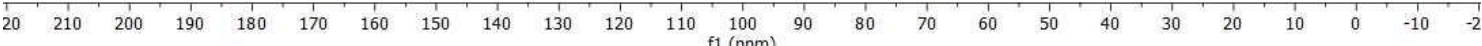


${ }^{1} \mathrm{H}$ NMR of $6 \mathbf{e}\left(400 \mathrm{MHz}, \mathrm{CDCl}_{3}\right)$

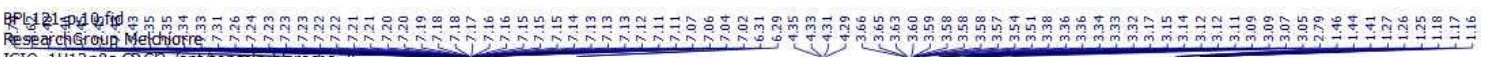

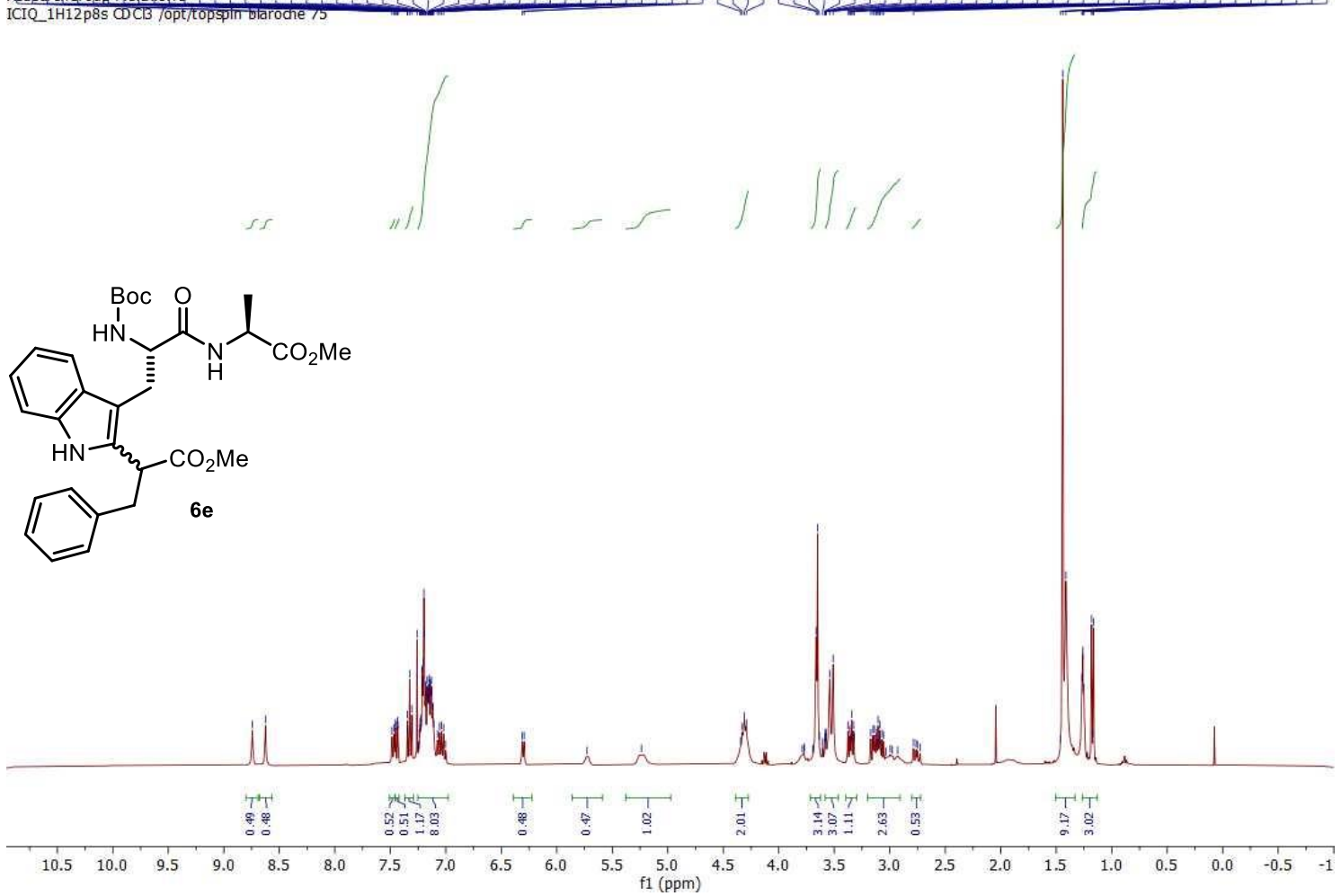

${ }^{13} \mathrm{C}$ NMR of $6 \mathbf{e}\left(126 \mathrm{MHz}, \mathrm{CDCl}_{3}\right)$

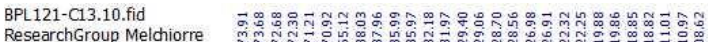

ResearchGroup Melchiorre 1 m

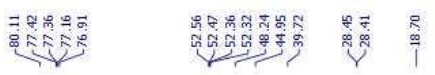

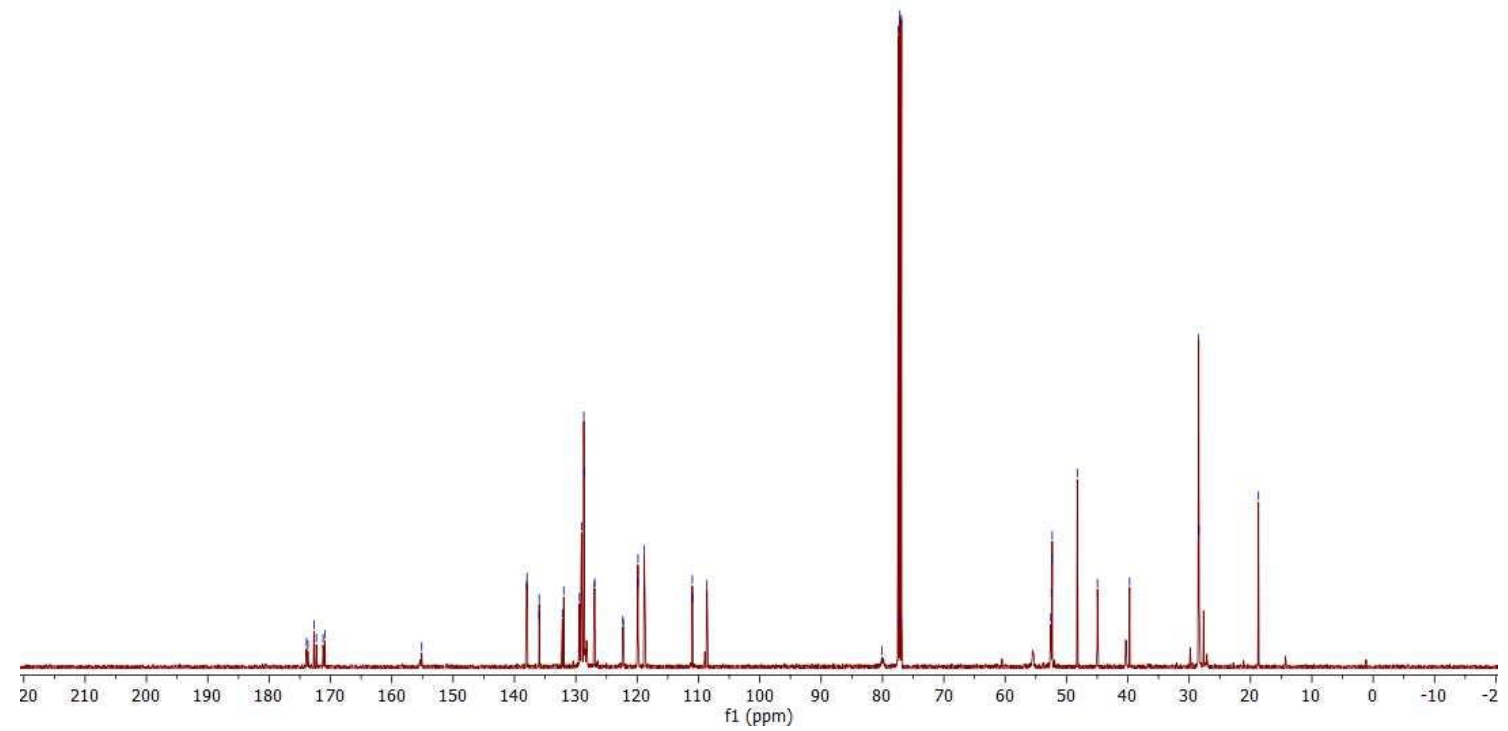


${ }^{1} \mathrm{H} \mathrm{NMR}$ of $\boldsymbol{6} \mathbf{f}\left(300 \mathrm{MHz}, \mathrm{CDCl}_{3}\right)$

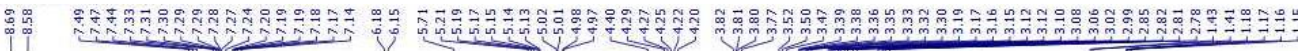

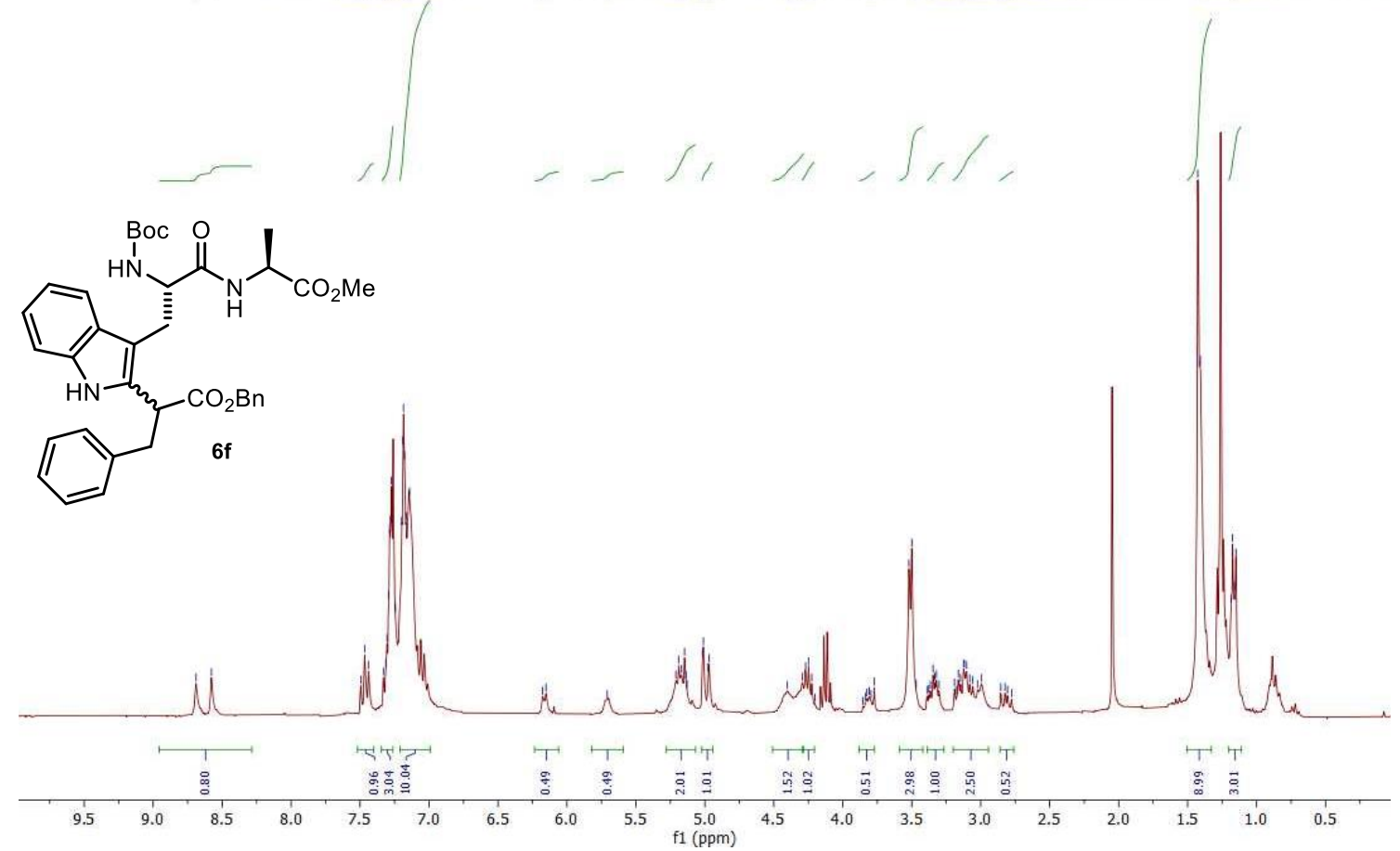

${ }^{13} \mathrm{C}$ NMR of $6 \mathbf{f}\left(126 \mathrm{MHz}, \mathrm{CDCl}_{3}\right)$

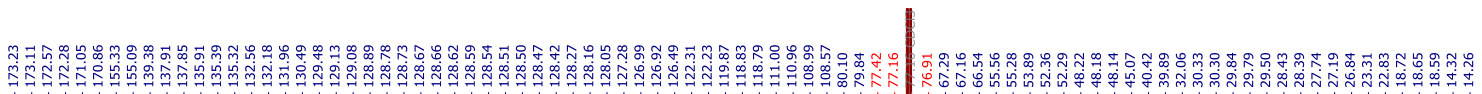

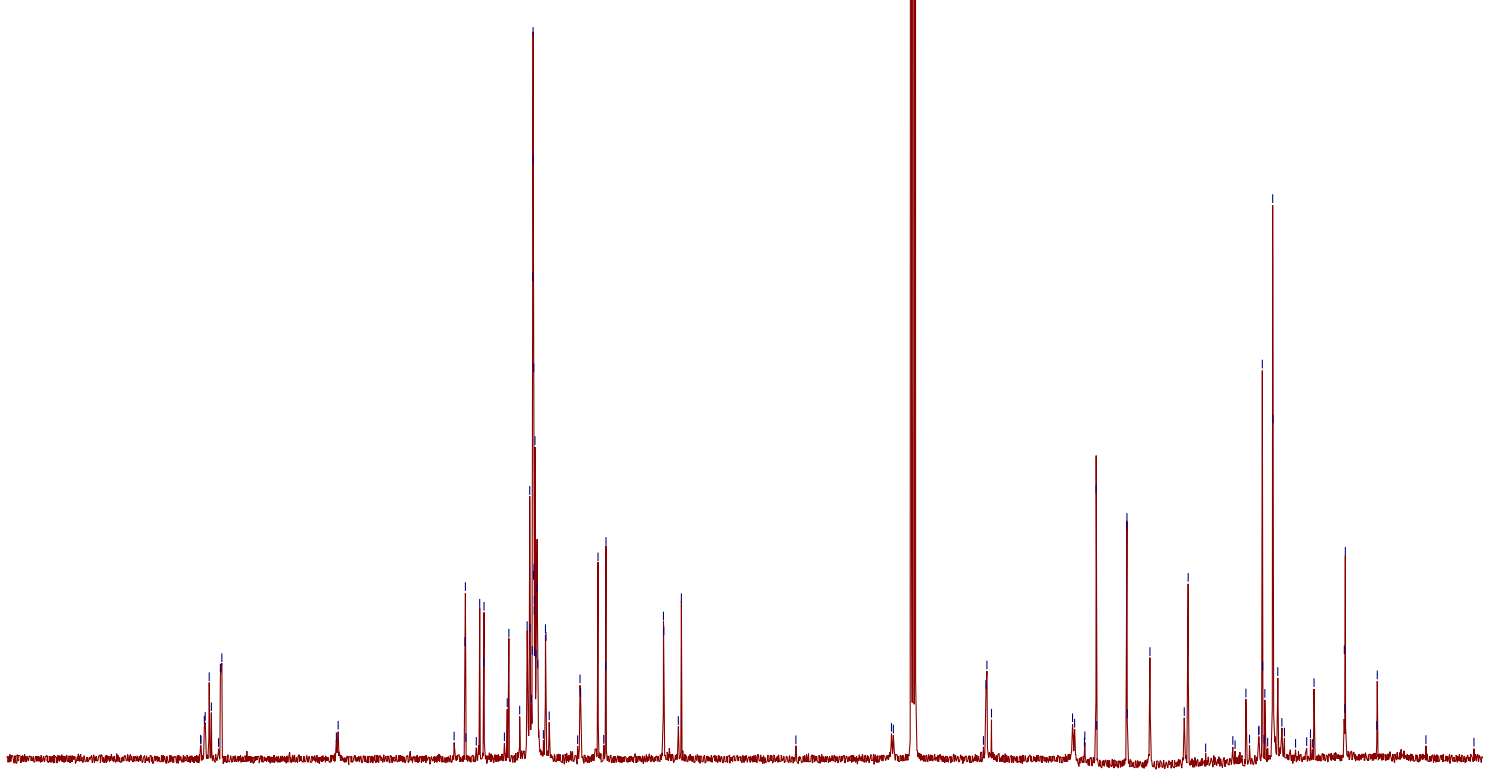

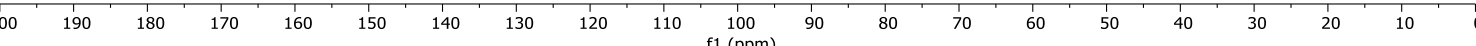


${ }^{1} \mathrm{H} \mathrm{NMR}$ of $\mathbf{6 g}\left(300 \mathrm{MHz}, \mathrm{CDCl}_{3}\right)$

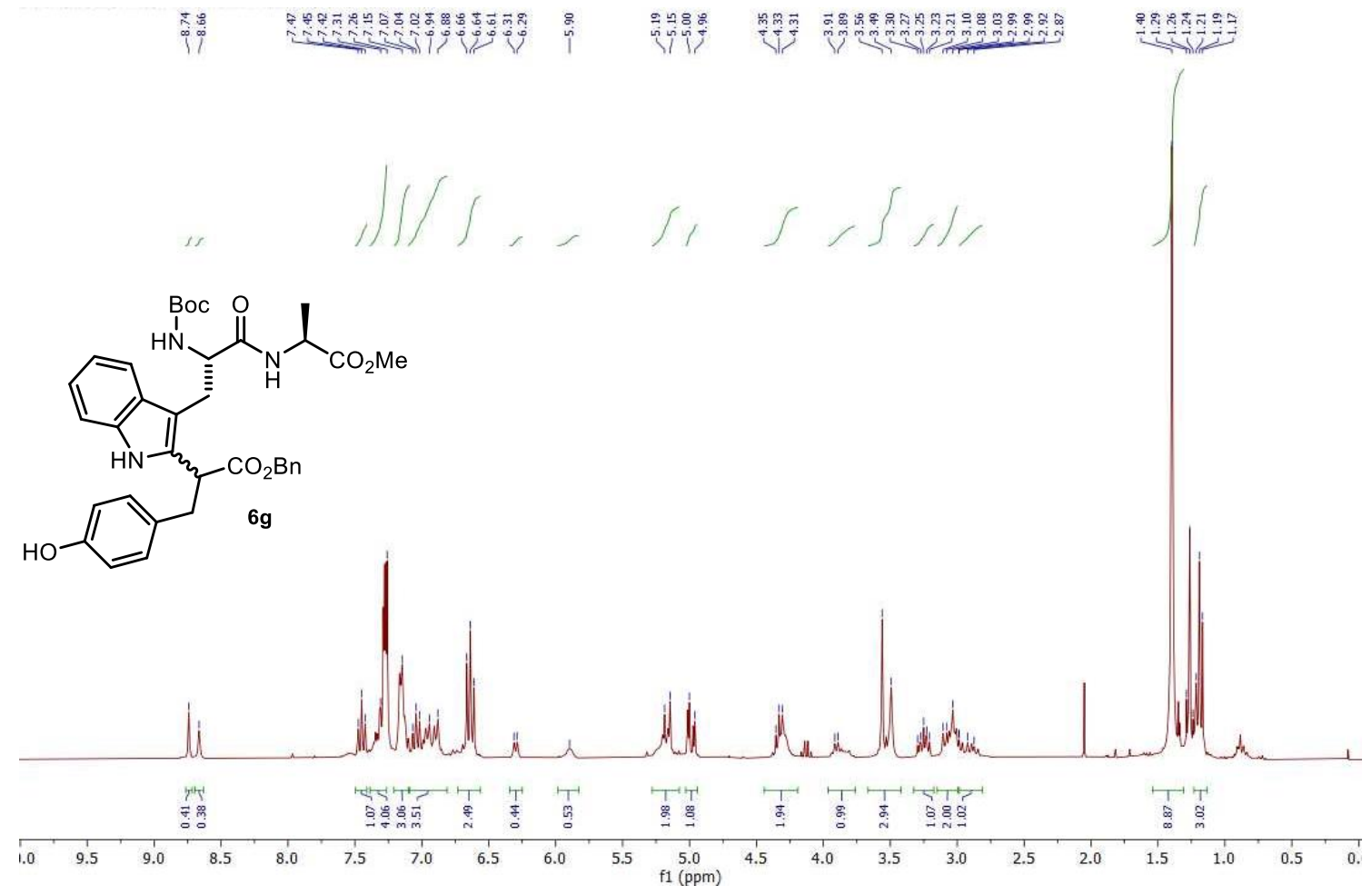

${ }^{13} \mathrm{C}$ NMR of $\mathbf{6 g}\left(126 \mathrm{MHz}, \mathrm{CDCl}_{3}\right)$

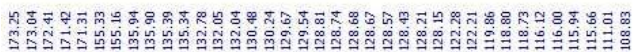

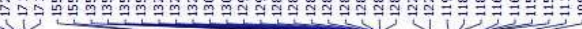

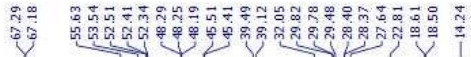

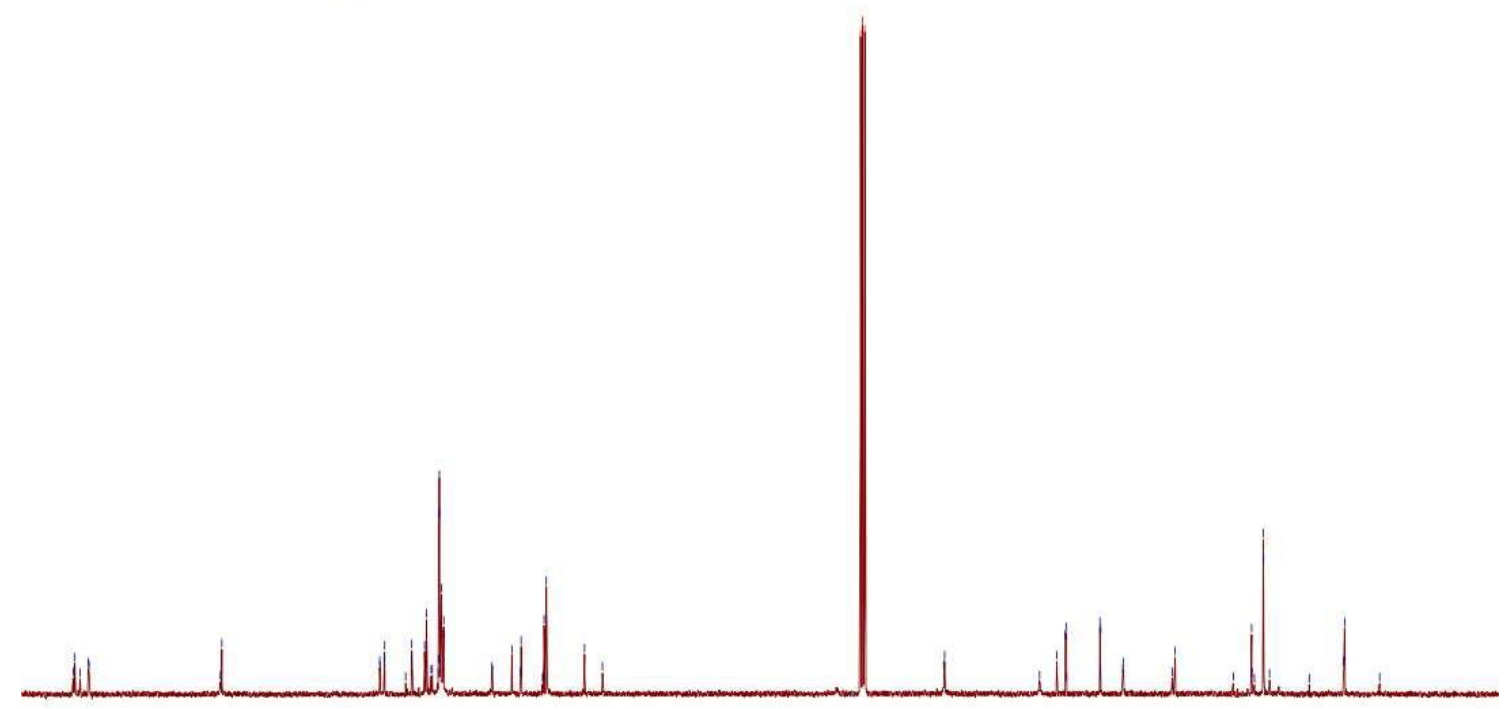

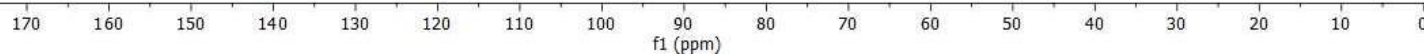


${ }^{1} \mathrm{H} \mathrm{NMR}$ of $\mathbf{6 h}\left(500 \mathrm{MHz}, \mathrm{CDCl}_{3}\right)$

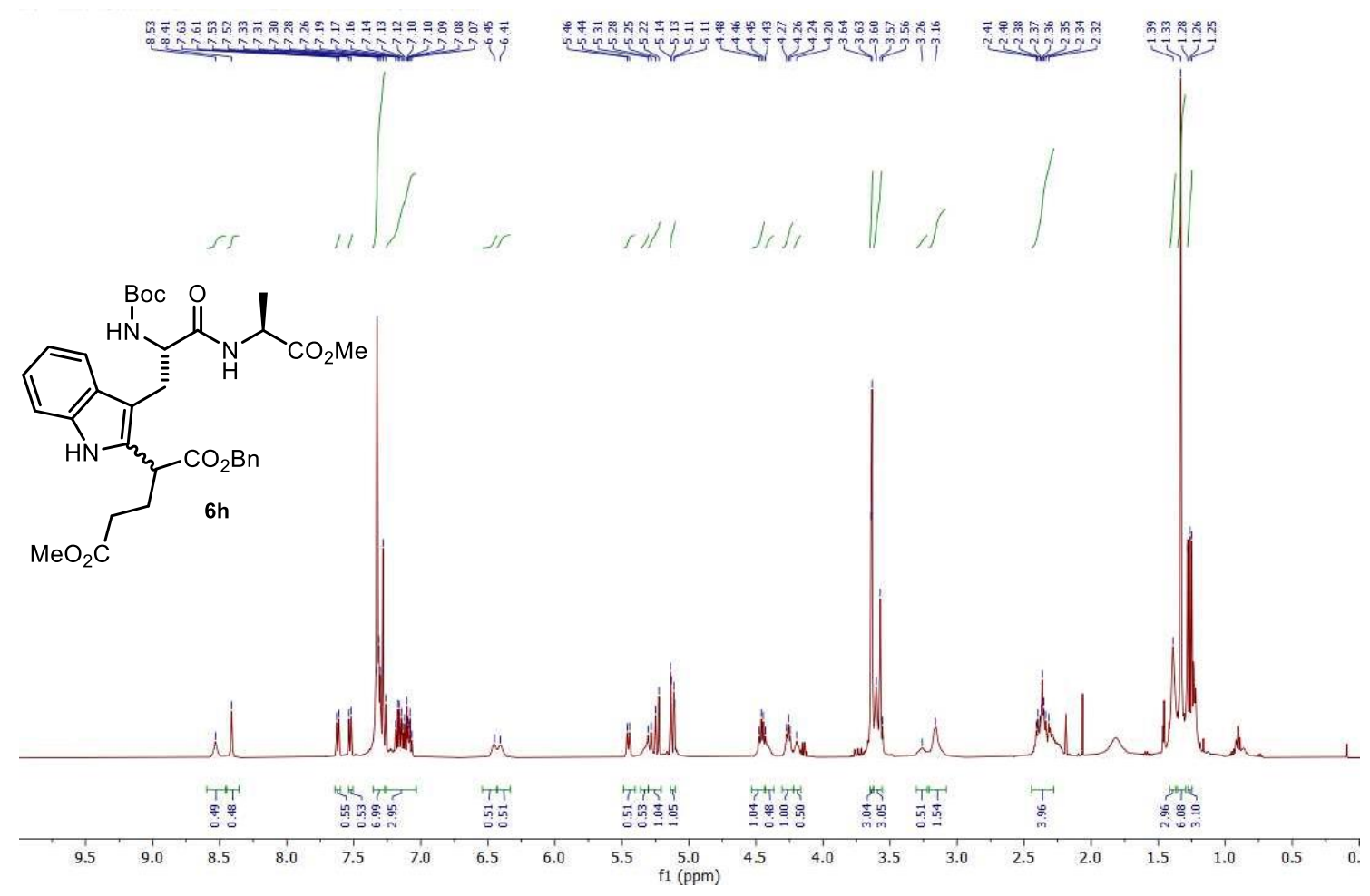

${ }^{13} \mathrm{C}$ NMR of $6 \mathbf{h}\left(126 \mathrm{MHz}, \mathrm{CDCl}_{3}\right)$

GAR-19-13C10.fid

ICIO 13C $\{1 \mathrm{H}\} 512 \mathrm{STBFO} \mathrm{CDCl} 3\{\mathrm{C}: \mathrm{B}$ ruker $T$ TOPSPIN\} gmelchiorre3 19
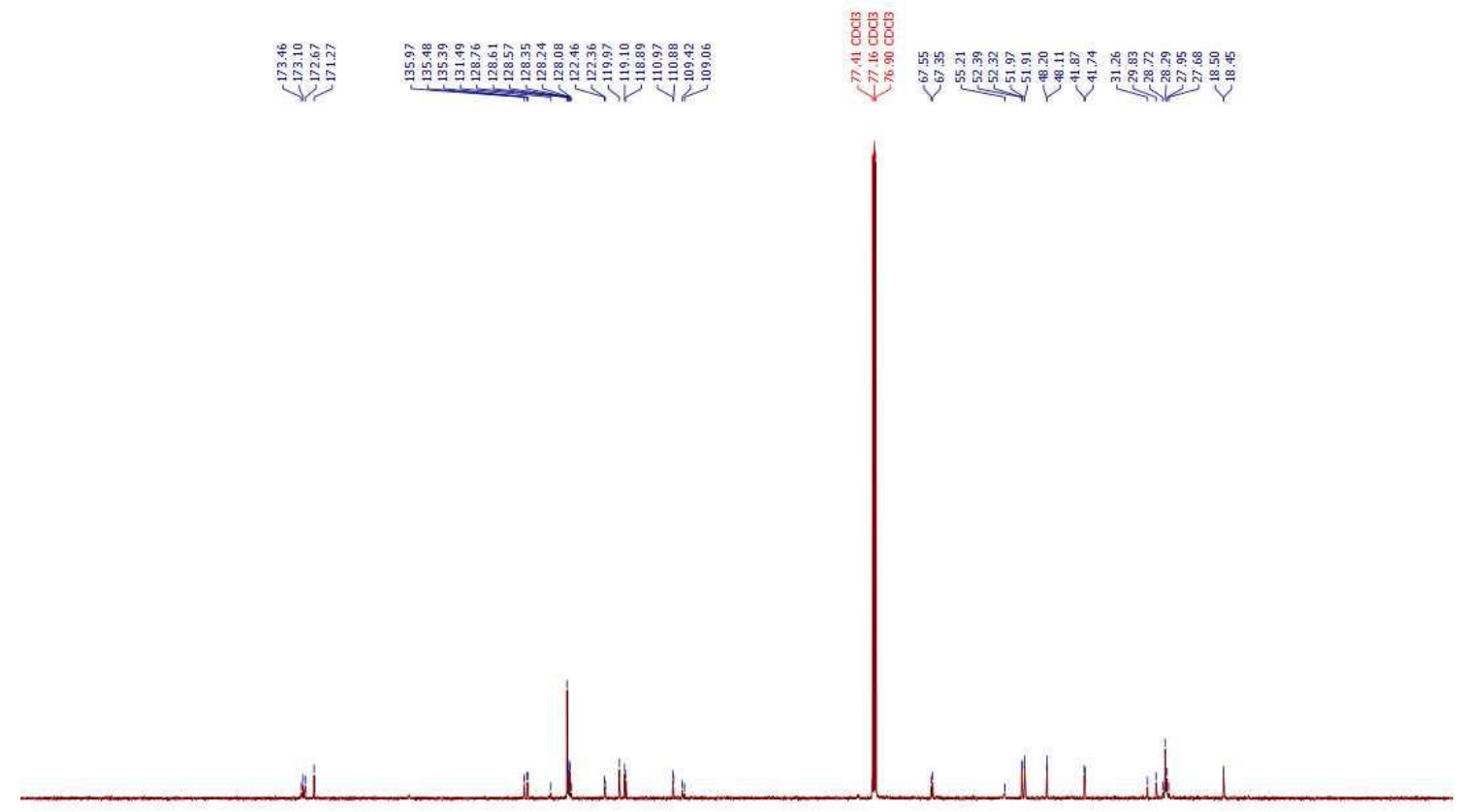

\begin{tabular}{llllllllllllllllllllllllll}
\hline 20 & 210 & 200 & 190 & 180 & 170 & 160 & 150 & 140 & 130 & 120 & 110 & 100 & 90 & 80 & 70 & 60 & 50 & 40 & 30 & 20 & 10 & 0 & -10 & -2
\end{tabular} 
${ }^{1} \mathrm{H}$ NMR of $6 \mathbf{i}\left(400 \mathrm{MHz}, \mathrm{CDCl}_{3}\right.$ )

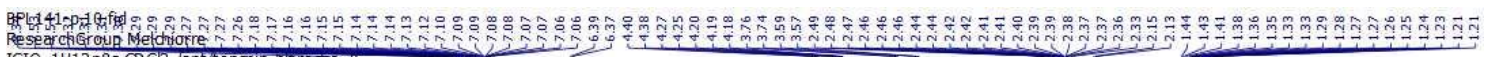

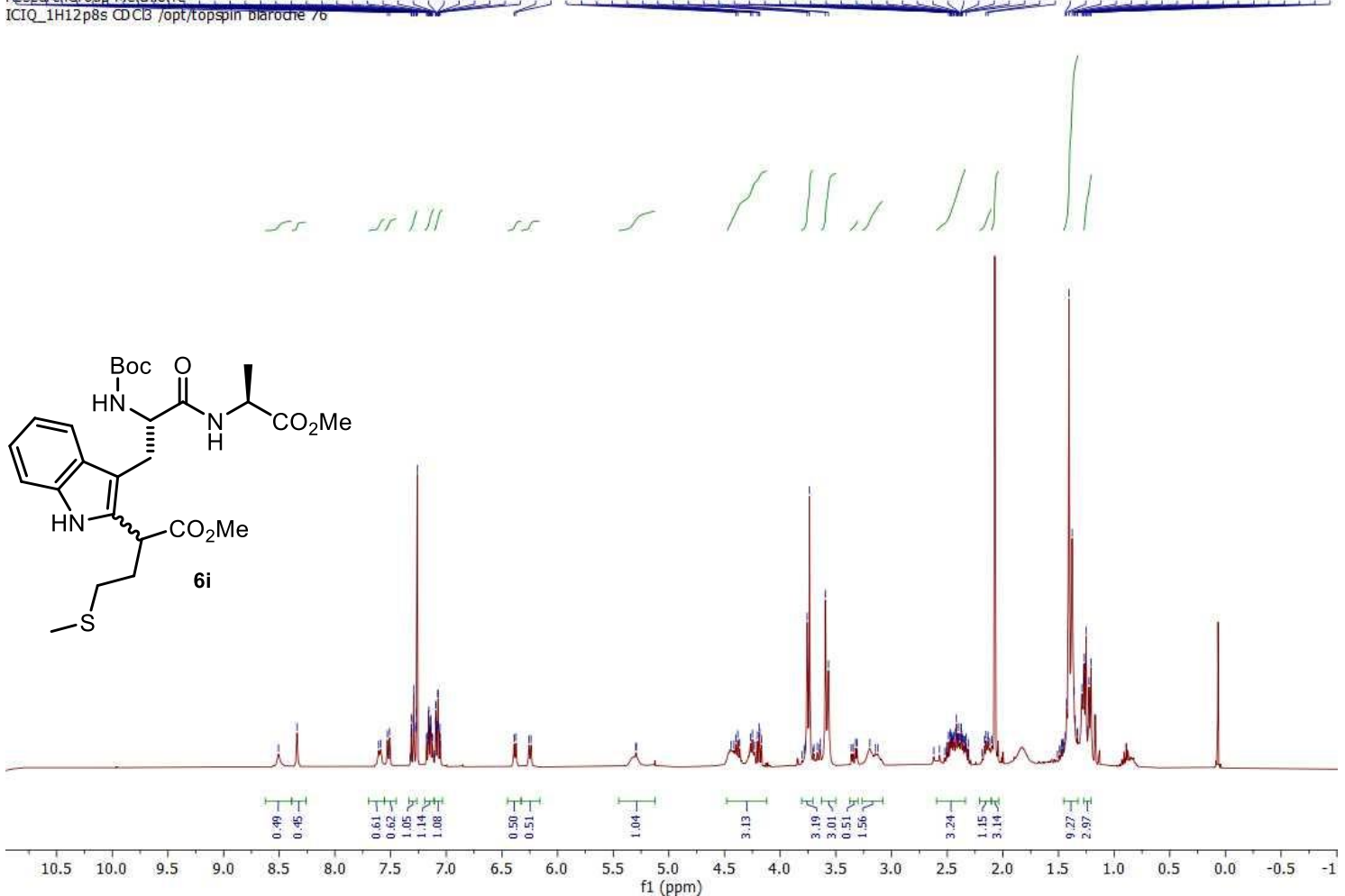

${ }^{13} \mathrm{C}$ NMR of $6 \mathbf{i}\left(126 \mathrm{MHz}, \mathrm{CDCl}_{3}\right)$

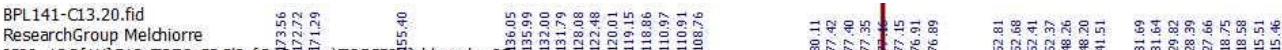

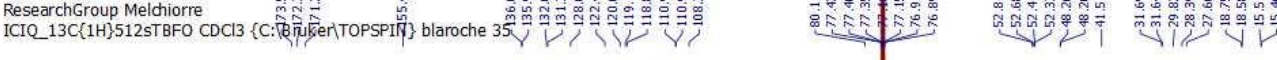

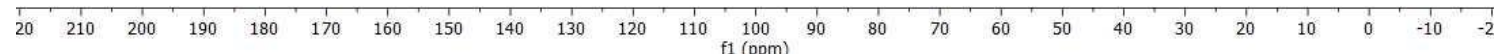


${ }^{1} \mathrm{H} \mathrm{NMR}$ of $\mathbf{6 j}\left(400 \mathrm{MHz}, \mathrm{CDCl}_{3}\right)$

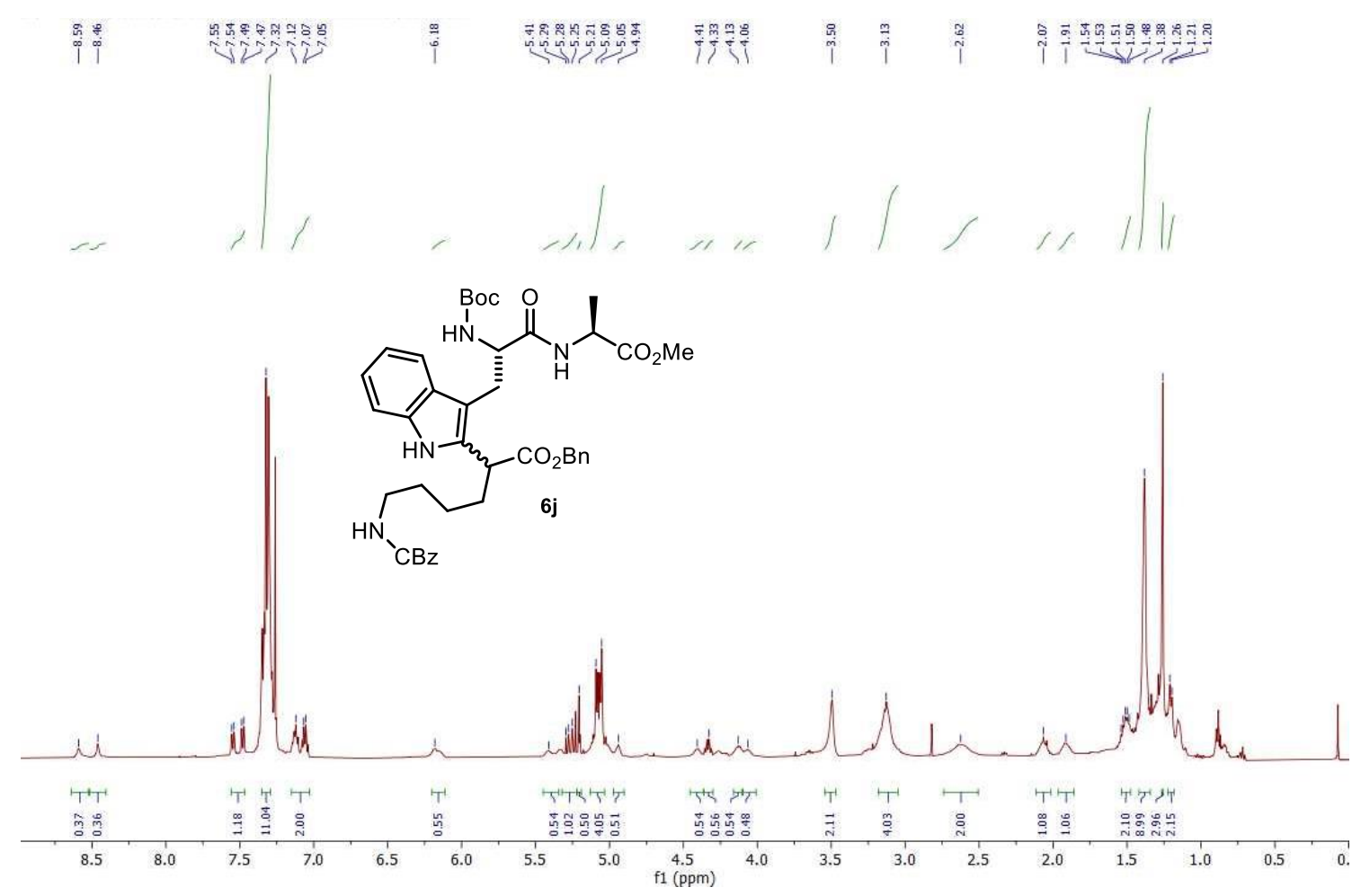

${ }^{13} \mathrm{C}$ NMR of $\mathbf{6 j}\left(126 \mathrm{MHz}, \mathrm{CDCl}_{3}\right)$

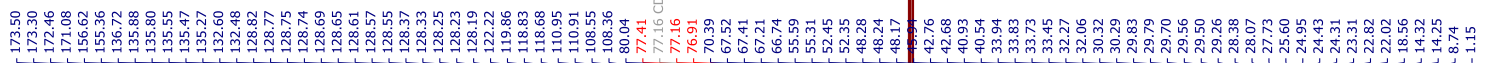

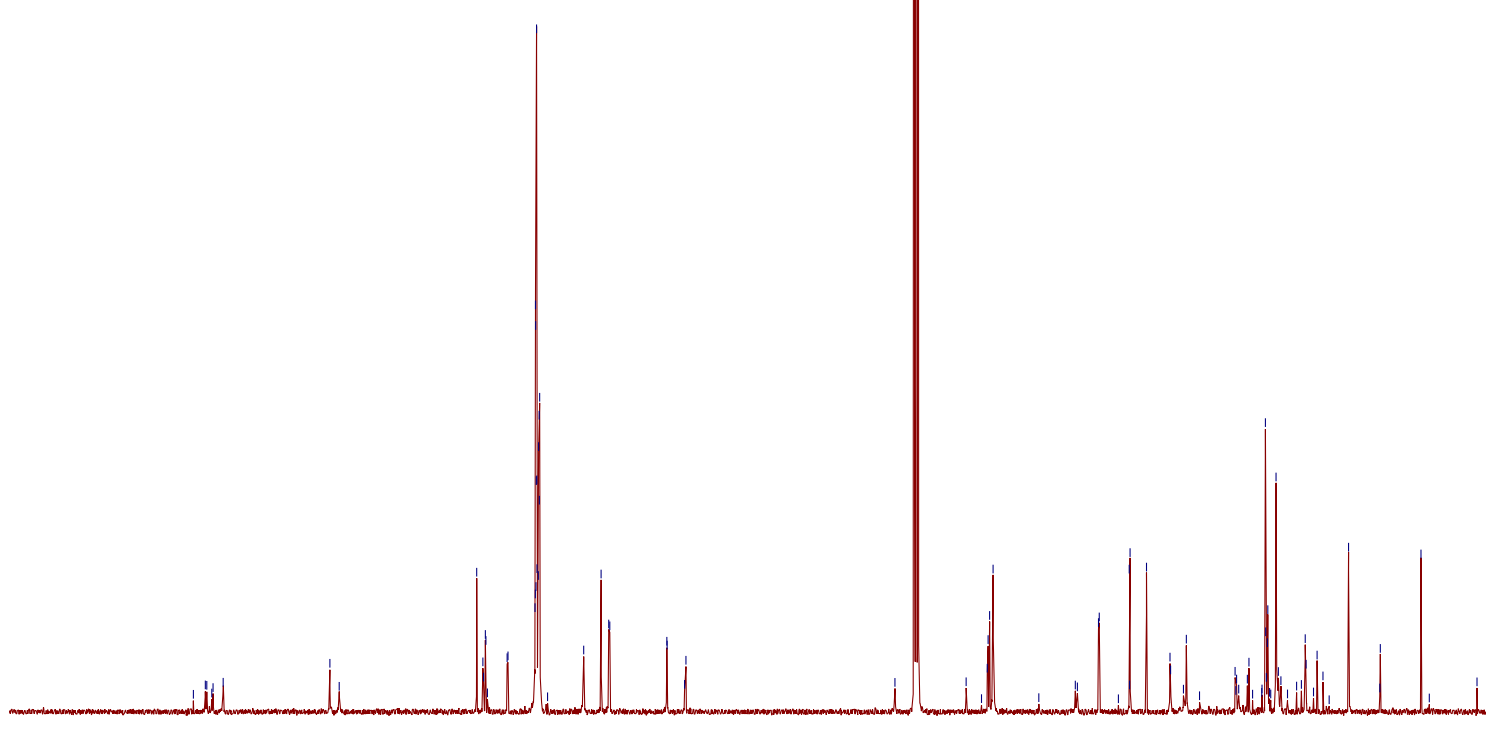

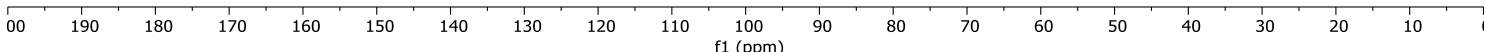

